\title{
Intense Ion Beams for Warm Dense Matter Physics
}

\author{
by \\ Joshua Eugene Coleman \\ Doctotr of Philosophy \\ Department of Engineering-Nuclear Engineering \\ in the \\ Graduate Division \\ University of California, Berkeley \\ and \\ Accelerator Fusion Research Division \\ Ernest Orlando Lawrence Berkeley National Laboratory \\ University of California \\ Berkeley, California 94720
}

Spring 2008

This work was supported by the Director, Office of Science, Office of Fusion Energy Sciences, of the U.S.

Department of Energy under Contract No. DE-AC02-05CH11231. 
Intense Ion Beams for Warm Dense Matter Physics

\author{
by \\ Joshua Eugene Coleman \\ B.S. (University of Akron) 2003 \\ M.S. (University of California, Berkeley) 2005 \\ A dissertation submitted in partial satisfaction \\ of the requirements for the degree of \\ Doctor of Philosophy \\ in \\ Engineering-Nuclear Engineering \\ in the \\ GRADUATE DIVISION \\ of the \\ UNIVERSITY OF CALIFORNIA, BERKELEY \\ Committee in charge: \\ Professor Edward C. Morse, Chair \\ Professor Per F. Peterson \\ Professor Michael A. Lieberman \\ Doctor Peter A. Seidl
}

Spring 2008 
The dissertation of Joshua Eugene Coleman is approved.

\begin{tabular}{lc}
\hline Chair & Date \\
& \\
\hline & Date \\
& \\
\hline & Date \\
\hline & \\
\hline
\end{tabular}

University of California, Berkeley

Spring 2008 
Intense Ion Beams for Warm Dense Matter Physics

Copyright (c) 2008

by

Joshua Eugene Coleman 


\author{
Abstract \\ Intense Ion Beams for Warm Dense Matter Physics \\ by \\ Joshua Eugene Coleman \\ Doctor of Philosophy in Engineering-Nuclear Engineering \\ University of California, Berkeley \\ Professor Edward C. Morse, Chair
}

The Neutralized Drift Compression Experiment (NDCX) at Lawrence Berkeley National Laboratory is exploring the physical limits of compression and focusing of ion beams for heating material to warm dense matter (WDM) and fusion ignition conditions. The NDCX is a beam transport experiment with several components at a scale comparable to an inertial fusion energy driver. The NDCX is an accelerator which consists of a low-emittance ion source, high-current injector, solenoid matching section, induction bunching module, beam neutralization section, and final focusing system. The principal objectives of the experiment are to control the beam envelope, demonstrate effective neutralization of the beam space-charge, control the velocity tilt on the beam, and understand defocusing effects, field imperfections, and limitations on peak intensity such as emittance and aberrations.

Target heating experiments with space-charge dominated ion beams require simultaneous longitudinal bunching and transverse focusing. A four-solenoid lattice is used to tune the beam envelope to the necessary focusing conditions before entering 
the induction bunching module. The induction bunching module provides a head-totail velocity ramp necessary to achieve peak axial compression at the desired focal plane. Downstream of the induction gap a plasma column neutralizes the beam space charge so only emittance limits the focused beam intensity.

We present results of beam transport through a solenoid matching section and simultaneous focusing of a singly charged $\mathrm{K}^{+}$ion bunch at an ion energy of 0.3 $\mathrm{MeV}$. The results include a qualitative comparison of experimental and calculated results after the solenoid matching section, which include time resolved current density, transverse distributions, and phase-space of the beam at different diagnostic planes. Electron cloud and gas measurements in the solenoid lattice and in the vicinity of intercepting diagnostics are also presented. Finally, comparisons of improved experimental and calculated axial focus $(>100 \times$ axial compression, $<2$ ns pulses) and higher peak energy deposition on target are also presented. These achievements demonstrate the capabilities for near term target heating experiments to $\mathrm{T}_{\mathrm{e}} \sim 0.1 \mathrm{eV}$ and for future ion accelerators to heat targets to $\mathrm{T}_{\mathrm{e}}>1 \mathrm{eV}$.

Professor Edward C. Morse

Dissertation Committee Chair 


\section{Contents}

$\begin{array}{ll}\text { Contents } & \text { i }\end{array}$

List of Figures $\quad$ vi

List of Tables $\quad$ xvi

Acknowledgements $\quad$ xvii

1 Introduction 1

1.1 Nuclear Fusion process . . . . . . . . . . . . . . . . . . . . . . . . . 2

1.2 Inertial Fusion Energy (IFE) reactor and power plant concept . . . . 6

1.3 Ion Beam Driven Inertial Fusion . . . . . . . . . . . . . . . . 8

1.4 Warm Dense Matter (WDM) target heating experiments . . . . . . . 9

1.5 The Neutralized Drift Compression Experiment (NDCX) . . . . . . 11

1.5.1 NDCX objectives . . . . . . . . . . . . . . 13

1.5.2 Experimental configurations . . . . . . . . . . . . . . 13

1.5.3 Results reported in this thesis . . . . . . . . . . . . . 17

2 Relevant charged particle physics $\quad 19$

2.1 Introduction . . . . . . . . . . . . . . . . . . . . 19

2.1.1 Coordinate system and units . . . . . . . . . . 19

2.1.2 Plasmas ...................... . . . 20

2.1.3 Charged particle beams . . . . . . . . . . . 20

2.1.4 Single particle motion ............. . . 21 
2.2 Plasma sheaths and parameters . . . . . . . . . . . . . 23

2.2 .1 Debye length . . . . . . . . . . . . . . . . . . . . . . 23

2.2 .2 Plasma frequency . . . . . . . . . . . . . . . . . . 24

2.2 .3 The high-voltage sheath $\ldots \ldots \ldots \ldots \ldots$

2.2 .4 The 1-D Child Law . . . . . . . . . . . . . . . . . 25

2.3 Transverse charged particle beam dynamics $\ldots \ldots \ldots \ldots$

2.3.1 Transverse phase space and emittance . . . . . . . . . 27

2.3.2 Transverse envelope equation . . . . . . . . . . . . . . . 29

2.3.3 Beam transport with solenoids . . . . . . . . . . . . . . . 32

2.3.4 Adverse effects on the transverse beam dynamics . . . . . . 34

2.4 Longitudinal charged particle beam dynamics . . . . . . . . . 37

2.4.1 Longitudinal phase space and emittance . . . . . . . . . 37

2.4.2 Longitudinal envelope equation . . . . . . . . . . . . . 40

2.4.3 Acceleration and bunching methods . . . . . . . . . . . . . 41

2.4.4 Adverse effects on the longitudinal beam dynamics . . . . . . 43

2.5 Coupled longitudinal and transverse beam dynamics . . . . . . . 45

$\begin{array}{lll}3 & \text { Diagnostics, data acquisition and analysis } & 47\end{array}$

3.1 Beam current . . . . . . . . . . . . . . . . . . 47

3.1 .1 Faraday cup . . . . . . . . . . . . . . . . . . 48

$3.1 .2 \quad$ Fast pinhole Faraday cup . . . . . . . . . . . . . . . . 50

3.1 .3 Optical Faraday cup . . . . . . . . . . . . . . . . . 54

3.2 Transverse beam dynamics . . . . . . . . . . . . . . . 58

3.2.1 Beam current density and profile . . . . . . . . . . 58

3.2.2 Transverse phase-space and emittance . . . . . . . . . 61

3.3 Longitudinal beam dynamics . . . . . . . . . . . . . . . . 63

3.3 .1 Longitudinal temperature . . . . . . . . . . . . . 66

3.3.2 Longitudinal phase space . . . . . . . . . . . . . . 67

3.4 Electron cloud and gas measurements . . . . . . . . . . . . . . . . 69

3.4.1 In-bore cylindrical electrodes . . . . . . . . . . . . . . . . 69

3.4 .2 Electrostatic dipoles . . . . . . . . . . . . . . . . 70 
3.5 Pulsed power diagnostics . . . . . . . . . . . . . . . . . 72

3.5 .1 High voltage monitors . . . . . . . . . . . . . . 73

3.5.2 Current monitors . . . . . . . . . . . . . . . . 74

3.6 Data acquisition system $\ldots \ldots \ldots \ldots \ldots$

3.7 Presentation of the data $\ldots \ldots \ldots \ldots \ldots \ldots$

3.7 .1 Oscilloscope signals . . . . . . . . . . . . . . . 76

3.7 .2 Optical images . . . . . . . . . . . . . . . 77

3.7 .3 Transverse beam dynamics . . . . . . . . . . . . . . 78

4 Numerical simulation tools $\quad 82$

4.1 Envelope codes . . . . . . . . . . . . . . . . . . . . . 82

4.1 .1 Java based . . . . . . . . . . . . . . . . . . 83

$4.1 .2 \quad$ MathCAD based . . . . . . . . . . . . . 85

4.1 .3 MATLAB based . . . . . . . . . . . . . . . 87

4.2 Particle-in-cell (PIC) codes . . . . . . . . . . . . . . . . . 89

$4.2 .1 \quad W a r p \ldots \ldots \ldots \ldots \ldots \ldots$

$4.2 .2 \quad$ LSP $\ldots \ldots \ldots \ldots \ldots \ldots \ldots$

5 Beam injection, matching, and transport $\quad 94$

5.1 Ion source and injector . . . . . . . . . . . . . . 94

5.1.1 Beam characterization at the exit of the gun . . . . . . 95

5.2 Pulsed solenoids . . . . . . . . . . . . . . . . . . . . . . 99

5.3 Measurements with two solenoids . . . . . . . . . . . . . 100

5.3.1 Observed electron cloud effects . . . . . . . . . . . . 103

5.3.2 Mitigation of electron cloud effects . . . . . . . . . 106

5.3.3 Alignment effects on beam dynamics . . . . . . . . . . . 108

5.4 Electron cloud studies in four solenoids . . . . . . . . . . . . . . 112

5.4.1 Apertured beam measurements . . . . . . . . . . . . . 113

5.4.2 Apertured beam measurements with electron cloud diagnostics 114

5.4.3 Unapertured beam measurements with electron cloud diagnostics122

5.5 Beam induced gas and electrons . . . . . . . . . . . . . . . 124

5.5 .1 Ionized gas measurement . . . . . . . . . . . . . 126 
5.5.2 Electron emission measurement . . . . . . . . . . . . . . . . 129

5.6 Centroid Motion . . . . . . . . . . . . . . . . . . . . . . . . . 133

5.6.1 Measured centroid offsets . . . . . . . . . . . . . . . 133

5.6 .2 Dipole magnets . . . . . . . . . . . . . . . . . . . 135

5.6 .3 Analytic method . . . . . . . . . . . . . . . . . 137

5.6 .4 Empirical correction . . . . . . . . . . . . . . . . . 139

5.7 Conclusions . . . . . . . . . . . . . . . . . . . . . . . . . 140

6 Beam focusing and bunching 142

6.1 Transverse focusing . . . . . . . . . . . . . . . . . . . . . 143

6.1 .1 NDCX Plasma sources . . . . . . . . . . . . . . . . . . 143

6.1.2 Measurements of neutralized ballistic focusing . . . . . . . 149

6.2 Axial compression . . . . . . . . . . . . . . . . . . . . . 151

6.2.1 Induction Bunching Module (IBM) _ . . . . . . . . . . . 151

6.2 .2 Compression ratio . . . . . . . . . . . . . . . . . . 153

6.2 .3 Compressed pulse width . . . . . . . . . . . . . . . 154

6.2.4 Normalized beam current . . . . . . . . . . . . . . . . 155

6.2.5 Suppressor (middle plate) signal without axial compression . . 155

6.2.6 Suppressor (middle plate) signal with axial compression . . . . 158

6.2.7 Measurements of neutralized axial compression . . . . . . . . 159

6.3 Simultaneous longitudinal bunching and transverse focusing . . . . . 161

6.3.1 Measurements of neutralized ballistic simultaneous focusing . 163

6.4 Chromatic aberrations . . . . . . . . . . . . . . . 166

6.4 .1 Ballistic focusing . . . . . . . . . . . . . 166

6.4 .2 Focusing with a solenoid . . . . . . . . . . . . . . . . 169

6.5 Plasma neutralization challenges with a high field solenoid . . . . . 174

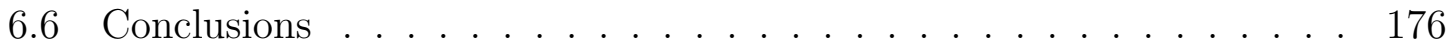

7 Longitudinal beam dynamics measurements 177

7.1 Experimental layout . . . . . . . . . . . . . . . . . . . . 177

7.1.1 Lattice and beam envelopes . . . . . . . . . . . . . 177

7.1 .2 Ion trajectories $\ldots \ldots \ldots \ldots \ldots \ldots \ldots$ 
7.2 Erratum . . . . . . . . . . . . . . . . . . . 182

7.3 Longitudinal temperature measurements . . . . . . . . . . . . . . . 183

7.4 Longitudinal phase space measurements . . . . . . . . . . . . 185

7.4.1 Optical measurements . . . . . . . . . . . . 185

7.4.2 Slit and Faraday collector measurements . . . . . . . . . . 186

7.5 Correlation of the longitudinal measurements with Marx voltage . . . 188

7.6 Conclusions . . . . . . . . . . . . . . . . . . . . . . . 191

8 Conclusions $\quad 193$

8.1 Experimental achievements . . . . . . . . . . . . . . . . 193

8.1.1 Chapter $5 \ldots \ldots \ldots . \ldots \ldots 3$

8.1 .2 Chapter $6 \ldots \ldots \ldots \ldots$

8.1.3 Chapter $7 \ldots \ldots \ldots . \ldots . \ldots . \ldots . \ldots 195$

8.2 Future recommendations . . . . . . . . . . . . . . . . 196

$\begin{array}{ll}\text { Bibliography } & 199\end{array}$ 


\section{List of Figures}

1.1 Average binding energy per nucleon (〈B.E. $\rangle / A)$ for different isotopes. 2

1.2 Deuterium-Tritium fusion reaction. . . . . . . . . . . . . . 3

1.3 Diagram of inertial fusion ignition. . . . . . . . . . . . 6

1.4 Layout of an IFE power plant. . . . . . . . . . . . . . . . . . . . . . 7

1.5 Conceptual design of a hohlraum used for IFE. . . . . . . . . . . . 7

1.6 Ion accelerator components necessary for IFE. . . . . . . . . . . . . 9

1.7 Equation of State diagram for Aluminum displaying different curves for the strong coupling parameter. The region of WDM is marked by a black border. . . . . . . . . . . . . . . . . . 10

1.8 Equation of State diagram for Aluminum displaying different methods of changing solid Al to WDM. The region of WDM is marked by a black border. . . . . . . . . . . . . . . . . . . . .

1.9 Diagram of the Pierce diode geometry and suppression electrodes with removable aperture downstream. . . . . . . . . . . . . . . . 14

1.10 Elevation view of the Two-Solenoid Experiment. . . . . . . . . . . . 15

1.11 Layout of the aperture and suppression electrodes, electron cloud diagnostics: solenoid electrodes (1, 3, 5, and 7), gap electrodes $(2,4$, 6 , and 8); and parallel plate diagnostic relative to the four-solenoid lattice. All the diagnostics have cylindrical symmetry except for the parallel plate diagnostic. . . . . . . . . . . . . . . . . 16

1.12 Elevation view of the Simultaneous Focusing Experiment on NDCX. . 16

1.13 Elevation view of the NDCX with the EEA added on at the exit of the FEPS. . . . . . . . . . . . . . . . 17 
2.1 Pictures of the right hand coordinate system with axes labeled. (a) A view from the left side with the z-axis propagating from left to right and; (b) a view head on with the z-axis coming out of the page. . . .

2.2 Sketch of the cyclotron motion of an ion and electron in a uniform axial magnetic field, $B_{z}$, with a constant axial velocity, $v_{z}$. . . . . . .

2.3 Sketch of the (a) transverse phase space; and (b) the sheared phase space distributions. . . . . . . . . . . . . . . . 27

2.4 Diagram of the Pierce diode geometry and suppression electrodes with removable aperture downstream. . . . . . . . . . . . . .

2.5 (a) Transverse phase space and (b) sheared phase space distributions of a beam with nonlinear focusing effects. . . . . . . . . . . . . .

2.6 Sketch of the (a) Longitudinal phase space; and (b) the sheared distribution with the longitudinal envelope angle ( $\left.z^{\prime}\right)$ removed to show the thermal spread $z_{t h}^{\prime} \ldots \ldots \ldots \ldots \ldots \ldots \ldots \ldots$

2.7 Example of a Marx voltage waveform used to accelerate ions through the diode in Fig. 2.4 . . . . . . . . . . . . . . . . . . . . .

3.1 Diagram of the standard Faraday cup used to measure the beam current at the exit of the diode and different transport configurations on the NDCX. All electrodes and the housing have cylindrical symmetry.

3.2 Photograph of standard Faraday cup used on the NDCX with electrodes labeled. . . . . . . . . . . . . . . . . . . . . . . . .

3.3 Schematic of the capacitive coupling circuit used to monitor the signals on the suppressor and collector electrodes of the Faraday cup. High voltage is applied through a power supply (HV from PS) to the electrode (HV to Electrode). The capacitive coupling allows one to monitor the voltage drop across the resistor (R3). . . . . . . . . .

3.4 Measured beam current on the collector (blue) and image current on the suppressor (red) (a) $28 \mathrm{~cm}$ downstream of the diode $(\mathrm{z}=40 \mathrm{~cm})$ and; (b) $54 \mathrm{~cm}$ downstream of the exit the four-solenoid transport lattice $(\mathrm{z}=304 \mathrm{~cm}) \ldots \ldots \ldots \ldots \ldots \ldots$

3.5 Schematic of the (a) pinhole Faraday cup geometry; (b) photograph of the constructed pinhole Faraday cup and; (c) close-up of the pinhole pattern. . . . . . . . . . . . . . . .

3.6 Measured signal collected by the fast pinhole Faraday cup for a beam (a) with no current density compression and; (b) with current density compression. . . . . . . . . . . . . . . . . . . . . 54

3.7 Schematic layout of the Hamamatsu R1194U series biplanar phototube. 56 
3.8 Measured signal of light collected by the Hamamatsu R1194U phototube for a beam (a) with no current density compression and; (b) with current density compression. . . . . . . . . . . . . . .

3.9 Measurement of the spatial dependence in the integrated light collected by the Hamamatsu R1194U phototube (a) transversely with the LED displaced axially by $10 \mathrm{~cm}$ and; (b) longitudinally with the LED aligned transversely. . . . . . . . . . . . . . . .

3.10 Sketch of the optical diagnostic. . . . . . . . . . . . . 60

3.11 Sketch of the slit-cup diagnostic. . . . . . . . . . . . . . . . . 61

3.12 (a) Sheared phase space distribution integrated over a 500 ns gate in the middle of the $10 \mu \mathrm{s}$ beam pulse; (b) beam envelope as a function of time. . . . . . . . . . . . . . . . . .

3.13 Sheared phase space distribution measured optically over a $500 \mathrm{~ns}$ gate in the middle of the $10 \mu$ s beam pulse. . . . . . . . . . . . 63

3.14 Schematic of the electrostatic energy analyzer used on NDCX. . . . . 64

3.15 (a) Transverse slice of a $305-\mathrm{keV}$ beam integrated over a $500 \mathrm{~ns}$ gate in the middle of a $3 \mu \mathrm{s}$ pulse; (b) intensity of the slice projected onto the energy axis. . . . . . . . . . . . . .

3.16 Longitudinal phase space distribution of the NDCX beam (a) with a 3 $\mu$ s pulse streaked in time and; (b) a $2 \mu$ s pulse measured with two slits. 68

3.17 Layout of the aperture and suppression electrodes, electron cloud diagnostics: solenoid electrodes (1, 3, 5, and 7), gap electrodes $(2,4$, 6 , and 8); and parallel plate diagnostic relative to the four-solenoid lattice. All the diagnostics have cylindrical symmetry except for the parallel plate diagnostic. . . . . . . . . . . . . . . . . 70

3.18 Electron cloud diagnostic signals as a function of time. . . . . . . . 71

3.19 Sketch of the experimental setup using the parallel plates and intercepting diagnostic to measure beam induced gas desorption, ionization, and electron emission. . . . . . . . . . . . . . . . . . 71

3.20 Schematic of (a) a capacitive divider and; (b) a resistive divider used on NDCX. . . . . . . . . . . . . . . . . . 73

3.21 Thresholding of the (a) envelope parameters and; (b) centroid offsets for optical phase space measurements analyzed with Image J. . . . . . 78

3.22 Screen capture of the MATLAB user interface to analyze the transverse phase space. . . . . . . . . . . . . . . . . . . . 79

3.23 Screen capture of the Image $\mathrm{J}$ user interface to analyze the transverse phase space. . . . . . . . . . . . . . . . 
4.1 Example of an input text file with NDCX beam parameters and initial conditions. Units are in meters, radians, and Tesla. . . . . . . . . . 83

4.2 Screen capture of the envelope java code user interface. . . . . . . . . 84

4.3 Calculated (a) transverse envelope and; (b) axial compression ratio downstream of the induction gap. . . . . . . . . . . . .

4.4 Calculated transverse envelopes of different energy slices (a) from the induction gap to the focal plane; (b) near the focal plane and; (c) zoomed in to the see all of the beam waists. Energy slices are color coded in legend. . . . . . . . . . . . . . . . . . . . . .

4.5 Individual Gaussian slices at the focal plane and the composite bunch (dashed blue line). Energy slices are color coded in legend. The left vertical axis is for individual beam bunches and the right vertical axis is for the composite beam bunch. . . . . . . . . . . . . . . . .

4.6 Warp3d simulation of the NDCX diode geometry and suppression electrodes with removable aperture downstream. . . . . . . . . . . . .

4.7 Integrated simulation of a neutralized compressing $300 \mathrm{keV} \mathrm{K}^{+}$ion beam (zoomed into neutralized drift region). The induction gap, energy varaition, and compressing bunch are labeled. . . . . . . . .

5.1 Diagram of the Pierce diode geometry and suppression electrodes with removable aperture downstream (Note aperture not installed for these experiments). . . . . . . . . . . . . . . . . .

5.2 Marx voltage waveform used to extract the beam through the diode (blue) and the measured beam current $28 \mathrm{~cm}$ downstream of the diode $($ red; $z=40 \mathrm{~cm}) \ldots \ldots \ldots \ldots \ldots \ldots \ldots \ldots \ldots \ldots \ldots$

5.3 (a) Sheared phase space distribution integrated over a 500 ns gate in the middle of the $10 \mu \mathrm{s}$ beam pulse; (b) beam envelope as a function of time $15 \mathrm{~cm}$ downstream of the exit of the extractor $(\mathrm{z}=27 \mathrm{~cm}$; no solenoids). . . . . . . . . . . . . . . . . . .

5.4 Transverse profile of the beam measured: (a) $25 \mathrm{~cm}(\mathrm{z}=37 \mathrm{~cm})$ and 31 $\mathrm{cm}(\mathrm{z}=43 \mathrm{~cm})$ downstream of the exit of the extractor. (b) Transverse beam distribution measured at the scintillator plane. All integrated over a 500 ns gate in the middle of the $10 \mu$ s beam pulse (no solenoids). 97

5.5 Transverse beam distribution calculated in Warp at the scintillator plane $(\mathrm{z}=43 \mathrm{~cm})$ : (a) ideally with no emitter gap; (b) with the emitter surface recessed 22 mils back from the knife edge of the Pierce cone. (no focusing with solenoids; note scale differences). . . . . . . . 
5.6 Photographs of a pulsed solenoid throughout the construction stage: (a) wound litz cable ready for first potting; (b) copper cooling water tube was added over the potted coil pack; (c) finished solenoid. . . . .

5.7 Photograph of a three-axis B-dot probe used to map the magnetic fields of the solenoids. . . . . . . . . . . . . . . . . 100

5.8 Axial magnetic field measurements (blue data points) projected onto the $\mathrm{z}$-axis in comparison to a simple, thin-coil model using an on-axis fringe function (red) . . . . . . . . . . . . . . . . . . . . . 101

5.9 Elevation view of the Two-Solenoid Experiment. . . . . . . . . . . 102

5.10 (a) Axial magnetic field profile based on measurements; (b) calculated beam envelope. . . . . . . . . . . . . . . . . . . . . . 102

5.11 (a) Sheared phase space distribution integrated over a $500 \mathrm{~ns}$ gate 1 $\mu \mathrm{s}$ into beam pulse; (b) beam envelope as a function of time $11 \mathrm{~cm} \mathrm{(z}$ $=142 \mathrm{~cm}$ ) downstream of the exit of two solenoids. . . . . . . .

5.12 (a) Signal measured on the suppression electrode at the exit of the second solenoid with a slit paddle intercepting the beam; (b) current calculated for the same case by a Warp simulation (note scale differences).104

5.13 Beam radii: (a) $21 \mathrm{~cm}(\mathrm{z}=152 \mathrm{~cm})$ and; (b) $27 \mathrm{~cm}(\mathrm{z}=158 \mathrm{~cm})$ downstream of the exit of two solenoids as a function of time. . . . . 106

5.14 Sheared phase space distribution integrated over a 500 ns gate in the middle of the $10 \mu$ s beam pulse: (a) measured with a double slit and a Faraday collector; (b) measured optically and; (c) beam envelope as a function of time. All measured $40 \mathrm{~cm}$ downstream of the exit of two solenoids $(\mathrm{z}=171 \mathrm{~cm}) \ldots \ldots \ldots \ldots \ldots \ldots \ldots$

5.15 Transverse profile of the beam measured: (a) $50 \mathrm{~cm}(\mathrm{z}=181 \mathrm{~cm})$ and $56 \mathrm{~cm}(\mathrm{z}=187 \mathrm{~cm})$ downstream of the exit of two solenoids. (b) Transverse beam distribution at the scintillator plane. All integrated over a 500 ns gate in the middle of the $10 \mu$ s beam pulse. . . . . . . . 107

5.16 Transverse beam distribution calculated $56 \mathrm{~cm}$ downstream of the exit of two solenoids at the scintillator plane $(\mathrm{z}=187 \mathrm{~cm})$ using the recessed

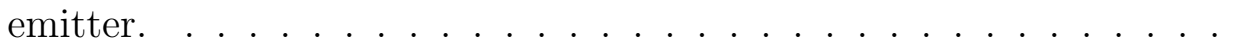

5.17 (a) Transverse beam distribution calculated $56 \mathrm{~cm}$ downstream of the exit of two solenoids at the scintillator plane $(\mathrm{z}=187 \mathrm{~cm})$ using the recessed emitter and misaligned solenoids; (b) beam centroid offsets along the axis of propagation. 
5.18 (a) Transverse beam distribution calculated $56 \mathrm{~cm}$ downstream of the exit of two solenoids at the scintillator plane $(\mathrm{z}=187 \mathrm{~cm})$; (b) Sheared phase space distribution calculated $40 \mathrm{~cm}$ downstream of the exit of two solenoids at the scintillator plane $(\mathrm{z}=171 \mathrm{~cm})$. All calculated using the recessed emitter, misaligned solenoids, and nonlinear focusing terms.111

5.19 Elevation view of the Four-Solenoid Experiment. . . . . . . . . . . . . 112

5.20 (a) Axial magnetic field profile based on measurements; (b) calculated envelope for the apertured 26-mA beam. . . . . . . . . . . . . 113

5.21 Sheared phase space distribution integrated over a 500 ns gate in the middle of the $10 \mu \mathrm{s}$ beam pulse: (a) $15 \mathrm{~cm}$ downstream of the exit of the extractor ( $\mathrm{z}=27 \mathrm{~cm}$; without solenoid focusing); (b) $40 \mathrm{~cm}$ downstream of the exit of four solenoids $(\mathrm{z}=290 \mathrm{~cm}$; note scale differences) and; (c) beam envelope $40 \mathrm{~cm}$ downstream of the exit of four solenoids as a function of time $(\mathrm{z}=290 \mathrm{~cm}) \ldots \ldots \ldots \ldots$

5.22 Layout of the aperture and suppression electrodes, electron cloud diagnostics: solenoid electrodes $(1,3,5$, and 7$)$, gap electrodes $(2,4$, 6 , and 8); and parallel plate diagnostic relative to the four-solenoid lattice. All the diagnostics have cylindrical symmetry except for the parallel plate diagnostic. . . . . . . . . . . . . . . . . . 115

5.23 Electron cloud diagnostic signals as a function of time. . . . . . . . 116

5.24 Charge collected as a function of bias voltage for: (a) electrode 1; (b) electrode 5; (c) electrode 4; (d) electrode 8. . . . . . . . . . . . 117

5.25 Charge collected as a function of bias voltage for electrode 2 . . . . . 118

5.26 Top row: measured transverse beam distribution $56 \mathrm{~cm}$ downstream of the exit of four solenoids $(\mathrm{z}=306 \mathrm{~cm})$; bottom row: measured transverse phase space $40 \mathrm{~cm}$ downstream of the exit of four solenoids ( $\mathrm{z}=290 \mathrm{~cm}$; note scale differences) for: (a) clearing case; (b) grounded case; (c) trapping case. . . . . . . . . . . . . . . . . . . . . . . . . . . 119

5.27 Top row: measured sheared phase space distribution; bottom row: measured envelope as a function of time for: (a) clearing case; (b) grounded case; all $40 \mathrm{~cm}$ downstream of the exit of four solenoids $(\mathrm{z}=$ $290 \mathrm{~cm}) \ldots \ldots \ldots \ldots \ldots \ldots$

5.28 (a) Axial magnetic field profile based on measurements; (b) calculated envelope of $45-\mathrm{mA}$ beam. . . . . . . . . . . . . . . . .

5.29 Comparison of the charge collected on the electron cloud diagnostics for the: (a) apertured 26-mA beam and; (b) 45-mA beam; both for the clearing case (note scale differences). 
5.30 Sheared phase space distribution of the unapertured 45-mA beam integrated over a $500 \mathrm{~ns}$ gate in the middle of the $10 \mu$ s beam pulse: (a) $40 \mathrm{~cm}$ downstream of the exit of two solenoids $(\mathrm{z}=171 \mathrm{~cm})$; (b) 40 $\mathrm{cm}$ downstream of the exit of four solenoids $(\mathrm{z}=290 \mathrm{~cm}$; note scale differences). . . . . . . . . . . . . . . . .

5.31 Sketch of the experimental setup using the parallel plates and intercepting diagnostic to measure beam induced gas desorption, ionization, and electron emission. . . . . . . . . . . . . . . . . . 125

5.32 Beam current signals collected by the Faraday cup (blue), stainless steel plate (red), and copper plate (green) with both parallel plates biased negatively. . . . . . . . . . . . . . .

5.33 (a)Ionized gas current collected on the parallel plates biased at $-10 \mathrm{kV}$ when intercepting the beam with a stainless steel plate (red) and a copper plate (green); (b) ratio of the ionized gas current $\left(\mathrm{I}_{H}\right)$ collected on the negatively biased parallel plates to the beam current $\left(\mathrm{I}_{b}\right)$ versus bias potential over a $200 \mathrm{~ns}$ gate at $3.2 \mu \mathrm{s}$. . . . .

5.34 (a) Secondary electron current collected on the parallel plate biased at $+10 \mathrm{kV}$ when the beam is intercepted with a stainless steel plate (red); and a copper plate (green); (b) Secondary emission coefficient versus

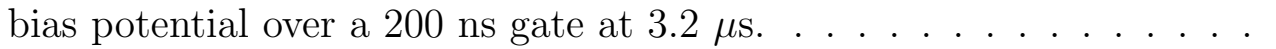

5.35 (a) Measured position offsets from $\mathrm{J}(\mathrm{x}, \mathrm{y})$ measurements for 48 different lattice tunes; (b) measured $\mathrm{J}(\mathrm{x}, \mathrm{y})$ for a subset of eight of these tunes and; (c) measured angular offsets from 4-D phase space measurements. All measured at the exit of the four solenoid lattice. . . . . . . . . . .

5.36 (a) CAD model of a dipole half-shell with the direction of the sheet current (K) shown; (b) a photo of assembled dipole magnet pair with $\mathrm{x}$ and $\mathrm{y}$ dipoles labeled and; (c) a photo of assembled dipole magnet pairs installed on NDCX. . . . . . . . . . . . . . . . . . . . . 136

5.37 Statistical contributions of individual misalignments to the centroid offsets $(\mathrm{a})\langle\mathrm{x}\rangle ;(\mathrm{b})\left\langle\mathrm{x}^{\prime}\right\rangle$ for a lattice tune of 2.6, 1.0, 0.8 and $2.05 \mathrm{~T}$.

6.1 Elevation view of the Simultaneous Focusing Experiment on NDCX. .

6.2 Photograph of (a) a single ferroelectric cylinder; (b) closeup of the stainless steel wires fastened to the aluminum ring and; (c) an individual section of the FEPS with parts labeled.

6.3 Measured (a) voltage waveforms and; (b) current waveforms when operating the 5 sections (shown below) of the FEPS at $9 \mathrm{kV}$. The 5 sections of the FEPS are shown below. . . . . . . . . . . . . . . 146 
6.4 Photograph of an aluminum cathode source closeup (left); inside assembled source (center) with a copper filter coil (right and center). . . 147

6.5 Schematic of FCAPS electrical configuration. . . . . . . . . . 148

6.6 Measured current waveforms when operating the FCAPS at $1 \mathrm{kV}$. . 148

6.7 Neutralized (a) beam radii and; (b) transverse distributions $\mathrm{J}(\mathrm{x}, \mathrm{y})$ at the scintillator plane $(\mathrm{z}=413 \mathrm{~cm})$ versus FEPS time. . . . . . . 149

6.8 Neutralized (a) beam radii and; (b) transverse distributions $\mathrm{J}(\mathrm{x}, \mathrm{y})$ at the scintillator plane $(\mathrm{z}=413 \mathrm{~cm})$ versus FCAPS voltage. . . . . . 150

6.9 Cross section of the IBM used on NDCX. . . . . . . . . . . . 151

6.10 Cartoon of the operation of an induction cell. . . . . . . . . . 152

6.11 (a) IBM waveform comparison to ideal case for 2005 and 2007; (b) improvement in voltage difference (filtered for clarity). . . . . . . . 153

6.12 (a) Measured reduction in ion current signal due to pinholes (red) compared to the signal in a standard Faraday cup; (b) Average scale factor as a function of energy for three consecutive shots. . . . . . . 156

6.13 Schematic of the (a) pinhole Faraday cup geometry; (b) photograph of the constructed pinhole Faraday cup and; (c) close-up of the pinhole pattern. . . . . . . . . . . . . . . 156

6.14 (a) Measured current and (b) charge collected on suppressor (middle plate) without current density compression for a 10- $\mu$ s beam pulse. . 157

6.15 (a) Measured current; (b) zoomed in to view capacitive image current and; (c) charge collected on suppressor (middle plate) with current density compression for $3-\mu$ s beam pulse. . . . . . . . . . . . .

6.16 Energy dependence at the axial focal plane shown for (a) single shots and; (b) multiple shots at different beam energies. . . . . . . . . 160

6.17 (a) Current waveform at the focal plane for a neutralized $\mathrm{K}^{+}$ion beam measured with the fast Faraday cup (FFC) (blue) and phototube (PT) (green) and; (b) pulse widths measured for multiple shots near the axial focal plane. . . . . . . . . . . . . . . . . 161

6.18 (a) Focusing lattice used to control: (b) the ideal beam envelope. . . 162

6.19 Time dependent transverse beam distributions demonstrating the simultaneous focal plane. . . . . . . . . . . . . . . . 164

6.20 Projected spot radii (2rms) for distributions in Fig. 6.19 . . . . . . 164 
6.21 Comparison of experimental measurements on NDCX (blue) versus calculations made with LSP (maroon and red) at the focal plane: (a) compressed current profile with a 1-ns Gaussian response resolved from the raw LSP solution (red) and; (b) line-out of the transverse distribution shown in Fig. 6.19. . . . . . . . . . . . . . . . . .

6.22 Calculated (a) change in beam energy and; (b) transverse envelope angle due to the velocity tilt. . . . . . . . . . . . . .

6.23 Calculated transverse envelopes of different energy slices (a) from the induction gap to the focal plane and; (b) near the focal plane. Energy slices are color coded in legend. . . . . . . . . . . . . . . . . . 168

6.24 Individual Gaussian slices at the focal plane and the composite bunch (dashed blue line). Energy slices are color coded in legend. The left vertical axis is for individual beam bunches and the right vertical axis is for the composite beam bunch. . . . . . . . . . . . . .

6.25 Measured plasma density and expected beam density for a perfectly neutralized case both near the target. . . . . . . . . . . . . . . . 170

6.26 Elevation view of the Final Focusing Experiment on NDCX with changes from Fig. 6.1 labeled in red. . . . . . . . . . . . . . . . . 170

6.27 Calculated (a) change in axial velocity and; (b) transverse envelope angle of different energy slices. . . . . . . . . . . . . . .

6.28 Calculated transverse envelopes of different energy slices (a) from the induction gap to the focal plane; (b) near the focal plane and; (c) zoomed in to the see all of the beam waists. Energy slices are color coded in legend. . . . . . . . . . . . . . . . . .

6.29 Individual Gaussian slices at the focal plane and the composite bunch (dashed blue line). Energy slices are color coded in legend. The left vertical axis is for individual beam bunches and the right vertical axis is for the composite beam bunch. . . . . . . . . . . . . . .

6.30 Measured plasma density and expected beam density for a perfectly neutralized case both near the target. . . . . . . . . . . . . 174

6.31 Magnetic flux calculations in the final focus geometry (a) with the solenoid only; (b) including eddy current effects from the stainless steel beam pipe and flanges. Note the geometries are slighly different in both cases. . . . . . . . . . . . . . . . . .

7.1 Elevation view of the NDCX with the EEA added on at the exit of the FEPS. . . . . . . . . . . . . . . . 178 
7.2 (a) Axial magnetic field profile based on measurements; (b) calculated beam envelope for an unneutralized beam and; (c) calculated beam envelope for a beam neutralized by the FEPS. . . . . . . . . . . .

7.3 Schematic of the ion trajectories though the spectrometer. Ions with an energy greater than the dipole $\left(\mathrm{E}_{3}\right.$, blue) are shown with a larger radius of curvature and ions with an energy less than the dipole $\left(\mathrm{E}_{1}\right.$, red) are shown with a smaller radius of curvature. The coordinate system is shown at the entrance and exit of the spectrometer. . . . .

7.4 Displacement of energy profiles transported through the spectrometer about the focal plane. All profiles are transverse slices of the beam integrated over a $500 \mathrm{~ns}$ gate in the middle of the $3 \mu$ s pulse projected onto the $\mathrm{x}$-axis at the focal plane. The equivalent energy on the dipole is labeled. . . . . . . . . . . . . . . . . . .

7.5 Displacement of energy profiles transported through the spectrometer about the focal plane. All profiles are transverse slices of the beam integrated over a $500 \mathrm{~ns}$ gate in the middle of the $3 \mu$ s pulse projected onto the $\mathrm{x}$-axis at the focal plane. The corrected equivalent energy on the dipole is labeled. . . . . . . . . . . . . . . . . 183

7.6 Top row: Transverse slices of the beam integrated over a 500 ns gate in the middle of a $3 \mu$ s pulse. Bottom row: Intensity of the transverse slices projected onto the energy axis for: (a) an unneutralized beam and; (b) a beam neutralized by the FEPS (note scale differences). . .

7.7 Longitudinal phase space distribution of the NDCX beam with a $3 \mu \mathrm{s}$ pulse streaked in time. . . . . . . . . . . . . 186

7.8 Longitudinal phase space distribution of the NDCX beam with a $2 \mu \mathrm{s}$ pulse measured with two slits. . . . . . . . . . . . . 187

7.9 Correlation of the Marx voltage with individual measurements of the energy profile made with the CCD camera (note scale differences). . . 189

7.10 Correlation of the Marx voltage (blue) with the $\langle\mathrm{E}\rangle$ (red) resolved from longitudinal phase space measurements with: (a) the streak camera for a $3 \mu$ s pulse and; (b) the slit-cup for a $2 \mu$ s pulse (note scale differences). 190

7.11 Longitudinal phase space distributions calculated in: (a) LSP and; (b) Warp. . . . . . . . . . . . . . . . . . . . . . 191 


\section{List of Tables}

1.1 Comparison of beam parameters for the front end of an Inertial Fusion Energy driver and the NDCX. . . . . . . . . . . . . . .

1.2 Comparison of beam parameters for NDCX and a future WDM accelerator. . . . . . . . . . . . . . . . . .

3.1 Parameters for the electrostatic energy analyzer used on the NDCX. .

3.2 Details of different current pulsers on NDCX and the specification of the current transformers used to monitor the current signals. . . . . .

5.1 Approximation of $\mathrm{x}$ and $\mathrm{y}$ displacements of the two solenoids relative to the ideal beam axis. . . . . . . . . . . . . . . . . . 110

5.2 Comparison of the charge collected on the electron cloud diagnostics for the $45-\mathrm{mA}$ beam and the apertured $26-\mathrm{mA}$ beam for the clearing case. . . . . . . . . . . . . . . .

5.3 Calculated ionized gas densities immediately after the beam head (3.2 - $3.4 \mu \mathrm{s})$ and at the end of the beam pulse when intercepting the beam with stainless steel and copper plates. . . . . . . . . . . . . .

5.4 Calculated secondary emission coefficient and electron densities immediately after the beam head $(3.2-3.4 \mu \mathrm{s})$ and at the end of the beam pulse when intercepting the beam with stainless steel and copper plates. 132

5.5 Measured centroid correction at the exit of the four-solenoid lattice using the last two pairs of dipoles. . . . . . . . . . . . . . . 139 


\section{Acknowledgements}

The work that I am reporting in this thesis was supported by the Director, Office of Science, Office of Fusion Energy Sciences, of the U.S. Department of Energy under Contract No. DE-AC02-05H11231 and DE-AC02-76CH3073. It also would not have been possible without the continued support of the Heavy Ion Fusion Sciences Virtual National Laboratory in the Accelerator and Fusion Research Division at Lawrence Berkeley National Laboratory (LBNL).

I would like to specially thank all of the technical support staff from Building 58 who maintained the NDCX facility. Mechanical and vacuum technicians include Tak Katayanagi, Eugene Flor, Michael Dickinson, Cory Lee, and Wayne Greenway. Tak Katayanagi did an excellent job of promptly maintaining the facility to meet programmatic deadlines. He also did a pretty good job of making the work a little more enjoyable with his sense of humor. Electrical technicians include Craig Rogers, Ed Romero, and Mark Regis. Building managers Wayne Greenway and Bill Gath did an excellent job of supervising the work taking place on the NDCX facility.

I would like to thank Matthaeus Leitner and Jin-Young Jung for their mechanical design efforts and drawings incorporated in this thesis. I would also like to thank Will Waldron for his continued efforts in supervising electrical maintenance, electrical design and providing electrical schematics used in this thesis.

I would like to thank Dale Welch, Irving Haber, Steve Lund, and John Barnard for their useful discussions and assistance with theory and computational work. We would not have good agreement without you guys.

I would like to thank Doug Ogata and Chris Wooton (in memoriam) for their invaluable undergraduate assistance. Much of their calculations and data analysis are incorporated in this thesis. 
I would like to thank my thesis advisor Ed Morse and my supervisor Peter Seidl at LBNL for providing useful advice and motivation throughout my studies.

Finally I would like to thank all of my friends and family, you know who are. Hanging out with you guys always makes life worth the challenges. Especially my best friend Christie Park nothing lights up your day like a day with Christie. 


\section{Chapter 1}

\section{Introduction}

The energy demands on earth are steadily increasing and will continue to do so due to both population growth and the industrialization of developing countries. Consequently, global fossil fuel reserves are diminishing as greenhouse gas emissions increase. As the world suffers both economically and enviornmentally from the inefficiencies of fossil fuels, the need for cheaper and more effective energy alternatives is imminent. While renewable energy sources like solar, wind, and hydroelectric are costly and fail to yield high amounts of energy, exploring the various methods of achieving nuclear power illustrate its clear advantages.

Nuclear power offers solutions which contain at least five orders of magnitude higher energy density than conventional fossil fuels. There are two processes by which energy can be released from nuclear reactions. The average binding energy per nucleon $(\langle$ B.E. $\rangle / \mathrm{A})$ of the isotope depicts whether the isotope can be used for a nuclear fission reaction (splitting heavy atoms) or nuclear fusion reaction (combining light atoms) (Fig. 1.1). The fuel for these processes is readily abundant and there are no emissions. However, all of the current nuclear reactors on the planet produce energy through fission processes, producing alarming amounts of radioactive waste. 


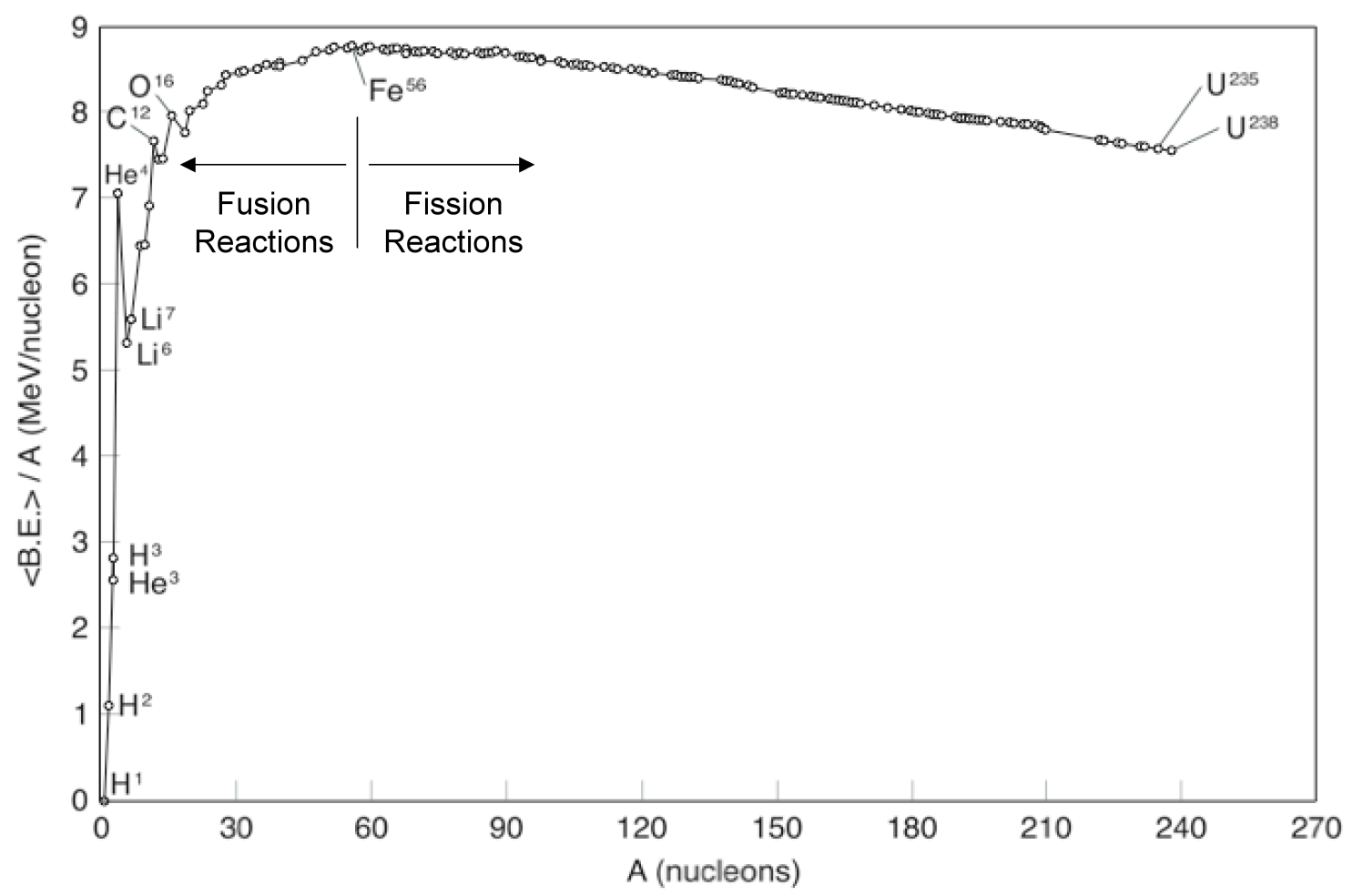

Figure 1.1. Average binding energy per nucleon (〈B.E. $\rangle / A)$ for different isotopes.

This waste presents storage and proliferation issues. All of the problems associated with fission confirm nuclear fusion as a more viable alternative for energy production.

\subsection{Nuclear Fusion process}

Nuclear fusion is the fundamental energy source of the sun and other stars. It is the process of combining light nuclei to yield heavier products, which release energy. In order for a fusion reaction to take place the reactants must overcome the repulsive Coulomb force and possess a high enough cross section. The cross section (or probability) of the reaction is a function of the temperature or thermal energy of 


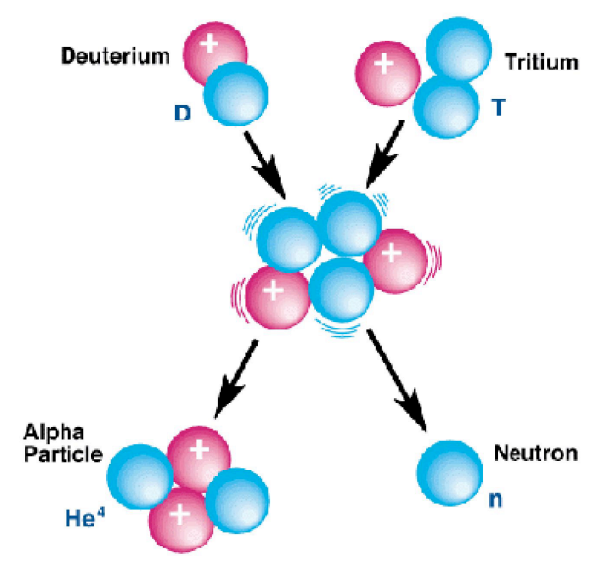

Figure 1.2. Deuterium-Tritium fusion reaction.

the reactants. The deuterium and tritium fusion reaction (Fig. 1.2) has the highest cross section $\left(\sim 5 \times 10^{-28} \mathrm{~m}^{2}\right)$ achievable for the lowest temperature $(\sim 150 \mathrm{keV})$ of any fusion reaction. However, this temperature still remains near $10^{9} \mathrm{~K}$, and is only achieved with a plasma. The amount of energy released from this reaction is 17.6 $\mathrm{MeV}$, a $3.5 \mathrm{MeV}$ alpha particle and a $14.1 \mathrm{MeV}$ neutron (Eq. 1.1).

$$
D+T \rightarrow^{4} H(3.5 \mathrm{MeV})+n(14.1 \mathrm{MeV})
$$

The neutron has such a high energy and low cross section that it does not react with any of the remaining fuel. The neutron does however help provide the energy for electricity production through nuclear stopping, heat, and energy transfer processes. Neutrons are also necessary for the process of breeding tritium fuel, which does not exist naturally on earth. The alpha particle on the other hand does help heat the fuel and sustains additional fusion reactions.

The Lawson criterion, developed by by John D. Lawson in 1955 [1] and published in 1957 [2] is the break-even point in a fusion reactor, or ignition, where the products of the fusion reactions can heat the remaining plasma enough to self-sustain further 
reactions. As originally formulated the Lawson criterion gives a minimum required value for the product of the plasma electron density $n_{e}$ and the energy confinement time $\tau_{E}$.

The energy confinement time, $\tau_{E}$, measures the rate at which a system loses energy to its environment. It is the fusion plasma energy density $U_{t h}$ divided by the power loss per unit volume $P_{\text {loss }}$ :

$$
\tau_{E}=\frac{U_{t h}}{P_{l o s s}} .
$$

The average Maxwellian plasma energy density $U_{t h}$ is defined below.

$$
U_{t h}=\frac{3}{2}\left(n_{e} T_{e}+n_{i} T_{i}\right)=3 n_{e} T_{e}
$$

The equation on the right is yielded assuming electron and ion densities $\left(n_{e}, n_{i}\right)$ and temperatures $\left(T_{e}, T_{i}\right)$ are equal and there are no impurities in the plasma. The power density yielded from a D-T fusion plasma assuming equal mixture of deuterium to tritium is shown below.

$$
P_{f}=n_{D} n_{T}\langle\sigma v\rangle E_{f}=\frac{n_{e}^{2}}{4}\langle\sigma v\rangle E_{f}
$$

where $n_{D}$ and $n_{T}$ are the deuterium and tritium ion densities, $\langle\sigma v\rangle$ is the reaction rate which is the product of the cross section of the reaction and average Maxwellian velocity, and $E_{f}$ is the amount of energy released from the D-T fusion reaction (17.6 $\mathrm{MeV})$.

Assuming an ideal D-T fusion plasma with no impurities and perfect energy transfer the only source of energy loss is through alpha particle heating $\left(E_{\alpha}\right)$. This accounts for $\sim 1 / 5$ of the energy from the $\mathrm{D}-\mathrm{T}$ fusion reaction or $3.5 \mathrm{MeV}$. The power loss per unit volume, $P_{\text {loss }}$, can be defined by substituting $E_{\alpha}$ for $E_{f}$ in Eq. (1.4). Substituting 
this and $U_{t h}$ from Eq. (1.3) into Eq. (1.2) the Lawson criterion is now defined:

$$
n_{e} \tau_{E}=\frac{3 n_{e} T_{e}}{\frac{n_{e}}{4}\langle\sigma v\rangle E_{\alpha}}=\frac{12 T_{e}}{\langle\sigma v\rangle E_{\alpha}} .
$$

There are two approaches to achieving fusion energy. The first approach, Magnetic Fusion Energy (MFE), dates back as far as 1946 and five years later the U.S. MFE program began in 1951. In a MFE reactor, a burning plasma is confined by its own magnetic fields, created by the plasma's current flow, and strong magnetic fields provided by external magnetic field coils [4].

The second approach Inertial Fusion Energy was first suggested in 1962 by scientists at Lawrence Livermore National Laboratory. In an IFE reactor a fusion capsule is imploded by a high powered driver [4].

Both methods have a different approach to satisfy the Lawson criterion. In a MFE reactor, the plasma densities peak around $10^{20} \mathrm{~m}^{3}$ forcing the plasma confinement times to be greater than several seconds. For IFE, the reactions take place in $\sim 10^{-9}$ s but the densities will approach $10^{30} \mathrm{~m}^{3}$.

In IFE a small pellet $(\mathrm{r} \sim 1 \mathrm{~mm})$ of DT fuel $(\mathrm{m} \sim 10 \mathrm{mg})$ is rapidly heated by some driving force of radiation (light, x-rays, ions or electrons) (Fig. 1.3). The fuel is then compressed to $10^{3}$ times its liquid density. This compression ignites the fuel at about 5-10 keV causing the fusion reactions to take place. The alpha particles produced in the reaction heat up the surrounding fuel and thermonuclear burn spreads throughout the compressed fuel.

Fusion power can be generated for commercial electricity production by igniting pellets several times per second. The National Ignition Facility (NIF), the largest laser facility in the world, has already begun target heating experiments and is expected to begin experiments with all of its 192 lasers next year (2009). One of NIF's objectives is to demonstrate the proof of principle of a self sustaining inertial fusion reaction [3]. 


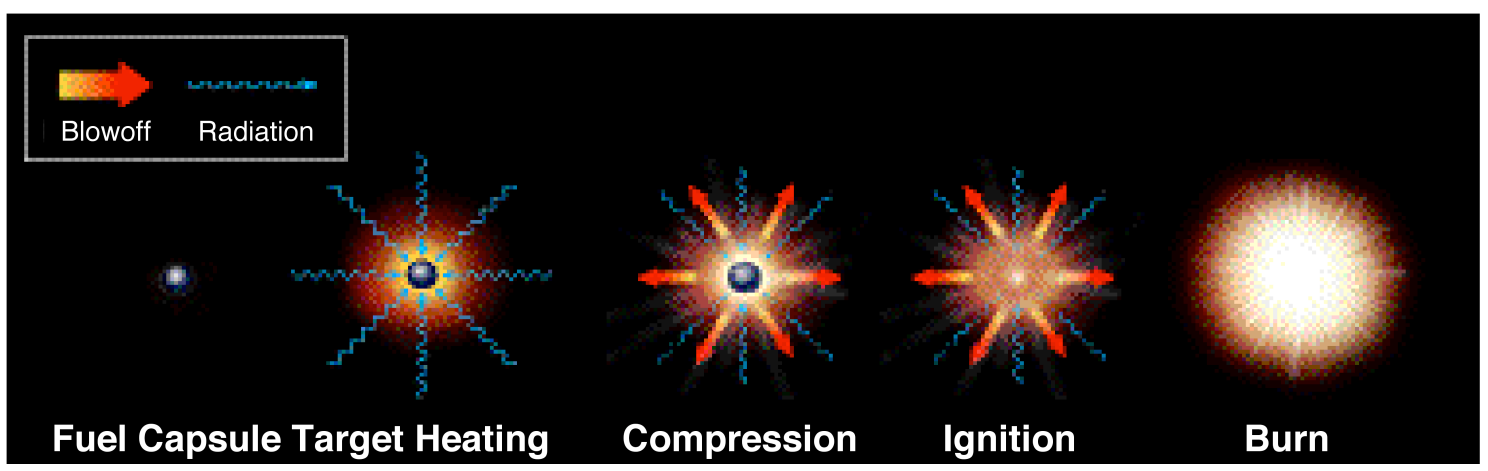

Figure 1.3. Diagram of inertial fusion ignition.

\subsection{Inertial Fusion Energy (IFE) reactor and power plant concept}

An inertial fusion power plant consists of several components (Fig. 1.4). A driver with MJ of energy is necessary to heat and compress the target for fusion ignition. The driver requires a pulse length less than the hydrodynamic disassembly time of the target $(\sim 10 \mathrm{~ns})$ and must deposit all of its energy in a small spot $(\sim 1 \mathrm{~mm})$.

A reactor chamber that can efficiently recover the fusion energy released and sustain minimal radiation damage is essential. Lastly, a target factory must also be on site to provide an ample supply of targets because the desired repetition rate of implosions is on the order of $10 \mathrm{~Hz}$. After the fusion energy is converted into thermal energy a steam plant will be used to convert the thermal energy into electricity.

Inertial fusion reactions can be driven directly or indirectly. Methods of direct drive have the source of radiation, typically lasers or ion beams, focused directly on the fusion target. The advantage of this driver scenario is the higher coupling efficiency with potential for higher gains.

Indirect drive methods use a cylindrical can, or holraum (Fig. 1.5), to convert 


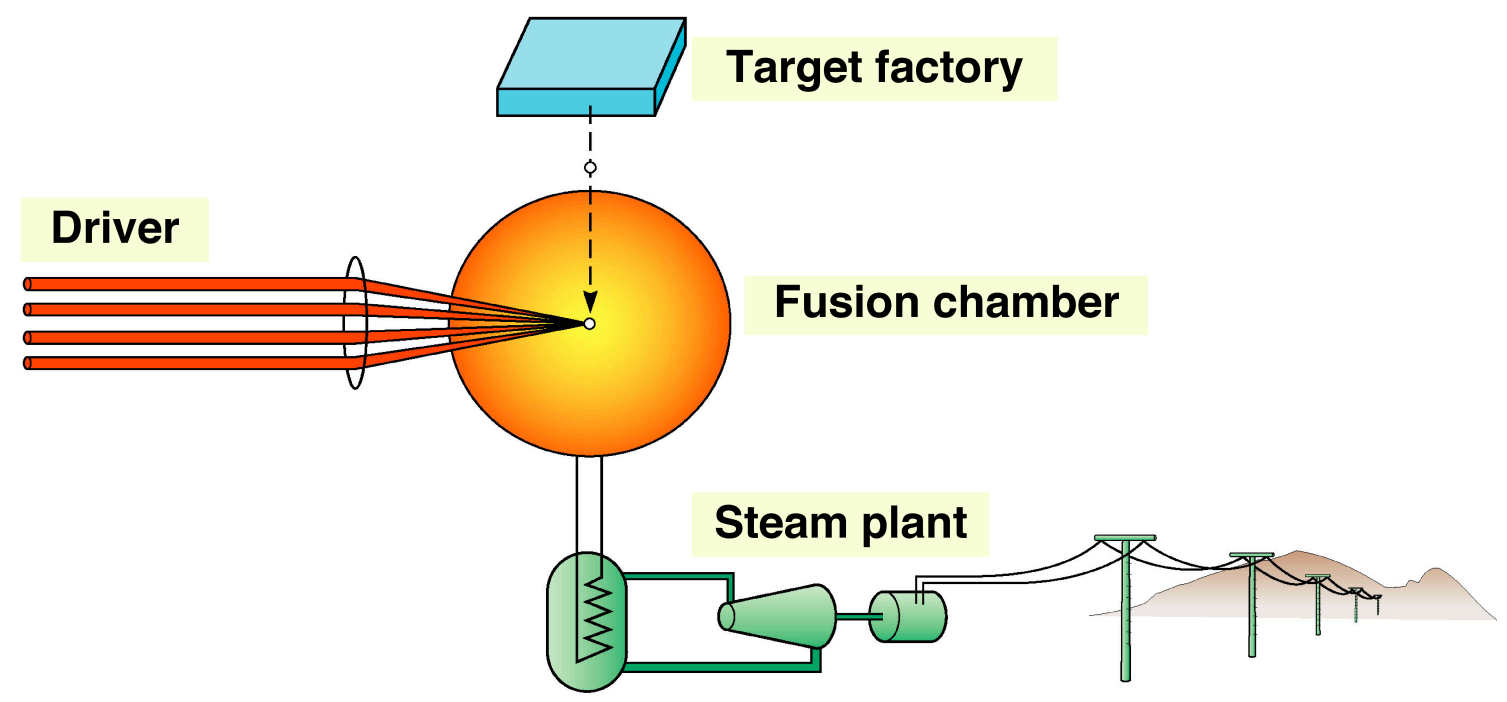

Figure 1.4. Layout of an IFE power plant.

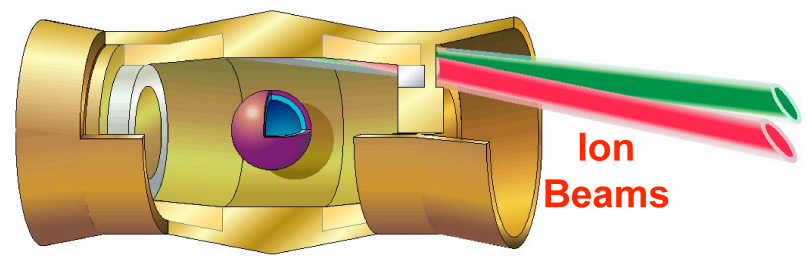

Figure 1.5. Conceptual design of a hohlraum used for IFE.

the driver's energy into a uniform field of x-ray radiation. The advantage of indirect drive is that it relaxes the requirement of beam uniformity necessary for direct drive. Also, the absorption of thermal x-rays by the target is more efficient than the direct absorption of laser light, however hohlraums take considerable energy to convert the driver energy to x-rays which in turn reduces the overall efficiency of laser-to-target energy transfer. Thus, the debate regarding the superior efficiency of direct versus indirect drive continues on. 


\subsection{Ion Beam Driven Inertial Fusion}

Ion beam driven inertial fusion offers significant economic advantages to other confinement processes. Additionally, it offers the attractiveness of higher efficiencies, high repetition rates, and higher resistance to radiation damage than other inertial confinement methods. Ion beams of numerous species can be used to drive fusion targets, but the components of light ion drivers would significantly differ from those of heavy ions. Lighter ion drivers require energies $\geq 10 \mathrm{MeV}$ and a total current $\leq 10$ MA while heavy ion drivers require energies $\leq 10 \mathrm{GeV}$ and a total current $\geq 10 \mathrm{kA}$. The light ion drivers require more magnetic focusing and less acceleration schemes than the heavy ion drivers.

The basic components of an ion beam driver for inertial fusion are multifaceted (Fig. 1.6). The accelerator would consist of many beams. Each beam would require a low-emittance ion source capable of achieving normalized emittances below $1 \pi \mathrm{mm}$ mrad. This low emittance beam would be accelerated and injected into a matching section with a current and voltage of $\geq 100 \mathrm{~mA}$ and $\geq 1 \mathrm{MeV}$ depending on the mass of the ion species. After matching the space charge of the beam, the beam would be focused and accelerated in parallel. While the beam is being accelerated from $\geq$ $1 \mathrm{MeV}$ to $10 \mathrm{MeV}<\mathrm{E}<10 \mathrm{GeV}$ (depending on the ion species) it would also be axially compressed from $\mathrm{a} \sim 10-\mu \mathrm{s}, \geq 100-\mathrm{mA}$ bunch to a $\sim 100-\mathrm{ns} \geq 10$-A bunch. Combining beams could further increase the current. Once the final energy of the beam is achieved the beam bunch undergoes one last axial compression and focusing stage. These beam bunches may require neutralization by a background plasma if the final currents are not high enough to provide self focusing. This closing stage of axial compression will increase the current of the combined bunch to $\geq 1 \mathrm{kA}$. 


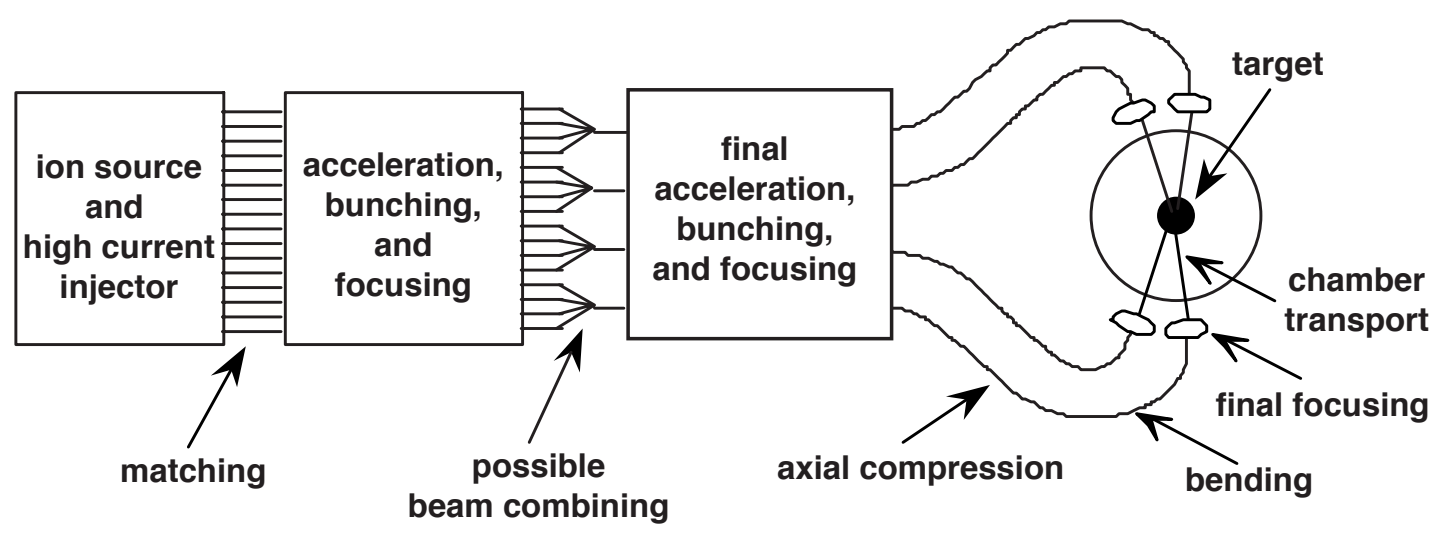

Figure 1.6. Ion accelerator components necessary for IFE.

\subsection{Warm Dense Matter (WDM) target heating experiments}

Warm dense matter (WDM) is the non-equilibrium state of matter between a solid and a plasma. It is also be referred to as the region in temperature $(\mathrm{T})$ - density $(\rho)$ space defined as $0.1 \mathrm{eV}<\mathrm{T}<10 \mathrm{eV}$ and $0.1 \rho_{\mathrm{o}}<\rho<10 \rho_{\mathrm{o}}$ where $\rho_{\mathrm{o}}$ is solid density (Fig. 1.7). WDM is a state of matter too dense to be described by weakly-coupled plasma physics and too energetic to be described by condensed matter physics.

In this state it is assumed that the ratio of the potential energy of the interaction between particles $\left(V_{i i}\right)$ and the kinetic energy of these particles $(T)$, or the strong coupling parameter $(\Gamma)$ varies significantly. The strong coupling parameter can be defined in terms of the ionization state $(Z)$, electron charge $(e)$, and radius or thickness of the selected material $(r)$ below:

$$
\Gamma=\frac{V_{i i}}{T}=\frac{Z^{2} e^{2}}{r T}
$$

The purpose of studying materials in the WDM Regime is to better explain the Equation of State (EOS). WDM is expected in the cores of some large planets and 


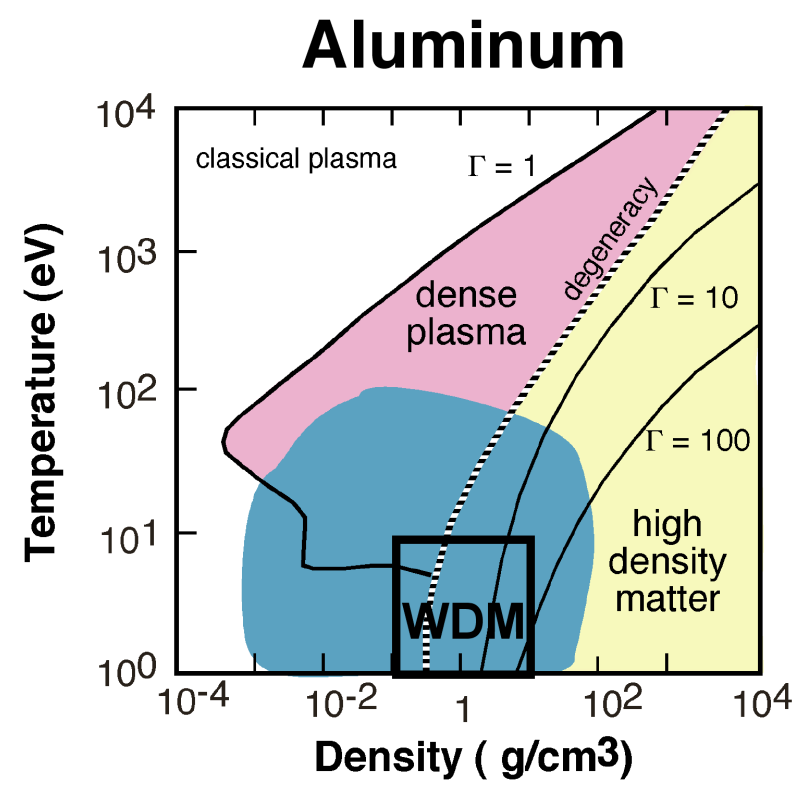

Figure 1.7. Equation of State diagram for Aluminum displaying different curves for the strong coupling parameter. The region of WDM is marked by a black border.

stars, inertial fusion energy implosions, and other systems that start as solids and are heated to become plasmas [5]. Therefore, it is an area of interest to many scientific disciplines.

WDM on earth is recreated in the laboratory through intense laser-target interactions [6] and particle beam-target interactions [7]. One of the basic requirements of the interactions is that the length of the pulse, which is heating the target, must be much longer than the local thermodynamic equilibrium time $\left(t_{\text {LTE }}\right)$ and much less than the hydrodynamic disassembly time $\left(t_{\text {hydro }}\right)$.

One of the advantages of using ions to heat these targets is uniform heating and energy deposition over the area which the ions are incident upon. The strategy is to deposit the ion energy in a target at or near the Bragg peak (peak $\mathrm{dE} / \mathrm{dx}$ ). Operating at the Bragg peak for the ion driver and target species precisely places the ion driver's energy at the desired location and heats the target to WDM by electronic stopping. 


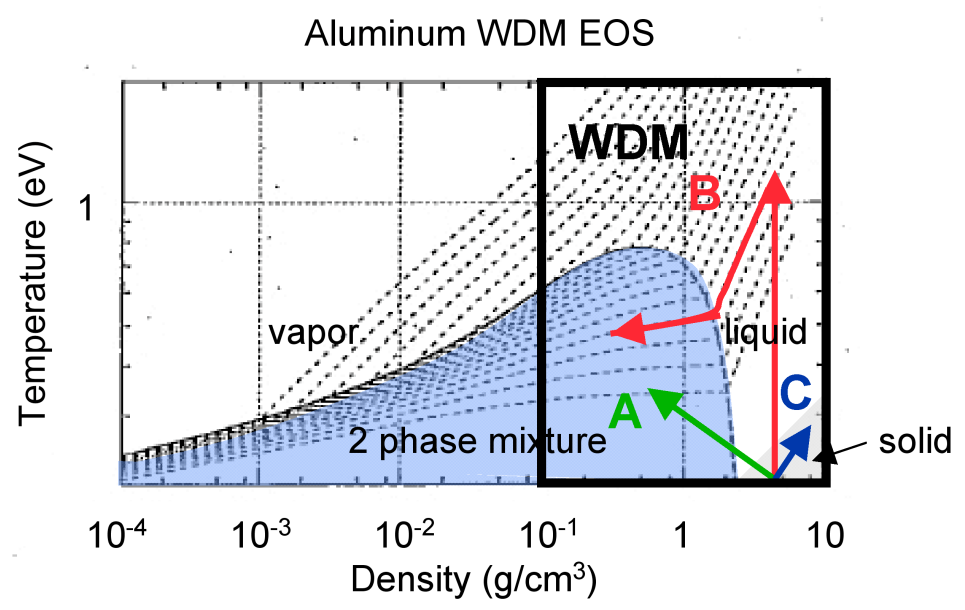

Figure 1.8. Equation of State diagram for Aluminum displaying different methods of changing solid Al to WDM. The region of WDM is marked by a black border.

Fig. 1.8 shows three different ways of heating an Aluminum target to WDM [8]. In method A, labeled in green, the target is heated slowly from solid Aluminum to a two phase mixture. In method B, labeled in red, the target is heated quickly from solid Aluminum to a liquid and then cools adiabatically to a two phase mixture. Method $\mathrm{B}$ is the proposed process of heating targets to WDM using ion drivers. In method C, labeled in blue, the target is shock heated and compressed from solid Aluminum to high density Aluminum. Method $\mathrm{C}$ is a typical process by which lasers heat targets to WDM.

\subsection{The Neutralized Drift Compression Experi- ment (NDCX)}

The Neutralized Drift Compression Experiment (NDCX) at Lawrence Berkeley National Laboratory is exploring the physical limits of compression and focusing of ion beams for heating material to Warm Dense Matter (WDM) and fusion ignition 
Table 1.1. Comparison of beam parameters for the front end of an Inertial Fusion Energy driver and the NDCX.

\begin{tabular}{|l|c|c|}
\hline & Ion Driver & NDCX \\
\hline & & \\
\hline Number of beams & 100 & 1 \\
\hline Ion mass (amu) & $>7$ & 39 \\
\hline Beam energy (MeV) & $>1$ & 0.4 \\
\hline Beam energy spread $(\mathrm{kV})$ & $<2$ & $<1$ \\
\hline$\Delta$ V/ V during pulse $(\%)$ & \pm 0.1 & \pm 1 \\
\hline Beam current per beam $(\mathrm{mA})$ & $>100$ & 70 \\
\hline$\Delta \mathrm{l} /$ I during pulse $(\%)$ & \pm 0.2 & \pm 1.0 \\
\hline Charge state purity & $>90 \%$ & $>99 \%$ \\
\hline Normalized Emittance $(4 \mathrm{rms} \pi \mathrm{mm}$ mrad) & $<1$ & 0.09 \\
\hline Beam pulse length $(\mu \mathrm{s})$ & 10 & 10 \\
\hline Beam rise time $(\mu \mathrm{s})$ & $<1$ & 0.5 \\
\hline Line charge density per beam $(\mathrm{nC} / \mathrm{m})$ & $>100$ & 50 \\
\hline Perveance & $10^{-3}$ & $10^{-3}$ \\
\hline
\end{tabular}

conditions [9-13]. The NDCX is a $\mathrm{K}^{+}$ion beam transport experiment with many of the same components as an Inertial Fusion Energy driver. The NDCX is an accelerator which consists of a low-emittance ion source, high-current injector, solenoid matching section, induction bunching module, beam neutralization section, and a final focusing system. The NDCX also has several parameters at a scale comparable to the front end of an Inertial Fusion Energy driver (Table 1.1).

The NDCX also has several parameters at a scale comparable to a future ion accelerator capable of heating targets to $\mathrm{T}_{\mathrm{e}}>1 \mathrm{eV}$ (Table 1.2). The NDCX is a lower energy accelerator, so it cannot heat targets at the Bragg peak, yet is still capable of depositing enough energy $\left(\sim 0.1 \mathrm{~J} / \mathrm{cm}^{2}\right)$ to produce temperatures in the WDM regime $(\sim 0.1 \mathrm{eV})$ 
Table 1.2. Comparison of beam parameters for NDCX and a future WDM accelerator.

\begin{tabular}{|l|c|c|}
\hline & WDM Driver & NDCX \\
\hline & & \\
\hline Number of beams & 1 & 1 \\
\hline lon mass (amu) & 7 & 39 \\
\hline Beam energy (MeV) & 2.9 & 0.3 \\
\hline Initial Current $(\mathrm{mA})$ & 100 & 30 \\
\hline Final Current $(\mathrm{A})$ & 30 & 3 \\
\hline Initial Pulse Duration $(\mathrm{ns})$ & 300 & 200 \\
\hline Final Pulse Width $(\mathrm{ns})$ & $<1$ & 2 \\
\hline Beam density at focus $\left(\mathrm{cm}^{-3}\right)$ & $>10^{13}$ & $>10^{13}$ \\
\hline Line charge density at focus $(\mu \mathrm{C} / \mathrm{m})$ & 3.6 & 2.5 \\
\hline Target thickness $(\mu \mathrm{m})$ & 3 & $<1$ \\
\hline Percent solid density & $100 \%$ & $10 \%$ \\
\hline Target temperature $(\mathrm{eV})$ & 2 & 0.1 \\
\hline Energy deposition $\left(\mathrm{J} / \mathrm{cm}^{2}\right)$ & 20 & 0.1 \\
\hline
\end{tabular}

\subsubsection{NDCX objectives}

The principal objectives of the experiment are to control the beam envelope, demonstrate effective neutralization of the beam space-charge, control the velocity tilt on beam, and understand defocusing effects, field imperfections, and limitations on peak intensity such as emittance and aberrations.

\subsubsection{Experimental configurations}

There were several different experimental configurations used on the NDCX to study the different physics questions. The $\mathrm{K}^{+}$ion beam used for all of the experiments on the NDCX was accelerated through a 12-cm long diode and extracted through a 4-cm diameter aperture (Fig. 1.9). Two cylindrical electron suppression electrodes, with a removable current reducing aperture, followed directly downstream. Transverse beam dynamics measurements were made to characterize the injected beam between 15 and $31 \mathrm{~cm}$ downstream of the exit of the diode before installing the solenoids. The 


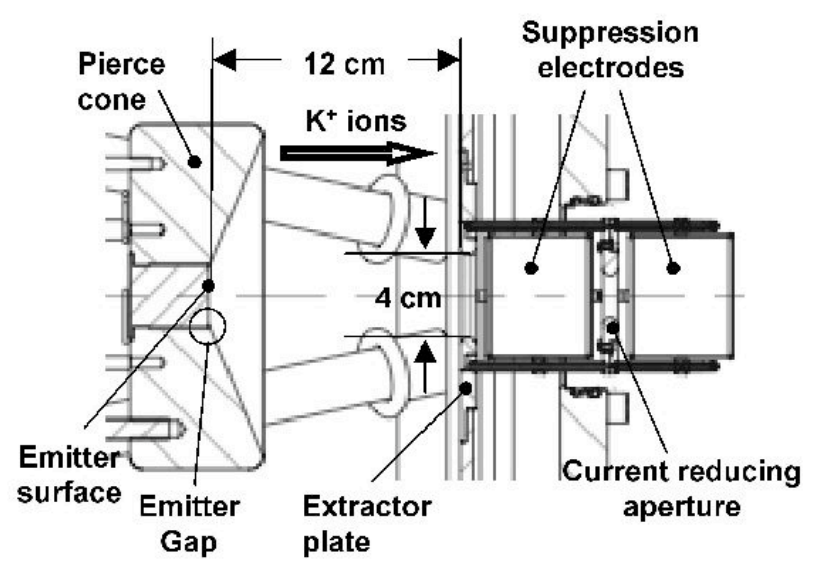

Figure 1.9. Diagram of the Pierce diode geometry and suppression electrodes with removable aperture downstream.

fully extracted beam current at $300 \mathrm{kV}$ was $45 \mathrm{~mA}$ and installing a 2-cm aperture reduced the current to $26 \mathrm{~mA}[12]$.

After characterizing the beam extracted from the diode, two solenoids were placed on the NDCX beamline immediately downstream of the diode (Fig. 1.10). The focusing lattice consisted of two $50-\mathrm{cm}$ solenoids spaced about $9 \mathrm{~cm}$ apart with a diagnostic box at the exit of the second solenoid [14]. An additional cylindrical electrode was added at the exit of the last solenoid, upstream of the intercepting diagnostics, to suppress any electrons from backstreaming into the solenoid lattice.

After characterizing the matching and transport of the beam through two solenoids, two more solenoids were added to the NDCX beamline (Fig. 1.11). The two additional solenoids were also 50-cm long, had an identical construction to those used in the two-solenoid experiments, and the spacing in between all of the magnets was about $9 \mathrm{~cm}[13]$.

Extensive studies of electron cloud and gas effects in the four-solenoid lattice were done with the apertured 26-mA beam using new cylindrical electrodes inside the beam pipe (electron cloud diagnostics; Fig. 1.11). These electron cloud diagnostics 


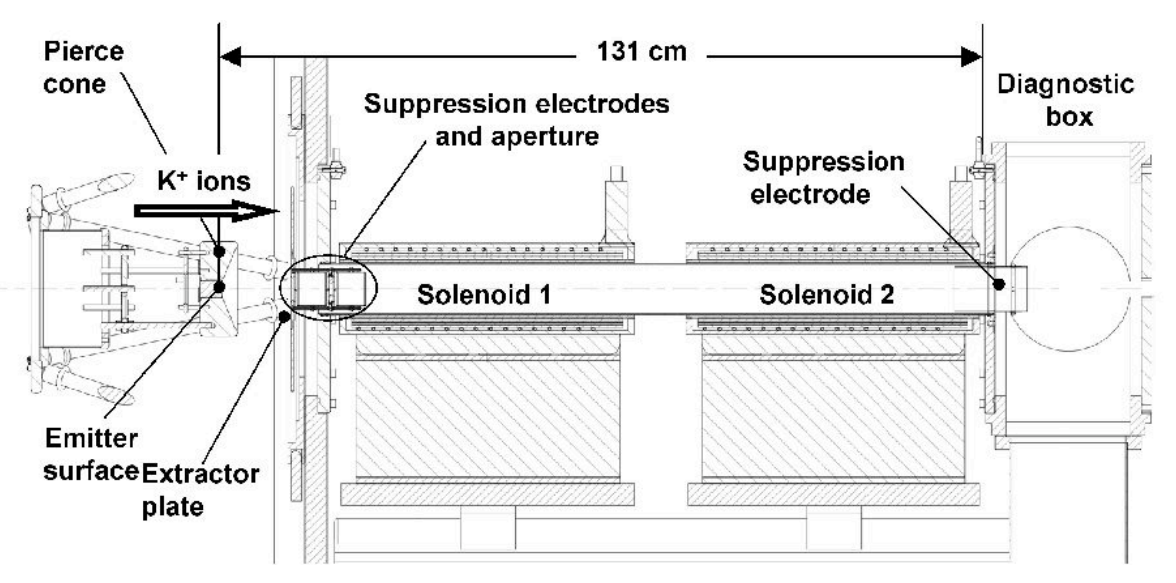

Figure 1.10. Elevation view of the Two-Solenoid Experiment.

consisted of four short $(8.45-\mathrm{cm}$ long) cylindrical electrodes in the center of each solenoid magnet (solenoid electrodes 1, 3, 5, and 7) and the three longer (25.4-cm long) cylindrical electrodes in the gaps between magnets (gap electrodes 2, 4, and 6). These electrodes have a radius $(3.6 \mathrm{~cm})$ slightly smaller than the beam pipe radius of $4.3 \mathrm{~cm}$. The gap electrodes were strategically placed to intercept the maximum amount of expanding magnetic flux between magnets (Fig. 1.11). The 13-cm long cylindrical electrode that was just upstream of the intercepting diagnostics in Fig. 10 was moved downstream into the exit of solenoid 4 (electrode 8; Fig. 1.11) to intercept the expanding magnetic flux at the exit of solenoid 4, making electrode 8 have a similar function to a gap electrode. A pair of parallel plates was used in place of this cylindrical electrode just upstream of the intercepting diagnostics to suppress electrons and measure the dynamics of beam-induced gas desorption, ionization, and electron emission. The measurements were also used to benchmark electron cloud models and codes.

Once electron cloud and gas measurements were completed, studies of combined transverse and longitudinal focusing of a $0.3-\mathrm{MeV}, 26-\mathrm{mA}$ singly charged $\mathrm{K}^{+}$ion beam were conducted on the NDCX as shown in Fig. 1.12 [11]. The induction bunching 


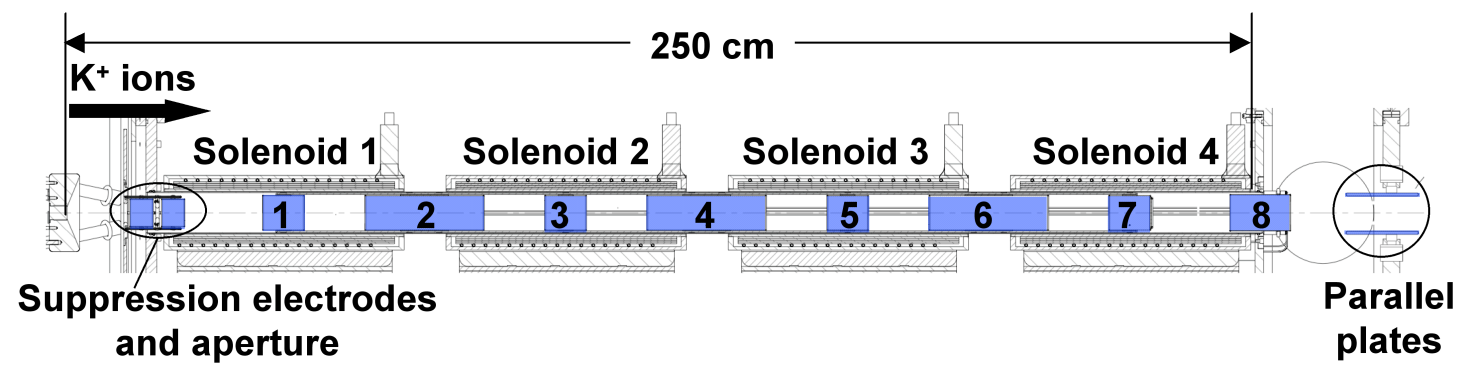

Figure 1.11. Layout of the aperture and suppression electrodes, electron cloud diagnostics: solenoid electrodes (1, 3, 5, and 7), gap electrodes (2, 4, 6, and 8); and parallel plate diagnostic relative to the four-solenoid lattice. All the diagnostics have cylindrical symmetry except for the parallel plate diagnostic.

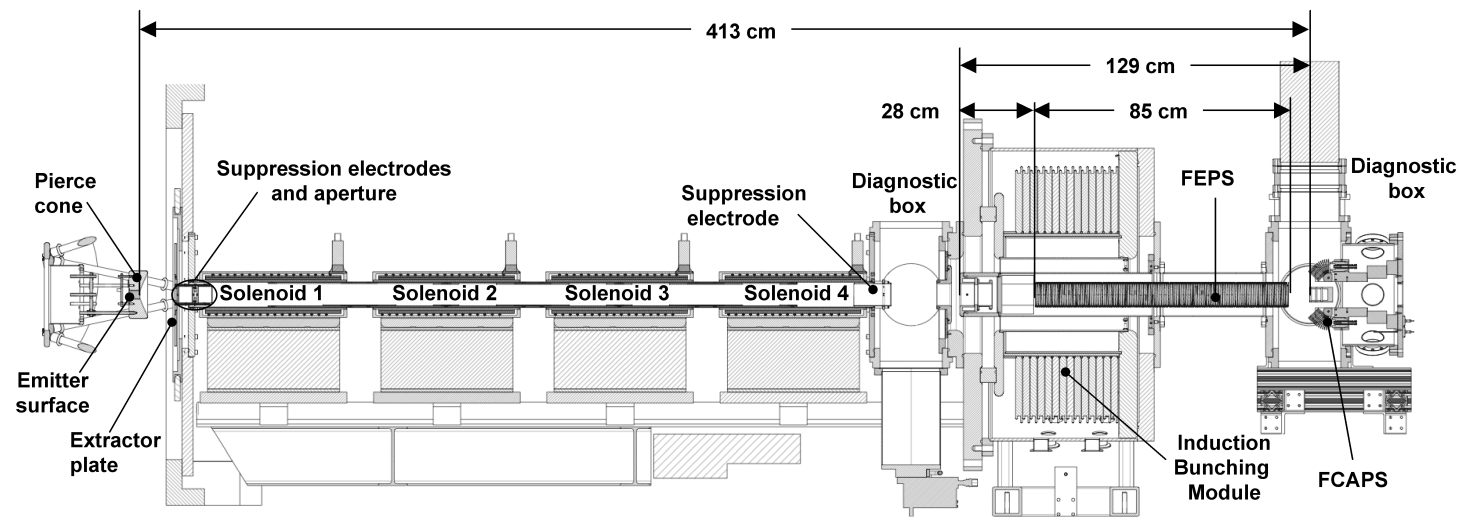

Figure 1.12. Elevation view of the Simultaneous Focusing Experiment on NDCX.

module (IBM) was located downstream of a beam diagnostic box located at the exit of the 4-solenoid lattice. The 4-solenoid transport lattice was used to match the beam to the desired envelope parameters $\left(\mathrm{a}=15 \mathrm{~mm}, \mathrm{a}^{\prime}=-30 \mathrm{mrad}\right)$ at the entrance to the IBM. The IBM provided a linear velocity ramp $(\Delta \mathrm{v} / \mathrm{v} \sim \pm 15 \%)$ on a $200 \mathrm{~ns}$ portion of the injected beam and was tuned specifically for the beam energy and a drift distance of $1.29 \mathrm{~m}$. Plasma neutralization began $28 \mathrm{~cm}$ downstream with an 85-cm long ferro-electric plasma column (FEPS) [15]. The fully neutralized beam then drifted $16 \mathrm{~cm}$ to the focal plane. A filtered cathodic arc plasma source (FCAPS) was also used for neutralizing the beam at the diagnostic plane [16]. 


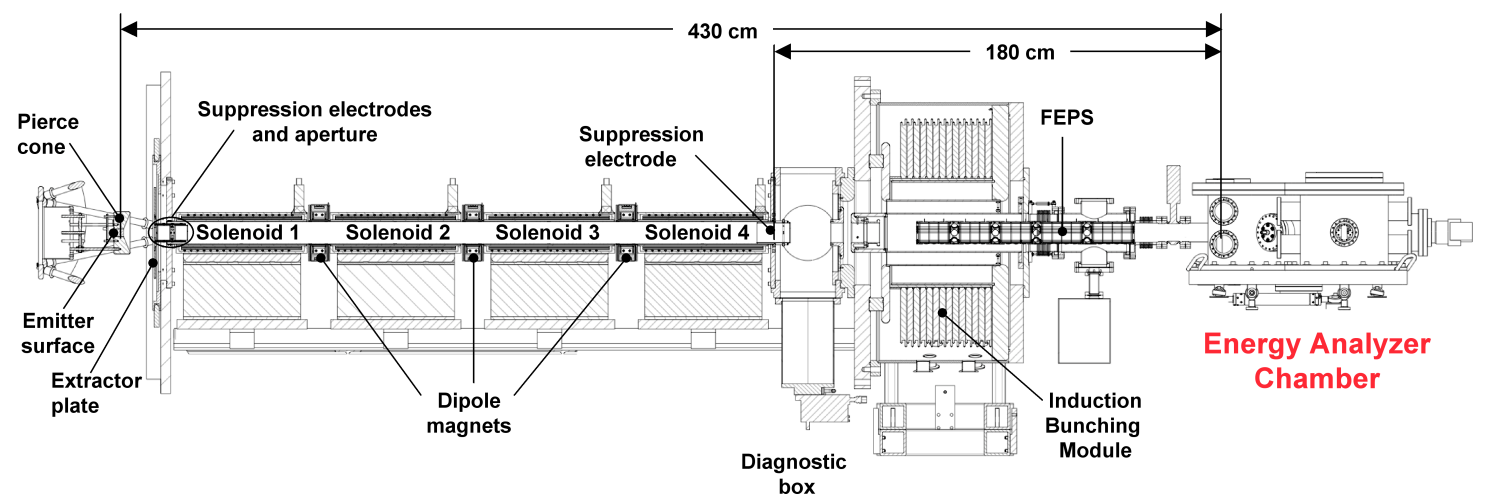

Figure 1.13. Elevation view of the NDCX with the EEA added on at the exit of the FEPS.

An additional configuration was used to study the limits of axial compression. An electrostatic energy analyzer (EEA) was added at the exit of the FEPS (Fig. 1.13) to measure the longitudinal phase space and temperature of the beam with and without plasma. The beam passes through a $0.1 \mathrm{~mm} \times 10 \mathrm{~mm}$ slit plate at the entrance to the spectrometer. This slit reduces the transmitted beam current from $26 \mathrm{~mA}$, which is incident on the slit plate, to $\sim 1 \mu \mathrm{A}$. The beam ribbon traversed the $90^{\circ}$ bend of the electrostatic dipole and was detected at the focal plane.

\subsubsection{Results reported in this thesis}

The remainder of this thesis is organized as follows: In Chapter 2, we present the relevant charged particle physics for the data presented in later chapters. In Chapter 3, all the diagnostics used to acquire the data are described, in addition to how data is presented in later chapters. Chapter 4 describes different numerical simulation tools used for comparison to the results of the experiment. Then, we present results of beam characterization after injection, matching, and transport in Chapter 5. The impact of electron cloud effects and beam centroid motion on the beam quality and dynamics is also addressed. In Chapter 6 we present achievements of simultaneous 
beam focusing and bunching. The physics that limit the intensity are also discussed. In Chapter 7 we discuss longitudinal temperature and phase space measurements with a high-resolution electrostatic energy analyzer.

Conclusions are drawn based on the results of this thesis in Chapter 8. The physical limitations of matching and transport are described which include the impact of electron cloud effects and beam centroid motion on the beam quality and dynamics. The achievements of simultaneous bunching and focusing are also mentioned in addition to possible improvements. The improvements are folded into future recommendations and include diagnostic improvement in addition to the use and development of additional hardware. 


\section{Chapter 2}

\section{Relevant charged particle physics}

\section{$2.1 \quad$ Introduction}

This chapter outlines the relevant physics necessary for understanding the research described in this thesis. More detailed explanations of these topics are found in Refs. [17-22]. Section 2.1 introduces the coordinate system, definition of a plasma, charged particle beams, and single particle motion.

\subsubsection{Coordinate system and units}

We use the right handed Cartesian coordinate system to describe the configuration space of the charged particles and beam in this thesis. The axis of propagation of the particles is labeled as the z-axis [Fig. 2.1(a)], vertically up is the y-axis, horizontally to the right with the axis of propagation coming out of the page is the x-axis [Fig. 2.1(b)], and $\phi$ is the azimuthal coordinate. The units used in this thesis are the International System of Units (SI) or the MKS system. 


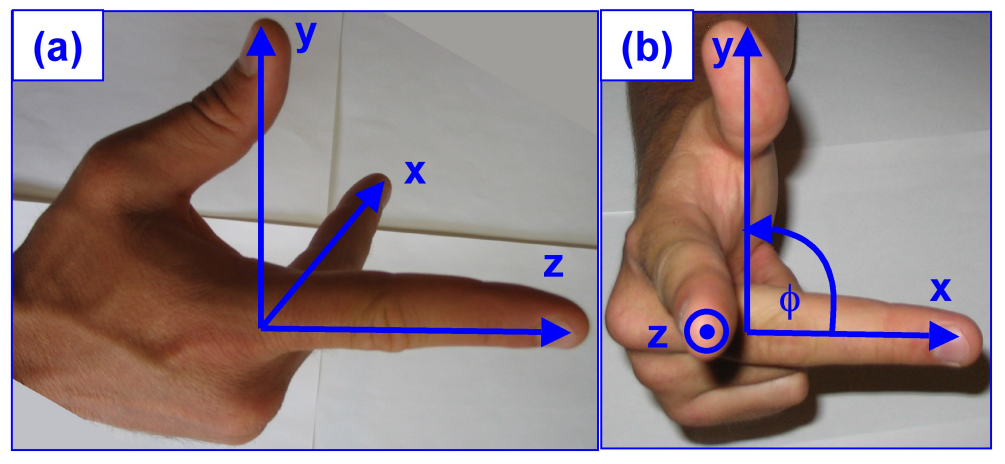

Figure 2.1. Pictures of the right hand coordinate system with axes labeled. (a) A view from the left side with the z-axis propagating from left to right and; (b) a view head on with the $\mathrm{z}$-axis coming out of the page.

\subsubsection{Plasmas}

A plasma is considered a distinct state of matter, the fourth state of matter, because of its unique properties. It is a superheated gas where one or more electrons become separated from the atom (or gas atoms become ionized). A plasma is not simply any ionized gas, but must display some collective behavior or be quasineutral. A plasma is a sea of charged particles, in which the kinetic energy of a given particle is generally greater than its potential energy with respect to its nearest neighbor (weakly coupled). The free electric charges make the plasma electrically conductive so that it responds strongly to electromagnetic fields.

\subsubsection{Charged particle beams}

A charged particle beam is a spatially localized group of electrically charged particles that have approximately the same velocity and direction. The kinetic energies of the particles in a given beam are dependent upon the energy distribution of the beam and whether the particles have been accelerated or decelerated. Typical particle beam energies are well above ambient temperatures in the range of a few eV to $\mathrm{TeV}$. 


\subsubsection{Single particle motion}

Electric and magnetic fields affect the orbit or direction of motion of a single particle. The general solution to a single particle's orbit is calculated by the equations laid out in this section [23, 24].

We begin with the force on a point charge $q$ in an electromagnetic field known as the Lorentz force

$$
\vec{F}=q(\vec{E}+\vec{v} \times \vec{B}),
$$

where $\vec{E}, \vec{v}$, and $\vec{B}$ are the electric field, velocity, and magnetic flux vectors. This equation is valid for static as well as time-varying fields and the fields obey Maxwell's equations (Eqs. 2.2). For our case the charged particles are in vacuum and $c^{2}=$ $1 / \epsilon_{o} \mu_{o}$, where $c$ is the speed of light, $\epsilon_{o}$ is the permittivity, and $\mu_{o}$ is the permeability of free space. Maxwell's equations are:

$$
\begin{gathered}
\vec{\nabla} \times \vec{E}=-\frac{\partial \vec{B}}{\partial t}, \\
\vec{\nabla} \cdot \vec{E}=\frac{\rho}{\epsilon_{o}}, \\
\vec{\nabla} \times \vec{H}=\vec{J}+\frac{\partial \vec{D}}{\partial t}, \\
\vec{\nabla} \cdot \vec{B}=0,
\end{gathered}
$$

where $\vec{D}=\epsilon_{o} \vec{E}$ and $\vec{B}=\mu_{o} \vec{H}$, and $\vec{D}$ and $\vec{H}$ are the electric flux and magnetic field vectors.

Assuming a particle in a uniform axial magnetic field, $B_{z}$, with a constant axial velocity, $v_{z}$, in the absence electric field it will see a force:

$$
\vec{F}=m \dot{v}=q \vec{v} \times \vec{B},
$$




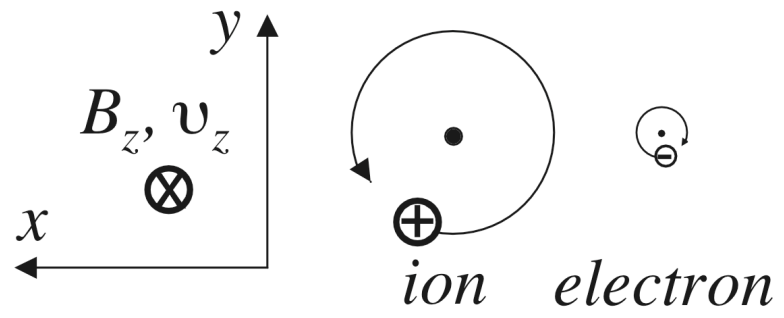

Figure 2.2. Sketch of the cyclotron motion of an ion and electron in a uniform axial magnetic field, $B_{z}$, with a constant axial velocity, $v_{z}$.

where $m \dot{v}_{x}=q v_{y} B, m \dot{v}_{y}=-q v_{x} B$, and $m \dot{v}_{z}=0$. This gives two equations of simple harmonic motion:

$$
\begin{aligned}
& \ddot{v}_{x}=-\left(\frac{q B}{m}\right)^{2} v_{y}, \\
& \ddot{v}_{y}=-\left(\frac{q B}{m}\right)^{2} v_{x},
\end{aligned}
$$

which have a solution $v_{x}=v_{y}=v_{\perp} \exp \left( \pm i \omega_{c} t+\phi\right)$ where the particle rotates at a frequency known as the cyclotron frequency or gyrofrequency.

$$
\omega_{c}=\frac{q B}{m}
$$

The radius at which the particle rotates about the magnetic flux lines is defined as the gyroradius:

$$
r_{c}=\frac{v_{\perp}}{\omega_{c}}
$$

Assuming positive ions and electrons have the same perpendicular velocity in the same magnetic field the electrons will rotate at a higher frequency and smaller radius proportional to the ion-electron mass ratio. For a magnetic field going into the page the ions will rotate counterclockwise and the electrons will rotate clockwise (Fig. 2.2). 


\subsection{Plasma sheaths and parameters}

In this section we present cases relevant to situations encountered by plasma based diagnostics used on NDCX. We define plasma parameters such as the effective length in a plasma sheath, its oscillation frequency, and other sheath characteristics.

\subsubsection{Debye length}

We start with Poisson's equation relating the electric potential $V$ to the volume charge density $\rho$ due to electrons and ions [25]

$$
\nabla^{2} V=-\frac{\rho}{\epsilon_{o}}=-\frac{q}{\epsilon_{o}}\left(n_{i}-n_{e}\right)
$$

where $q$ is the electric charge and $n_{i}$ and $n_{e}$ are the ion and electron particle densities. We can use Boltzmann's relation for electrons and ions, the variation of a particle's density $n(x)$ across a medium with a varying potential $V(x)$ in one dimension

$$
n(x)=n_{o} e^{\frac{V(x)}{T}},
$$

where $T$ is the particle temperature in volts. Assuming a negative plate is immersed into an infinite, one-dimensional plasma medium we substitute Boltzmann's relation for electrons $n_{e}(x)=n_{o} \exp \left(V(x) / T_{e}\right)$, assume the ions are fixed at a density of $n_{o}$, and $V \ll T_{e}$, Poisson's equation simplifies to

$$
\frac{d^{2} V(x)}{d x^{2}}=\frac{q n_{o} V(x)}{\epsilon_{o} T_{e}} .
$$

The potential variation across the medium is

$$
V(x)=V_{o} e^{-x \sqrt{\frac{n_{o} q}{\epsilon_{o} T_{e}}}} .
$$

This negative plate repels electrons and a positive sheath of ions forms to shield the rest of the plasma from the negative potential applied to the plate. The characteristic 
length scale of the sheath formed in the plasma, the electron Debye length $\lambda_{D e}$, is defined by extracting the constants from the equation above

$$
\lambda_{D e}=\sqrt{\frac{\epsilon_{o} T_{e}}{n_{o} e}} .
$$

\subsubsection{Plasma frequency}

A similar sheath is formed with electrons using a positively biased plate. If one has both a positive and negative plate, an ion and electron sheath will form and these sheaths or clouds will have a sinusoidal oscillation with respect to one another [26]. By solving Lorentz force Eq. (2.1) or the equation of motion for stationary ions and electrons without an induced magnetic field, the frequency of oscillation, or the plasma frequency $\omega_{p}$ can be defined as

$$
\omega_{p}=\sqrt{\frac{n q^{2}}{\epsilon_{o} m}} .
$$

The Debye length $\lambda_{D}$ and plasma frequency $\omega_{p}$ of a particle species are related to one another by the particles thermal velocity $v_{t h}$.

$$
\lambda_{D}=\frac{v_{t h}}{\omega_{p}} .
$$

\subsubsection{The high-voltage sheath}

In a simple high-voltage sheath, where the potential $V$ is highly negative, there are only ions in the sheath [27]. We assume the ion density is constant $\mathrm{n}_{i}(\mathrm{x})=\mathrm{n}_{o}$ and Poisson's Eq. (2.7) simplifies to

$$
\nabla^{2} V=-\frac{\rho}{\epsilon_{o}}=-\frac{q n_{o}}{\epsilon_{o}}
$$

The electric field $\vec{E}$ can be solved for

$$
\vec{E}(x)=\frac{q n}{\epsilon_{o}} x,
$$


and the voltage profile of the sheath can be found by integration

$$
V(x)=-\frac{q n x^{2}}{2 \epsilon_{o}} .
$$

Setting the peak voltage at the edge of the sheath to - $V_{o}$ (plate voltage) we can solve for the sheath thickness $\delta \mathrm{x}$

$$
\delta x=\sqrt{\frac{2 \epsilon_{o} V_{o}}{q n}} .
$$

Remembering the electron Debye length, $\lambda_{D e}$, from Eq. (2.11) we obtain

$$
\delta x=\lambda_{D e} \sqrt{\frac{2 V_{o}}{T_{e}}} .
$$

We see the high voltage sheath thickness $\delta \mathrm{x}>\lambda_{D e}$ if the plate voltage $V_{o} \gg$ the electron temperature $T_{e}$. This relation is useful for high voltage probes used to measure plasma density and beam current in a plasma environment as will be explained in Chapter 3.

\subsubsection{The 1-D Child Law}

On the NDCX we operate the emitter at high temperatures $\left(\mathrm{T} \sim 1000^{\circ} \mathrm{C}\right)$ to ensure we are extracting the beam at the space charge limit. This current extraction through the diode is similar to a non-relativistic, one dimensional, high-voltage sheath and should obey the 1-D Child Law. The initial ion energy $E_{o} \ll$ the potential $V_{o}$; the ion energy $E(x)$ and current density $J$ are defined below:

$$
\begin{gathered}
E(x)=\frac{1}{2} m v^{2}(x)=-q V(x), \\
J=q n(x) v(x),
\end{gathered}
$$

where $v(x)$ is the ion velocity. Solving for the ion density $n(x)$ we obtain

$$
n(x)=\frac{J}{q}\left(\frac{-2 q V}{m}\right)^{-\frac{1}{2}} \text {. }
$$


Substituting this into Poisson's Eq. (2.14) we have

$$
\nabla^{2} V(x)=-\frac{J}{\epsilon_{o}}\left(\frac{-2 q V}{m}\right)^{-\frac{1}{2}} .
$$

If we multiply Eq. (2.22) by $d V(x) / d x$ and integrate, we obtain

$$
\frac{1}{2}\left(\frac{d V(x)}{d x}\right)^{2}=\frac{2 J}{\epsilon_{o}}\left(\frac{2 q}{m}\right)^{-\frac{1}{2}} \sqrt{-V},
$$

by setting $d V(x) / d x=-\mathrm{E}(0)=0$ and $\mathrm{V}(0)=0$. Then taking the negative square root, integrating Eq. (2.23), setting $V(x)=V_{o}$, and solving for $J$ we get the 1-D Child Law

$$
J=\frac{4 \epsilon_{o}}{9} \sqrt{\frac{2 q}{m}} \frac{V^{3 / 2}}{d^{2}},
$$

where $J$ is the current density of the beam, $\epsilon_{o}$ is the permittivity of free space, $q$ is the charge of the particle species, $m$ is the mass of the particle species, $V$ is the voltage applied across the diode, and $d$ is the diode gap. From Eq. (2.24) a relation is developed between the current extracted and the voltage used in the gun, or the perveance of the gun, $K_{\text {gun }}$

$$
K_{\text {gun }}=\frac{I}{V^{3 / 2}}
$$

where $K_{\text {gun }}$ is a constant that is a function of the gun geometry, and the beam charge and mass.

\subsection{Transverse charged particle beam dynamics}

The beams used on the NDCX and most particle accelerators are considered paraxial because the transverse motion is always much smaller than the axial motion. However, we study the motion in both the transverse and axial planes. In this section we will present the transverse beam dynamics relevant to the experiments conducted on the NDCX. 


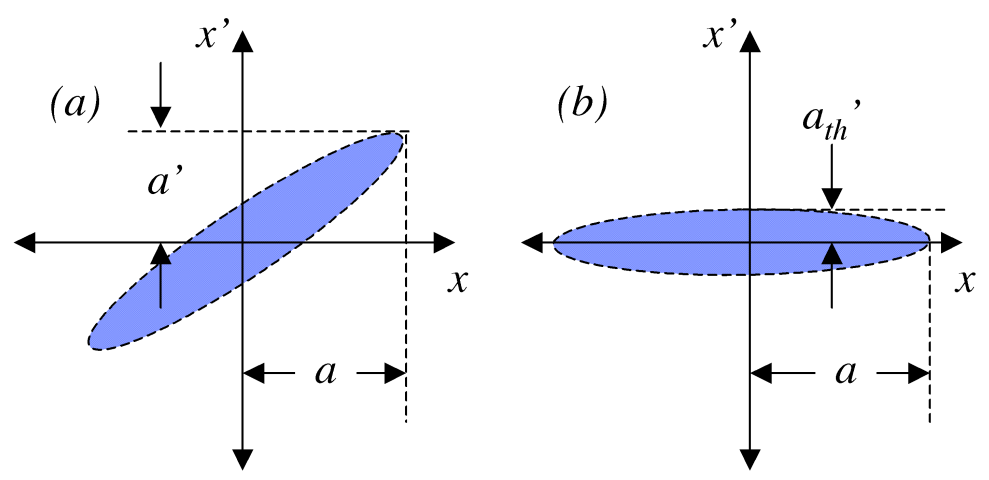

Figure 2.3. Sketch of the (a) transverse phase space; and (b) the sheared phase space distributions.

\subsubsection{Transverse phase space and emittance}

For non-relativistic particles, phase space is a six dimensional space with 3 position coordinates $(\mathrm{x}, \mathrm{y}$, and $\mathrm{z})$ and 3 velocity coordinates $\left(\mathrm{v}_{x}, \mathrm{v}_{y}\right.$, and $\left.\mathrm{v}_{z}\right)$ [28-31]. The number of particles $N$ in a volume element $d V$ of phase space or the density of particles $n$ is a constant of motion. This is known as Liouville's theorem. This conservation of 6-D phase space can be broken down into individual 2-D phase space elements, the $x$ and $y$ transverse components and the $z$ or longitudinal component. There are linear couplings between each of these phase space elements however the number of particles in $\mathrm{x}-\mathrm{v}_{x}, \mathrm{y}-\mathrm{v}_{y}$, and $\mathrm{z}-\mathrm{v}_{z}$ phase space are all usually measured individually.

An example of a uniform distribution in 2-D $\mathrm{x}-\mathrm{x}^{\prime}$ transverse phase space is shown in blue in Fig. 2.3. Instead of $v_{x}$ we use $x^{\prime}$, the transverse angle coordinate, which is normalized to $v_{z}$ to show small changes in the transverse envelope for non-accelerating paraxial beams.

$$
x^{\prime}=\frac{v_{x}}{v_{z}}
$$

An effective area or ellipse is drawn around the distribution in Fig. 2.3 to demon- 
strate the calculated emittance. Emittance is a conserved quantity for ideal (well aligned, linear) focusing systems and is considered a measure of the effective volume occupied by a distribution or the quality of a beam. For a beam with little current or where the space charge has been neutralized the transverse envelope and emittance of the beam are what physically limit the beam from approaching an infinitesimally small spot. Mathematically emittance is defined as:

$$
\epsilon_{4 r m s}=4 \sqrt{\left\langle x^{2}\right\rangle\left\langle x^{\prime 2}\right\rangle-\left\langle x x^{\prime}\right\rangle^{2}}
$$

where $\left\langle\mathrm{x}^{2}\right\rangle$ and $\left\langle\mathrm{x}^{\prime 2}\right\rangle$ are the second moments of the distribution and the third term underneath the square root is the correlation term which accounts for the expansion and contraction of the phase space ellipse. If $\left\langle\mathrm{xx}^{\prime}\right\rangle=0$ then the $4 \mathrm{rms}$ emittance is defined as:

$$
\epsilon_{4 r m s}=a a_{t h}^{\prime}
$$

which is the initial condition at the emitter. The units for each of the above definitions are $\pi \cdot \mathrm{mm} \cdot \mathrm{mrad}$. $a$ and $a^{\prime}$ are the $2 \mathrm{rms}$ values or statistical averages of the beam radius and angle, and $a_{t h}^{\prime}$ is the $2 \mathrm{rms}$ thermal spread of the beam.

$$
\begin{aligned}
& a=2 \sqrt{\left\langle x^{2}\right\rangle}, \\
& a^{\prime}=2 \sqrt{\left\langle x^{\prime 2}\right\rangle} .
\end{aligned}
$$

The normalized emittance, defined below, is ideally conserved for accelerating beams.

$$
\epsilon_{n 4 r m s}=\beta \gamma \epsilon_{4 r m s}
$$

where $\beta=v_{z} / c$ and $\gamma$ is defined as:

$$
\gamma=\frac{1}{\sqrt{1-\beta^{2}}}
$$


and can be approximated as

$$
\gamma=\frac{E}{E_{o}},
$$

where $E$ is the total energy of a particle and $E_{o}=m_{o} c^{2}$ is the rest energy of a particle.

The distribution in Fig. 2.3(b) is sheared, meaning the transverse envelope angle $a^{\prime}$ has been removed to show the thermal spread, $a_{t h}^{\prime}$, of the beam.

$$
\begin{gathered}
a_{t h}^{\prime}=\frac{\Delta v_{x}}{v_{z}}=\frac{v_{T x}}{v_{z}}, \\
\Delta v_{x}=v_{T x}=\sqrt{\frac{T_{x}}{m}},
\end{gathered}
$$

where $\Delta v_{x}$ is the rms spread in the transverse velocity, $v_{T x}$ is the transverse thermal velocity, $T_{x}$ is the transverse beam temperature in $\mathrm{eV}$, and $m$ is the mass in $\mathrm{eV} / \mathrm{c}^{2}$. The normalized thermal emittance for an ion source can be calculated as:

$$
\epsilon_{n}=2 \pi r v_{T x}
$$

where $r$ is the radius of the emitter.

When $a$ is very large and has a large converging or diverging angle $a^{\prime}$ its thermal width $a_{t h}^{\prime}$ or thickness in phase space will be very narrow. The opposite will be the case if $a$ is very small and the beam is at a waist. A laminar beam or very cold beam has nearly zero thickness, and the thermal component or emittance approaches zero $\left(\mathrm{a}^{\prime}{ }_{t h} \rightarrow 0\right)$.

\subsubsection{Transverse envelope equation}

The transverse envelope equation describes the evolution of the transverse beam size subject to externally applied and beam self fields. The transverse envelope equation below is derived from the equation of motion while assuming radially and axially 
symmetric fields [32, 33]. There are applied radial and axial electric and magnetic fields $E_{a}(r, z), B_{a}(r, z)$, radial electric fields due to the beam space charge $E_{s}(r)$, and azimuthal magnetic field induced by the beam current $B_{s}(\phi)$, where the subscripts $a$ and $s$ denote the applied and self fields.

$$
R^{\prime \prime}=-\frac{\gamma^{\prime} R^{\prime}}{\beta^{2} \gamma}-\frac{\gamma^{\prime \prime} R}{2 \beta^{2} \gamma}-\left(\frac{q B_{z}}{2 \beta \gamma m c}\right)^{2} R+\frac{\epsilon^{2}}{R^{3}}+\left(\frac{p_{\phi}}{\beta \gamma m c}\right)^{2} \frac{1}{R^{3}}+\frac{K}{R} .
$$

The first term $R^{\prime \prime}$ is the second derivative of the radius with respect to the axis of propagation. The second term is a focusing term due to acceleration from axial electric fields. The third term is a focusing term due to radial components of applied electric fields.

The fourth term is a focusing term due to an applied axial magnetic field $\left(\mathrm{B}_{a}(\mathrm{z})\right)$ provided by a focusing solenoid which will be derived later in Section 2.3.3. It can be written in a slightly different form for other magnetic focusing elements such as magnetic quadrupoles, sextupoles, but in the interest of this thesis we will only consider solenoids. The focusing strength $\kappa$ in Eq. (2.37) for solenoids can be defined as:

$$
\kappa=\left(\frac{q B_{z}}{2 \beta \gamma m c}\right)^{2}
$$

The fifth term is a defocusing term due to the transverse emittance or the thermal spread of the beam, where $\epsilon$ is the 4rms emittance defined in Eq. (2.27). For most of the beam transport on NDCX and ion beam drivers with high space charge this term is small unless the beam space charge is neutralized, then the emittance dominates.

The sixth term is a defocusing term due to canonical angular momentum $\mathrm{p}_{\phi}$. Canonical angular momentum is a conserved quantity and generally this term is negligible according to Busch's Theorem [34].

$$
p_{\phi}=\gamma m r^{2} \dot{\phi}+\frac{q}{2 \pi} \Phi=\text { constant },
$$


where $\dot{\phi}$ is the angular frequency of rotation, and $\Phi$ is magnetic flux. If the particle source is immersed in a magnetic field it will induce initial angular momentum. For the defocusing effect to be considered large enough $p_{\phi} / m c \geq \epsilon_{n}$, where $\epsilon_{n}$ is the normalized emittance from Eq. (2.31).

The seventh and final term is a defocusing term due to the electrostatic field of the beam. A beam has charge enclosed in a bunch and will therefore produce a radial beam potential, which can be found from Gauss's Law.

$$
\Psi=\int \vec{D} \cdot d \vec{a}=Q_{e n c}
$$

where $\Psi$ is the electric flux. Substituting $\vec{D}=\epsilon_{o} \vec{E}$ and the line charge density $\lambda$ the alternate form is

$$
\int \vec{E} \cdot d \vec{a}=\frac{1}{\epsilon_{o}} \int \lambda \cdot d \vec{z}
$$

The radial electric field can be solved for a long cylindrical beam (compared to its transverse size)

$$
E_{r}=\frac{\lambda}{2 \pi \epsilon_{o} r} \hat{r}
$$

and the radial beam potential $\mathrm{V}(\mathrm{r})$ can be found

$$
V(r)=-\int \vec{E} \cdot d \vec{r}=\frac{\lambda}{2 \pi \epsilon_{o}} \ln \left(\frac{a}{r}\right)
$$

where $a$ is the beam radius. The generalized perveance, $K$ in Eq. (2.37) is defined as the ratio of space charge forces to the inertial forces or the ratio of the potential energy of the beam to the kinetic energy of the beam.

$$
K=\frac{\int\left(E_{r}+v_{z} B_{\phi}\right) d r}{E}=\frac{q \lambda}{2 \pi \epsilon_{o} \gamma^{3} m v_{z}^{2}},
$$

where $E$ is the total kinetic energy and the line charge density $\lambda=I / v_{z}$. For nonrelativistic beams, which is the case on NDCX, the generalized perveance is

$$
K=\frac{q \lambda}{4 \pi \epsilon_{o} E}
$$




\subsubsection{Beam transport with solenoids}

Solenoids are used for beam transport on the NDCX. In this section the equations demonstrating matching and transport of charged particles with a solenoid are derived.

Ions emitted from a planar source with zero velocity and accelerated through a magnetic field free diode have a purely axial velocity, $v_{z}$, and no canonical momentum, $p_{\phi}$. Once these ions reach the fringe (radial) magnetic field, $B_{r}$ of solenoid $\left(B_{z} \gg B_{r}\right)$ they experience an azimuthal force $F_{\phi}$ from the Lorentz Force (Eq. 2.1).

$$
F_{\phi}=q v_{z} B_{r}
$$

which in turn gives the beam an azimuthal velocity component, $v_{\phi}$ in addition to $v_{z}$. This azimuthal velocity contributes to a radial focusing force, $F_{r}$, as the beam reaches the larger axial magnetic field $B_{z} \gg B_{r}$.

$$
F_{r}=q v_{\phi} B_{z}
$$

$F_{r}$ must balance or overcome the defocusing centrifugal, $F_{c e n t}$, and space charge forces $F_{S C}$ in order to match the beam.

$$
\begin{gathered}
F_{c e n t}=m \omega^{2} r \\
F_{S C}=\frac{K m v_{z}^{2} r}{a^{2}},
\end{gathered}
$$

where $\omega$ is the angular frequency of rotation of the beam. The force balance is written below after defining $v_{\phi}=\omega r$ in Eq. (2.47)

$$
q \omega r B_{z}=m \omega^{2} r+\frac{K m v_{z}^{2} r}{a^{2}}
$$


Canceling $r$, dividing both sides of the equation by the mass $m$, and remembering the gyrofrequency from Eq. (2.5), the force balance becomes

$$
\omega_{c} \omega=\omega^{2}+K\left(\frac{v_{z}}{a}\right)^{2} .
$$

The beam becomes matched or reaches the Brillouin flow condition when $\omega$ is equal to the Larmor frequency $\left(\omega=\omega_{L}=\omega_{c} / 2\right)$. Brillouin flow is defined as solenoid transport of a laminar beam (zero emittance and uniform axial velocity) rotating at the Larmor frequency $\left(\omega_{c} / 2\right)$. The beam will see an effective rotation throughout the focusing lattice and the number of revolutions can be calculated as

$$
N_{\text {rev }}=\frac{\omega_{c} \Delta z}{4 \pi v_{z}},
$$

where $\Delta z$ is the distance traveled along the axis of propagation and $v_{z}$ is the axial velocity.

Assuming Brillouin flow, the maximum perveance $K_{\max }$ transportable through a solenoid channel is defined

$$
K_{\max }=\left(\frac{\omega_{c} a}{2 v_{z}}\right)^{2} .
$$

This is also found by simplifying the transverse envelope equation (Eq. 2.37) for a non-relativistic $(\gamma \sim 1, \beta \ll 1)$, continuously focused $\left(R^{\prime}=R^{\prime \prime}=0\right)$, space-charge dominated, laminar, coasting beam.

$$
\frac{K}{R}=\kappa R .
$$

The maximum line charge density $\lambda_{\max }$ of a particular ion species transportable through a solenoid channel is derived from Eqs. $(2.38,2.45,2.53,2.54)$ :

$$
\lambda_{\max }=\frac{\pi \epsilon_{o} q}{2 m}\left(B_{z} a\right)^{2} .
$$


The minimum field necessary to match a particular ion species can be derived from the above equation in terms of the density $n$ and mass $m$ of that species.

$$
B_{z}=\sqrt{\frac{2 n m}{\epsilon_{o}}} .
$$

The focal length $f$ of a focusing element like a solenoid is a function of the focusing strength $\kappa$ (Eq. 2.38) and the effective length of the focusing element $l$.

$$
f=(\kappa l)^{-1} .
$$

\subsubsection{Adverse effects on the transverse beam dynamics}

Up to this point all of the fundamental descriptions of beam behavior and limiting effects were assuming a well aligned, linear transport system. We will present results that are far from this ideal case in later chapters. Some of the effects seen in the results are due to causes which are not easily controlled in the laboratory.

One of the first adverse effects and possibly the most detrimental is the effect of misalignments. On the NDCX there are many components which must be aligned with precision ( $\ll 1 \mathrm{~mm}$ and $\ll 1 \mathrm{mrad}$ ) in order to yield ideal or acceptable conditions. If those components are not properly aligned the beam becomes distorted. Distortions in the beam distribution lead to emittance growth, halo, electron cloud effects, and beam scraping. Some examples are described below.

The first component to align on the beam line is the emitter surface and diode geometry. Misalignments of these surfaces relative to one another lead to non-uniform focusing of the beam through diode. This generates initial centroid offsets, a radially non-uniform axial velocity distribution, and beam halo. Examples of these effects are shown in Section 5.1.1. Non-uniform axial velocity distributions and beam halo are

common effects caused by even the slightest misalignments $(<1 \mathrm{~mm}$ and $<1 \mathrm{mrad})$ of 


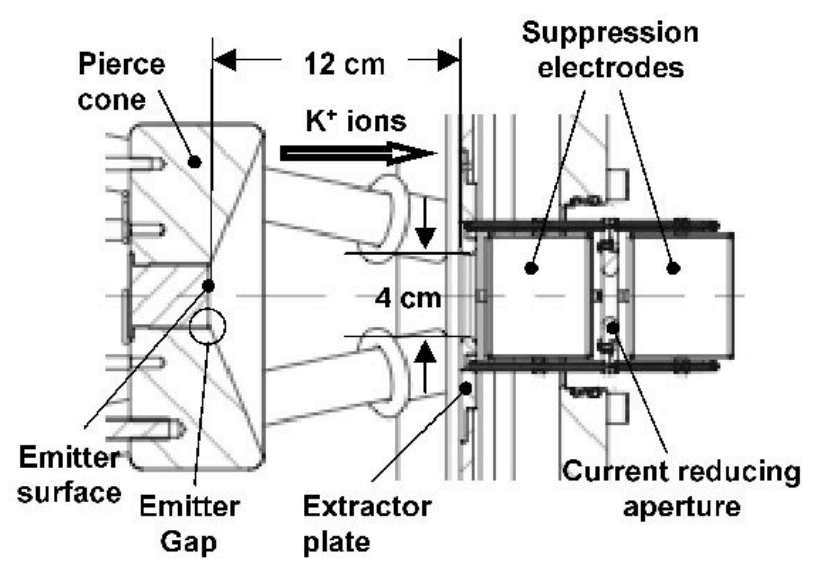

Figure 2.4. Diagram of the Pierce diode geometry and suppression electrodes with removable aperture downstream.

the emitter surface. Improper placement of the emitter surface relative to the Pierce cone (Fig. 2.4) creates a field distortion that accelerates particles at the edge into the beam leading to the sheared or radially contoured distribution. Centroid offsets on the other hand are usually quite small ( $\ll 1 \mathrm{~mm}$ and $\ll 1 \mathrm{mrad}$ ) but are comparable to the misalignments of the emitter if it is misaligned $>1 \mathrm{~mm}$ and $>1 \mathrm{mrad}$.

Misalignments of other components like the focusing elements of a transport lattice also degrade the beam quality. Solenoids are the focusing elements used in the experiment presented in this thesis. Solenoids focus a beam axisymmetrically, if misaligned they displace a beam in all four dimensions of transverse phase space. Precise alignment of the axial magnetic field in a solenoid lattice is critical to the beam dynamics. Slight misalignment of a solenoid in a focusing lattice contributes to centroid offsets and cause the beam centroid to carry out a corkscrew orbit. This motion grows axially along a focusing lattice if each additional solenoid is misaligned [35, 36]. This excitation also leads to emittance growth and halo formation [37]. Once the beam centroid is offset and begins a corkscrew orbit the beam distribution becomes distorted and mismatched. Largely distorted and mismatched distributions develop 


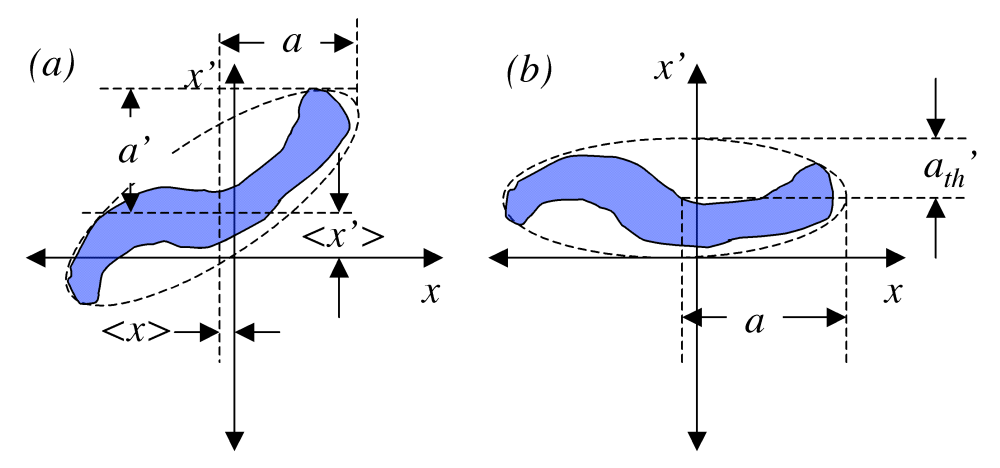

Figure 2.5. (a) Transverse phase space and (b) sheared phase space distributions of a beam with nonlinear focusing effects.

beam halo and increased emittance. These undesired effects have been studied on several electron beam experiments and become catastrophic as the number of misaligned lattice elements increases. Details of these effects are also being studied on NDCX and are explained in Chapter 5.

Below is an example of a transverse phase space distribution which has centroid offsets (Fig. 2.5). The first moments of the distribution $\langle\mathrm{x}\rangle$ and $\left\langle\mathrm{x}^{\prime}\right\rangle$ and the 2rms envelope angle and radius are shown. Comparing Fig. 2.5 with Fig. 2.3 the beam distribution is not a perfect ellipse, it is distorted and the particles are distributed over a larger elliptical area. Considering a 100\% elliptical region for both cases (Fig. 2.3 \& 2.5) and only calculating the particles within the ellipses, the distorted case has a larger calculated emittance. The distortions or hooks at the edges of the distribution may attribute to the misalignment effects discussed above in addition to nonlinear focusing fields.

The fringe components of a solenoid are usually the most significant contributor to nonlinear focusing effects. Consider a finite length current sheet wrapped around the z-axis (in the azimuthal direction). This current sheet acts as an ideal solenoid creating an ideal magnetic field which is uniform except near the ends where the $B_{z}$ 
field is mainly linear versus $\mathrm{r}$. The higher order terms in the fringe are the largest contributors to nonlinear focusing. Further details of these effects measured and simulated on NDCX are in Section 5.3.3.

Another topic that is of particular interest to the general accelerator community is beam degradation, such as emittance growth, due to electron cloud and gas effects [3847]. Mismatched portions of a beam, such as beam halo, photons, and even matched portions of a beam incident upon a material in the path of the particle desorbs gas and electrons [48-57]. The desorbed gas expands into the beam path close to the sound speed and subsequently becomes ionized [58]. In some cases the electron and ionized gas densities are assumed to approach the beam density and significant changes in the charge collected on diagnostics, emittance growth, and fluctuations in the beam envelope are seen. $[12,59]$. Details of the adverse effects of electron clouds and gas on the beam dynamics are presented in Sections 5.3.1 \& 5.4.

\subsection{Longitudinal charged particle beam dynamics}

The remaining two dimensions of phase space yet to be outlined are contained in the longitudinal phase space. In this section we will present the longitudinal beam dynamics relevant to the experiments conducted on NDCX.

\subsubsection{Longitudinal phase space and emittance}

The number of particles in the 2-D component of longitudinal ( $\left.\mathrm{z}-\mathrm{z}^{\prime}\right)$ phase space are shown in the uniform beam distribution in blue (Fig. 2.6). Again we use $z^{\prime}$ to show small differences in the average axial velocity $\left\langle v_{z}\right\rangle$ from head to tail.

$$
z_{i}^{\prime}=\frac{v_{i}}{\left\langle v_{z}\right\rangle}
$$



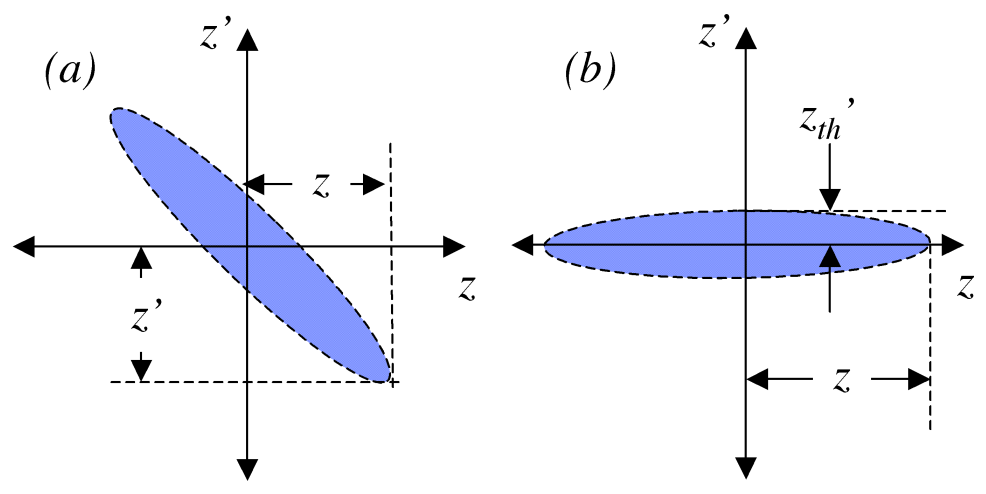

Figure 2.6. Sketch of the (a) Longitudinal phase space; and (b) the sheared distribution with the longitudinal envelope angle ( $\mathrm{z}^{\prime}$ ) removed to show the thermal spread $\mathrm{z}_{t h}^{\prime}$.

where $v_{i}$ is the axial velocity of any particle from the head to the tail of the beam bunch.

An effective area or ellipse is drawn around the distribution to place an upper limit on the calculated emittance. The longitudinal emittance conceptually is identical to the transverse emittance. It is a conserved quantity that is considered to be a measure of the spread in axial velocity. For a beam with no space charge the longitudinal emittance or temperature is what limits an axially compressed bunch length from becoming infinitely thin. Mathematically the longitudinal emittance for a uniform beam is defined as

$$
\epsilon_{z}=\sqrt{\left\langle z^{2}\right\rangle\left\langle z^{2}\right\rangle-\left\langle z z^{\prime}\right\rangle^{2}},
$$

where $\left\langle\mathrm{z}^{2}\right\rangle$ and $\left\langle\mathrm{z}^{2}\right\rangle$ are the second moments of the distribution and the third term underneath the square root is the correlation term which accounts for the expansion and contraction of the beam bunch. If $\left\langle\mathrm{zz}^{\prime}\right\rangle=0$ then the longitudinal emittance is defined as [60]

$$
\epsilon_{z}=z z_{t h}^{\prime},
$$

which is the initial condition at the emitter and $z$ is the longitudinal beam width (or 
bunch half-length) and $z^{\prime}{ }_{t h}$ is the axial thermal width of the beam

$$
\begin{gathered}
v_{t h}^{\prime}=\frac{\Delta v_{z}}{v_{z}}=\frac{v_{T z}}{v_{z}}, \\
\Delta v_{z}=v_{T z}=\sqrt{\frac{T_{z}}{m}},
\end{gathered}
$$

where $\Delta \mathrm{v}_{z}$ is the longitudinal velocity spread, $\mathrm{v}_{T z}$ is the thermal velocity, $T_{z}$ is the longitudinal beam temperature in $\mathrm{eV}$ and $m$ is the mass in $\mathrm{eV} / \mathrm{c}^{2}$.

\section{Longitudinal temperature}

The longitudinal temperature is derived from the assumption that the longitudinal velocity distribution is a 1-D Maxwellian about the average velocity of the beam [61]. The energy spread $\Delta E$ or longitudinal thermal energy $E_{T z}$ of the beam in $\mathrm{eV}$ is shown below

$$
\Delta E=E_{T z}=\frac{1}{2} T_{z}=\frac{1}{2} m v_{T z}^{2}
$$

The kinetic energy of a non-relativistic beam is defined as $E=1 / 2 m v^{2}$. The energy spread $\Delta E$ is related to the longitudinal velocity spread $\Delta v_{z}$ by differentiation

$$
\Delta E=m v \Delta v_{z}
$$

The spread in the energy of the beam $\Delta E$ with respect to the initial kinetic energy $E_{o}$ of the beam can be derived as

$$
\frac{\Delta E}{E_{o}}=\frac{2 \Delta v_{z}}{v_{o}}=\frac{2 v_{T z}}{v_{o}}=\sqrt{\frac{2 T_{z}}{E_{o}}} .
$$

The longitudinal temperature of the beam $T_{z}$ is found from the above equation

$$
T_{z}=\frac{(\Delta E)^{2}}{2 E_{o}}
$$

where $\Delta E$ is the energy spread of a particular slice of the beam in longitudinal phase space. 


\subsubsection{Longitudinal envelope equation}

The longitudinal envelope equation describes the evolution of the longitudinal beam bunch subject to externally applied and beam self fields. The longitudinal envelope equation is derived below from the equation of motion and assuming radially symmetric fields [62]. There are only applied axial electric fields $E_{a}(z)$ and axial elec-

tric fields due to the beam space charge $E_{s}(z)$ in the longitudinal envelope equation, where the subscripts $a$ and $s$ denote the applied and self fields.

$$
Z^{\prime \prime}=-\frac{\gamma^{\prime} Z^{\prime}}{\beta^{2} \gamma}-\left(\frac{q E_{z}^{\prime}}{\beta^{2} \gamma^{3} m c^{2}}\right) Z+\frac{\epsilon_{z}^{2}}{Z^{3}}+\frac{K_{z}}{Z^{2}} .
$$

Like the transverse envelope equation, the longitudinal envelope equation has many terms, the first $Z^{\prime \prime}$ is the second derivative of the bunch width with respect to the axis of propagation or the axial fluctuation of the beam bunch width. The second term is a bunching term due to axial acceleration. The third term is a bunching term due to axial components of applied electric fields.

The fourth term is a defocusing term due to the longitudinal emittance or thermal spread of the beam, where $\epsilon_{z}$ is the rms emittance derived in Section 2.4.1 (Eq. 2.59). For neutralized axial compression on the NDCX and other ion beam drivers this term sets the upper limit for peak axial compression. However we will see below how imperfections in the velocity tilt also limit peak axial compression.

The fifth and final term is a defocusing term due to the longitudinal beam space charge. The longitudinal electric field is derived from Gauss's Law (Eq. 2.40) [62, 63]

$$
E_{z}=\frac{-g}{4 \pi \epsilon_{o}} \frac{\partial \lambda}{\partial z}
$$

where $g$ is the g-factor

$$
g=2 \ln \left(\frac{r_{\text {pipe }}}{a}\right)
$$


where $r_{\text {pipe }}$ is the pipe radius and $a$ is the beam radius. $\partial \lambda / \partial \mathrm{z}$ is the variation of the line charge density along the bunch length which can be approximated by

$$
\frac{\partial \lambda}{\partial z}=-\frac{2 \lambda}{a} \frac{\partial a}{\partial z}
$$

The longitudinal perveance $K_{z}$ in Eq. (2.67) has a unit of length and can be expressed in terms of the generalized perveance $K$.

$$
K_{z}=K g z=\frac{q \lambda z}{2 \pi \epsilon_{o} E} \ln \left(\frac{r_{p i p e}}{a}\right) .
$$

\subsubsection{Acceleration and bunching methods}

\section{Acceleration}

On the NDCX we use two methods of acceleration. The first method used is a pulsed diode. Ions are accelerated from rest at the emitter surface across a $12 \mathrm{~cm}$ gap. The voltage is supplied by a Marx capacitor bank which has a maximum voltage limit of $500 \mathrm{kV}$. The voltage is applied in adjustable pulse lengths which range from a minimum limited by the transit time in the diode $\tau_{D}$ and a maximum of tens of microseconds without the risk of breakdown. $\tau_{D}$ is defined as

$$
\tau_{D}=\frac{3 d}{v_{z}}
$$

where $d$ is the diode gap. The diode gap on NDCX is $12 \mathrm{~cm}$, so for a $300 \mathrm{kV}$ beam, $\tau_{D}$ is $\sim 300$ ns. The repetition rate at which we can pulse the Marx is limited by the charging time of the power supply used, which is 3 seconds. Typically most experiments were carried out using a $300 \mathrm{kV}$ pulse with lengths of 3-10 $\mu$ s at a repetition rate of $\leq 0.1 \mathrm{~Hz}$.

The second method uses a ramped traveling wave to accelerate ions. This concept, also called the Pulse Line Ion Accelerator (PLIA), will not be discussed in this thesis and explanations can be found in Refs.[64-66]. 


\section{Bunching}

Another method of acceleration, induction, can easily be applied on NDCX but has not been tested. Currently we use induction to axially compress charged beam bunches. In order to axially compress a charged beam bunch a head to tail velocity ramp must be applied. The ramp is not necessarily one polarity or the other it is just needs to accelerate the tail particles more than the head particles. In order to minimize high voltage standoff in the gap a bipolar pulse was used. However, for target heating it is advantageous to use a positive unipolar pulse to effectively accelerate the particles while longitudinally compressing them.

Axial compression of space-charge dominated beams in vacuum was examined thoroughly with theory and simulation [67-69]. Longitudinal space charge limits the beam compression ratio $R$, the ratio of the initial-to-final current, to $\leq 10$ and an experiment reported $R \sim 5$ [70]. If the beam space-charge is perfectly neutralized only two factors limit the axial compression, the velocity tilt $\Delta v_{o}$ and the velocity spread $\Delta v_{z}$ of the uncompressed beam bunch. The compression ratio $R$ is defined below

$$
R=\frac{\Delta v_{o}}{\Delta v_{z}} .
$$

The equation for the ideal velocity tilt is derived below. The initial axial velocity of a beam is given as $v_{z o}$, the initial bunch length is given as $t_{o}$, and the longitudinal focal length is given as $f$. The velocity of the leading edge of the compressing beam, $v_{o}$ is defined below.

$$
v_{o}=v_{z o}+\frac{f}{t_{o}}-\left(v_{z o}^{2}+\left(\frac{f}{t_{o}}\right)^{2}\right) .
$$

The velocity tilt, $v_{f}(t)$, applied as a function of time is

$$
v_{f}(t)=\frac{v_{o} f}{f-v_{o} t} .
$$

The applied voltage necessary for the velocity tilt $V(t)$ is given below where $m$ is the 
mass of an ion species in $\mathrm{V} / \mathrm{c}^{2}$.

$$
V(t)=\frac{1}{2} m\left(v_{f}^{2}(t)-v_{z, o}^{2}\right)
$$

The Induction Bunching Module and the plasma sources used for axial compression measurements are explained in detail in Chapter 6.

\subsubsection{Adverse effects on the longitudinal beam dynamics}

All of the equations derived up to this point for the longitudinal beam dynamics assumed an ideal cylindrical beam bunch. Results presented in later chapters lack this ideal scenario, which can help explain the minimum bunch lengths achieved (which are greater than ideal) for axial compression measurements and longitudinal temperatures measured. There are several factors that can increase the longitudinal temperature and pulse widths measured. They include the diode voltage and the velocity tilt quality.

First, the Marx voltage waveform that we use to extract and initially accelerate the beam through the diode is not a perfect square pulse (Fig. 2.7). There is a finite rise time $\left(>\tau_{D}\right)$ in the waveform and there are voltage oscillations. Before reaching the peak voltage, the voltage of beam has an increasing slope at the head of the beam (from 300-800 ns). After 800 ns the pulsed waveform has a decreasing slope. These variations in voltage lead to overtaking and bunching of the beam.

Voltage oscillations in the diode also add a velocity tilt to the beam. However, these oscillations must occur over a very narrow range of frequencies. The criteria is set by the acceleration time across the diode $t_{a}$ and the bunch duration being considered $t_{b}$. The period $T$ of the voltage oscillation must be $t_{a}<T<t_{b}$. On NDCX $t_{a}$ is typically $100 \mathrm{~ns}$ for a $300 \mathrm{keV}$ across the $12 \mathrm{~cm}$ diode. This means frequencies $<10 \mathrm{MHz}$ can add a velocity tilt to a beam bunch $>100 \mathrm{~ns}$ in duration. 


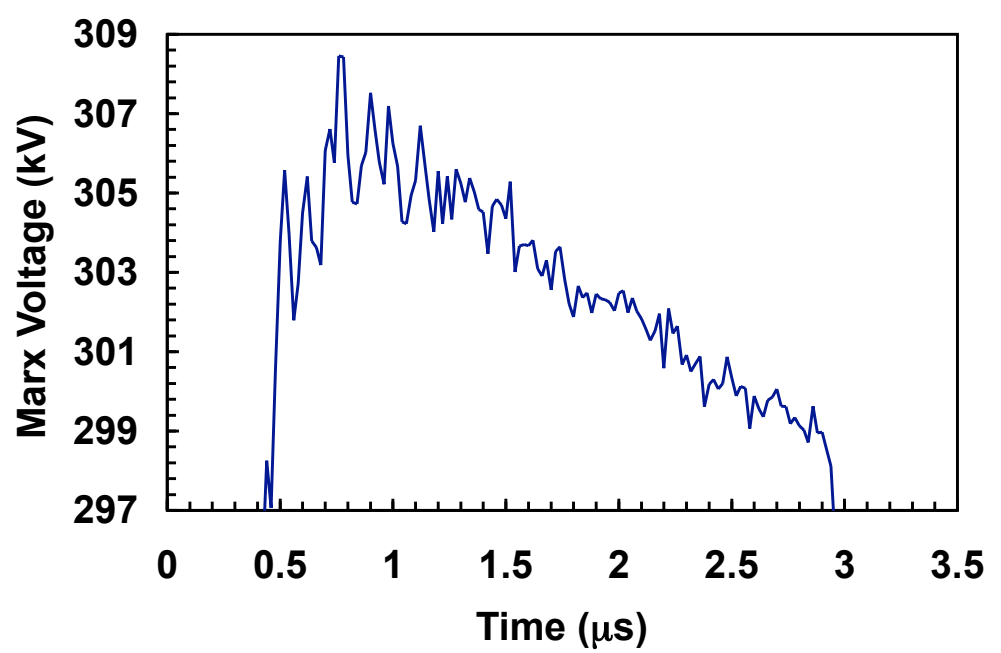

Figure 2.7. Example of a Marx voltage waveform used to accelerate ions through the diode in Fig. 2.4.

There are two large frequency components embedded in the Marx waveform, 1 and 6 $\mathrm{MHz}$, which add a velocity tilt to beam (Fig. 2.7).

Next, an imperfect velocity tilt reduces the expected axial compression from an ideal tilt. The induction cell used only provides a finite amount of the volt seconds specified by the ideal waveform due to hardware limitations. Also the induction gap about which the velocity tilt is applied has a finite width of $3 \mathrm{~cm}$. This induces two effects to the longitudinal dynamics a transit time effect and fringe field effects. The transit time of a $300-\mathrm{keV} \mathrm{K}^{+}$ion across the induction gap is about $25 \mathrm{~ns}$. As any of the ion cross the gap they see a range of voltages, instead of one prescribed voltage for peak compression. This reduces the maximum achievable axial compression but does not increase the longitudinal temperature of the beam.

Fringe fields also effect the velocity tilt. The fields at the edges of the gap add nonlinear focusing terms to the ions as they enter and exit the induction gap. This reduces the uniformity of the beam adding larger pulse widths and possibly a pedestal 
at the base of the peak compressed pulse. Similar effects are caused by voltage oscillations in velocity tilt waveform.

\subsection{Coupled longitudinal and transverse beam dy- namics}

There are also effects due to coupled transverse and longitudinal beam dynamics. The most prominent effect occurs when axially bunching the beam. The induction gap used to axially compress the beam bunch produces radial electric fields $E_{r}$ in addition to the axial electric fields $E_{z}$. The beam envelope receives a net positive radial impulse

on the upstream side of the gap (assuming a positive voltage difference $V(t)$ across the gap) and net negative radial impulse on the downstream side of the gap.

On the NDCX the gap voltage changes significantly during the 25-ns transit time of the $\mathrm{K}^{+}$ions through the lens. As a result the net radial forces upstream and downstream of the center of the gap do not cancel since $E_{r}$ is proportional to the time dependent voltage $V(t)$. The large and negative $d V / d t$, which is required for longitudinal focusing, has a net transverse defocusing effect. In the thin-lens approximation the radial forces are modeled by a delta function separated by the width of the gap, $d$. The time dependent change in radial velocity of a non-relativistic ion going through the gap $\Delta v_{r}(t)$ is approximated by the formula [71]

$$
\Delta v_{r}(t) \approx-\frac{e r}{2 m v_{z o} d}\left(\Delta V\left(t_{o}\right)-\Delta V\left(t_{o}-\frac{d}{v_{z o}}\right)\right) \approx-\frac{e r}{2 m v_{z o}^{2}} \frac{d V(t)}{d t},
$$

where $v_{z o}$ is the initial axial velocity of the ions upstream of the gap. The time dependent change in the axial velocity $\Delta v_{z}(t)$ due to the time dependent voltage 
$V(t)$ is given by

$$
\Delta v_{z}(t)=v_{z o}-\sqrt{v_{z o}^{2}-\frac{2 V(t)}{m v_{z o}^{2}}} .
$$

The total effect on the beam envelope neglecting the change in radius across the gap is given by

$$
a^{\prime}(t)=\frac{v_{r o}+\Delta v_{r}(t)}{v_{z o}+\Delta v_{z}(t)}
$$

where $a^{\prime}$ is the $2 \mathrm{rms}$ angle at the exit of the gap and $v_{r o}$ is the initial radial velocity of the ions upstream of the gap. The expected increase in radius of the focused distribution due to chromatic aberrations is shown in detail for different focusing geometries in Section 6.4. 


\section{Chapter 3}

\section{Diagnostics, data acquisition and analysis}

In this chapter we will discuss the different diagnostic techniques used to measure the beam parameters on NDCX.

\subsection{Beam current}

There are several methods of measuring the beam current; we will only address those used on the NDCX. The measured beam current provides a quick assessment of the functionality of the beam injection, matching, and transport. If the beam current is lower or higher than is expected there can be a number of problems. Generally the current is not higher unless the emitter is operated below the space charge limit and is overfocused by the diode fields through the current reducing aperture (Fig. 1.9). If the current is lower than expected, then the beam could be scraping due to a number of causes. Three common causes of beam scraping are: a focusing element may not be operating properly, the lattice tune is incorrect, or, again, operating the emitter 


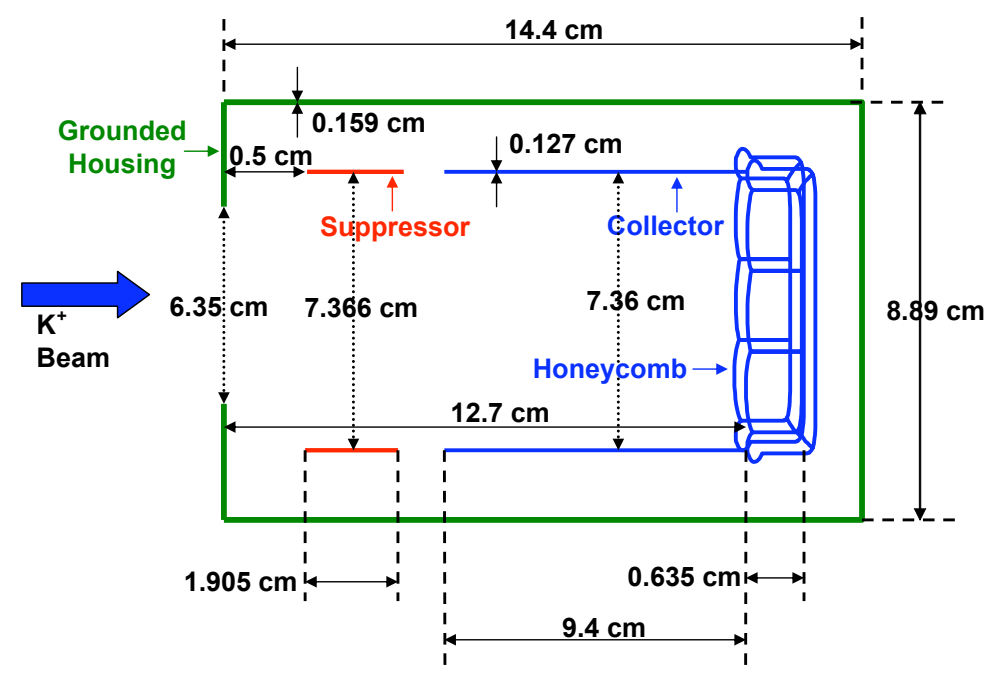

Figure 3.1. Diagram of the standard Faraday cup used to measure the beam current at the exit of the diode and different transport configurations on the NDCX. All electrodes and the housing have cylindrical symmetry.

short of the space charge limit. An emitter failure causes unforeseen consequences to the beam current and beam distribution.

\subsubsection{Faraday cup}

There are two different Faraday cups used on the NDCX. The first shown in Fig. 3.1 is a standard Faraday cup. It consists of two electrodes. The first is the short upstream cylindrical suppression electrode. The second is the long cylindrical can or collector electrode. Both electrodes are electrically isolated from one another and the grounded housing in which they are encased. There is also a honeycomb or gridded surface at the base of the collector that is electrically connected to the collector to reduce the beam fields and electron cloud and gas effects at the base of the collector.

The bias configuration of the cup is as follows: the suppressor is biased negatively to suppress electrons emitted from the collector surface, from backstreaming into the 


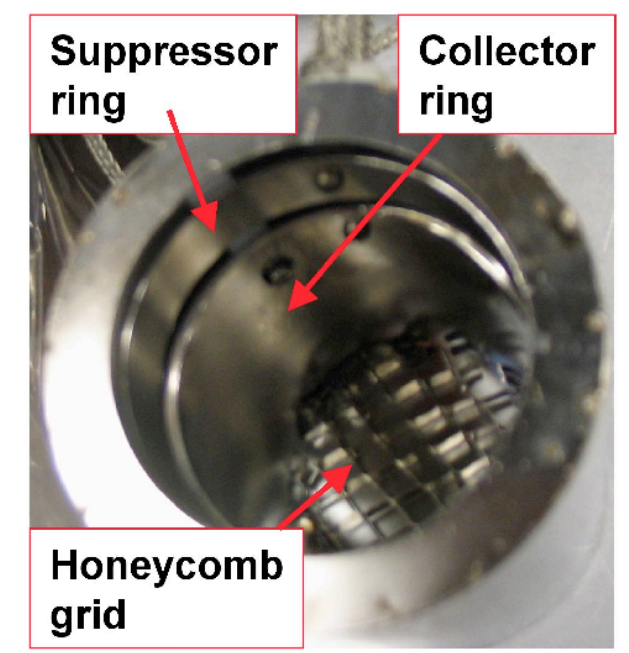

Figure 3.2. Photograph of standard Faraday cup used on the NDCX with electrodes labeled.

beam. The suppressor also rejects low energy electrons that may be traveling with the beam from entering the cup. The collector is biased positively to measure the positive ion current and help prevent secondary electrons from leaving the collector. When the beam is normally incident upon a flat surface it desorbs gas and makes secondary electrons. This gas migrates away from the surface and can be further ionized making additional electrons and gas ions. Having the gridded honeycomb surface reduces the local field preventing fast migration of electron-ion pairs to the collector and suppressor.

There is a signal which can be measured on the suppressor and collector through a capacitive coupling which is applied to each of them individually (Fig. 3.3). High voltage is applied by a power supply (HV from PS) to the electrode (HV to Electrode). The capacitive coupling allows one to monitor the voltage drop across the resistor (R3). This voltage drop can easily by used to determine the charge or current collected on the diagnostic by Ohm's Law $(\mathrm{V}=\mathrm{IR}) .50 \Omega$ is chosen for $\mathrm{R}$ to match the characteristic impedance of the circuit and avoid signal reflections. 


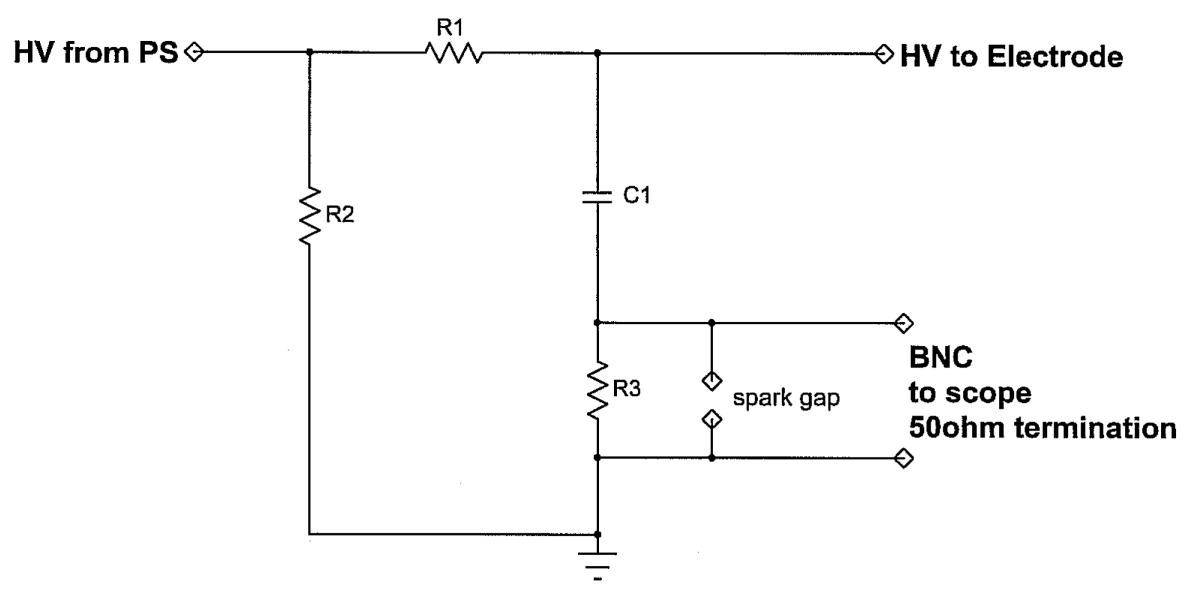

Figure 3.3. Schematic of the capacitive coupling circuit used to monitor the signals on the suppressor and collector electrodes of the Faraday cup. High voltage is applied through a power supply (HV from PS) to the electrode ( $\mathrm{HV}$ to Electrode). The capacitive coupling allows one to monitor the voltage drop across the resistor (R3).

The signals measured at the exit of the diode (Fig. 1.9) and downstream of the four-solenoid transport lattice (Fig. 1.11) are shown in Fig. 3.4. The beam induces an image charge on the suppressor electrode and a positive capacitive image current is measured on the suppressor as the beam enters the electrode and a negative capacitive image current is measured on the suppressor as it exits the electrode. These capacitive signals are proportional to the derivative of the beam current measured on the collector. The signals in Fig. 3.4(b) are displaced in time due to the time of flight over $2.6 \mathrm{~m}$. A peak is also observed at the beginning of the current waveform on the collector in Fig. 3.4(b) due to overtaking in the beam head.

\subsubsection{Fast pinhole Faraday cup}

Experiments to focus transversely and simultaneously bunch a space charge dominated ion beam required a Faraday cup much different than the conventional one described above. In order to maximize the beam density or intensity, the space charge 

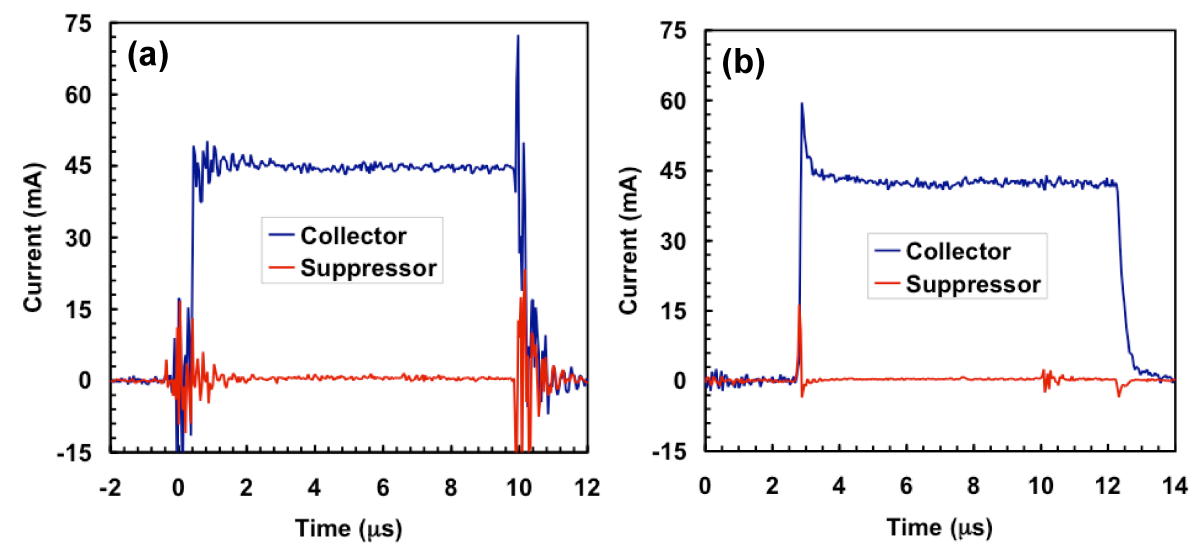

Figure 3.4. Measured beam current on the collector (blue) and image current on the suppressor (red) (a) $28 \mathrm{~cm}$ downstream of the diode $(\mathrm{z}=40 \mathrm{~cm}$ ) and; (b) $54 \mathrm{~cm}$ downstream of the exit the four-solenoid transport lattice $(\mathrm{z}=304 \mathrm{~cm})$.

of the beam must be neutralized by a cold plasma of equal or greater density. This neutralizing plasma presents difficulty in measuring the beam current due to plasma shielding of the electrodes (see Section 2.2). There are two additional drawbacks to the standard Faraday cup: the capacitance of the diagnostic and the transit time of ions in the diagnostic. The capacitance limits the response time of the diagnostic to $>10 \mathrm{~ns}$ and the transit time of ions in the diagnostic is $\sim 100 \mathrm{~ns}$. A fully neutralized ion beam with a longitudinal temperature $\mathrm{T}_{\mathrm{z}}<1 \mathrm{eV}$ can be axially compressed to pulse widths $\mathrm{t}_{\mathrm{b}} \leq 2 \mathrm{~ns}$ for an ideal velocity tilt applied to a $200 \mathrm{~ns}$ bunch with a focal length of $\sim 1 \mathrm{~m}$.

A pinhole Faraday cup capable of screening out unwanted plasma electrons and ions and yielding response times $\leq 1 \mathrm{~ns}$ is desired for these measurements. The first pinhole Faraday cup was designed for a plasma environment with a density of $10^{10}$ $\mathrm{cm}^{-3}$ and a beam spot size of $\geq 1 \mathrm{~cm}[72]$. This pinhole Faraday cup has two hole plates and a collector [Fig. 3.5(a)]. Two hole plates were used to screen out plasma electrons and ions from confounding the measurement of the beam current. The first hole plate had holes with radii nearly equal to the Debye length of the plasma ( 


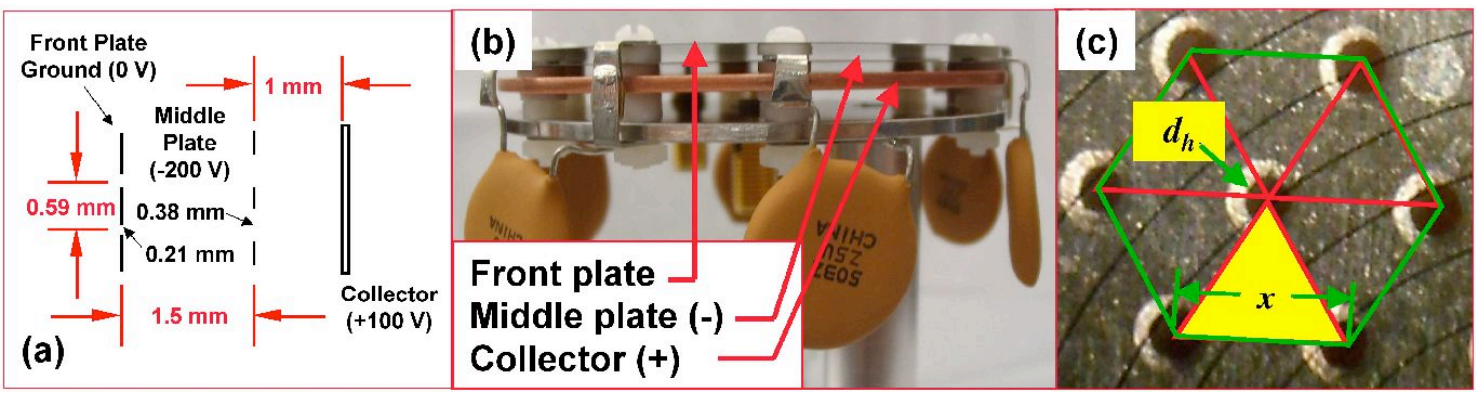

Figure 3.5. Schematic of the (a) pinhole Faraday cup geometry; (b) photograph of the constructed pinhole Faraday cup and; (c) close-up of the pinhole pattern.

$0.1 \mathrm{~mm})$. The second hole plate, which acts as a suppressor of electrons, has holes nearly a factor of 2 larger to allow an expanding and possibly misaligned beam to pass though without scraping. Although this was a method for screening out plasma electrons and ions, it also cut down the acceptance level of beam ions that reach the collector by at least a geometric factor $f$, the ratio of the pinhole pattern area $A_{\Delta}$ to the pinhole area $A_{h}$.

$$
f=\frac{A_{\Delta}}{A_{h}}=\frac{\frac{\sqrt{3}}{2} x^{2}}{\frac{\pi}{4} d_{h}^{2}},
$$

where the measured hole diameter $d_{h}=210 \mu \mathrm{m}$ and the hole to hole spacing $x=590$ $\mu m$ [Fig. 3.5(c)]. So the maximum amount of measured current will be reduced by $f$ $=8.7$. However, electron cloud and gas effects have an effect which further decreases the net current measured on the collector. This will be shown in Section 6.2.7.

After screening out unwanted plasma electrons and ions the next requirement is to minimize the response time of the collector signal and the compressed pulse width measured. The response time of the collector signal is limited by three constraints. The first described is the RC time constant, which is a function of the geometry of the cup. The capacitance of the cup is

$$
C=\frac{\epsilon_{o} A}{\delta z}
$$


The area of the cup, $A$, with $6.35 \mathrm{~cm}$ diameter plates is $31.67 \mathrm{~cm}^{2}$ and the spacing from the middle plate to the collector, $\delta z$, is $1 \mathrm{~mm}$ yielding an estimate of $28 \mathrm{pC}$ for the capacitance. A $50 \Omega$ transmission line provides an RC time constant of $1.4 \mathrm{~ns}$.

Second, the response time can also be limited by the inductance of the capacitive coupling used to monitor the current signal on the diagnostic. Ten low inductance $(<0.1 \mathrm{nH})$ capacitors in parallel were used for $\mathrm{C} 1$ in Fig. 3.3 of the coupling circuit so the lower bound on the minimum resolvable pulse width on the pinhole Faraday cup was set by the RC time constant.

Third, the rise time of the signal on the collector is limited by the transit time of the ions between the two plates. The beam velocity of a $300-\mathrm{keV} \mathrm{K}^{+}$ion is 1.22 $\mathrm{mm} / \mathrm{ns}$ so a $\delta z$ of $1 \mathrm{~mm}$ was used.

Once the diagnostic was designed a compressed pulse duration and compression ratio is also extracted from this measurement. The analysis of this data is quite complex and requires a careful analysis (see Section 6.2). When examining the signals from this diagnostic it is worth noting the typical background and signal to noise levels (Fig. 3.6). The background or bias level of the diagnostic varies in the range of -6 $\pm 6 \mathrm{mV}$. The electrical noise in the diagnostic which is several $\mathrm{MHz}$ in frequency is $\pm 2 \mathrm{mV}$. After performing a background subtraction the signal amplitude with no current density compression is $\geq 25 \mathrm{mV}$ and with current density compression can be as high as $3.0 \mathrm{~V}$.

There are ways to further reduce the response time of the diagnostic. To avoid reflections the diagnostic must continue to be terminated with a matched impedance of $50 \Omega$. However, the capacitance can be reduced further in two ways. First the area of the plates can be reduced to the maximum expected radial excursion of the beam halo of the unfocused beam $\sim 1 \mathrm{~cm}$. Including potential centroid offsets of $\pm 5 \mathrm{~mm}$, 

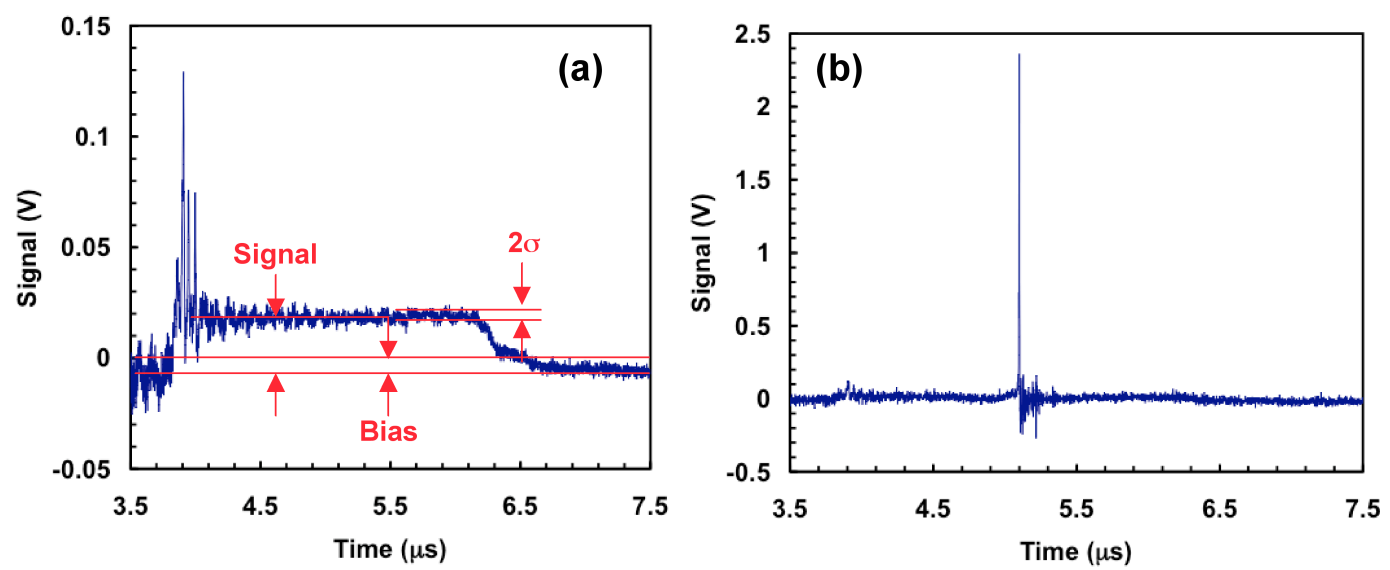

Figure 3.6. Measured signal collected by the fast pinhole Faraday cup for a beam (a) with no current density compression and; (b) with current density compression.

the maximum hole pattern should be no larger than $3 \mathrm{~cm}$ in diameter yielding a plate diameter of $4 \mathrm{~cm}$ and an area of $12.57 \mathrm{~cm}^{2}$. The distance between the plates can be increased from $1 \mathrm{~mm}$ to reduce the capacitance. However, this is not recommended based on the $1.22 \mathrm{~mm} / \mathrm{ns}$ transit time suggested above. These improvements have been suggested for the next generation design of a fast pinhole Faraday cup.

\subsubsection{Optical Faraday cup}

A scintillator and a phototube were used to make optical measurements of the beam current profile. The scintillator material we use is a $100-\mu \mathrm{m}$ thick alumina $\left(96 \% \mathrm{Al}_{2} \mathrm{O}_{3}\right)$ wafer [73].

\section{Scintillator}

A scintillator is a material that emits light, or scintillates, due to atomic excitations when absorbing electromagnetic or charged particle radiation. The light provided by the scintillator has a characteristic rise time followed by an exponential

decrease. In our case a particle enters the material and collides with atomic electrons, 
exciting them to higher energy levels; this is the rise time. After a very short period of time the electrons decay to their ground states, causing emission of light; this is called the $1 / \mathrm{e}$ fall time or decay time. For our case a short decay time $(<1 \mathrm{~ns})$ is desired. The decay time is the relevant response time because it is the time between the arrival of radiation in the detector and the photons emitted.

If we assume that the rate of decay is proportional to the number of excited atoms, the number of emitted photons per time unit, $N$, is:

$$
N=N_{o} e^{-\frac{t}{\tau}},
$$

where $N_{o}$ is the total number of emitted photons, $t$ is time unit, and $\tau$ is the decay constant, which is specific to a certain scintillator material.

Impact from beam ions cause the scintillator surface to accumulate charge and possibly arc over since it is an insulator. We place a transparent wire mesh or hole plate [74] about 100 mils upstream of the scintillator to provide secondary electrons to charge-neutralize the insulating scintillator surface. When beam ions strike the transparent mesh approximately 10-100 secondary electrons are made per ion, depending on the angle of incidence with the mesh surface [50, 55-57], which sufficiently neutralizes the scintillator surface to avoid any arcing.

\section{Phototube}

A Hamamatsu R1194U series biplanar phototube [75] was used to measure the beam-induced light emission from the scintillator. This phototube has a spectral response from 300-1100 $\mathrm{nm}$ which is applicable for ranges from vacuum UV to infrared light in the electromagnetic spectrum. This series of phototube has an ultra-fast photodetector with a response time of $270 \mathrm{ps}$ and a $100 \mathrm{ps}$ fall time.

Due to the phototube's sensitivity to ambient light, a pulsed Displaytech Ferro- 

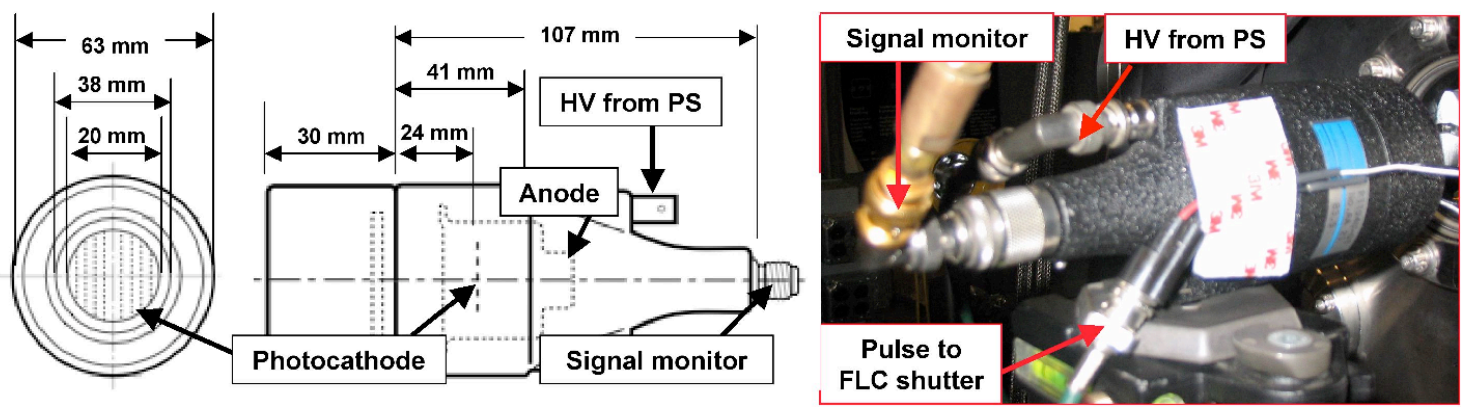

Figure 3.7. Schematic layout of the Hamamatsu R1194U series biplanar phototube.

electric Liquid Crystal (FLC) shutter [76] was gated by the beam triggering system to expose the phototube for $>10 \mu \mathrm{s}$, which was more than a sufficient amount of time to monitor the beam-induced light emission from the scintillator. This method was used to measure the uncompressed and compressed light emission. However, the distance from the light source (the scintillator) to the phototube was no less than 30 cm because of the vacuum tank design. As a result low light collection efficiency and signal-to-noise ratio $(\mathrm{S} / \mathrm{N})$ made it difficult to extract a compression ratio [Section 2.4 .3 \& Eq. (2.73)].

When examining the signals from this diagnostic it is worth noting the typical background and signal to noise levels (Fig. 3.8). The background or bias level of the diagnostic due to background light is $>10 \mathrm{mV}$. The electrical noise in the diagnostic which is several $\mathrm{MHz}$ in frequency is $\pm 2 \mathrm{mV}$. After performing a background subtraction the signal amplitude with no current density compression is about $7 \mathrm{mV}$ and with current density compression can be $>300 \mathrm{mV}$. This demonstrates the lack of $\mathrm{S} / \mathrm{N}$ in the diagnostic, especially for measurements without current density compression, making it difficult to determine a compression ratio.

Measurements were made to understand the phototube's response to the spatial (transverse and longitudinal) location of a light source. The light source used was a 5- 

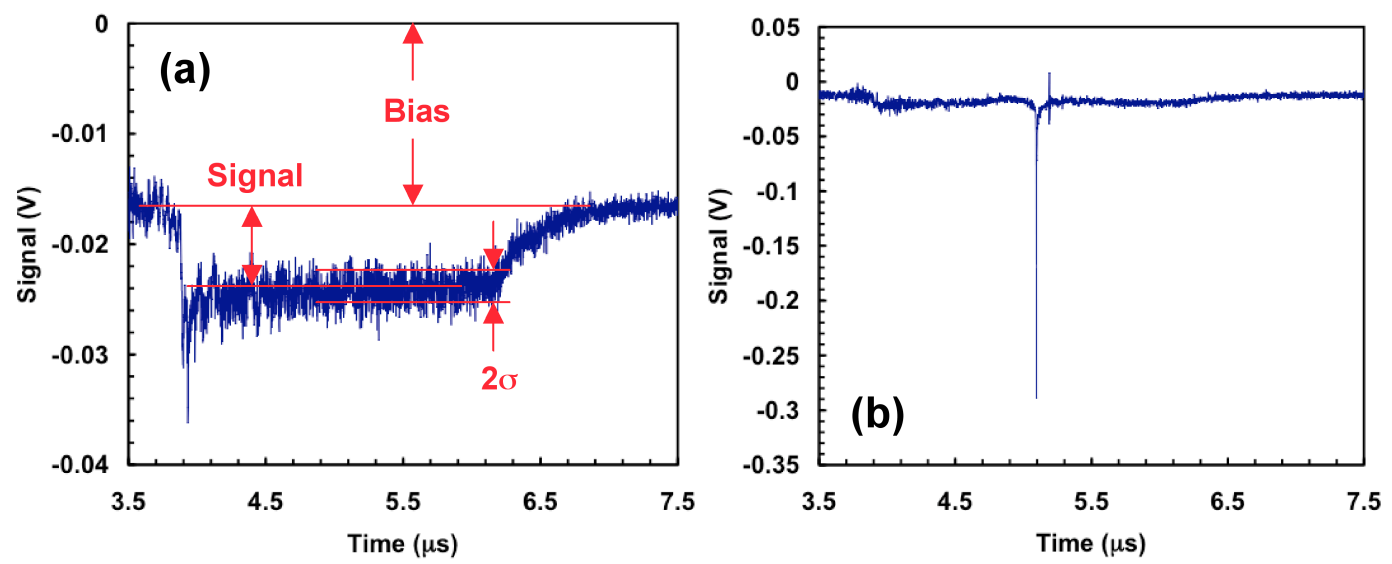

Figure 3.8. Measured signal of light collected by the Hamamatsu R1194U phototube for a beam (a) with no current density compression and; (b) with current density compression.

$\mu$ s pulsed light emitting diode (LED). The measurements showed a strong dependence on the location of the light source. A measurement of the transverse dependence with the LED axially displaced $10 \mathrm{~cm}$ from the phototube shows a linear fall-off in the integrated light collected once the LED is $5 \mathrm{~mm}$ off-axis [Fig. 3.9(a)]. A separate measurement was also made for the longitudinal dependence with the LED aligned transversely. Fig. 3.9(b) shows the integrated light collected by the phototube falls off approximately as the square of the displacement. Both of these measurements display the sensitivity in the phototube's detection capability and warrant the use of focusing lenses or a fiber optic array to increase the light detected by the phototube at distances comparable to the distance from the scintillator to phototube $(\sim 30 \mathrm{~cm})$ in future experiments.

Documentation of the signal to voltage characteristics of the phototube are not available from the manufacturer. So an experiment was conducted to characterize the light collection vs. voltage applied and the dependence was found to be linear as expected. 

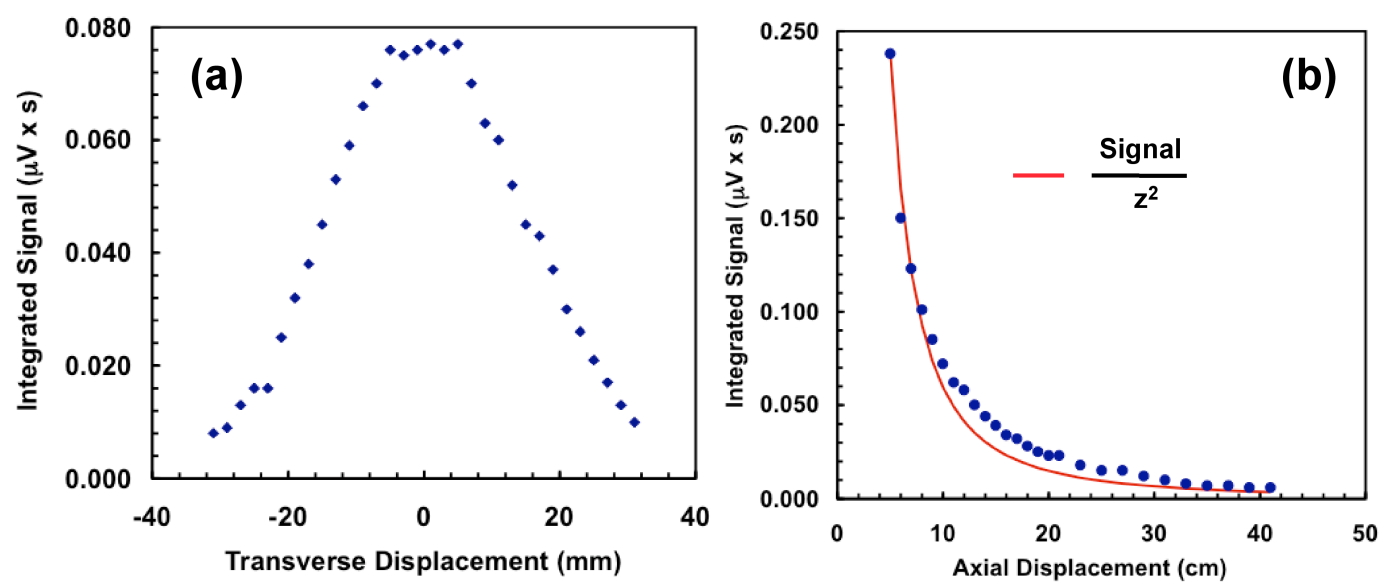

Figure 3.9. Measurement of the spatial dependence in the integrated light collected by the Hamamatsu R1194U phototube (a) transversely with the LED displaced axially by $10 \mathrm{~cm}$ and; (b) longitudinally with the LED aligned transversely.

\subsection{Transverse beam dynamics}

Transverse beam dynamics measurements indicate the rms envelope parameters and more detailed information about non-linear focusing field abberations, misalignments, and space charge waves. Since these measurements intercept most of the beam, in some cases they induce false fluctuations due to beam-induced electron cloud and gas effects (see Chap 5).

\subsubsection{Beam current density and profile}

The beam current density $[\mathrm{J}(\mathrm{x}, \mathrm{y})]$ is measured with a $100-\mu \mathrm{m}$ thick alumina $(96 \%$ $\left.\mathrm{Al}_{2} \mathrm{O}_{3}\right)$ scintillator [77]. This is the same material as described above in Section 3.1.3 [73]. The beam-induced light emission from the scintillator is captured with a Princeton Instruments image-intensified gated-CCD camera [78]. This camera has a temporal resolution of $1 \mathrm{~ns}$ and a $512 \times 512 \mathrm{CCD}$ pixel array. Each pixel is $200 \times 200$ $\mu \mathrm{m}$ making the CCD $1 \mathrm{~cm}$ in size. The spatial resolution of the camera is flexible and 
depends on the optical setup. Typically, a lens configuration is used to maximize the size of the beam distribution on the CCD with spatial resolutions $<0.1 \mathrm{~mm} /$ pixel.

This measurement is integrated over $\mathrm{x}$ or $\mathrm{y}$ to provide a vertical or horizontal profile of the beam distribution. The transverse beam profile indicates the uniformity and alignment of the beam distribution over $\mathrm{x}$ or $\mathrm{y}$. The alignment can be determined from a premeasured fiducial on the scintillator frame. From this fiducial, the center of the CCD image relative to the beam centerline can be determined and the first moments of the beam distribution $(\langle\mathrm{x}\rangle,\langle\mathrm{y}\rangle)$ can be extracted. These optical measurements are collected with the camera downstream of the scintillator if the diagnostic is located near the end of the accelerator.

However, if the accelerator has the diagnostic a few meters upstream from the end of the accelerator, the light collection efficiency to the end of the accelerator is reduced. Therefore an optical diagnostic that can view the scintillator perpendicular to the beam axis is necessary. This diagnostic includes the same beam intercepting $\mathrm{Al}_{2} \mathrm{O}_{3}$ scintillator, a front side mirror placed downstream of the scintillator $45^{\circ}$ to the beam axis and scintillator surface, and an image intensified gated- CCD camera placed perpendicular to the beam axis (Fig. 3.10). This makes it possible to measure all the transverse beam dynamics perpendicular to the beam axis with excellent light collection efficiency and high resolution.

The transverse beam profile is also measured using a single slit and Faraday collector or slit-cup (Fig. 3.11). A slit-cup on NDCX consists of a grounded $0.1 \mathrm{~mm}$ $\times 57 \mathrm{~mm}$ slit followed downstream by a wire mesh and larger slit plate which is biased negatively or positively depending on the operating conditions. Just downstream of the larger slit plate is the collector which is also biased negatively or positively. The transverse profiles were measured on NDCX by using a vertically oriented slit-cup driven in the horizontal direction to measure the horizontal (x) profile and similarly 


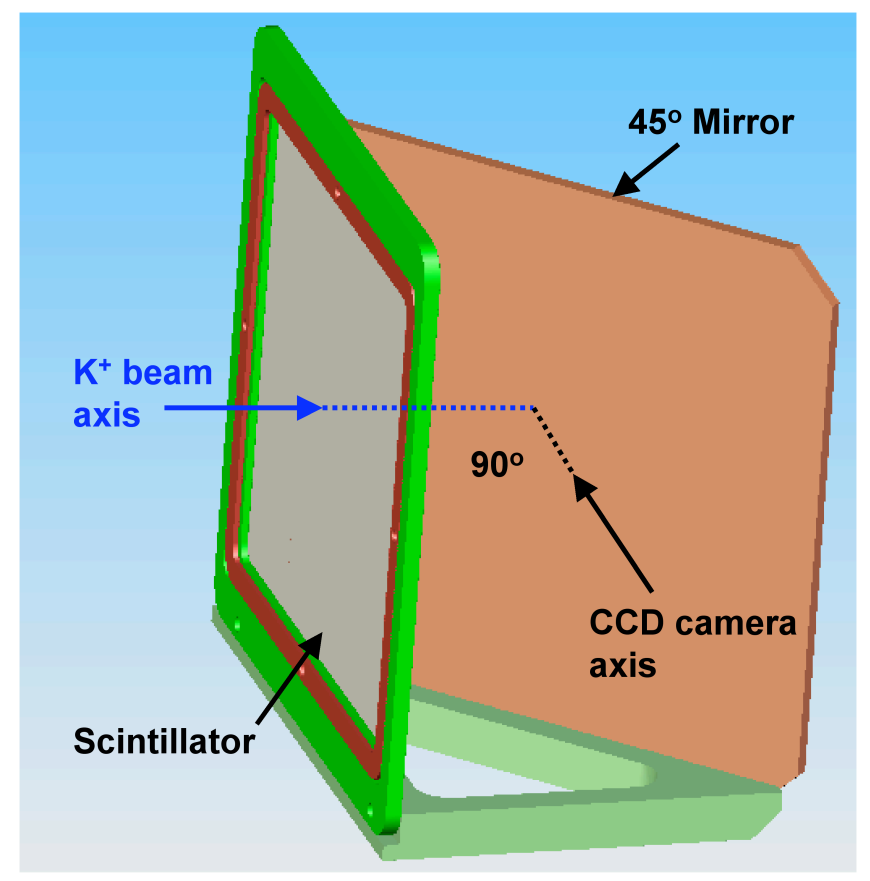

Figure 3.10. Sketch of the optical diagnostic.

for the vertical (y) profile. For operating conditions where we wanted to measure the approximate ion current of the beam, the collector is biased positively to collect the ion current and the mesh upstream is biased negatively to suppress electrons. Typical current levels range from $1 \leq \mathrm{I}(\mathrm{mA}) \leq 0.05$ depending on the current density with $\mathrm{S} / \mathrm{N}$ ratio $>10$.

In conditions where we wanted to increase the $\mathrm{S} / \mathrm{N}$ (i.e. lower current densities) we biased the collector negatively and the upstream mesh positively to measure the secondary electrons leaving the collector. Most of our diagnostics are made of stainless steel plates, calculations and measurements show that $\sim 10$ electrons (Section 5.5.2) are made per $\mathrm{K}^{+}$ion at normal incidence with an energy of $300 \mathrm{keV}$ and current density of $170 \mathrm{~A} / \mathrm{m}^{2}$. An amplifier could is also used for cases with lower current density. 


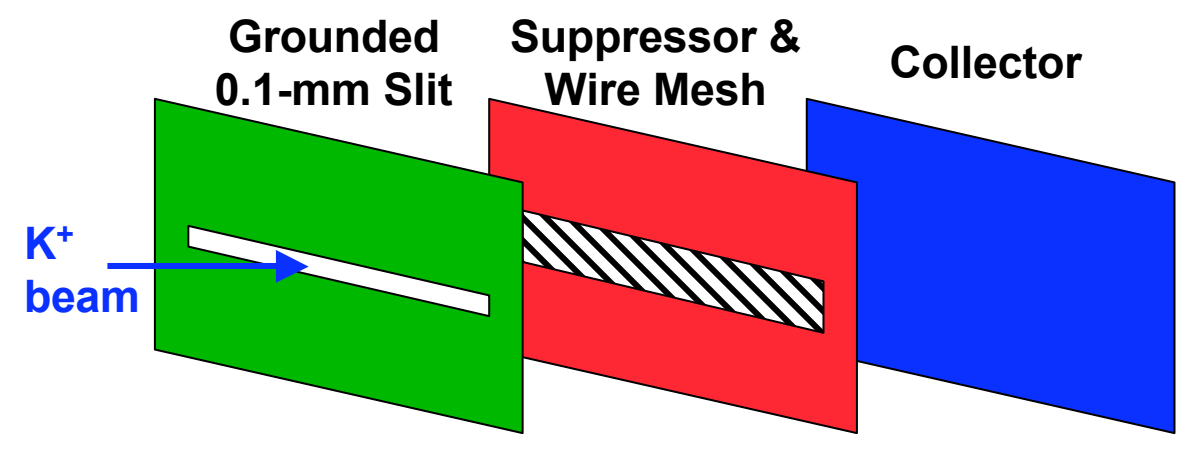

Figure 3.11. Sketch of the slit-cup diagnostic.

\subsubsection{Transverse phase-space and emittance}

Transverse phase space measurements were made using two separate diagnostic techniques. One method used an upstream slit and a downstream slit and a Faraday collector (slit-cup) separated by $\sim 10 \mathrm{~cm}$. The slit-cup used was the same as described above and the upstream slit, which could be moved independently, also had dimensions of $0.1 \mathrm{~mm} \times 57 \mathrm{~mm}$. Step sizes for these measurements were typically 1 $\mathrm{mm} \times 1 \mathrm{mrad}$. The slit-cup generally measured tens of millivolts ( $\sim \mu \mathrm{A}$ of current) and the $\mathrm{S} / \mathrm{N}$ was close to 30 when configured to measure secondary electrons leaving the collector in addition to using an amplifier.

The measured distribution shown in Fig. 3.12 was made using this technique. The 4rms emittance, $\epsilon_{4 \mathrm{rms}}$, envelope parameters, a, a', and centroid offsets $\langle\mathrm{x}\rangle,\left\langle\mathrm{x}^{\prime}\right\rangle$ are all calculated from the distribution. Since the current signals are recorded over the whole beam pulse the time dependence of the beam parameters is also measured [Fig. 3.12(b)].

The disadvantage of this method is the limits of the phase space must be empirically identified before making the measurement in order to avoid missing any of the beam distribution. This is quite a laborious process for a beam with unknown cen- 

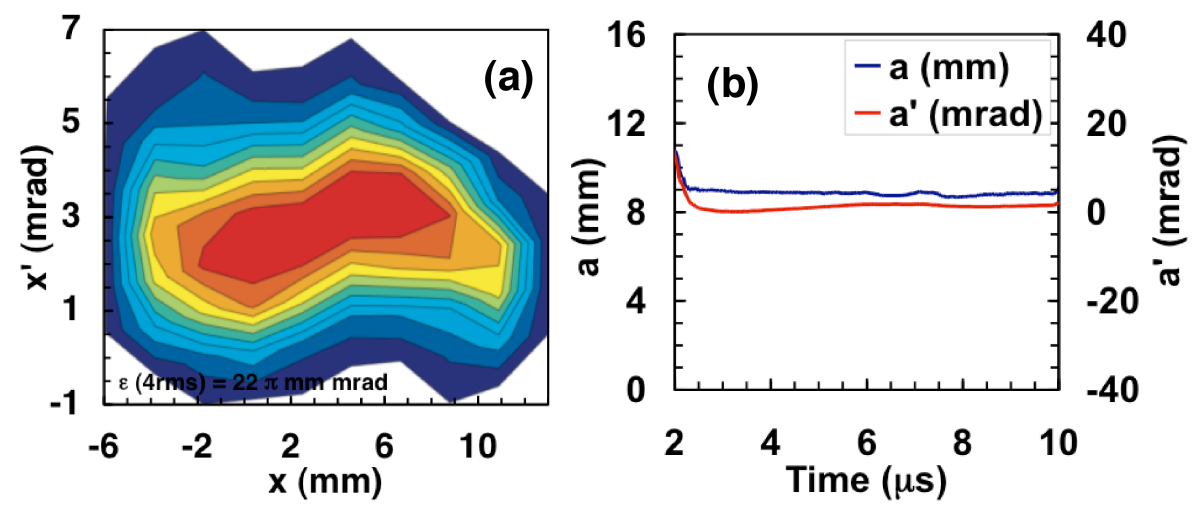

Figure 3.12. (a) Sheared phase space distribution integrated over a 500 ns gate in the middle of the $10 \mu$ s beam pulse; (b) beam envelope as a function of time.

troid offsets and orientation. Also once the measurement begins it can be quite time consuming depending on how fine the experimenter wishes to make the measurement. The typical $1 \mathrm{~mm} \times 1 \mathrm{mrad}$ step size would require 310 individual shots for a beam that fits inside a phase space region $30 \mathrm{~mm} \times 10 \mathrm{mrad}$. At a repetition rate of 0.05 $\mathrm{Hz}$ this would take $>100$ minutes to measure.

The second method used the alumina scintillator mentioned above and an imageintensified gated-CCD camera that imaged beam-induced light emission after masking the beam with a single upstream slit [77]. With the optical collector this method only requires the experimenter to move the upstream slit rather than mapping out all of the angles downstream with a slit-cup, making it much more time efficient. The measured distribution shown in Fig. 3.13 was made using this second technique. The same beam parameters $4 \mathrm{rms}$ emittance, $\epsilon_{4 \mathrm{rms}}$, envelope parameters, a, a', and centroid offsets $\langle\mathrm{x}\rangle,\left\langle\mathrm{x}^{\prime}\right\rangle$ are also calculated from the distribution measured. The disadvantage of this method is that it only provides the beam parameters for the particular time gate chosen. In the middle of the pulse the beam parameters usually have negligible time dependence so this is not an issue. 


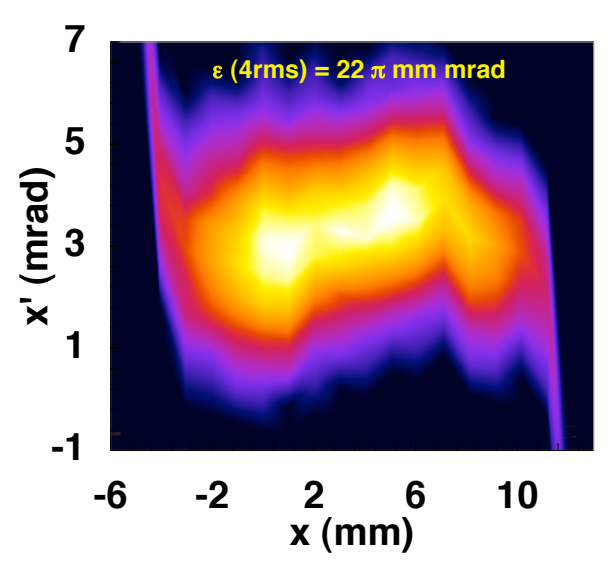

Figure 3.13. Sheared phase space distribution measured optically over a 500 ns gate in the middle of the $10 \mu$ s beam pulse.

\subsection{Longitudinal beam dynamics}

Longitudinal beam dynamics are inferred from measurements of the current profile, Marx voltage, and also directly measured using a spectrometer. The Marx voltage, used to extract the beam from the diode, is monitored through a capacitive divider which discussed later in Section 3.5.1.

A spectrometer, which is capable of measuring the longitudinal beam distribution, was first presented by R.E. Warren in 1947 [79]. The spectrometer consists of a cylindrical electrostatic dipole with a $90^{\circ}$ bend.

On the NDCX the beam passes through a $0.1 \mathrm{~mm} \times 5 \mathrm{~mm}$ slit plate at the entrance to the spectrometer. This slit reduces the transmitted beam current from $30 \mathrm{~mA}$, which is incident on the slit plate, to $\sim 10 \mu \mathrm{A}$ for current densities $\sim 20$ $\mathrm{A} / \mathrm{m}^{2}$. Although this is a reduction in signal $>3$ orders of magnitude, it is similar to our transverse phase space measurements. The beam ribbon traverses the $90^{\circ}$ bend of the electrostatic dipole and is detected at the focal plane (Fig. 3.14).

The dimensions of the spectrometer are shown in Fig. 3.14. A dipole radius $a$ of 


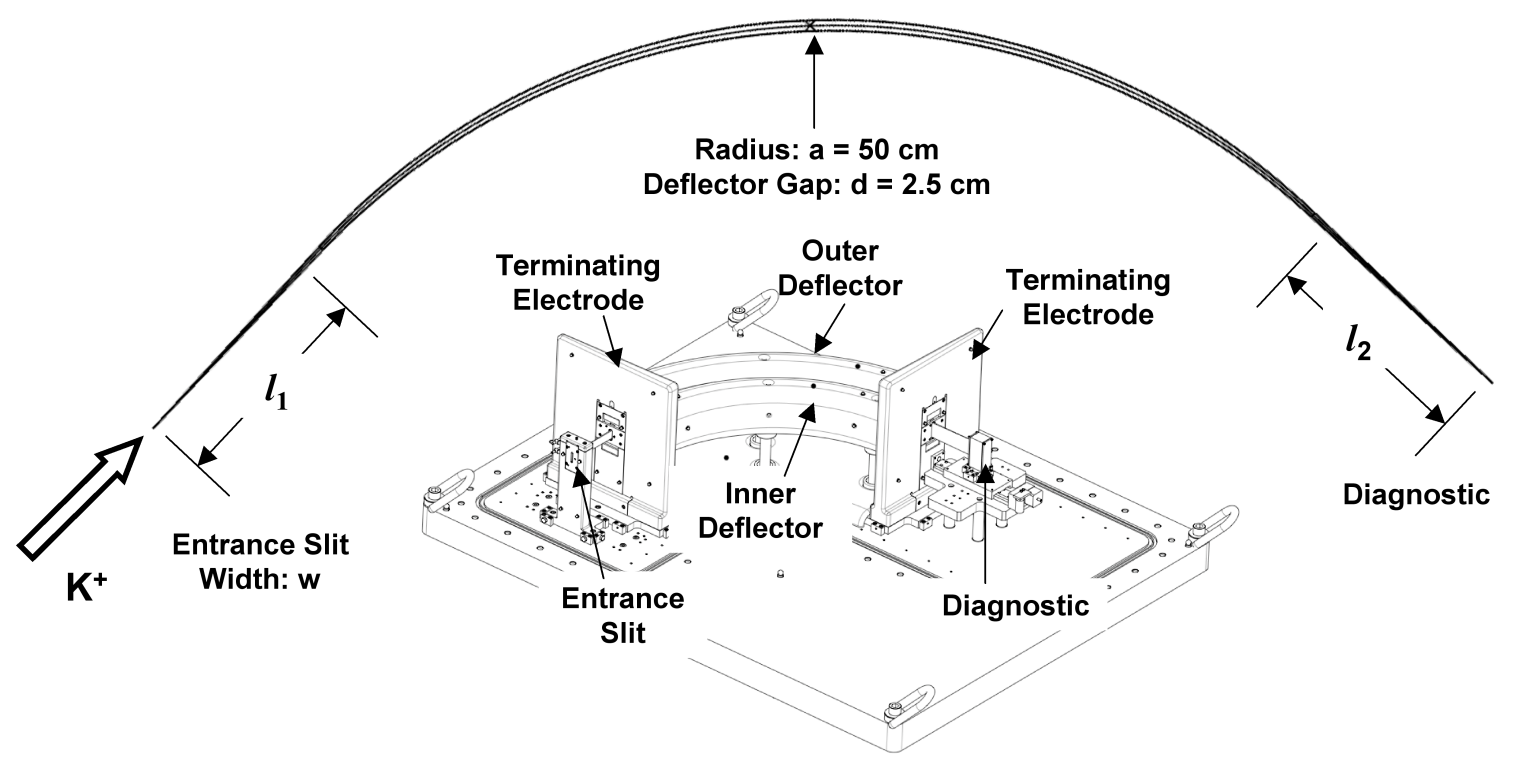

Figure 3.14. Schematic of the electrostatic energy analyzer used on NDCX.

$50 \mathrm{~cm}$ and the gap between the electrodes $d$ of $2.5 \mathrm{~cm}$ were chosen to measure up to a $1 \mathrm{MeV}$ beam. The corresponding energy on the dipole, $E$, for equal and opposite charge voltage on the plates, $V$, is

$$
E=\frac{V}{\ln \left(\frac{a+\frac{d}{2}}{a-\frac{d}{2}}\right)} .
$$

For this geometry $1 \mathrm{~V}=20 \mathrm{eV}$. The resolution $R$ of the spectrometer is defined by its geometry

$$
R=\frac{a(1+M)}{2 w M}
$$

where $w$ is the entrance slit width and $M$ is the magnification factor

$$
M=\frac{f}{l_{1}-g},
$$

where $f$ and $g$ are geometric factors

$$
f=\frac{a}{\sqrt{2}} \csc (\sqrt{2} \theta)
$$


Table 3.1. Parameters for the electrostatic energy analyzer used on the NDCX.

\begin{tabular}{|l|c|c|c|}
\hline Dipole Angle & $\boldsymbol{\theta}$ & ${ }^{\circ}$ & 90 \\
\hline Dipole radius & $\boldsymbol{a}$ & $\mathrm{cm}$ & 50 \\
\hline Dipole gap & $\boldsymbol{d}$ & $\mathrm{cm}$ & 2.5 \\
\hline Geometric factor & $\boldsymbol{g}$ & $\mathrm{cm}$ & -26.9 \\
\hline Geometric factor & $\boldsymbol{f}$ & $\mathrm{cm}$ & 44.4 \\
\hline Object Distance & $\boldsymbol{l}_{\boldsymbol{1}}$ & $\mathrm{cm}$ & 17.52 \\
\hline Image Distance & $\boldsymbol{l}_{\boldsymbol{2}}$ & $\mathrm{cm}$ & 17.52 \\
\hline Entrance slit width & $\boldsymbol{w}$ & $\mathrm{mm}$ & 0.1 \\
\hline Magnification & $\boldsymbol{M}$ & & 1 \\
\hline Resolution & $\boldsymbol{R}$ & $1 \mathrm{part}$ per & 4921 \\
\hline Energy Scale & $\boldsymbol{E}$ & $\mathrm{eV} / \mathrm{V}$ & 20 \\
\hline
\end{tabular}

$$
g=\frac{a}{\sqrt{2}} \cot (\sqrt{2} \theta)
$$

and $\theta$ is the dipole angle, which in this case is $90^{\circ}$. Geometric factors $f$ and $g$ are also related by the object distance $l_{1}$ and image distance $l_{2}$.

$$
f^{2}=\left(l_{1}-g\right)\left(l_{2}-g\right)
$$

$l_{1}$ and $l_{2}$ were chosen to be $17.52 \mathrm{~cm}$ based on the above equations and the chosen dipole radius and gap. The configuration used has the ability to resolve a beam distribution as narrow as $0.1 \mathrm{~mm}$ or $60 \mathrm{eV}$ for a $0.3-\mathrm{MeV}$ beam as shown in Table 3.1. A $0.05-\mathrm{mm}$ wide slit could also be used to double the resolution.

This is the theoretical resolution; the actual resolution and absolute energy calibration of the diagnostic is dependent on several constraints. First, the mechanical tolerances and alignment of internal components must be aligned within $0.1 \mathrm{~mm}$ or better if possible. The high voltage on the dipole electrodes must be calibrated within $\Delta \mathrm{V} / \mathrm{V}=2 \times 10^{-4}$. Space charge effects must be eliminated. Finally, beam and plasma loading which can cause voltage drop on the electrodes must be eliminated as well. 
All of these specifications were met for our measurements, however there are additional constraints for absolute energy measurements. First, there are finite fringe fields at the beginning and end of the dipole bend (near the terminating electrodes). These fringe fields cause an effective increase or decrease in the dipole angle and this must be quantified. If the dipole bend is not exactly $90^{\circ}$ the beam will show up to the left or right of the expected transverse location on the focal plane. An additional check of the effective steering energy of the dipoles can be calibrated with a known radiative source of energy.

\subsubsection{Longitudinal temperature}

Transverse beam distributions [Fig. 3.15(a)] transmitted to the diagnostic plane provide a measurement of longitudinal temperature of the beam. These measurements were made optically with a $100-\mu \mathrm{m}$ alumina scintillator similar to that described in Sections 3.1.3 and 3.2.1. The beam-induced light emission was captured by an image intensified gated-CCD camera. The width $\Delta x$ of the transverse distribution measured at the diagnostic plane is a function of the geometry of the spectrometer and the energy spread $\Delta E$ of the beam.

$$
\Delta x=\frac{a \Delta E}{2 E_{o}}(1+M)
$$

where $E_{o}$ is the particle energy that is transported through the center of the spectrometer. $\mathrm{A} \Delta \mathrm{E}=1 \mathrm{keV}$ for a $300 \mathrm{keV}$ beam is equivalent to a $1.67 \mathrm{~mm} \Delta \mathrm{x}$ at the focal plane. This relation is also used to determine the energy of the beam for optical measurements. The energy spread is shown in Fig. 3.15(b). 

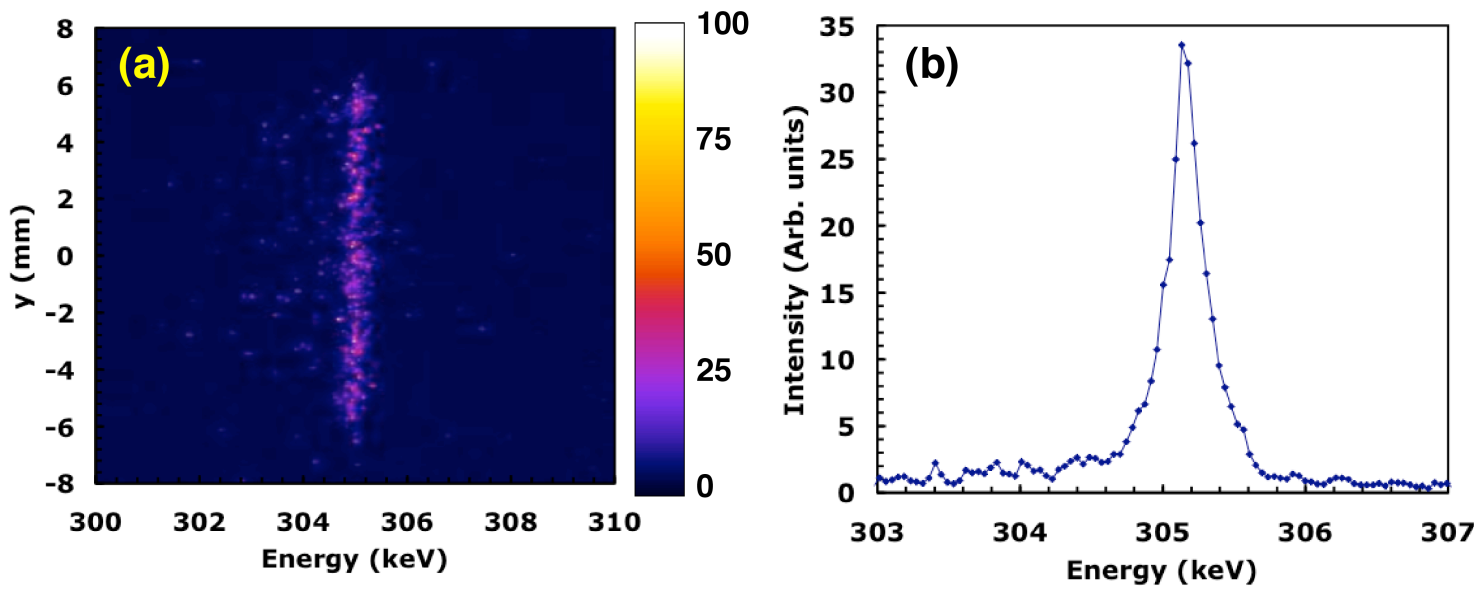

Figure 3.15. (a) Transverse slice of a $305-\mathrm{keV}$ beam integrated over a 500 ns gate in the middle of a $3 \mu$ s pulse; (b) intensity of the slice projected onto the energy axis.

\subsubsection{Longitudinal phase space}

These transverse beam distributions are also measured as a function of time with an image intensified streak camera. In principle the streak camera has the ability to resolve the longitudinal phase space from the transverse beam distributions for a single shot. We used a Hamamatsu streak C7700 [80] which has a temporal resolution of 5 ps and a $1344 \times 1024$ CCD pixel array. The spatial resolution of the camera is flexible and depends on the optical setup used.

We have taken measurements with the slit of the streak camera set to integrate the beam over 250 ns slices throughout the $3 \mu$ s beam pulse. This cuts down the amount of light collected from previous measurements with the image intensified gated-CCD camera by a factor of four. This is because we are integrating over half as long of a time window and the slit is only capturing $4 \mathrm{~mm}$ of the $1 \mathrm{~cm}$ high beam distribution. An average longitudinal phase space distribution from 10 streaked images is shown in Fig. 3.16(a). The beam energy fluctuations from shot to shot can induce a false widening to the width of the intrinsic distribution. The light collection efficiency was 

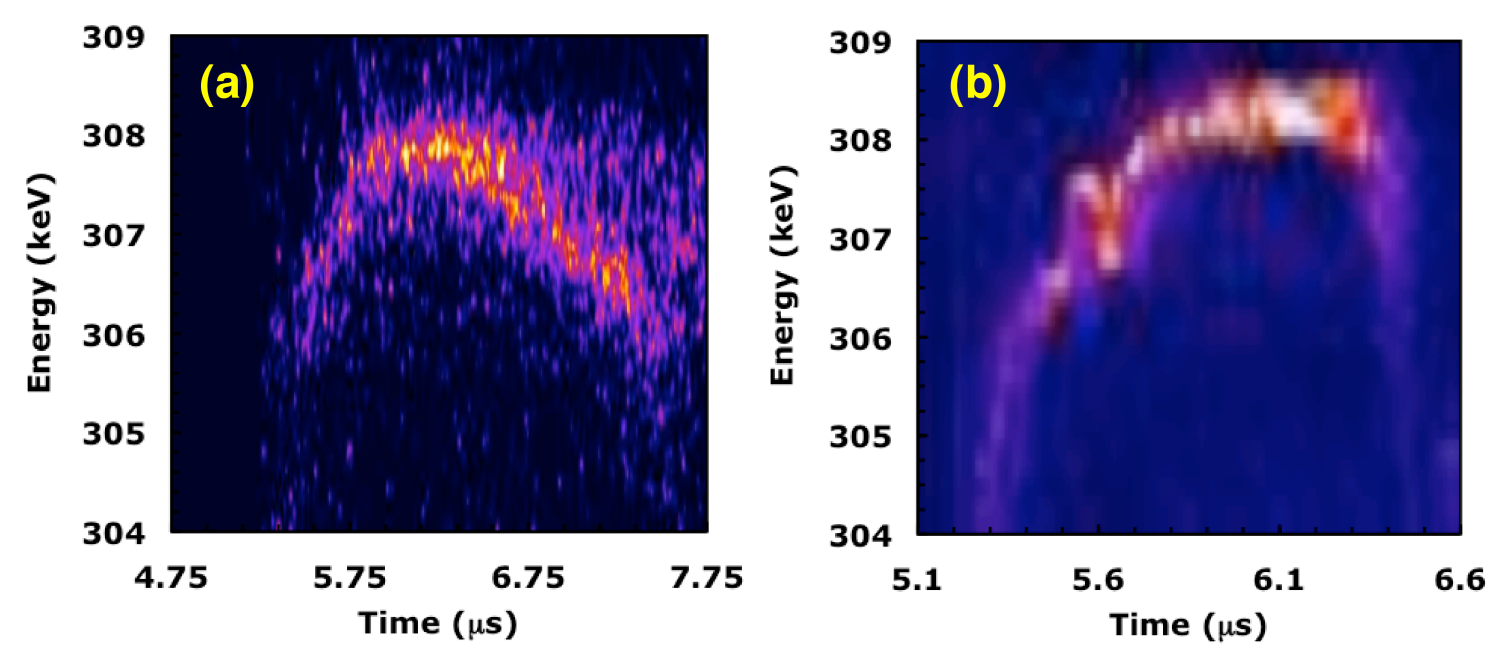

Figure 3.16. Longitudinal phase space distribution of the NDCX beam (a) with a 3 $\mu$ s pulse streaked in time and; (b) a $2 \mu$ s pulse measured with two slits.

low for these measurements and should be improved in the future to maximize the use of the CCD. The CCD for this streak camera is $>$ four times larger than the image intensified gated-CCD camera so a closer coupling via additional focusing lenses or a fiber optic bundle is necessary.

Another method of measuring the longitudinal phase space is made using a slit and a Faraday collector (slit-cup) at the focal plane of the spectrometer. This method is similar to that used for transverse phase space measurements. The upstream slit is the same as described above $(0.1 \mathrm{~mm} \times 5 \mathrm{~mm})$ and the downstream slit was increased to $0.1 \mathrm{~mm} \times 10 \mathrm{~mm}$ and the downstream slit was increased to $0.1 \mathrm{~mm} \times 10 \mathrm{~mm}$ to compensate for beam expansion.

Step sizes for these measurements could be as small as the resolution of the spectrometer $(60 \mathrm{eV})$ but larger steps $(200 \mathrm{eV})$ were taken due minimum energy variation of the beam from shot to shot. The slit-cup generally measured tens of millivolts ( $\mu \mathrm{A}$ of current) and the $\mathrm{S} / \mathrm{N}$ was close to 10 when configured to measure secondary 
electrons leaving the collector and using an amplifier. The measured distribution for a $2 \mu$ s beam pulse using this technique is shown in Fig. 3.16(b).

These two separate measurements of the longitudinal phase space are compared to one another in Section 7.4. All of the longitudinal beam dynamics measurements with the spectrometer are correlated with the Marx voltage waveform which is used to extract the beam in Section 7.6.

\subsection{Electron cloud and gas measurements}

\subsubsection{In-bore cylindrical electrodes}

Extensive studies of electron cloud and gas effects in the four-solenoid lattice on NDCX were done with the apertured 26-mA beam using new cylindrical electrodes inside the beam pipe (electron cloud diagnostics; Fig. 3.17). These electron cloud diagnostics consisted of four short (8.45-cm long) cylindrical electrodes in the center of each solenoid magnet (solenoid electrodes 1,3,5, and 7) and the three longer (25.4-cm long) cylindrical electrodes in the gaps between magnets (gap electrodes 2,

4 , and 6$)$. These electrodes are short compared to the beam bunch length $(\sim 1.2$ $\left.\times 10^{3} \mathrm{~cm}\right)$ and have an inner radius $(3.6 \mathrm{~cm})$ slightly smaller than the beam pipe radius of $4.3 \mathrm{~cm}$. Strategically placed gap electrodes intercept the maximum amount of expanding magnetic flux between magnets A 13-cm long cylindrical electrode was placed in the exit of solenoid 4 (electrode 8; Fig. 3.17) to intercept the expanding magnetic flux at the exit of solenoid 4 . Thus, electrode 8 has a similar function to a gap electrode. A pair of parallel plates was used just upstream of the intercepting diagnostics to suppress electrons and measure the dynamics of beam-induced gas desorption, ionization, and electron emission. 


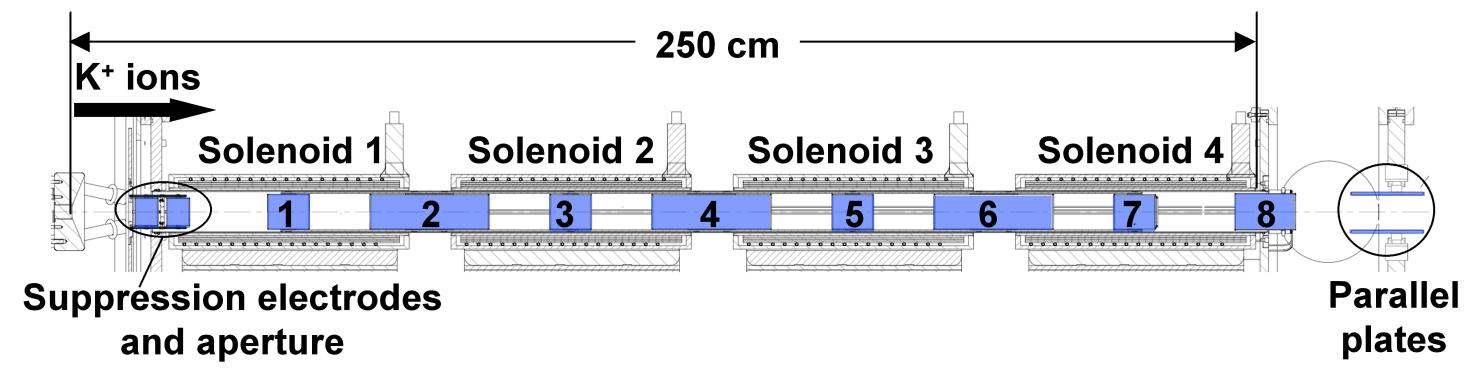

Figure 3.17. Layout of the aperture and suppression electrodes, electron cloud diagnostics: solenoid electrodes (1, 3, 5, and 7), gap electrodes (2, 4, 6, and 8); and parallel plate diagnostic relative to the four-solenoid lattice. All the diagnostics have cylindrical symmetry except for the parallel plate diagnostic.

These cylindrical electrodes measured a positive capacitive image current of the beam as it entered the diagnostic and negative capacitive image current of the beam as it exited (Fig. 3.18). The electrodes collected charge throughout the pulse based on the bias configuration and location of the diagnostic along the focusing lattice. These cylindrical electrodes were independently biased between $\pm 1 \mathrm{kV}$. The solenoid electrodes $(1,3,5$, and 7$)$ were biased negatively to repel electrons, while the gap electrodes $(2,4,6$, and 8$)$ were biased positively to clear electrons from intercepted field lines and suppress emission. Reversing the biases trapped electrons that were emitted from the gap electrodes between magnets. The details of the measurements made and the effects of these diagnostics on the beam dynamics are explained in Section 5.4.

\subsubsection{Electrostatic dipoles}

A pair of polished stainless steel parallel plates $15 \times 15 \mathrm{~cm}^{2}$ spaced $7.5 \mathrm{~cm}$ apart (Fig. 3.19) and an intercepting diagnostic were used to make measurements of beam induced gas desorption, ionization, and electron emission with the apertured 26-mA beam. 


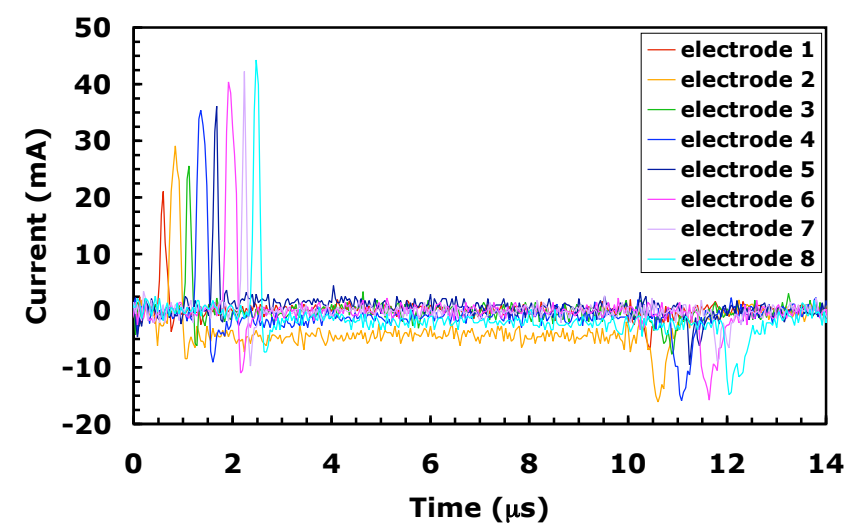

Figure 3.18. Electron cloud diagnostic signals as a function of time.

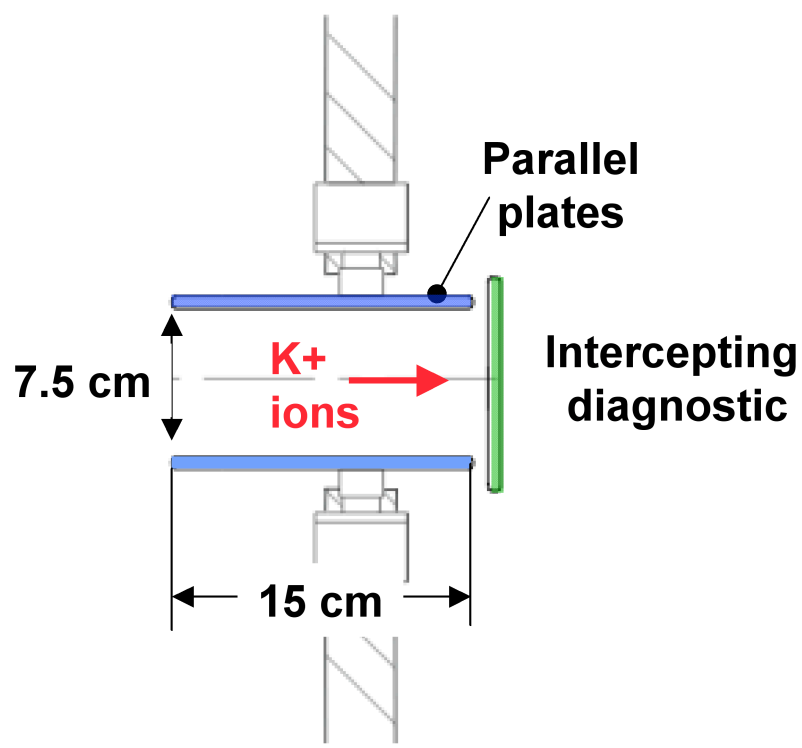

Figure 3.19. Sketch of the experimental setup using the parallel plates and intercepting diagnostic to measure beam induced gas desorption, ionization, and electron emission. 
Two separate diagnostics, a stainless steel plate and a copper plate, were used in consecutive data sets to intercept the beam. Using two separate materials tested whether the amount of gas desorbed and electrons emitted was strongly dependent on material composition. Each plate was at least 0.5 -mm thick and 4-cm wide to fully intercept the $1.5-\mathrm{cm}$ wide beam. The plates were prepared in the same manner; first they were chemically cleaned by degreasing at $50{ }^{\circ} \mathrm{C}$ with ultrasonic agitation, followed by rinsing in cold demineralized water, rinsing with alcohol, and finally, drying at room temperature. This method is typical for any of our vacuum components. The intercepting plates (targets) and parallel plates were capacitively monitored for all measurements and the details of the measurements made are discussed in Section 5.5.

\subsection{Pulsed power diagnostics}

Pulsed power provides the energy for all of the hardware used to manipulate the beam on the NDCX. The beam is accelerated and extracted through the diode using a pulsed Marx capacitor bank. The beam is focused and transported downstream of the diode by pulsed solenoid magnets. Pulsed magnetic dipoles correct the beam centroid motion caused by misaligned focusing elements. A pulsed induction cell provides the velocity tilt applied to the beam for axial compression. Finally, the plasma sources, which provide the current neutralizing plasma that enables us to focus to emittance

limited spots are also pulsed. Each one of these pulsed power sources is individually monitored during operation to verify proper operation. 

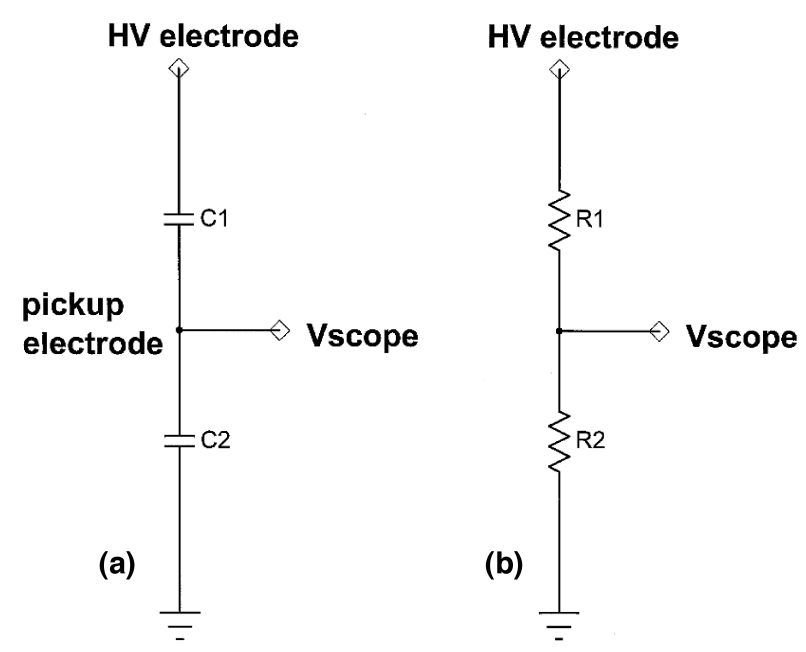

Figure 3.20. Schematic of (a) a capacitive divider and; (b) a resistive divider used on NDCX.

\subsubsection{High voltage monitors}

The high-voltage pulses from the Marx and the induction cell are monitored through a capacitive divider shown in Fig. 3.20(a). The capacitance between the pickup electrode and the voltage output is $C 1$. The capacitance from the cable and the pickup electrode to ground is $C 2$. $C 2$ is chosen to be $>>C 1$ to cause a large voltage drop from the voltage output to the scope as shown below

$$
V_{\text {scope }}=\frac{V \cdot C 1}{C 1+C 2}
$$

where $V_{\text {scope }}$ is the voltage to the scope and $V$ is the high voltage source. Each of these diagnostics is terminated in high impedance to avoid loading down the signal. The voltage drop on the Marx capacitive divider was calibrated at $28.3 \mathrm{kV} / \mathrm{V}$ and for the induction cell it was $10.9 \mathrm{kV} / \mathrm{V}$. Each were calibrated using a high voltage probe.

The high-voltage pulses from the pulse forming network used to drive the ferroelectric plasma source is monitored through a resistive divider shown in Fig. 3.20(b). 
Table 3.2. Details of different current pulsers on NDCX and the specification of the current transformers used to monitor the current signals.

\begin{tabular}{|c|c|c|c|c|c|c|c|c|c|}
\hline Pulser & $\begin{array}{c}\text { Pulse } \\
\text { Length (ms) }\end{array}$ & $\begin{array}{l}\text { Rise } \\
\text { Time }\end{array}$ & $\begin{array}{c}\text { Peak } \\
\text { Current } \\
\text { (kA) }\end{array}$ & Brand & Model & $\begin{array}{l}\text { Output } \\
\text { (V/A) }\end{array}$ & $\underset{\text { Current }}{\operatorname{Max}}$ & $\begin{array}{c}\text { Max } \\
\text { Charge } \\
\text { (C) }\end{array}$ & $\begin{array}{c}\text { Minimum Rise } \\
\text { Time (ns) }\end{array}$ \\
\hline Transport Solenoids & 4 & $2 \mathrm{~ms}$ & 13 & Pearson & $301 X$ & 0.01 & 50 & 22 & 200 \\
\hline Dipole Magnets & 2 & $600 \mu \mathrm{S}$ & 0.2 & Pearson & 411 & 0.1 & 5 & 0.2 & 20 \\
\hline FCAPS & 0.4 & $20 \mu \mathrm{s}$ & 0.5 & Pearson & 110 & 0.1 & 5 & 0.5 & 20 \\
\hline FEPS & $3 \times 10^{-3}$ & $500 \mathrm{~ns}$ & 0.5 & Ion Physics Corp & CM-1-M & 0.01 & 50 & 0.05 & 60 \\
\hline
\end{tabular}

The resistance from the voltage output is $R 1$. The resistance from the pickup to ground is $R 2$. $R 1$ is selected to be $>>R 2$ to cause a large voltage drop from the voltage output to the scope as shown below

$$
V_{\text {scope }}=\frac{V \cdot R 2}{R 1+R 2}
$$

The measured voltage drop for the resistive divider used to monitor the ferro-electric plasma source is $3.0 \mathrm{kV} / \mathrm{V}$.

\subsubsection{Current monitors}

Current monitors are used to detect the drive currents of the transport solenoids, dipole magnets, filtered-cathodic plasma sources (FCAPS), and ferro-electric plasma sources (FEPS). The current monitors used on the NDCX were typically Pearson current transformers [81]. Each current monitor has different specifications depending on the amplitude, pulse width and rise time of the pulse. Similar to any other transformer for the ideal case the power is conserved. Since we are monitoring current through a single cable (primary) used to drive the system, the voltage of the secondary is stepped up by the number of turns in the secondary. As a result the current is stepped down by that amount. The details of the different pulsers and current transformers we use on the NDCX are listed in Table 3.2. 


\subsection{Data acquisition system}

The diagnostics listed earlier in Chapter 3 take measurements of the beam current, energy, longitudinal and transverse beam dynamics, electron cloud and gas effects and pulsed power used to drive the system. These diagnostics were monitored by a series of Tektronix oscilloscopes [82]. The scopes were all triggered simultaneously by firing the Marx capacitor bank, which was the beginning of extraction of the beam from the diode. The scopes were configured to maximize time resolution and voltage resolution for each diagnostic. A series of different LabVIEW [83] programs were written to instantaneously sample the data acquired by our Tektronix scopes. Therefore, each time the beam was pulsed $(\geq 0.05 \mathrm{~Hz})$ data was sampled by the scopes and saved by a LabVIEW program.

Two additional LabVIEW programs were written to measure the transverse beam dynamics. The first was an older program written to use the upstream slit and downstream slit-cup to measure the beam profile, transverse phase space, and emittance (Section 3.2) [84]. The program had drivers which moved mechanical step motors automatically; these motors controlled the motion of the slits to $<0.1 \mathrm{~mm}$. This program also had the ability to save waveforms. So once a profile or phase space boundary was selected, the program was able to automatically trigger the system, acquire data, and map out the transverse beam dynamics.

After considering the amount of time consumed by the previous method and the technology at hand, it was advantageous to upgrade the program to control the Princeton Instruments CCD camera [78]. The program still operated the step motor for the upstream slit, but the downstream slit-cup was replaced by an alumina scintillator. This removed the need to map out all of the angles downstream with a slit-cup. With the LabVIEW drivers provided by Princeton Instruments [85], the 
program was upgraded to automatically trigger the system, acquire both optical and beam signal data, and map out the transverse beam dynamics.

Optical images were also acquired on a single shot basis with WinView [85], an imaging software provided by Princeton Instruments. This method of acquisition was used if operating parameters were being changed frequently and only a limited amount of data was acquired.

\subsection{Presentation of the data}

The data acquired on the NDCX required some processing. This meant background subtractions were performed, scaling the signals based on calibrated values, and looking for trends in the data.

\subsubsection{Oscilloscope signals}

All of the diagnostic signals monitored on the NDCX beam line measure voltage drop. This voltage drop either corresponds to voltage on a high-voltge monitor, current monitored through a transformer, or current collected by a diagnostic or light output. Each of these signals is monitored by a scope, which can generate a small voltage bias offset (usually a couple of $\mathrm{mV}$ ) of the diagnostic signal. Performing a background subtraction of this bias offset is especially critical for diagnostics with low S/N (i.e. the pinhole Faraday cup and phototube).

When performing the background subtraction, the raw data is compiled into a spreadsheet. Each individual shot has a separate background subtraction that must be performed because the bias offset of the waveform recorded from the scope, which may be positive or negative, varies from shot to shot. 
This background subtraction can be done in two ways:

1. Subtract the average of all data points preceding the rising part of the waveform from the whole waveform. This resets the bias offset (preceding signal) to zero and is valid for the rest of the waveform providing that a constant background is a good representation.

2. Subtract the average of all data points preceding and following the signal from the whole waveform to reset the bias offset (preceding and trailing signal) to zero.

Method 2 is used only if there is insufficient data recorded before the waveform to obtain an accurate average background level. Also if method 2 is used, one must be careful that the average value is not influenced by any undershoot, or by any slow time constants (e.g., charge dissipation from gas and electron effects in the detector) that would invalidate this approach.

\subsubsection{Optical images}

Similar to signals acquired by the oscilloscopes, optical images also have background light that must be subtracted. The optical data acquired from both the CCD camera and streak camera were analyzed using an image processing and analysis program in java called Image $\mathrm{J}[86]$. This software has the ability to analyze individual images and also groups of images. Several java programs and macros have been written for the statistical analysis of the data. A specific algorithm was written to analyze current density $[\mathrm{J}(\mathrm{x}, \mathrm{y})]$ distributions and calculate the 2rms radius, a, and the centroid offset, $\langle\mathrm{x}\rangle$, for a given background subtraction.

Trends in the data must be understood when analyzing current density $[\mathrm{J}(\mathrm{x}, \mathrm{y})]$ 

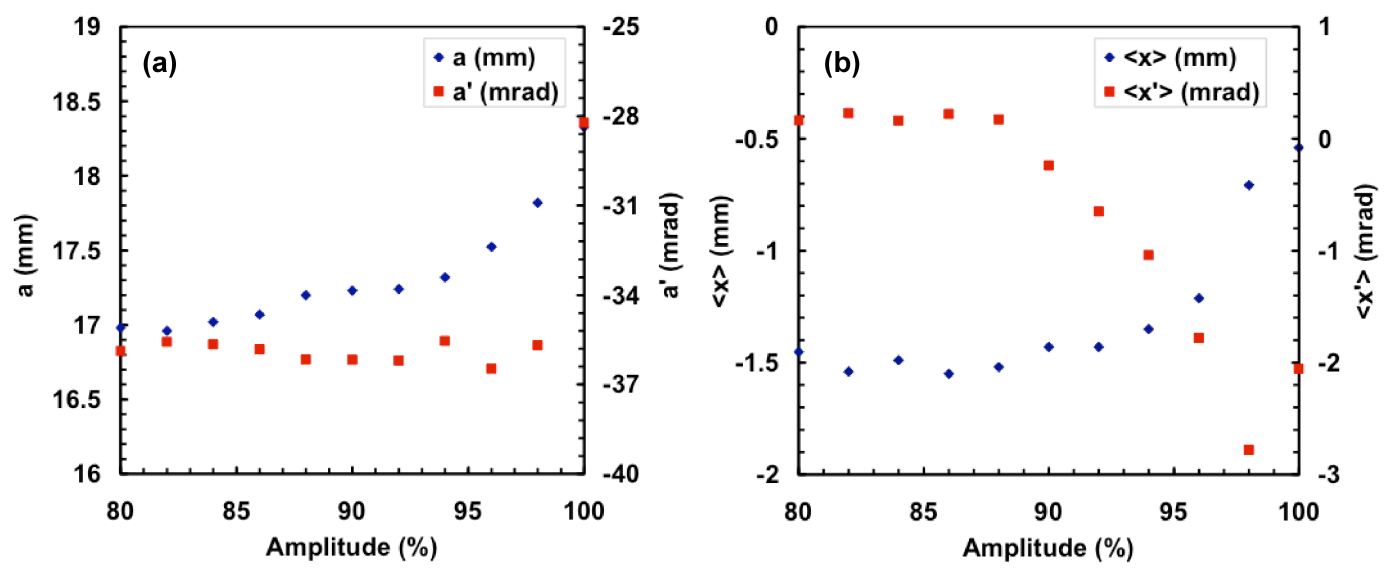

Figure 3.21. Thresholding of the (a) envelope parameters and; (b) centroid offsets for optical phase space measurements analyzed with Image J.

distributions. Generally, $100 \%$ of the signal amplitude cannot be used to calculate a and $\langle\mathrm{x}\rangle$. A threshold of the background data must be determined and beyond this will be the most accurate representation of the true beam parameters. Examples of some of the trends that are looked for are shown in Fig. 3.21. The experimenter must find the threshold or knee in the data below $100 \%$ of the signal amplitude, in these examples that is around $90 \%$. At this point the beam parameters are relatively constant.

\subsubsection{Transverse beam dynamics}

As described in Section 3.2, there are two different diagnostic techniques used to measure the transverse beam dynamics, the optical method and the slit-cup method. There is a graphical user interface for analyzing the data obtained from each of these methods.

Data acquired with the slit-cup is analyzed with a script that was written in MATLAB [87]. Two separate scripts were written, one was written to analyze the 


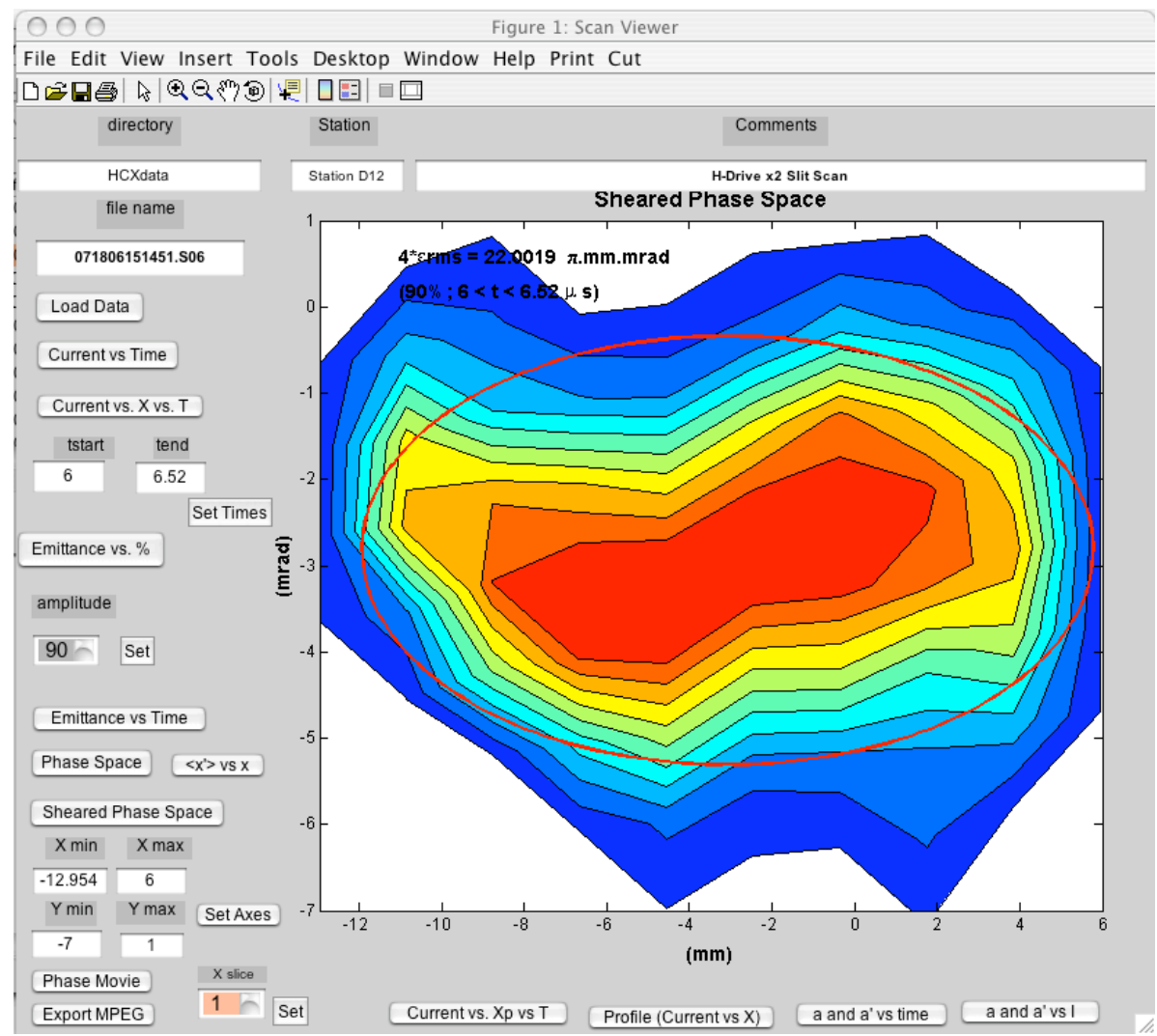

Figure 3.22. Screen capture of the MATLAB user interface to analyze the transverse phase space. 
transverse profiles and another was written to analyze the transverse phase space. Each have the ability to calculate the 2rms radius, a, and the centroid offset $\langle\mathrm{x}\rangle$, however only the script that analyzes the transverse phase space can calculate the 4rms emittance, $\epsilon_{4 \mathrm{rms}}$, envelope angle, $\mathrm{a}^{\prime}$, and the centroid offset $\left\langle\mathrm{x}^{\prime}\right\rangle$. The graphical user interface used to analyze the data acquired by the slit-cup is shown in Fig. 3.22. The data analyzed in the MATLAB scripts can be exported to ASCII files and image files can be saved in nearly any format (.pdf, .eps, .jpg, .tiff.).

Two different algorithms were written in java for the analysis of optical data from transverse phase space measurements. One was capable of reconstructing the 4-D transverse phase space and providing a particle distribution. Each have the ability to calculate the $4 \mathrm{rms}$ emittance, $\epsilon_{4 \mathrm{rms}}$, envelope parameters, a, a', and centroid offsets $\langle\mathrm{x}\rangle,\left\langle\mathrm{x}^{\prime}\right\rangle$. The graphical user interface used to analyze the optical data is shown in Fig. 3.23. The data analyzed in Image $\mathrm{J}$ can be exported to ASCII files and image files can also be saved in nearly any format.

Similar to the optical in Section 3.7.2, trends in the beam dynamics data must be understood. All of the data has a base or background level that must be subtracted. In the optical data, again, there can be background light that must be subtracted, and in the data acquired with the slit-cup there can be electrical noise. Generally, $100 \%$ of the signal amplitude cannot be used to calculate the $4 \mathrm{rms}$ emittance, $\epsilon_{4 \mathrm{rms}}$, envelope parameters, a, $\mathrm{a}^{\prime}$ and centroid offsets $\langle\mathrm{x}\rangle,\left\langle\mathrm{x}^{\prime}\right\rangle$. 


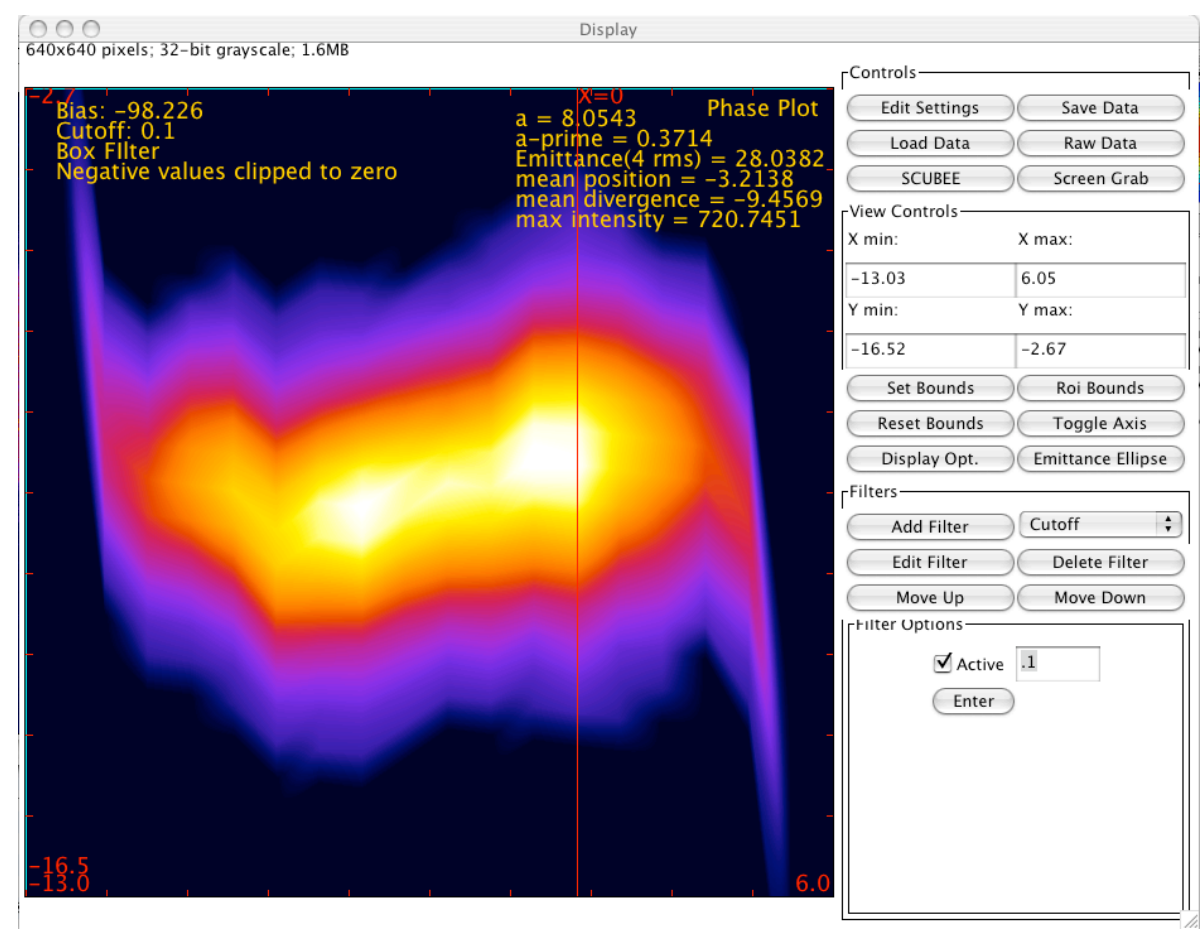

Figure 3.23. Screen capture of the Image $J$ user interface to analyze the transverse phase space. 


\section{Chapter 4}

\section{Numerical simulation tools}

A good portion of the success achieved on the NDCX is attributed to the insight provided by numerical modeling. Without these tools it is difficult, if not impossible, to design and setup the experiment. Described below are two numerical techniques used to model, predict, and understand the beam dynamics. The first method described solves the differential equations, which model the longitudinal and transverse beam envelopes. The second method uses particle-in-cell (PIC) techniques to model the beam dynamics.

\subsection{Envelope codes}

All of the experiment, except for the acceleration through the diode, was modeled by solving the envelope equations (Eqs. 2.37, 2.67), which describe the fluctuation of the radial envelope and bunch width of a charged particle beam with finite emittance, space charge, and applied focusing fields. 


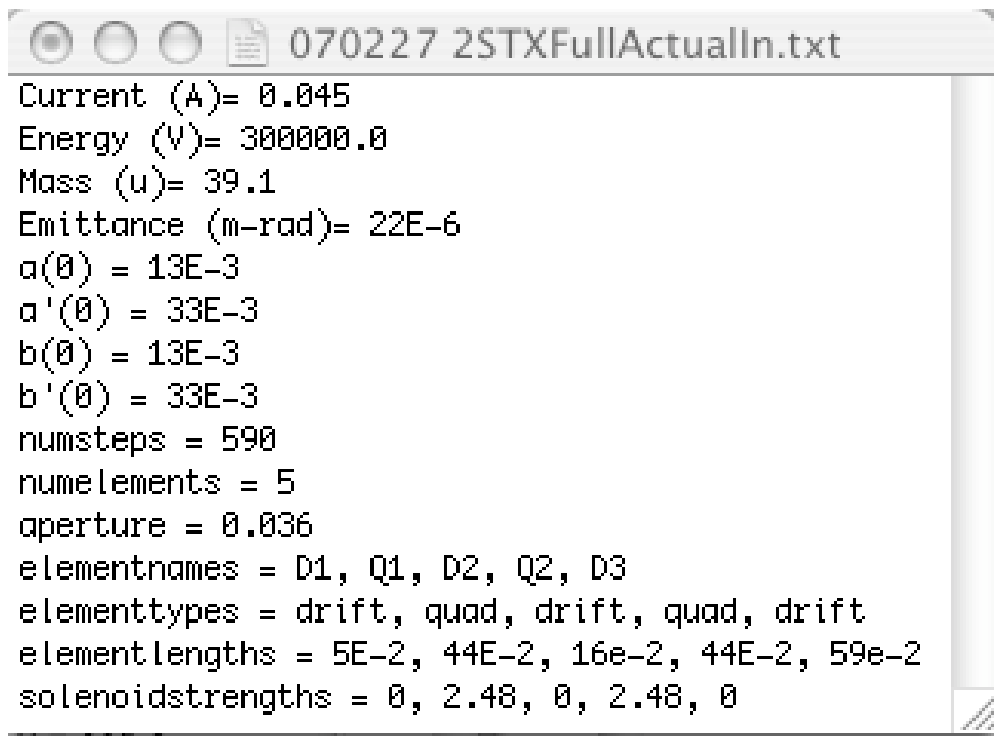

Figure 4.1. Example of an input text file with NDCX beam parameters and initial conditions. Units are in meters, radians, and Tesla.

\subsubsection{Java based}

A java based code was developed to solve the transverse envelope equation (Eq. 2.37) using the 4th order Runge-Kutta method [88, 89]. Runge-Kutta is an iterative method used to give an approximate solution to ordinary differential equations.

This java based code has a user-friendly interface which can be compiled on any Macintosh, Linux, or Windows operating system. The code loads a simple input deck, which is a text file, that contains the beam parameters and initial conditions (Fig. 4.1). The input deck also contains a description of the lattice parameters, which consist of drift lengths and focusing element lengths and strengths. Once the input deck is loaded the envelope is calculated. If one wishes to change the beam parameters, focusing element strengths, or initial conditions of the beam, they can do so by making a selection from the user interface (Fig. 4.2) or writing a new input deck. 


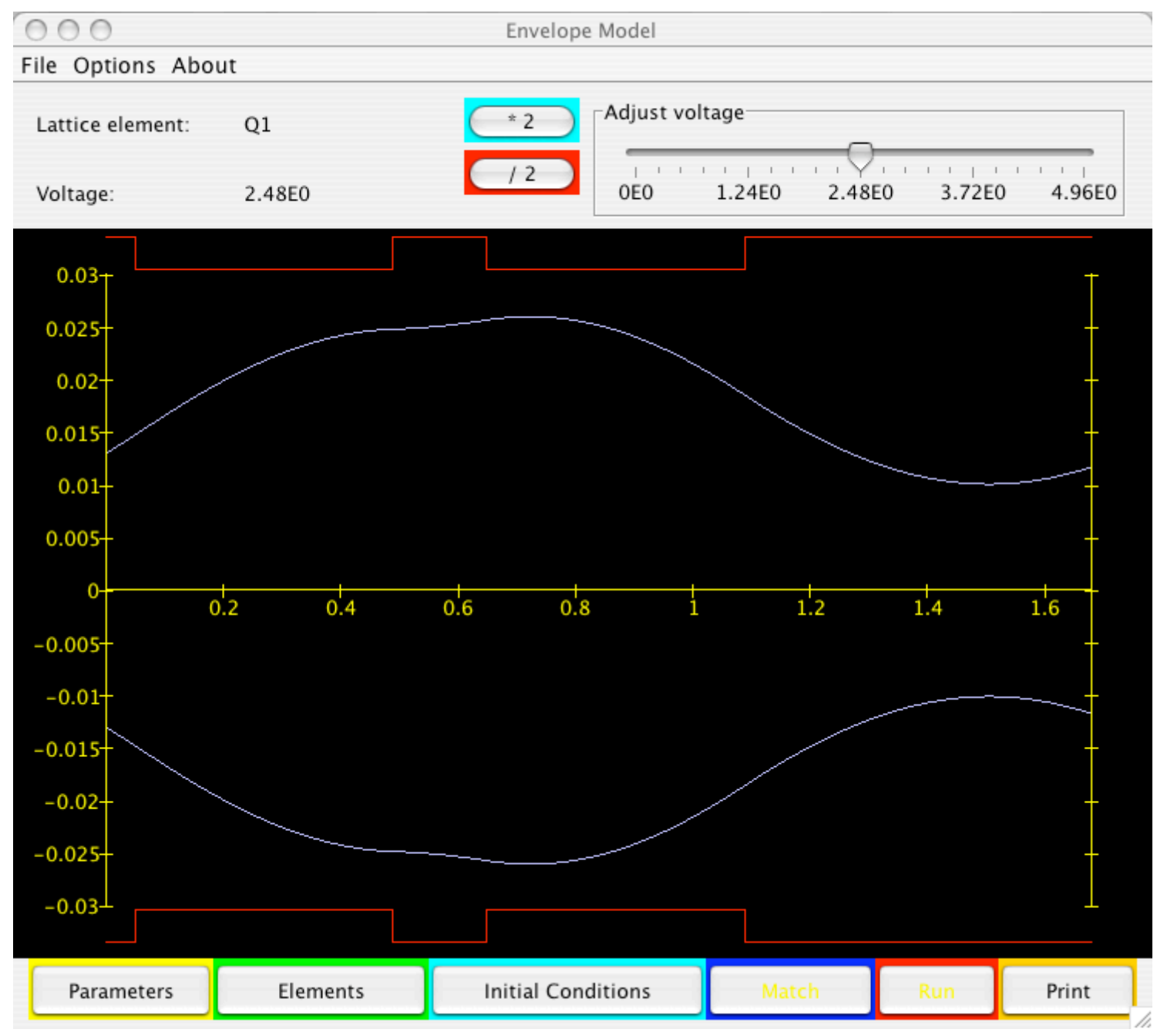

Figure 4.2. Screen capture of the envelope java code user interface. 
The code was modified from its original version of modeling electrostatic quadrupole focusing elements to model solenoids on the NDCX. A square field approximation is made for the solenoids. This is done for simplicity, although not entirely accurate, the effective length of the hard edge solenoid, $l$, from Eq. (2.57) is equal to $l$ of the actual solenoid with fringe fields

$$
l=\frac{\int B \cdot d z}{B_{\max }}
$$

where $B$ is the magnetic flux density along the solenoid and $B_{\max }$ is the maximum magnetic flux density. Thus, the overall focusing effect of the solenoid is correct, but the calculated beam envelope in the fringe field region of the solenoids is generally inaccurate by $>1 \%$. However, outside of the fringe, where most of the beam envelope parameters are measured, the calculated values match up to the experiment very well as shown in Chapter 5 .

One negative characteristic of this code is that it only models the transverse beam dynamics for a single beam current per input deck. This is problematic for the NDCX because there is axial bunching and neutralization of the beam current downstream of the focusing lattice. If one wishes to model the transverse beam dynamics of a neutralized beam, a separate input deck must be written with the appropriate initial conditions and the current must be set to nearly zero so the emittance dominates. If one wishes to model single particle motion, the current and the emittance must be set to zero. Note at this point the transverse envelope equation is now simplified to the paraxial ray equation.

\subsubsection{MathCAD based}

A MathCAD [90] worksheet was written to solve both the transverse and longitudinal envelope equations (Eqs. 2.37, 2.67) in order to model simultaneous transverse 
and longitudinal focusing. The worksheet itself contains the beam parameters, initial conditions, and lattice parameters. The lattice parameters consist of drift lengths, focusing element lengths and strengths, a velocity tilt, and neutralization section. Each of these parameters is easy to change in the worksheet individually, but if one wishes to compare cases with multiple parameter changes a separate worksheets must be written. This worksheet also uses the 4th order Runge-Kutta method [89] to solve the envelope equations.

We know from Section 2.5 that the velocity tilt, which axially compresses the beam, also defocuses the beam transversely and causes a chromatic aberration. The analytic solution for the time dependent effects on the radial velocity, axial velocity, and beam envelope angle are all included in the worksheet. Assuming the number of particles in 4-D transverse phase space are conserved separately from 2-D longitudinal phase space, the worksheet solves the 6-D envelope for a single case, which corresponds to a single energy slice.

Fig. 4.3 shows an example of a 6-D envelope calculation in the worksheet for a single energy slice. The radius and bunch length are solved after they are imparted with the velocity tilt at $\mathrm{z}=284 \mathrm{~cm}$. For this case, the beam is dominated by space charge for $26 \mathrm{~cm}$ until the current is neutralized by a background plasma at $\mathrm{z}=310 \mathrm{~cm}$. Next, the neutralized beam drifts $95 \mathrm{~cm}$ before entering a 10-cm long final focusing solenoid at at $\mathrm{z}=405 \mathrm{~cm}$, which focuses the beam on a target $13 \mathrm{~cm}$ downstream of the solenoid at at $\mathrm{z}=428 \mathrm{~cm}$.

A more complicated worksheet could be developed to analyze the time dependent axial velocities and beam envelope angles, but it was determined that using a MATLAB [87] script was more efficient. 

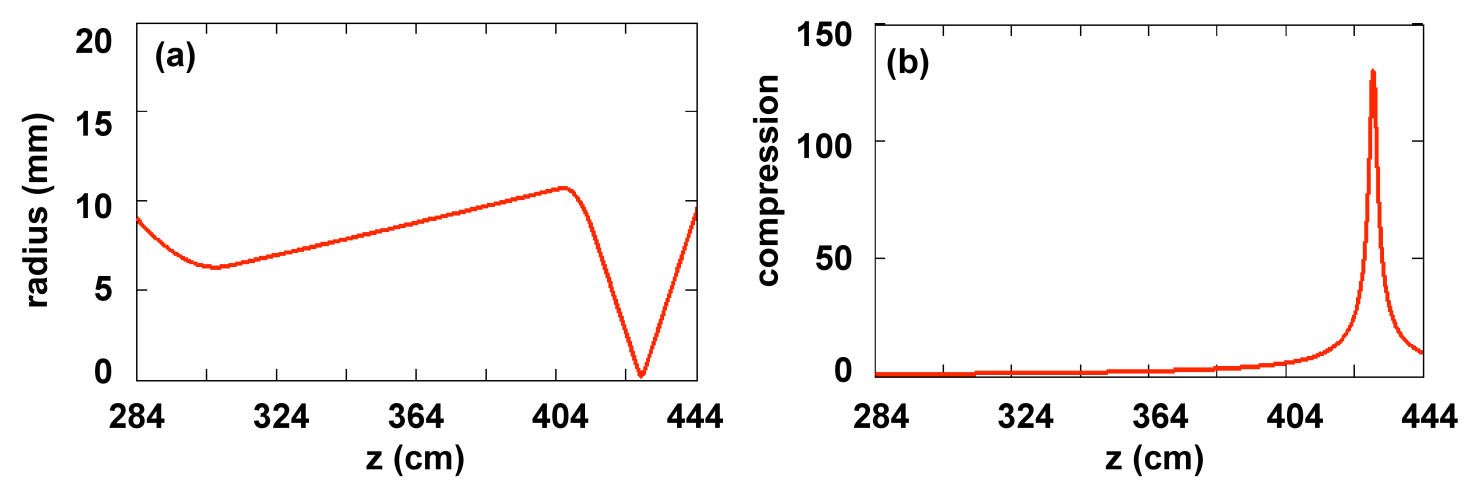

Figure 4.3. Calculated (a) transverse envelope and; (b) axial compression ratio downstream of the induction gap.

\subsubsection{MATLAB based}

In order to model the chromatic aberrations induced by the velocity tilt a MATLAB script was written to solve the transverse envelope equation for different energy slices. A matrix of time dependent axial velocities and beam envelope angles was constructed and solved using this method. These individual energy slices are modeled as equal slices of charge from the initial bunch that is compressed. These energy slices are summed up as individual transverse Gaussian slices at the focus. All of the slices have a different envelope, but the energy slice with no $\Delta \mathrm{v}_{\mathrm{z}}(\mathrm{E}=300 \mathrm{keV})$ is designed to focus on target (Fig. 4.4). As a result, the lower energy bunches come to a focus upstream (are diverging at the target) and the higher energy bunches come to a focus downstream (are converging at the target) [Figs. 4.4(b) \& (c)]. The composite bunch is a peaked distribution composed of short, fat Gaussian distributions from beam energies with large $\Delta \mathrm{v}_{\mathrm{z}}$ and tall, narrow Gaussian distributions from beam energies with small $\Delta \mathrm{v}_{\mathrm{z}}$ (Fig. 4.5).

Details of these calculations are described in Section 6.4. The expected increase 

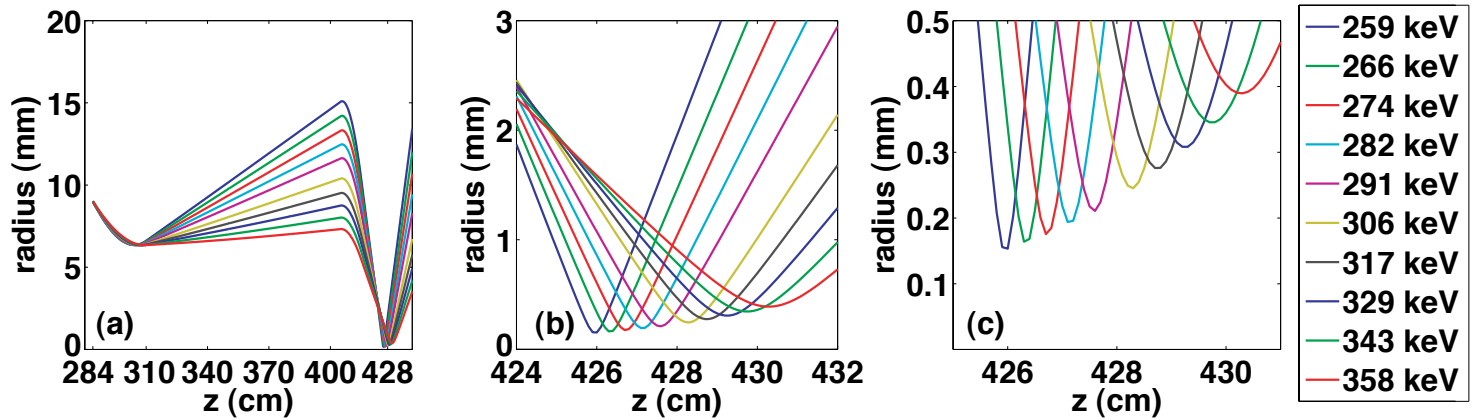

Figure 4.4. Calculated transverse envelopes of different energy slices (a) from the induction gap to the focal plane; (b) near the focal plane and; (c) zoomed in to the see all of the beam waists. Energy slices are color coded in legend.

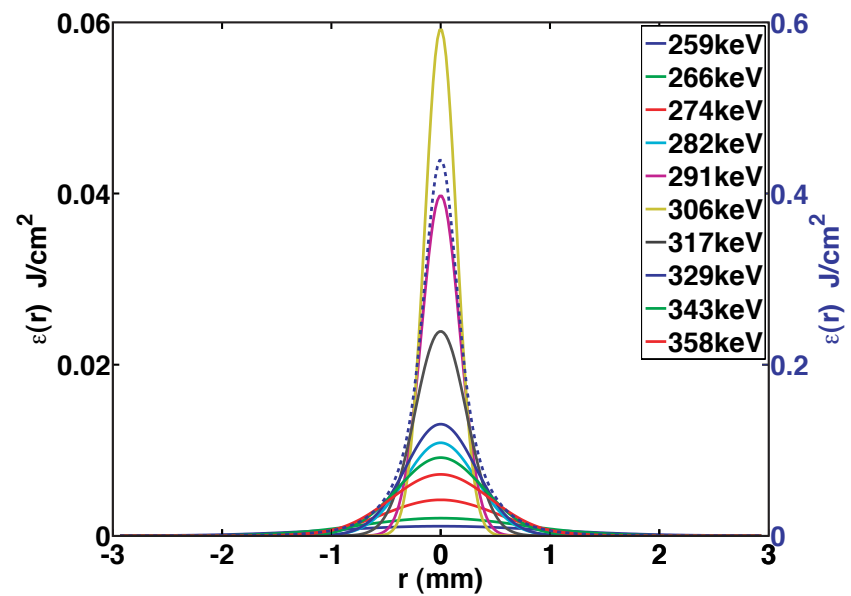

Figure 4.5. Individual Gaussian slices at the focal plane and the composite bunch (dashed blue line). Energy slices are color coded in legend. The left vertical axis is for individual beam bunches and the right vertical axis is for the composite beam bunch. 
in radius of the focused distribution due to chromatic aberrations is also shown for different focusing geometries in Section 6.4.

One disadvantage of these envelope calculations is that they only represent a trace of the beam envelope at the $2 \mathrm{rms}$ excursion, and do not include individual particle motion. Although there is fairly good agreement between these methods and particlein-cell (PIC) techniques, the envelope models are fairly idealized. Particularly the modeling described in the last two sections, which assume perfect neutralization of the beam space charge. We will show in Chapter 6 this may not actually be the case. Additionally, these calculations also do not include other complex effects of nonlinear field effects, centroid offsets and so forth. However, these calculations are very useful because they are extremely quick, do not require a complicated input deck, and provide essential guidance for the design of the experiment under idealized assumptions. Thus, we have an upper bound which is set by these calculations.

\subsection{Particle-in-cell (PIC) codes}

\subsubsection{Warp}

The Warp code is a multi-dimensional PIC code that was developed to study the propagation of space charge dominated beams throughout focusing lattices and accelerators. More detailed descriptions of the code are found in Refs. [91-94]. The Warp code combines the PIC simulation technique, which is time-dependent, with the actual elements providing the applied fields in the accelerator lattice. The code's accelerator lattice consists of accelerating, focusing, and bending elements, in addition to elements with arbitrarily applied fields, all of which have the freedom to overlap. The PIC model includes space-charge effects; these self-fields are assumed to 


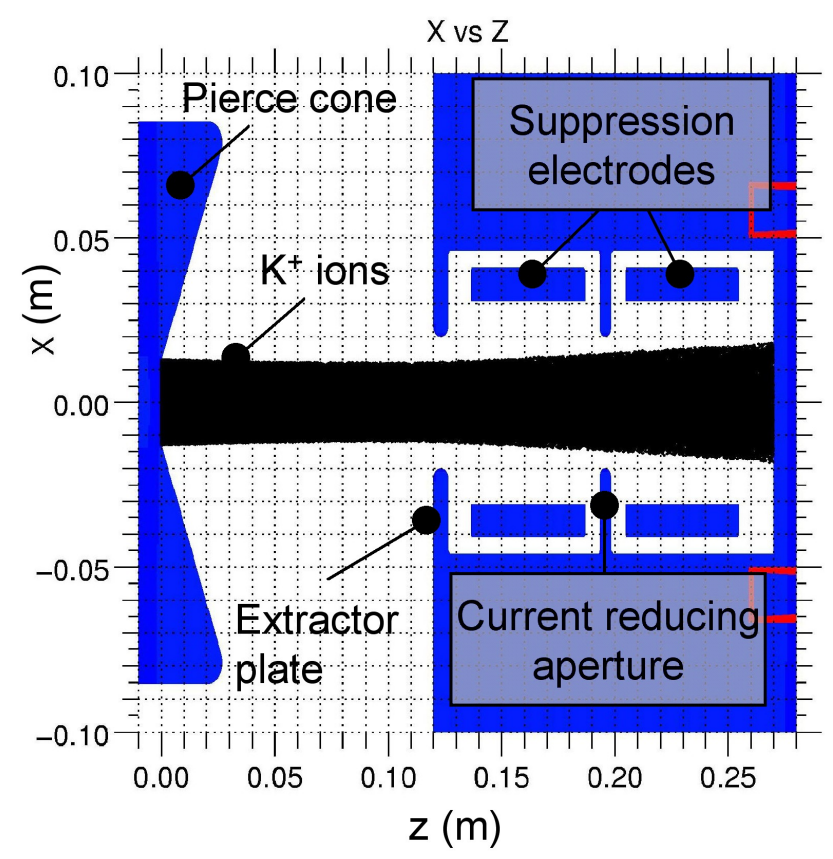

Figure 4.6. Warp3d simulation of the NDCX diode geometry and suppression electrodes with removable aperture downstream.

be electrostatic and are calculated on a Cartesian grid, which moves with the particle distribution as it propagates throughout the accelerator lattice. The code uses the Lorentz force law to advance the beam particles in space and time.

The Warp code has the ability to model particle distributions three ways and all in parallel. Warp3d models the beam in full three-dimensional physical space and threedimensional velocity space (6-D phase space). Another method (Warprz) models the beam assuming axisymmetry. Particles are still followed in full 6-D phase space, but particle density and self-fields are mapped in the $\mathrm{r}-\mathrm{z}$ plane with no variation along the azimuth. The final method (Warpxy) uses a transverse slice model to follow the beam through the accelerator lattice. Since a thin transverse slice of the beam is modeled the z-dependence of the self-fields is ignored. Each of these three methods were used to model the beam dynamics on the NDCX. A Warp3d simulation of the NDCX diode geometry is shown in Fig. 4.6. 


\section{Electron cloud effects model}

Warp was also upgraded to model electron cloud effects due to beam interactions with a material in its path $[59,96]$. These interactions cause gas desorption, collisional ionization, and release of electrons from the material the beam is incident upon. Several different software packages and algorithms were added to model these effects. There is a module for gas desorption [98], while impact ionization is handled by the TxPhysics library [97], and the production of primary and secondary electrons is provided by the POSINST electron-cloud package [44].

In addition Warp was upgraded with an algorithm that models the electron motion with larger time steps, achieved by ignoring the electron gyrofrequency [99]. In

addition, the Chombo mesh-refinement code [100] is incorporated into WARP. Additionally, in the last few months details were added to model neutralization of the beam space charge with a background plasma. Details of the capabilites of Warp modeling and reproducing experimental results are shown in Chapters 5 and 7 .

\subsubsection{LSP}

LSP is a 3-D PIC code which is designed to model large scale plasmas (where it derives its name) [101]. The code models systems with Cartesian, cylindrical, or spherical coordinates in addition to 2-D and 1-D geometries. LSP is an electromagnetic code that calculates applied and self-generated electric and magnetic fields in addition to the interactions between charged particles.

A vast suite of algorithms exist for modeling. A direct-implicit electromagnetic algorithm is used with energy-conserving particle advance. This maintains relatively low plasma temperatures, avoiding the usual problems of numerical heating on the computational grid for small time steps, $\Delta \mathrm{t}<1 / \omega_{\mathrm{p}}[102,103]$. A non-relativistic 
inertial fluid model also exists for electrons, in which the directed and thermal energy of the electrons are treated separately. This allows electrons in dense plasmas to be modeled with a kinetic or fluid treatment as appropriate, eliminating numerical cooling for large time steps, $\Delta t>1 / \omega_{\mathrm{p}}$. The combination of these two algorithms reduces the the usual limitations on time step.

For large simulations, a plasma model which incorporates Ohm's Law, $\mathrm{J}=\sigma(\mathrm{E}$ $+\mathrm{v}_{\mathrm{p}} \times \mathrm{B}$ ), is used, where $\sigma$ is the scalar conductivity and $\mathrm{v}_{\mathrm{p}}$ is the plasma electron velocity. This model reduces computation time and provides a fairly accurate calculation of the neutralization of the beam space-charge by a background plasma $[71,104]$.

Additional algorithms are also available for desorption of neutrals from surfaces, ionization of neutrals, secondary electron generation, field emission, multiple scattering, surface heating, dielectrics, dispersive magnetic materials, and transmission-line boundaries. With all of these tools at hand, a fully-integrated source to target simulation is feasible with LSP [104] as shown in Fig. 4.7. Details of the capabilities of LSP modeling and reproducing experimental results are shown in Chapters 6 and 7 . 


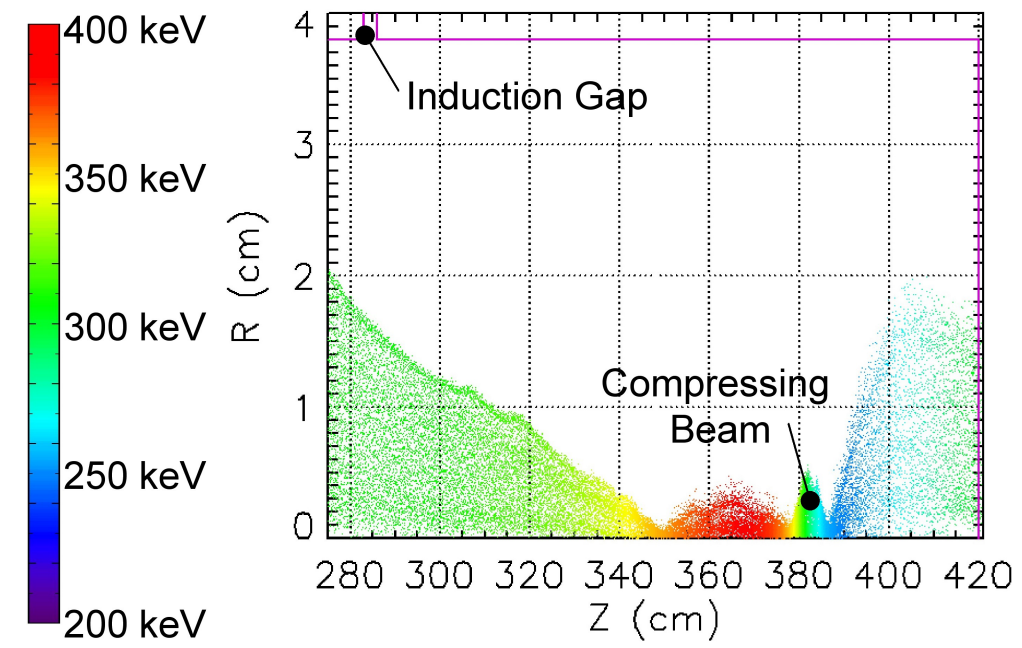

Figure 4.7. Integrated simulation of a neutralized compressing $300 \mathrm{keV} \mathrm{K}^{+}$ion beam (zoomed into neutralized drift region). The induction gap, energy varaition, and compressing bunch are labeled. 


\section{Chapter 5}

\section{Beam injection, matching, and transport}

A large portion of the material in this chapter is also being published in Physical Review Special Topics - Accelerators and Beams $[12,13]$. Here we present results of beam characterization of a $10 \mu \mathrm{s}$, singly charged $\mathrm{K}^{+}$ion bunch at an ion energy of 0.3 $\mathrm{MeV}$ and currents of 26 and $45 \mathrm{~mA}$ after injection, matching and transport through a solenoid lattice. The impact of electron cloud effects and beam centroid motion on the beam quality and dynamics is also addressed.

\subsection{Ion source and injector}

The $\mathrm{K}^{+}$ions are provided by an alumino-silicate ion source $[111,112]$. Traditionally we use Alkali metals because of their low ionization energy. These ion sources preferentially emit ions at the space charge limit for $\mathrm{T}>1000{ }^{\circ} \mathrm{C}$. This allows low-emittance and high-current ion extraction with negligible gas interaction. Low 


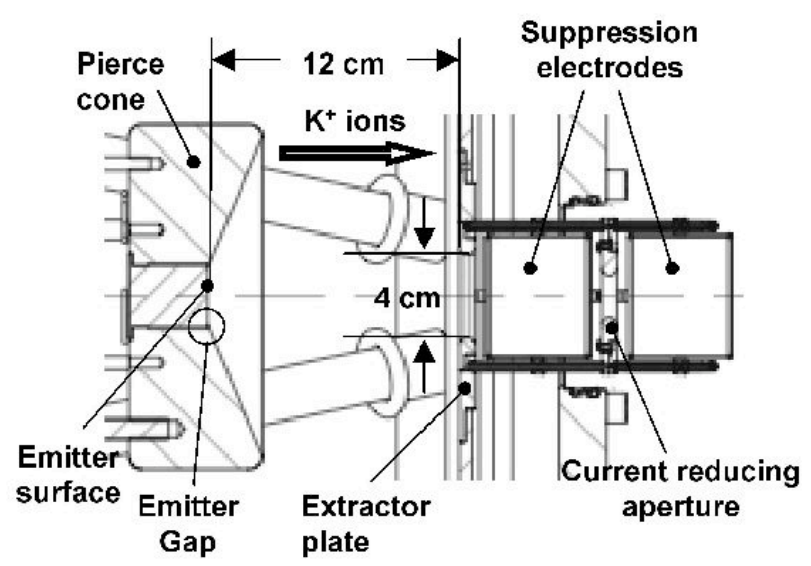

Figure 5.1. Diagram of the Pierce diode geometry and suppression electrodes with removable aperture downstream (Note aperture not installed for these experiments).

emittance is a priority in order to achieve the high intensity necessary for heating targets.

A 2.5-cm diameter ion source was placed in a Pierce electrode and the beam was accelerated through a $12-\mathrm{cm}$ long diode and extracted through a $4-\mathrm{cm}$ diameter aperture (Fig. 5.1) by a $300 \mathrm{kV}, 10-\mu$ s long voltage pulse (Fig. 5.2). The voltage pulse was provided by a $500 \mathrm{kV}$ Marx capacitor bank. Two cylindrical electron suppression electrodes, with a removable current reducing aperture between them, followed directly downstream of the diode. The extracted beam current (45 mA) measured with a $6.35 \mathrm{~cm}$ diameter Faraday cup displayed a relatively flat profile through the pulse duration (Fig. 5.2).

\subsubsection{Beam characterization at the exit of the gun}

Transverse beam dynamics measurements were made to characterize the injected beam between 15 and $31 \mathrm{~cm}$ downstream of the exit of the diode before installing the solenoids (Fig. 5.1). The measurements were made using the two separate diagnostic 


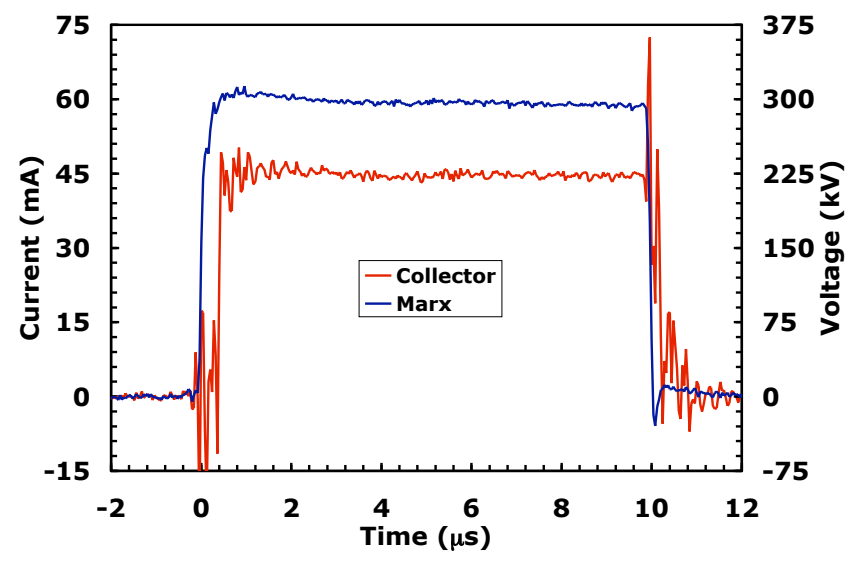

Figure 5.2. Marx voltage waveform used to extract the beam through the diode (blue) and the measured beam current $28 \mathrm{~cm}$ downstream of the diode (red; $\mathrm{z}=40 \mathrm{~cm}$ ).
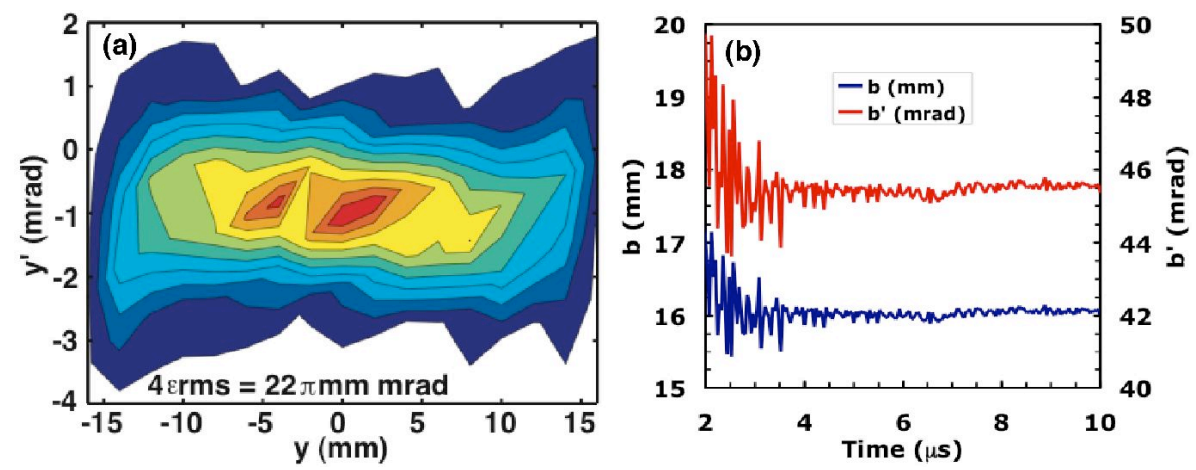

Figure 5.3. (a) Sheared phase space distribution integrated over a 500 ns gate in the middle of the $10 \mu \mathrm{s}$ beam pulse; (b) beam envelope as a function of time $15 \mathrm{~cm}$ downstream of the exit of the extractor $(\mathrm{z}=27 \mathrm{~cm}$; no solenoids).

techniques described in Section 3.2. The measured distribution shown in Fig. 5.3 was made using the double slit and Faraday collector technique. The distribution was nearly uniform and axisymmetric, although it had an angle offset of 1 mrad relative to the desired beamline. The measured normalized emittance $\left(4 \epsilon_{\mathrm{nrms}}=0.088 \pi \mathrm{mm}\right.$ mrad) was only a factor of two greater than the calculated thermal emittance for the ion source (Eq. 2.36). The envelope parameters were also constant over the full pulse. 

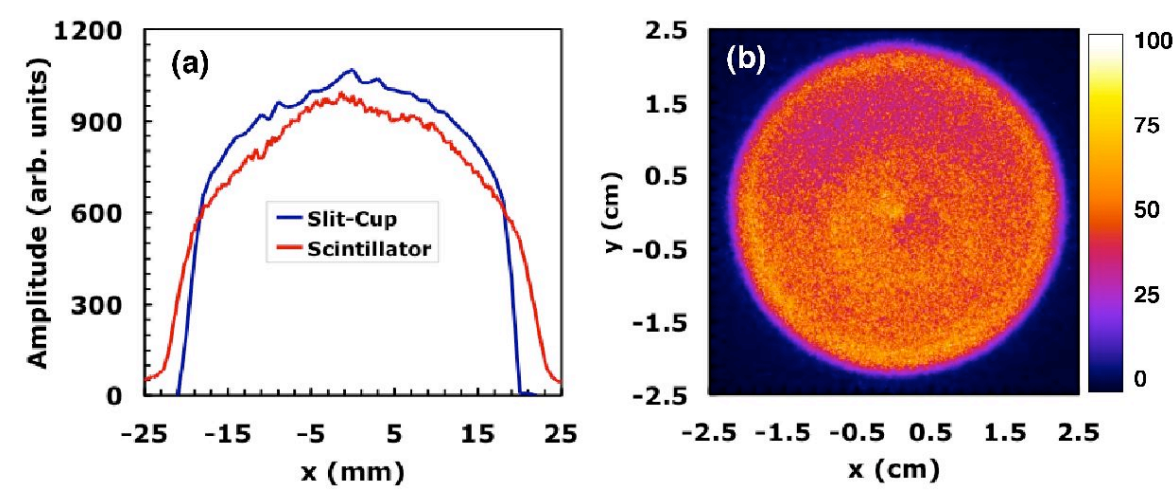

Figure 5.4. Transverse profile of the beam measured: (a) $25 \mathrm{~cm}(\mathrm{z}=37 \mathrm{~cm}$ ) and $31 \mathrm{~cm}(\mathrm{z}=43 \mathrm{~cm})$ downstream of the exit of the extractor. (b) Transverse beam distribution measured at the scintillator plane. All integrated over a 500 ns gate in the middle of the $10 \mu$ s beam pulse (no solenoids).

The transverse beam distribution $[\mathrm{J}(\mathrm{x}, \mathrm{y})]$ and profile were measured optically and with a slit-cup. Due to the $45 \mathrm{mrad}$ divergence angle and the beam space charge, the measured 2rms radius of the beam expanded from $21 \mathrm{~mm}$ to $26 \mathrm{~mm}$ over the 6 $\mathrm{cm}$ drift distance from the slit-cup plane to the scintillator plane (Fig. 5.4). These measurements show a left-right symmetric distribution when projected onto the $\mathrm{x}$ axis, however the distribution in Fig. 5.4(b) has radial contours. There is a slight peak in the center, moving out radially the intensity falls until reaching a higher intensity rim around the edge of the beam, also most of the upper left region of the distribution is below $50 \%$ of the peak intensity. These radial contours in the distribution are a sign of non-uniform focusing fields from the diode.

Particle in the cell simulations using the Warp code [94] were performed to understand how these contours in the distribution are generated. Studies of the Pierce geometry have indicated that the placement of the emitter surface flush to the knife edge of the Pierce cone (Fig. 5.1) is necessary for producing a uniform distribution without radial contours [Fig. 5.5(a)]. In the ideal case the Pierce electrode creates a uniformly distributed set of electric field contours from the surface of the emitter to 

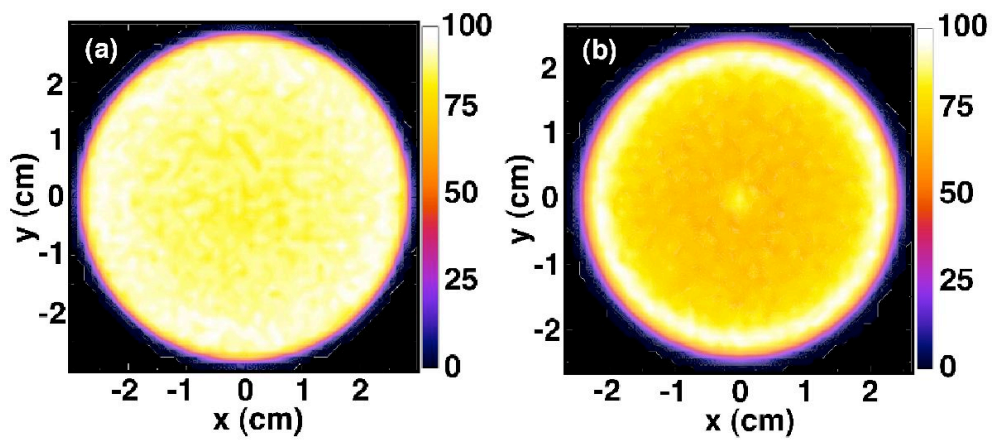

Figure 5.5. Transverse beam distribution calculated in Warp at the scintillator plane $(\mathrm{z}=43 \mathrm{~cm})$ : (a) ideally with no emitter gap; (b) with the emitter surface recessed 22 mils back from the knife edge of the Pierce cone. (no focusing with solenoids; note scale differences).

the exit of the diode. During design and assembly careful attention is made to place the emitter close to the knife edge of the Pierce cone. However, adequate spacing is needed to allow for thermal expansion of the emitter housing during operation. Calculations show that placing the emitter surface 22 mils back from the knife edge of the Pierce cone reproduces the contoured distribution and the measured emittance with relatively good agreement [Fig. 5.5(b)]; there is a rim around the edge of the distribution and a slight peak in the center. This placement of the emitter surface creates a field distortion that accelerates particles at the edge into the beam, leading to the non-uniform distribution measured in Fig. 5.4(b) and calculated in Fig. 5.5(b).

This predicted displacement of the emitter surface from these calculations is relatively small $(<1 \mathrm{~mm})$ and is difficult to verify by measurement while the emitter is under operating conditions $\left(\mathrm{T} \sim 1000{ }^{\circ} \mathrm{C}\right)$ in vacuum. 


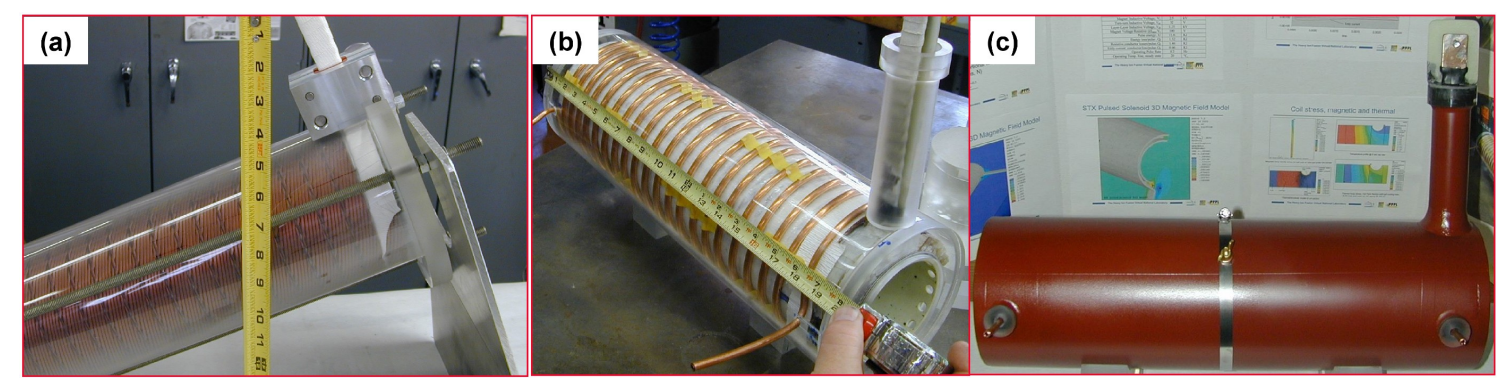

Figure 5.6. Photographs of a pulsed solenoid throughout the construction stage: (a) wound litz cable ready for first potting; (b) copper cooling water tube was added over the potted coil pack; (c) finished solenoid.

\subsection{Pulsed solenoids}

The solenoids used for the NDCX were wound from litz cable consisting of 12 strands with \#10 NEMA-35C film insulating the conductor. The cable was flat and had a rectangular cross section of $0.4 \mathrm{~cm} \mathrm{x} 2 \mathrm{~cm}$. Four layers with 20 turns each were wound on a 9-cm diameter, 4-mm thick NEMA G-10 tube [Fig. 5.6(a)]. The magnets were potted with a layer of CTD-105 epoxy. A copper cooling water tube was added over the potted coil pack [Fig. 5.6(b)] and then the assembly was potted with a heat conducting epoxy. The finished magnets were $50-\mathrm{cm}$ long and had a mean coil radius of $5.75 \mathrm{~cm}$ and coil length of $47 \mathrm{~cm}$ [Fig. 5.6(c)] [14].

High voltage breakdown tests on the conductor showed breakdown voltages from 5 to $15 \mathrm{kV}$. These magnets are capable of producing fields up to $6 \mathrm{~T}$, but the maximum field necessary to overcome the space charge self-field of the beam, obtain the desired envelopes, and to avoid scraping is $\sim 3 \mathrm{~T}$, which requires $12 \mathrm{kA}$ of current. This was provided by a pulse forming network which consisted of a silcon-controlled rectifier (SCR), a power supply, and a SCR switched capacitor bank that yielded a $4 \mathrm{~ms}$ half-sine pulse [14].

The magnetic fields were mapped out in detail using two B-dot probes. The probes 


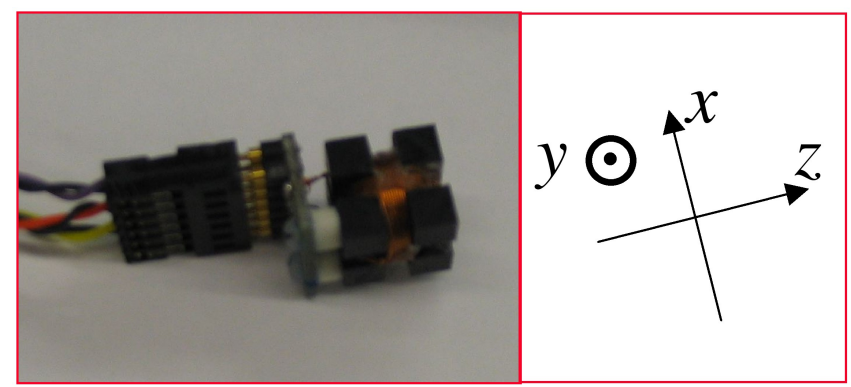

Figure 5.7. Photograph of a three-axis B-dot probe used to map the magnetic fields of the solenoids.

were wound from insulated No. 32 AWG copper wire. Each probe had three-axes (x, $y$, and $z$ ) with 2 layers consisting of 10 turns each and a mean coil diameter of $\approx 12$ mm (Fig. 5.8). One was used to map the axial magnetic field on axis and the other was used to map the axial and radial magnetic fields $3.5 \mathrm{~cm}$ off axis.

Axial magnetic field measurements showed a uniform and symmetric distribution when projected onto the z-axis [Fig. 5.10]. They also agreed well with a simple, thin-coil model using an on-axis fringe function. Eddy currents were also accounted for in the magnetic field measurements by using the $8.5-\mathrm{cm}$ diameter, $1.5-\mathrm{mm}$ thick beampipe, and the 2-cm thick stainless-steel plate used as a flange at the source tank and diagnostic chamber. Measurements and simulations showed that eddy currents only decreased the focusing strength of the magnet by 1 percent [113].

\subsection{Measurements with two solenoids}

Two solenoids were placed on the NDCX beamline immediately downstream of the diode to study the matching and transport of an intense $\mathrm{K}^{+}$ion beam (Fig. 5.9). The focusing lattice consisted of two $50-\mathrm{cm}$ long solenoids spaced about $9 \mathrm{~cm}$ apart with a diagnostic box at the exit of the second solenoid. An additional cylindrical 


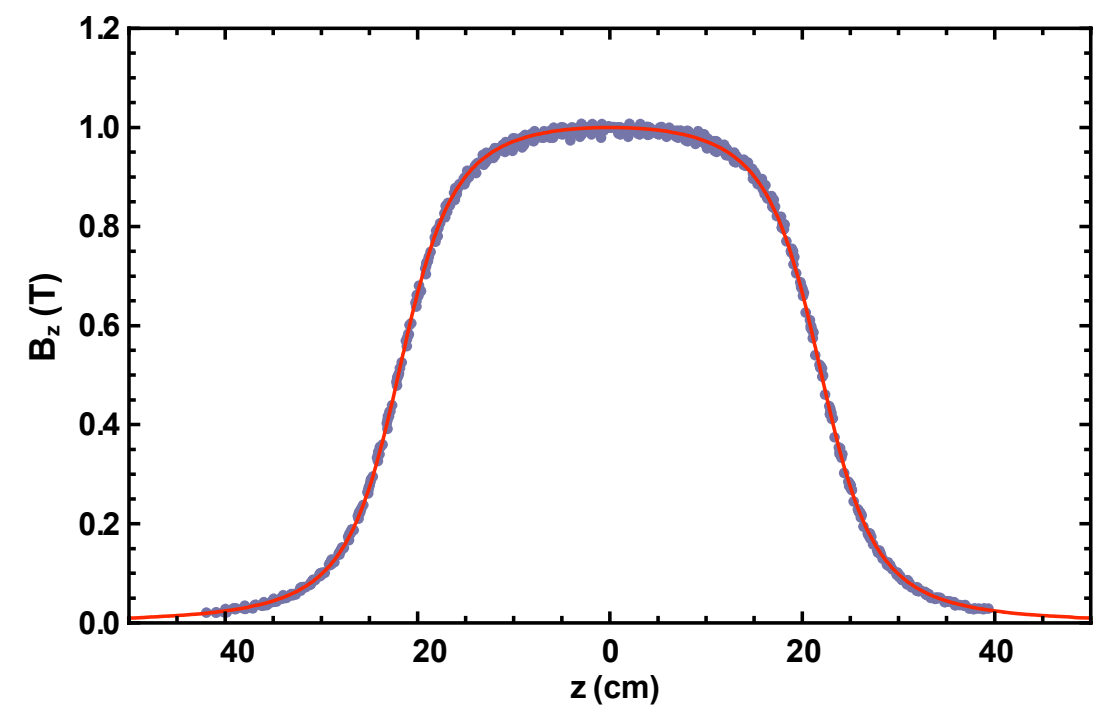

Figure 5.8. Axial magnetic field measurements (blue data points) projected onto the z-axis in comparison to a simple, thin-coil model using an on-axis fringe function (red).

electrode was added at the exit of the last solenoid, upstream of the intercepting diagnostics, to suppress any electrons from backstreaming into the solenoid lattice. The field strengths in Fig. 5.10(a) were chosen to give the matched envelope shown in Fig. 5.10(b). If the extracted beam was ideal (zero emittance and uniform axial velocity), the solenoid field of $2.5 \mathrm{~T}$ used to match the space charge self-field of the beam, $13 \mathrm{~mm}$ in radius, would establish Brillouin-flow at a Larmor frequency $\left(\omega_{\mathrm{c}} / 2\right)$ of $3 \times 10^{6} \mathrm{rad} / \mathrm{sec}$.

Note there is a fringe magnetic field of $0.3 \mathrm{kG}$ at the emitter surface and $1 \mathrm{kG}$ at $\mathrm{z}$ $=142 \mathrm{~cm}$ (upstream slit location), $11 \mathrm{~cm}$ downstream of the exit of two solenoids. The field at the emitter surface can contribute to a small canonical angular momentum that may cause hollowness in the beam distribution after focusing [20]. Calculations show the canonical angular momentum defocusing term is smaller than the emittance defocusing term in the envelope equation (Eq. 2.37) and Warp calculations confirm 


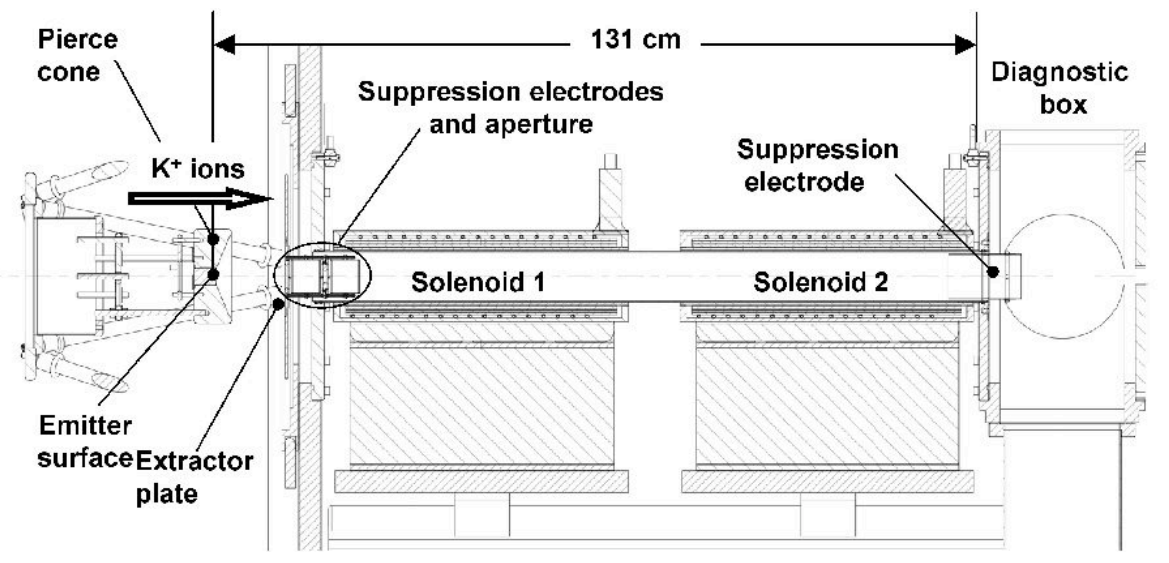

Figure 5.9. Elevation view of the Two-Solenoid Experiment.
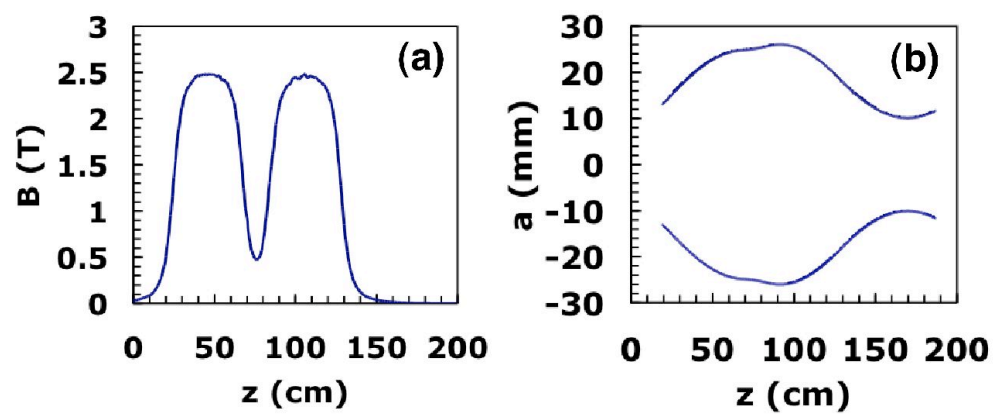

Figure 5.10. (a) Axial magnetic field profile based on measurements; (b) calculated beam envelope. 

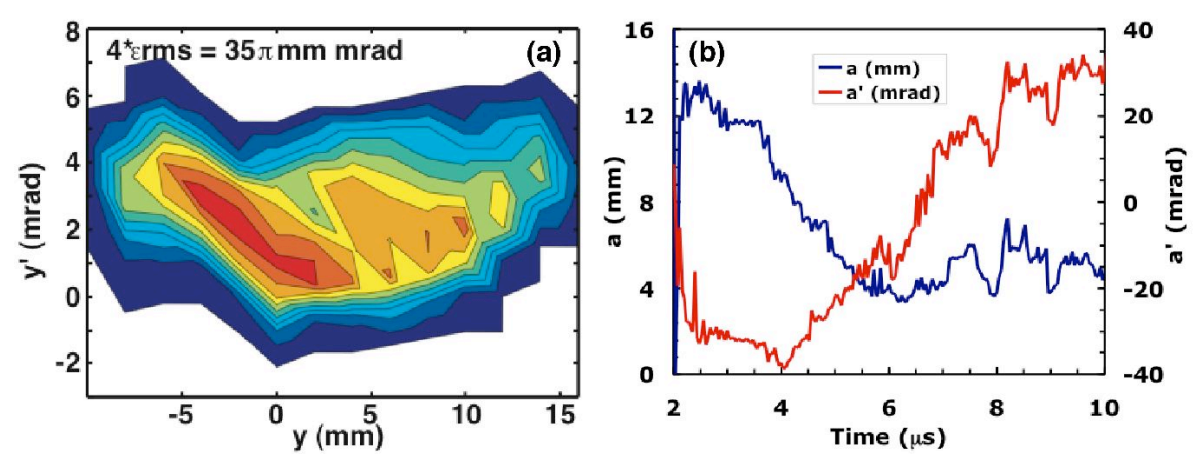

Figure 5.11. (a) Sheared phase space distribution integrated over a 500 ns gate 1 $\mu$ s into beam pulse; (b) beam envelope as a function of time $11 \mathrm{~cm}(\mathrm{z}=142 \mathrm{~cm})$ downstream of the exit of two solenoids.

it is negligible. Details of the effects from the field at the upstream slit location are indicated below.

\subsubsection{Observed electron cloud effects}

Initial measurements of the beam at $\mathrm{z}=142 \mathrm{~cm}, 11 \mathrm{~cm}$ downstream of the exit of the solenoid lattice, showed unexpected emittance growth and time dependence. For example, transverse phase space measurements $1 \mu$ s after the beam head displayed an emittance increase of about a $60 \%$ from that measured at the gun (Figs. $5.3 \&$ 5.11). The emittance continued to rise throughout the pulse and time dependence was observed in the beam envelope (Fig. 5.11).

An unusual signal was also measured on the suppression electrode at the exit of the second solenoid when a slit paddle intercepted the beam. There was a positive capacitive image current at about $1 \mu$ s when the beam entered the diagnostic followed by a rising positive current and then high-frequency oscillations with a period of about 100 ns [Fig. 5.12(a)]. Details of the oscillations were found to be random; every shot showed a somewhat different high frequency pattern. A decrease in the beam radius 

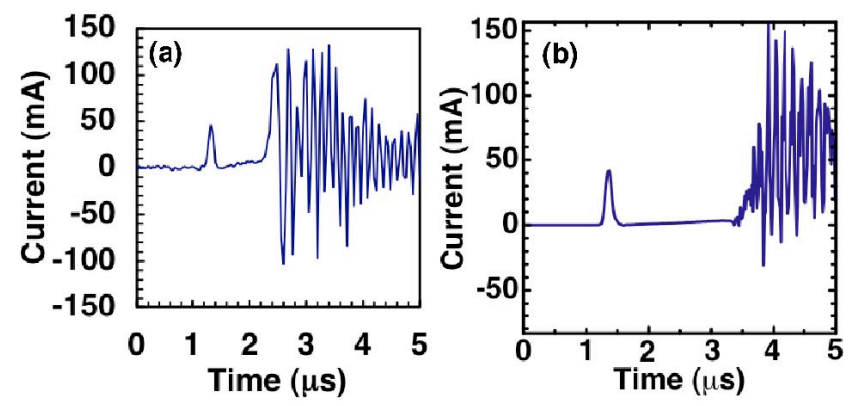

Figure 5.12. (a) Signal measured on the suppression electrode at the exit of the second solenoid with a slit paddle intercepting the beam; (b) current calculated for the same case by a Warp simulation (note scale differences).

and a change in the envelope angle were measured at the onset of these high-frequency oscillations [Fig. 5.11(b)]. Over the 10- $\mu$ s pulse the beam envelope angle switched from a converging angle of $30 \mathrm{mrad}$ to a diverging angle of $30 \mathrm{mrad}$, suggesting that the beam was being neutralized upstream due to backstreaming electrons that were not effectively suppressed by the suppression electrode regardless of the bias voltage.

It is known that intense ion beams will yield electrons from secondary emission and ionization of desorbed gas once the beam is incident upon a surface [50, 55-57]. The slit paddle that intercepts the beam path is composed of stainless steel, which is known to adsorb various gases. In another experiment residual gas measurements during the pressure rise from a $\mathrm{K}^{+}$beam incident on stainless steel show that hydrogen is the main component of the desorbed gas and it expands into the beam path with an average velocity of $0.5 \mathrm{~mm} / \mu \mathrm{s}[58]$.

Particle-in-cell simulations using Warp [94] were conducted to study the interactions of the beam with desorbed gas, and subsequent ions and electrons [59]. These interactions are also relevant to electron cloud studies in other high intensity accelerators [38-47]. The calculated current on the suppression electrode [Fig. 5.12(b)] 
has several features that qualitatively agree with the experimental measurement. A positive capacitive image current appears at about $1 \mu$ s when the beam enters the diagnostic. The calculated time for a positive current to appear on the suppressor due to ionized $\mathrm{H}_{2}$ is about $1 \mu$ s after the head of the beam, consistent with the observations in this experiment. This positive current continues to rise and then is followed by high-frequency oscillations.

The observed high-frequency oscillations on the suppression electrode are due to an oscillating virtual cathode [114]. Once enough desorbed hydrogen gas is ionized the $\mathrm{H}_{2}{ }^{+}$and electron densities immediately in front of the slit plate increase to about the beam density, enough to overcome the suppressor potential. Each species forms a sheath and they oscillate at the electron plasma frequency. This process shields the electrode and pushes electrons through the suppressor and $3 \mu$ s after the head of the beam a sufficient amount of electrons backstream into the solenoid fields to partially neutralize the beam. Beam measurements are consistent with this showing an increase in emittance and a gradual decrease in the beam radius at $4 \mu$ s until a $60 \%$ reduction is reached around $5 \mu$ s and the beam begins to diverge [Fig. 5.11(b)].

Through more detailed observations it was determined that the deviations in the measured envelope parameters throughout the beam pulse only occur when the upstream slit paddle intercepts the beam. When the slit-cup and scintillator diagnostics, 10 and $16 \mathrm{~cm}$ further downstream, were used much weaker time dependence was observed in the beam radius (Fig. 5.13). As noted in Section 5.1.1, this envelope fluctuation was not observed when the beam was diagnosed upstream at the gun without any focusing fields. These observations hint electron confinement by the large fringe magnetic fields $(1-1.3 \mathrm{kG})$ helped contribute to the sheath formation and electron cloud and gas effects described above, confounding the measurement of the intrinsic beam distribution. 

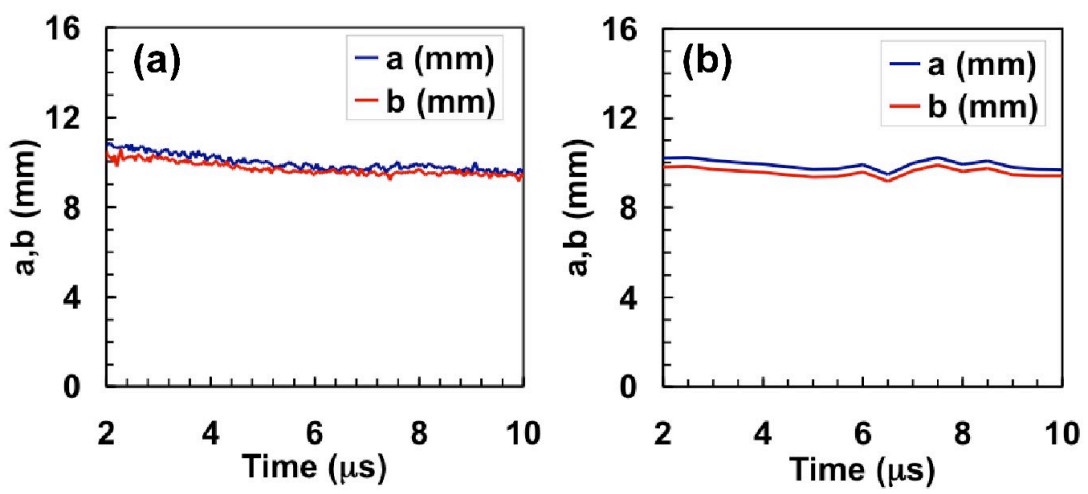

Figure 5.13. Beam radii: (a) $21 \mathrm{~cm}(\mathrm{z}=152 \mathrm{~cm})$ and; (b) $27 \mathrm{~cm}(\mathrm{z}=158 \mathrm{~cm})$ downstream of the exit of two solenoids as a function of time.

\subsubsection{Mitigation of electron cloud effects}

A drift distance of $29 \mathrm{~cm}$ was added between the end of the second solenoid and the suppressing and intercepting diagnostics to test whether measuring the beam distribution with an intercepting diagnostic in a strong magnetic field will confuse the measurement. Each of the diagnostics was now immersed in a field strength nearly an order of magnitude less. This also increased the gyroradii of electrons to several $\mathrm{cm}$, which was on the order of the diameter of the suppression electrodes.

Time dependent measurements of the transverse phase space showed the measured emittance was reduced from previous measurements, and close to the injected beam emittance (22 $\pi \mathrm{mm}$ mrad, Fig. 5.14). These measurements also demonstrated excellent agreement between the two methods of measuring the phase space; double slit and a Faraday collector and optical measurements. The measured beam envelope (at $\mathrm{z}=171 \mathrm{~cm}$ ) also no longer varied drastically in time [Fig. 5.14(c)] and agreed well with the calculated envelope shown in Fig. 5.10(b).

Time resolved measurements of the transverse beam distribution and profile com-

plemented the phase space measurements by also displaying a constant radius versus 

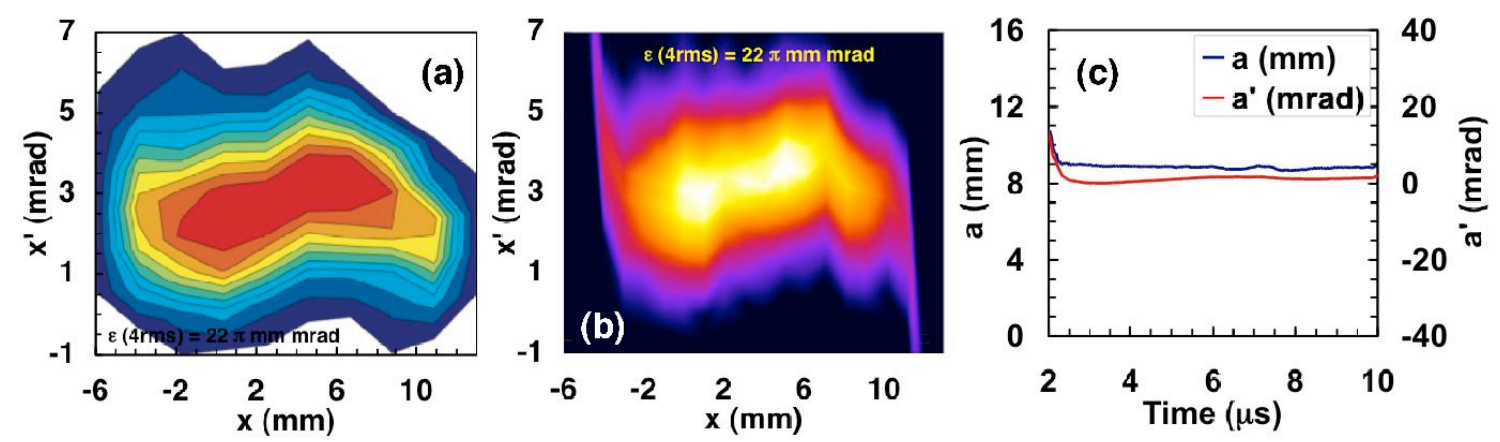

Figure 5.14. Sheared phase space distribution integrated over a 500 ns gate in the middle of the $10 \mu$ s beam pulse: (a) measured with a double slit and a Faraday collector; (b) measured optically and; (c) beam envelope as a function of time. All measured $40 \mathrm{~cm}$ downstream of the exit of two solenoids $(\mathrm{z}=171 \mathrm{~cm})$.
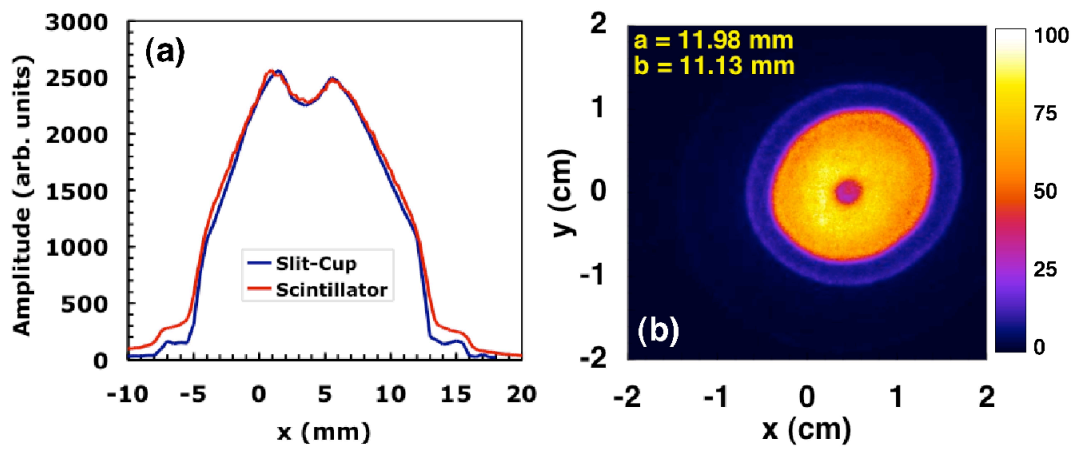

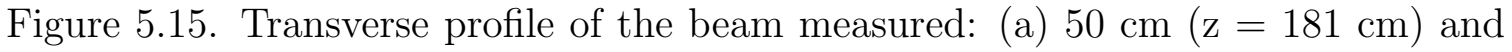
$56 \mathrm{~cm}(\mathrm{z}=187 \mathrm{~cm})$ downstream of the exit of two solenoids. (b) Transverse beam distribution at the scintillator plane. All integrated over a 500 ns gate in the middle of the $10 \mu$ s beam pulse.

time, showing that spurious electron cloud effects due to the intercepting diagnostics were mitigated as required for WDM and fusion applications. Despite the steadystate envelope, the transverse beam distribution and profile were not uniform or axisymmetric (Fig. 5.15). The beam centroid was offset by several millimeters and milliradians, had a hollow center, and a substantial halo. The causes for these undesired effects in the beam distribution were not initially understood and were explored in the Warp code, discussed below. 


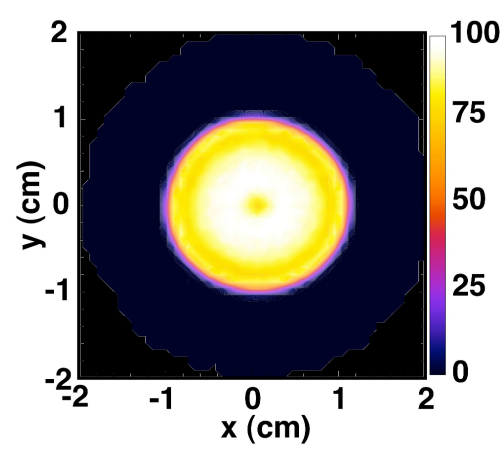

Figure 5.16. Transverse beam distribution calculated $56 \mathrm{~cm}$ downstream of the exit of two solenoids at the scintillator plane $(\mathrm{z}=187 \mathrm{~cm})$ using the recessed emitter.

\subsubsection{Alignment effects on beam dynamics}

Particle in the cell simulations with the Warp code [94] were used to quantify and reproduce the features of the distribution measured at the exit of the transport lattice. As shown in Section 5.1.1 recessing the emitter surface 22 mils back from the knife edge of the Pierce cone reproduces the contoured distribution with relatively good agreement; there is a rim around the edge of the distribution, and the slight peak in the center [Fig. 5.5(b)]. This placement of the emitter surface creates a field distortion that accelerates particles at the edge into the beam leading to the sheared distribution measured in Fig. 5.4(b) and calculated in Fig. 5.5(b).

Calculations with Warp indicate that transporting this sheared distribution through a perfectly aligned solenoid lattice reproduces some of the features in the measured distribution in Fig. 5.15(b). Using the recessed emitter alone, reproduced the radial contours and the density depression in the center of the distribution (Fig. 5.16), however the distribution was still well centered and axisymmetric.

The centroid offsets of the beam distribution are only reproduced after adding solenoid misalignments to the Warp calculation [Fig. 5.17(a)]. It is known from mapping the fields of the solenoids that there are $\geq 1 \mathrm{~mm}$ offsets of the coils within the 

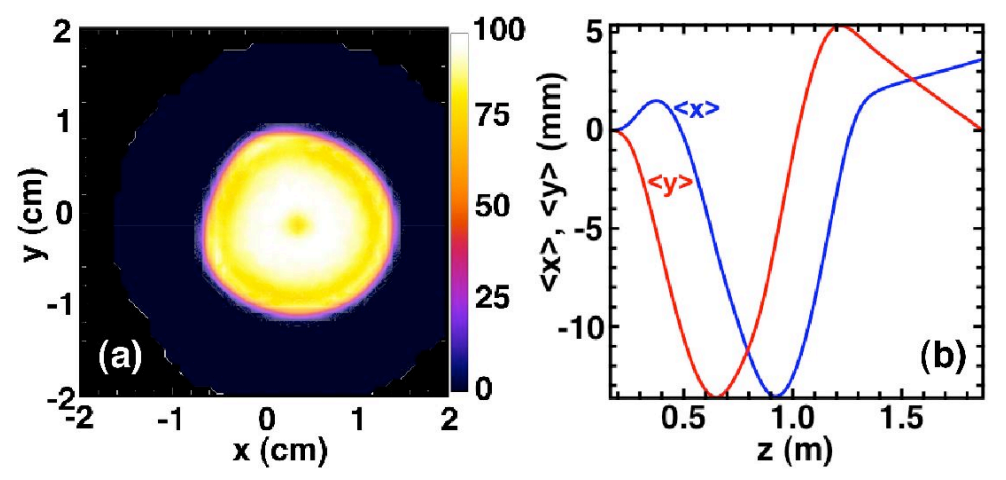

Figure 5.17. (a) Transverse beam distribution calculated $56 \mathrm{~cm}$ downstream of the exit of two solenoids at the scintillator plane $(\mathrm{z}=187 \mathrm{~cm})$ using the recessed emitter and misaligned solenoids; (b) beam centroid offsets along the axis of propagation.

magnet structure. Precisely adding the actual solenoid misalignments is a difficult task, which requires a detailed set of data. Each solenoid has four individual displacements $\left(\langle\mathrm{x}\rangle,\left\langle\mathrm{x}^{\prime}\right\rangle,\langle\mathrm{y}\rangle\right.$, and $\left.\left\langle\mathrm{y}^{\prime}\right\rangle\right)$, two position and two angular displacements relative to the ideal beam axis.

A measurement of the 4-D phase space of the beam provided four individual centroid offsets $\left(\langle\mathrm{x}\rangle,\left\langle\mathrm{x}^{\prime}\right\rangle,\langle\mathrm{y}\rangle\right.$, and $\left.\left\langle\mathrm{y}^{\prime}\right\rangle\right)$ that were inverted to solve for the $\mathrm{x}$ and $\mathrm{y}$ position displacements of the two solenoids only (Table 5.1). This approximation was made to determine the impact of the solenoid position offsets on the beam distribution. The solenoid displacements determined from this inversion were quite large due to the simplicity of the model (i.e. it excluded angular offsets and initial offsets at the emitter $)$. It has been determined analytically that small angular displacements $(<5$ mrad) of the solenoids can contribute to equal or greater beam centroid offsets when compared with solenoid position displacements of $<3 \mathrm{~mm}$ (Section 5.6). A position offset of $2.5 \mathrm{~mm}$ is equivalent to an angular displacement of $10 \mathrm{mrad}$ about the center of a 50-cm long solenoid.

Nevertheless, these calculations reproduced the measured centroid offsets of beam in Figs. 5.14 \& 5.15 reasonably well [Fig. 5.17(a)] and maintain the contoured 
Table 5.1. Approximation of $\mathrm{x}$ and $\mathrm{y}$ displacements of the two solenoids relative to the ideal beam axis.

\begin{tabular}{|c|c|c|}
\hline & S1 & S2 \\
\hline$\Delta \mathbf{x}(\mathbf{m m})$ & -15.88 & -0.77 \\
\hline$\Delta \mathbf{y}(\mathbf{m m})$ & -9.90 & -15.80 \\
\hline
\end{tabular}

distribution with the density depression in the center. The resulting betatron motion of the beam centroid due to the solenoid offsets is shown in Fig. 5.17(b).

The same effect could be demonstrated by offsetting the beam injected into the solenoid lattice. However, from the measurements in Section 5.1.1 it is evident that the extracted beam is well aligned. It would take an offset comparable to the solenoid offsets to demonstrate the observed centroid offsets.

We have been able to produce a distribution with a density depression in the center, higher intensity rim around the edge, and centroid offsets by adding a recessed emitter and solenoid offsets to the Warp calculation. However, the calculated distribution in Fig. 5.17(a) does not display the non-axisymmetric or elliptical shape measured in Fig. 5.15(b). Since the beam is suffering from betatron motion of its centroid it is sampling a greater portion of the nonlinear fields than a perfectly centered beam distribution. This will affect the shape of the beam distribution and it must be included in the calculation.

The fringe components of a solenoid are usually the most significant contributor to nonlinear focusing effects. Consider a finite length current sheet wrapped around the z-axis (in the azimuthal direction). This current sheet acts as an ideal solenoid creating an ideal magnetic field which is uniform except near the ends where the $\mathrm{B}_{\mathrm{z}}$ field is mainly linear versus $\mathrm{r}$. The higher order terms in the fringe are the largest contributors to nonlinear focusing.

After adding the nonlinear field terms into the calculation a better qualitative 

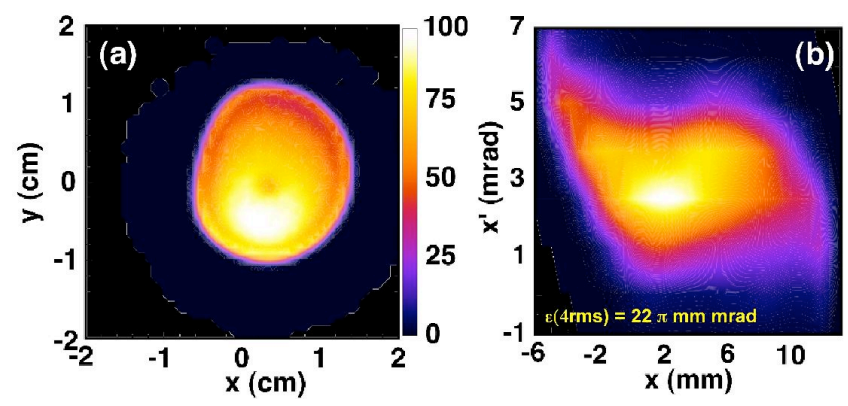

Figure 5.18. (a) Transverse beam distribution calculated $56 \mathrm{~cm}$ downstream of the exit of two solenoids at the scintillator plane $(\mathrm{z}=187 \mathrm{~cm})$; (b) Sheared phase space distribution calculated $40 \mathrm{~cm}$ downstream of the exit of two solenoids at the scintillator plane $(\mathrm{z}=171 \mathrm{~cm})$. All calculated using the recessed emitter, misaligned solenoids, and nonlinear focusing terms.

agreement is observed between the measured distribution in Fig. 5.15(b) and the calculation [Fig. 5.18(a)]. An elliptical shape is observed in addition to a density peak in the lower left of the distribution. The sheared phase space and emittance calculated in Fig. 5.18(b) (22 $\pi \mathrm{mm}$ mrad) is also in good qualitative agreement with those measured in Figs. 5.14(a) \& (b). The centroid offsets are identical by calculation and the distortions at the edge have also been reproduced.

Although the details of the distributions were not exactly reproduced, the factors that contributed to the features seen in the distribution are understood. The recessed emitter causes a non-uniform distribution to be injected into the solenoid lattice and the misaligned solenoids cause a centroid offset to evolve. The shift in the charge distribution [Fig. 5.15(b) \& Fig. 5.18(a)] is due to nonlinear focusing of the beam. Insufficient data were available to solve for the correct solenoid position and angular offsets, and a restricted model yielded unphysically large displacements and angles. A more accurate model and study of the beam centroid motion is presented in Section 5.6 . 


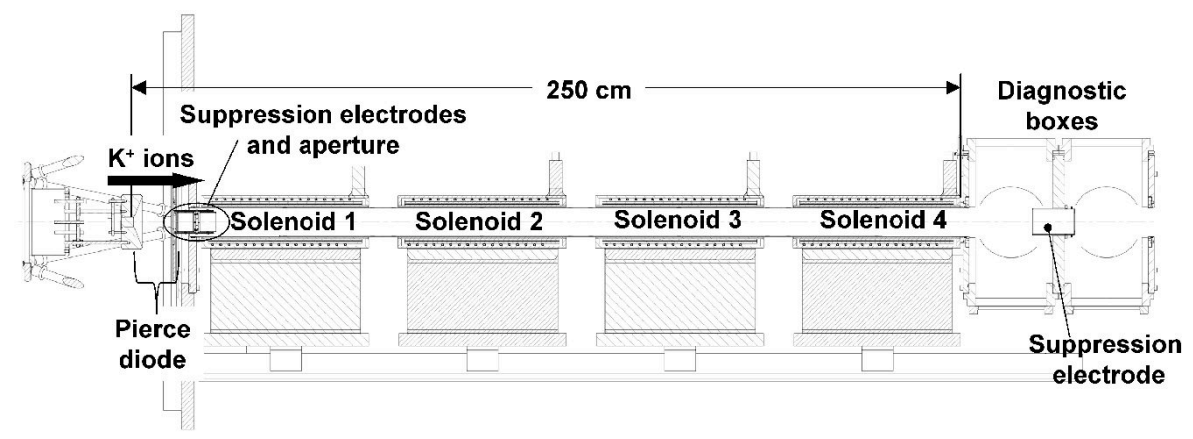

Figure 5.19. Elevation view of the Four-Solenoid Experiment.

\subsection{Electron cloud studies in four solenoids}

The observation of electron cloud effects in experiments with two solenoids presented an opportunity to study these effects in more detail. Two more solenoids were added to the already existing two-solenoid lattice on the NDCX beamline (Fig. 5.19). The two additional solenoids were also 50-cm long, had an identical construction to those used in the two-solenoid experiments, and the spacing in between all of the magnets was about $9 \mathrm{~cm}[12,14]$.

Large fringe magnetic fields, which aided electron confinement and contributed to the sheath formation and electron cloud effects in degrading the beam quality when intercepting the beam in the two-solenoid experiment, were accounted for in this four-solenoid lattice. So the intercepting diagnostics were placed nearly $40 \mathrm{~cm}$ downstream of the exit of the four-solenoid lattice where the axial field was $\sim 100 \mathrm{G}$ or negligible. 

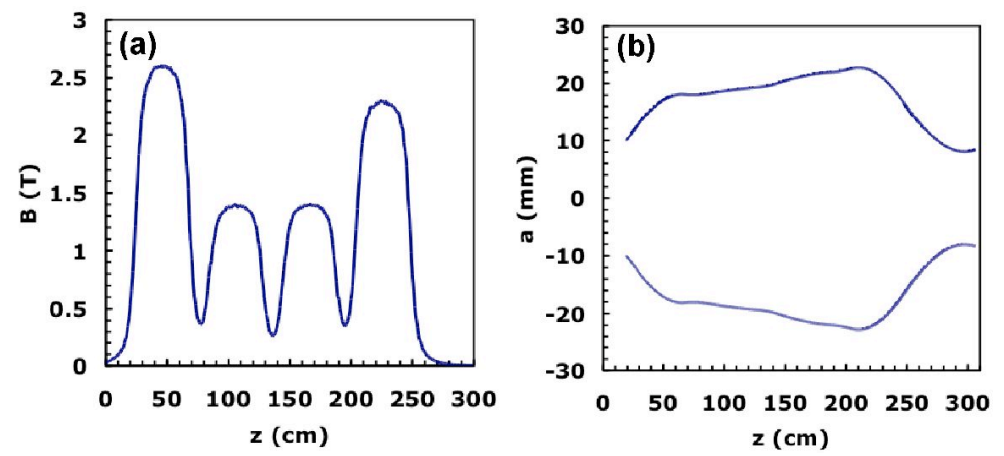

Figure 5.20. (a) Axial magnetic field profile based on measurements; (b) calculated envelope for the apertured 26-mA beam.

\subsubsection{Apertured beam measurements}

These experiments were conducted with a $10-\mu$ s, singly charged $\mathrm{K}^{+}$ion bunch at an ion energy of $0.3 \mathrm{MeV}$. Initial tests with the four-solenoid lattice were done with a current-reducing aperture that reduced the $45-\mathrm{mA}$ beam to $26 \mathrm{~mA}$. The focusing lattice in Fig. 5.20(a) was chosen to give the desired envelope for the 26-mA beam in Fig. 5.20(b). Despite diagnosing the beam in a low ( $100 \mathrm{G})$ magnetic field the measured emittance for the apertured beam was $80 \%$ larger than what was measured directly downstream of the gun without any solenoid focusing (Fig. 5.21). The beam envelope also had slight time dependence but the envelope remained converging and the variation in radius was less than $2 \mathrm{~mm}$ [Fig. 5.21(c)]. The observed time dependence hints there might be partial neutralization of the beam due to electrons which also contributes to the measured emittance increase.

The waveforms in Fig. 5.21(c) are calculated from signal levels $\sim 10$ millivolts which have a low $\mathrm{S} / \mathrm{N}$. The spikes just after $3 \mu$ s and just before $13 \mu$ s are due to image currents from the head and tail of the beam pulse. The spike after $10 \mu$ s corresponds to electrical noise from the crowbar spark gap firing to terminate the Marx voltage pulse at $10 \mu \mathrm{s}$ (Fig. 5.2). 

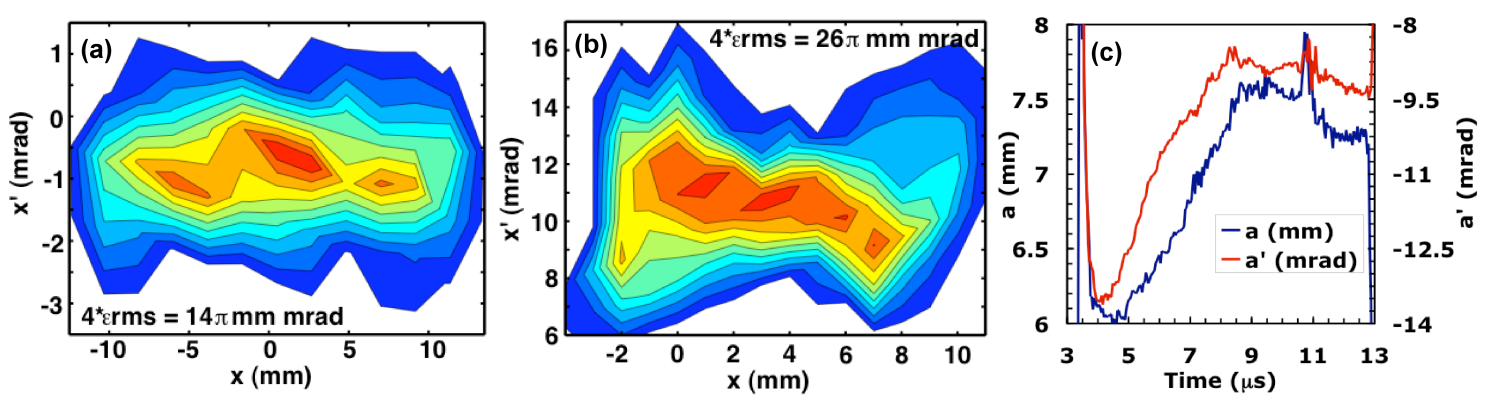

Figure 5.21. Sheared phase space distribution integrated over a $500 \mathrm{~ns}$ gate in the middle of the $10 \mu \mathrm{s}$ beam pulse: (a) $15 \mathrm{~cm}$ downstream of the exit of the extractor $(\mathrm{z}=$ $27 \mathrm{~cm}$; without solenoid focusing); (b) $40 \mathrm{~cm}$ downstream of the exit of four solenoids ( $\mathrm{z}=290 \mathrm{~cm}$; note scale differences) and; (c) beam envelope $40 \mathrm{~cm}$ downstream of the exit of four solenoids as a function of time $(\mathrm{z}=290 \mathrm{~cm})$.

It is known from past experiments without any solenoid focusing and with magnetic quadrupole focusing once the beam strikes the current-reducing aperture, unwanted gas can be desorbed and ionized adding unwanted electrons and ions into the diode and focusing lattice [115]. That is why there are two cylindrical electrodes, each on either side of the aperture biased at $-3 \mathrm{kV}$ to effectively suppress electrons. As stated above the large fringe magnetic fields from the solenoids present difficulties in suppressing electrons particularly when the beam is normally incident upon an object, like the aperture. These observations indicated the electrodes surrounding the aperture might not be suppressing electrons effectively. In the next two sections measurements with electron cloud diagnostics will show how these effects can be mitigated.

\subsubsection{Apertured beam measurements with electron cloud di- agnostics}

Extensive studies of electron cloud and gas effects in the four-solenoid lattice were done with the apertured 26-mA beam using new cylindrical electrodes inside the 


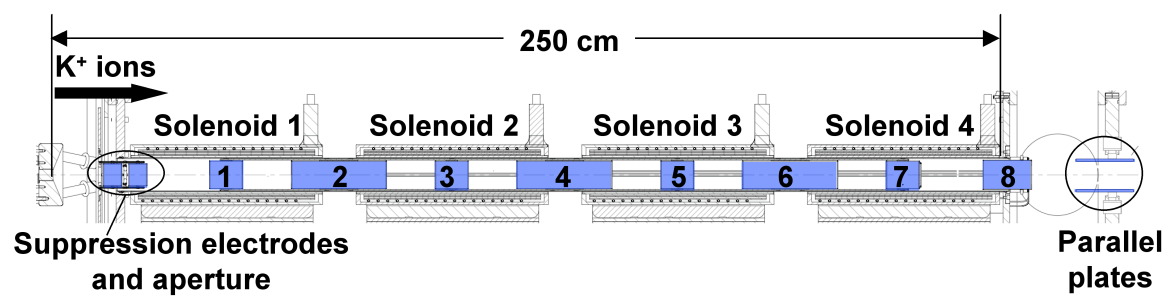

Figure 5.22. Layout of the aperture and suppression electrodes, electron cloud diagnostics: solenoid electrodes (1, 3, 5, and 7), gap electrodes (2, 4, 6, and 8); and parallel plate diagnostic relative to the four-solenoid lattice. All the diagnostics have cylindrical symmetry except for the parallel plate diagnostic.

beam pipe (electron cloud diagnostics; Fig. 5.22). These electron cloud diagnostics (described in Section 3.4.1) are short compared to the beam bunch length $(\sim 1.2 \times$ $\left.10^{3} \mathrm{~cm}\right)$.

These cylindrical electrodes measured a positive capacitive image current of the beam as it entered the diagnostic and negative capacitive image current of the beam as it exited (Fig. 5.23). These capacitive signals are proportional to the derivative of the beam current. The signals are displaced in time due to the time of flight of the beam to each electrode. The width of the spikes alternated between narrow and wider, corresponding to the short solenoid electrodes $(1,3,5$, and 7) and the longer gap electrodes $(2,4,6$, and 8$)$. Measurements showed a growth in the peak of the positive capacitive signal as the beam propagated axially due to overtaking in the beam head as seen in Fig. 3.4. The electrodes collected charge throughout the pulse depending on the bias configuration and location of the diagnostic along the focusing lattice.

These electrodes were independently biased between $\pm 1 \mathrm{kV}$. The solenoid electrodes $(1,3,5$, and 7$)$ were biased negatively to repel electrons, while the gap electrodes $(2,4,6$, and 8$)$ were biased positively to clear electrons from intercepted field lines and suppress emission. Reversing the biases trapped electrons that were emit- 


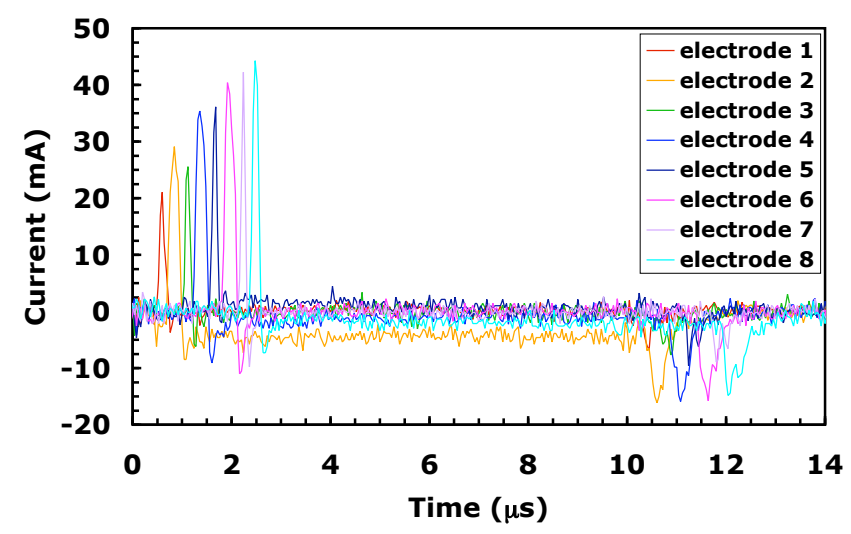

Figure 5.23. Electron cloud diagnostic signals as a function of time.

ted from the gap electrodes between magnets. Results of operating the diagnostics to clear electrons from the lattice showed charge collection began to saturate for voltage biases $|V| \geq 600 \mathrm{~V}$ (Fig. 5.24).

The data points for the charge collected on each of the 8 electrodes were provided by three or more consecutive shots at each bias voltage. This demonstrates the shot to shot variation at each voltage and a general trend for each electrode. Electrodes 1 and 5 were biased negatively to repel electrons, the positive charge collected on electrode 5 is most likely stray ions from gas ionized in the lattice by the beam ions or electrons with sufficient energy.

Some of the evidence that the electrodes surrounding the aperture were not sufficiently suppressing electrons was seen from the charge collected on the most upstream gap electrode 2 (Fig. 5.25). Electrons were collected on this electrode regardless of the bias voltage and the threshold was reached just above $+100 \mathrm{~V}$. This electrode was magnetically connected to the aperture, because most of the field-lines from the first solenoid intersected this electrode and the electrode directly downstream of the aperture. Electrons of all energy ranges made by beam and gas interactions in the 

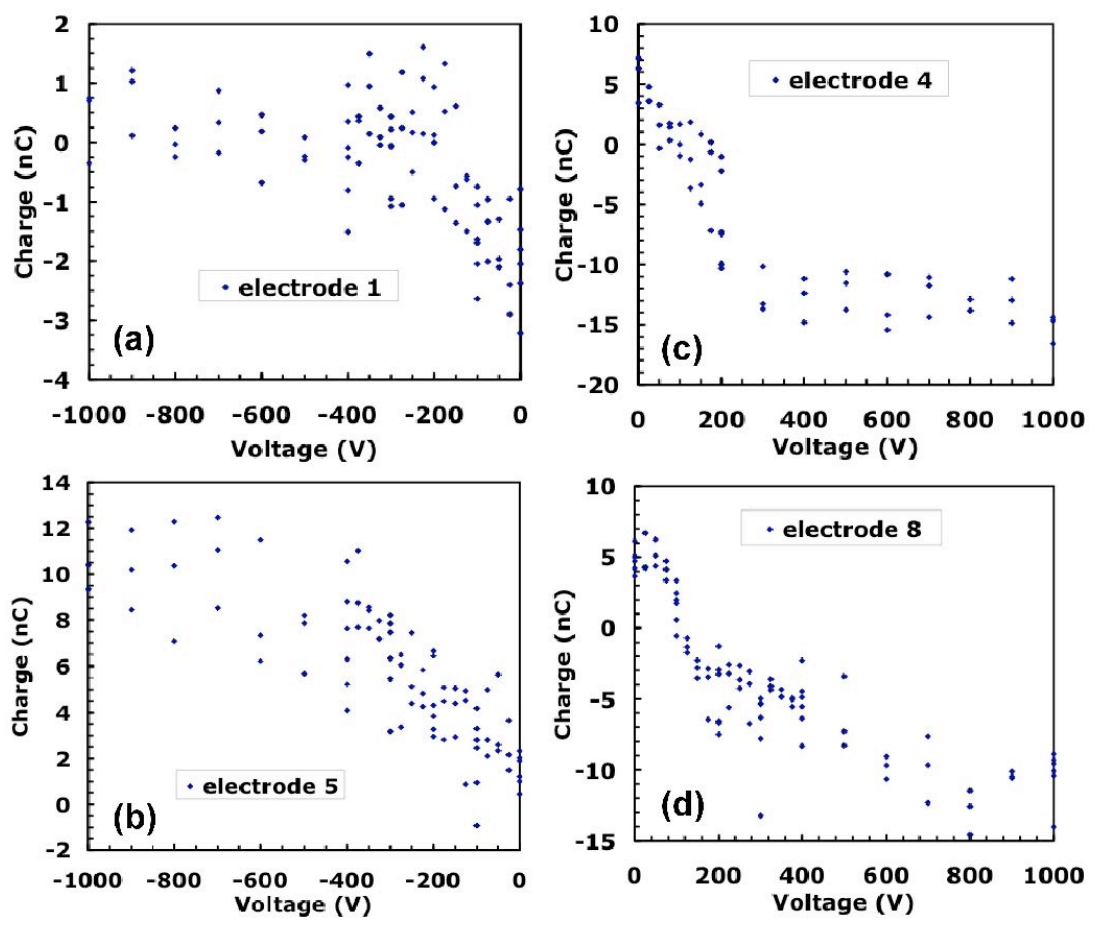

Figure 5.24. Charge collected as a function of bias voltage for: (a) electrode 1; (b) electrode 5; (c) electrode 4; (d) electrode 8. 


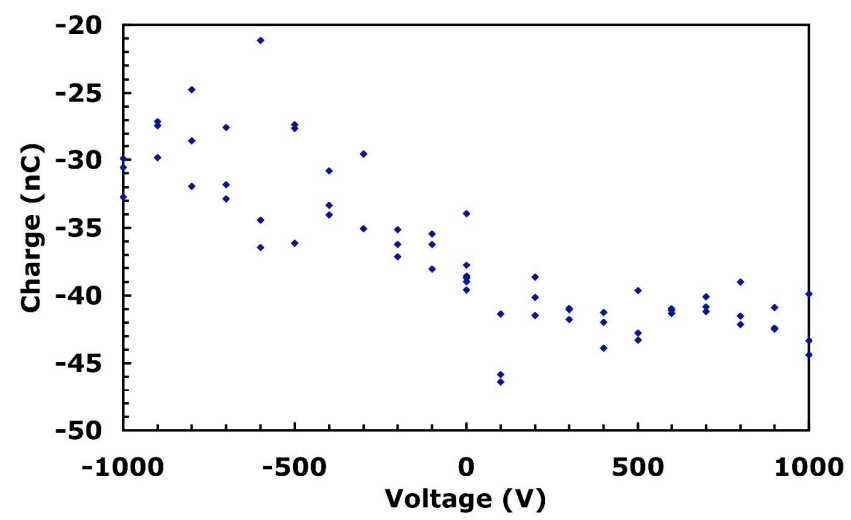

Figure 5.25. Charge collected as a function of bias voltage for electrode 2 .

vicinity of the aperture, which had a flux density $>5 \mathrm{kG}$, were tightly tied with $\mathrm{r}_{\mathrm{ce}}$ $<1 \mathrm{~mm}$ to these fieldlines and collected on electrode 2 .

The amount of current (charge/pulse length) collected on electrode 2 (Fig. 5.23) was used to quantify the line charge density of electrons $\left(\lambda_{\mathrm{e}}=\mathrm{I}_{\mathrm{e}} / \mathrm{v}_{\mathrm{e}}\right)$ relative to the beam line charge density $\left(\lambda_{\mathrm{b}}\right)$ and provide an estimate of the electron density in the solenoid lattice contributed by the beam and gas interactions at the aperture. Assuming the electrons collected on electrode 2 have kinetic energy ( $4 \mathrm{keV})$ provided by the potential difference from the suppression electrode at the aperture to electrode 2 , then $\lambda_{\mathrm{e}}=107 \mathrm{pC} / \mathrm{m}$. Including the electron current from electrodes 4 and 8 increases $\lambda_{\mathrm{e}}$ to $200 \mathrm{pC} / \mathrm{m}$. This is $\sim 1 \%$ of $\lambda_{\mathrm{b}}(21 \mathrm{nC} / \mathrm{m})$ and suggests an electron density, $\mathrm{n}_{\mathrm{e}} \sim 10^{6} \mathrm{~cm}^{-3}, 1 \%$ of the beam density $\left(\mathrm{n}_{\mathrm{K}+} \sim 10^{8} \mathrm{~cm}^{-3}\right)$ might be present in the solenoid lattice. If the electron energies are only few hundred $\mathrm{eV} \mathrm{n}_{\mathrm{e}}$ could be $\sim 6 \%$ of $\mathrm{n}_{\mathrm{K}+}$.

The impact of clearing electrons on the beam quality was evident from optical measurements of the transverse beam distribution $[\mathrm{J}(\mathrm{x}, \mathrm{y})]$ and phase space (Fig. 5.26). The transverse beam distribution of the clearing case had a smaller circular distribution compared to the larger and more irregular shapes of the other two 

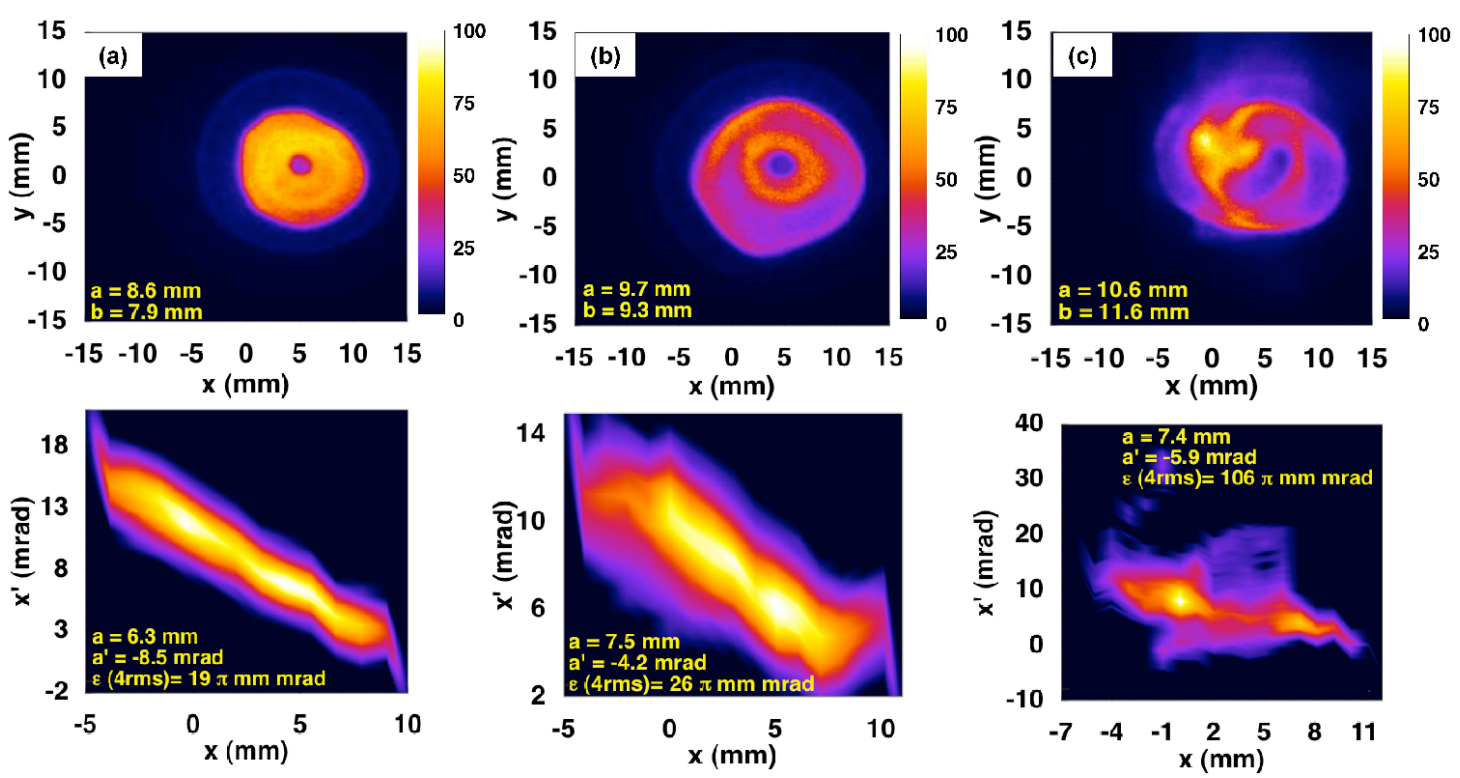

Figure 5.26. Top row: measured transverse beam distribution $56 \mathrm{~cm}$ downstream of the exit of four solenoids $(\mathrm{z}=306 \mathrm{~cm})$; bottom row: measured transverse phase space $40 \mathrm{~cm}$ downstream of the exit of four solenoids $(\mathrm{z}=290 \mathrm{~cm}$; note scale differences) for: (a) clearing case; (b) grounded case; (c) trapping case.

cases. Grounding the electrodes matched the unnormalized emittance measured in Fig. 5.21(b) without any electron cloud diagnostics. However, it was almost 40\% greater than the clearing case, where electrons were collected in the gaps between solenoids. Trapping electrons inside the solenoids increased the unnormalized emittance of the clearing case by more than a factor of five. Despite the reduced emittance from electron clearing, the beam quality was not as desired with a large $(>5 \mathrm{~mm})$ centroid offset, $>25 \%$ beam halo and a hollow non-axisymmetric distribution. The likely causes of this degradation in the beam quality is discussed in Sections 5.3 .3 \& 5.6 and also in Refs. [11, 35-37].

Further evidence of efficient clearing of electrons was shown in separate time dependent phase space measurements of the beam (Fig. 5.27). The sheared transverse phase space of the clearing case had a fairly uniform distribution compared to the larger and more distorted shape of the case where the electrodes were grounded. 
Again the case where the electrodes were grounded had a larger emittance compared to collecting electrons in the gaps between solenoids (clearing), which matched the emittance of the beam extracted from the diode in Fig. 5.21(a) (14 $\pi \mathrm{mm} \mathrm{mrad})$. The emittance from these particular optical measurements is $>20 \%$ larger than the time dependent phase space measurements, but the trend of electron clearing is the same. Clearing electrons also removed the time dependence in the beam envelope and parameters agreed well with values calculated by solving the envelope equation in Fig. 5.20(b). The variation in the beam envelope of the clearing case on the left was within the resolution of the measurement, which is less than a millimeter and a milliradian. Similar to Fig. 5.21(c) the spikes in Fig. 5.27 just after $3 \mu$ s and just before $13 \mu$ s are due to image currents from the head and tail of the beam pulse. The spike after $10 \mu$ s corresponds to amplified electrical noise from the crowbar spark gap firing to terminate the Marx voltage pulse at $10 \mu \mathrm{s}$ (Fig. 5.2).

The time dependence observed in the beam envelope without clearing electron clouds was likely due to partial neutralization of the beam space charge. If the electron density was high enough at any location in the lattice that portion of the beam envelope would have focused more easily as was also seen in the two-solenoid experiment (Section 5.3.1) [12]. The results of these time-dependent measurements (Fig. 5.27) and the optical measurements (Fig. 5.26) demonstrate the impact of clearing electrons. Combining these results with the measured ratio of $\lambda_{\mathrm{e}} / \lambda_{\mathrm{b}} \sim 1-6 \%$ when clearing electrons confirms partial neutralization 1-6\% can cause the observed increase in emittance. This also makes the case stronger that the aperture is the leading source of electrons and gas and the electrodes surrounding it are ineffective alone as electron suppressors in a solenoid lattice. 

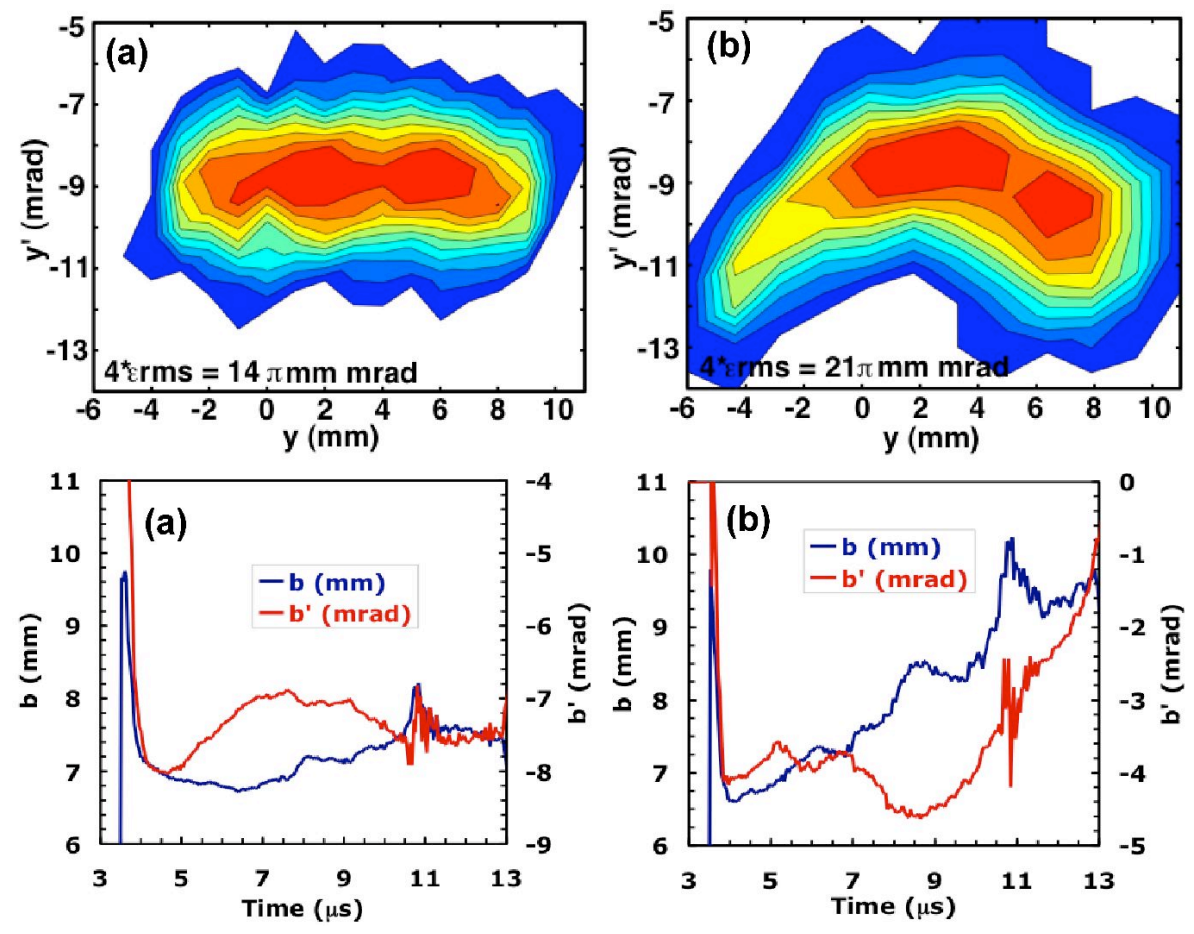

Figure 5.27. Top row: measured sheared phase space distribution; bottom row: measured envelope as a function of time for: (a) clearing case; (b) grounded case; all 40 $\mathrm{cm}$ downstream of the exit of four solenoids $(\mathrm{z}=290 \mathrm{~cm})$. 

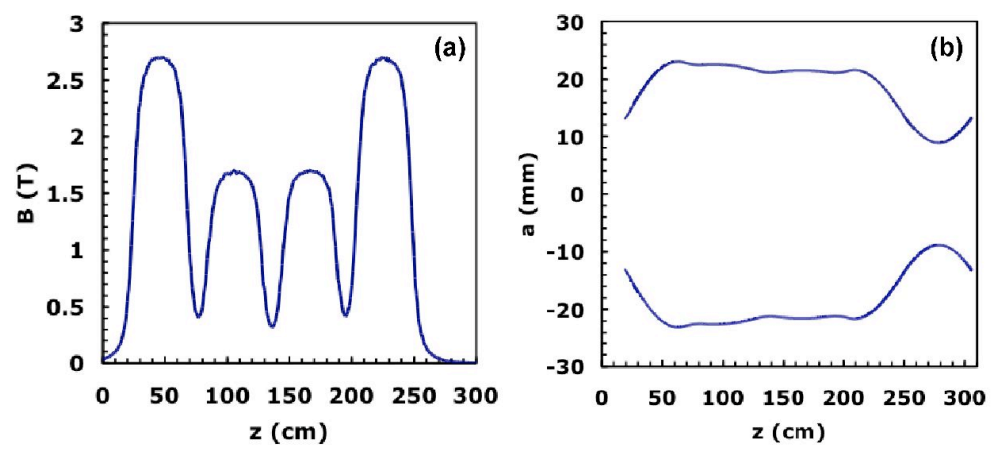

Figure 5.28. (a) Axial magnetic field profile based on measurements; (b) calculated envelope of 45-mA beam.

\subsubsection{Unapertured beam measurements with electron cloud diagnostics}

The evidence that the aperture was a leading source of electrons and gas was complemented with measurements without the aperture. Removing the aperture increases the current from 26 to $45 \mathrm{~mA}$, so a slightly different focusing strength [Fig. $5.28(\mathrm{a})]$ had to be used in the lattice to compensate for the higher beam current and in order to yield an envelope that did not scrape [Fig. 5.28(b)]. To provide a beam that was not too large to measure at the focal plane, a radius of $\leq 25 \mathrm{~mm}$ in the solenoid lattice was chosen. The magnetic field strength of solenoids 1 and 4 were increased to $2.7 \mathrm{~T}$ and solenoids 2 and 3 were increased by the square root of the current ratio $(\sim 30 \%)$.

The measurements with the unapertured 45-mA beam show there was an insignificant difference between the cases when the diagnostics were grounded versus when they were biased to clear electrons; and negligible charge was collected on the electron cloud diagnostics for the 45-mA beam when compared with the clearing case for apertured 26-mA beam (Table 5.2 and Fig. 5.29). The highlighted cases in Table 5.2 show the most significant differences in the charge collected on individual electrodes. 
Table 5.2. Comparison of the charge collected on the electron cloud diagnostics for the $45-\mathrm{mA}$ beam and the apertured 26-mA beam for the clearing case.

\begin{tabular}{|c|c|c|c|}
\hline & & 26-mA beam & 45-mA beam \\
\hline Diagnostic & Voltage (kV) & Charge (nC) & Charge (nC) \\
\hline electrode 1 & -1 & 1 & -1 \\
\hline electrode 2 & +1 & -41 & -1 \\
\hline electrode 3 & -1 & -2 & 0 \\
\hline electrode 4 & +1 & -11 & -1 \\
\hline electrode 5 & -1 & 9 & 0 \\
\hline electrode 6 & +1 & -2 & -2 \\
\hline electrode 7 & -1 & -1 & -1 \\
\hline electrode 8 & +1 & -19 & 0 \\
\hline & & & 6 \\
\hline Total Charge (nC) & & 86 & \\
\hline
\end{tabular}
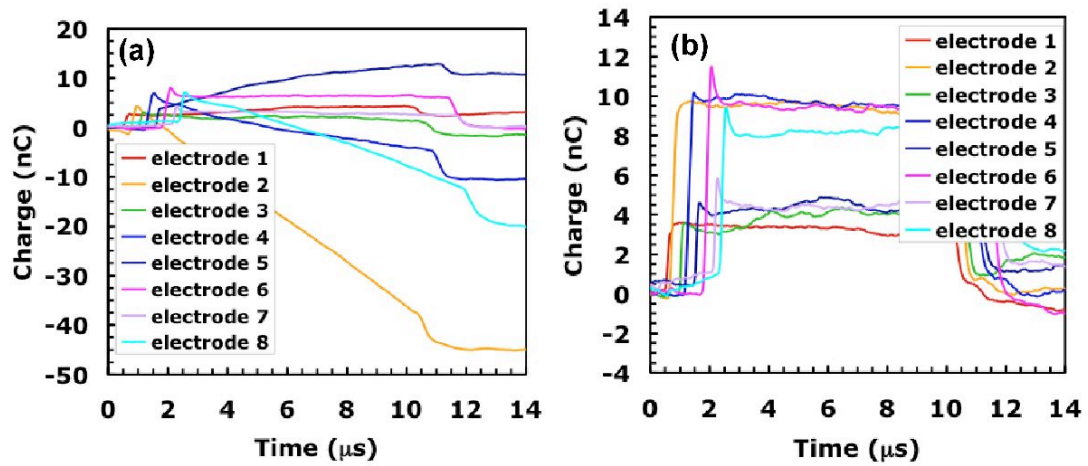

Figure 5.29. Comparison of the charge collected on the electron cloud diagnostics for the: (a) apertured 26-mA beam and; (b) 45-mA beam; both for the clearing case (note scale differences).

Those electrodes with $2 \mathrm{nC}$ or less of collected charge were well within the electrical noise (error bars) of the measurements. This confirmed that clearing electrodes are not necessary for a short accelerator like this if there is no other source of electrons and gas besides the aperture.

Examining the charge collected on the electrodes due to capacitive effects for the 45-mA beam demonstrated the difference in electrode length [Fig. 5.29(b)]. The shorter electrodes had $4 \mathrm{nC}$ of induced charge, while the longer electrodes showed about $10 \mathrm{nC}$. The accumulated charge (difference between end and beginning of beam pulse) for the unapertured case showed a $>10 \mathrm{x}$ reduction compared to the apertured 

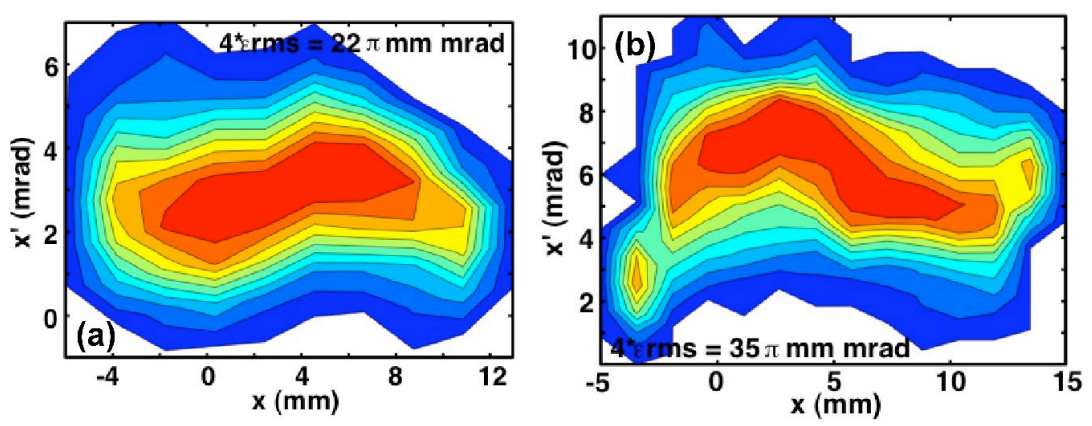

Figure 5.30. Sheared phase space distribution of the unapertured 45-mA beam integrated over a $500 \mathrm{~ns}$ gate in the middle of the $10 \mu \mathrm{s}$ beam pulse: (a) $40 \mathrm{~cm}$ downstream of the exit of two solenoids $(\mathrm{z}=171 \mathrm{~cm})$; (b) $40 \mathrm{~cm}$ downstream of the exit of four solenoids $(\mathrm{z}=290 \mathrm{~cm}$; note scale differences).

beam, in spite of almost twice the beam current and a larger beam envelope (Table $5.2)$.

The measurements without the aperture in this four-solenoid lattice showed a $50 \%$ increase in emittance from that measured at the gun and after two solenoids (Fig. 5.30). As stated above electron cloud effects appear to be small for this unapertured beam case and time dependence in the beam envelope is also small. The distortions in the phase space distribution (Fig. 5.30) add to the emittance and are most likely due to the centroid motion of the beam throughout the focusing lattice $[12,37]$.

\subsection{Beam induced gas and electrons}

The pair of polished stainless steel parallel plates (Fig. 5.31) described in Section 3.4.2 were used to make measurements of beam induced gas and electrons to benchmark electron cloud models and codes. Two configurations were used: first both plates were biased negatively to suppress electrons, and second the plates were biased as a dipole with one plate biased positively to sweep electrons and the other grounded to collect some of the ionized gas. The parallel plates were placed $\sim 1 \mathrm{~cm}$ 


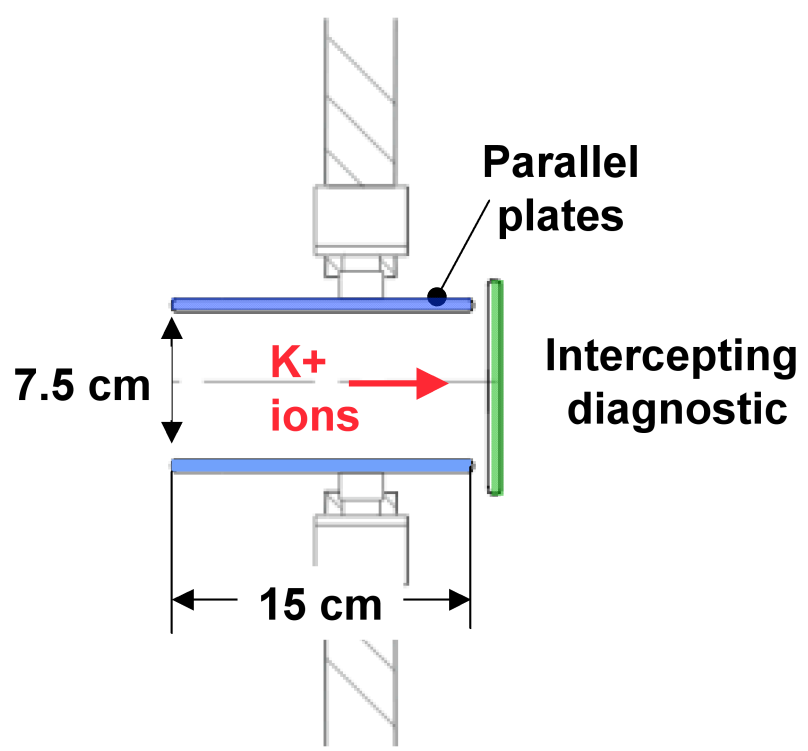

Figure 5.31. Sketch of the experimental setup using the parallel plates and intercepting diagnostic to measure beam induced gas desorption, ionization, and electron emission.

upstream of the intercepting diagnostic and biased from 0 to $10 \mathrm{kV}$ in $1 \mathrm{kV}$ intervals.

As described in Section 3.4.2, a stainless steel plate and a copper plate, were used in consecutive data sets to intercept the beam at $\mathrm{z}=290 \mathrm{~cm}$. The purpose of using the two materials was to test whether the amount of gas desorbed and electrons emitted was strongly dependent on material composition.

As mentioned earlier, these experiments were conducted with a $10 \mu \mathrm{s}$, singly charged $\mathrm{K}^{+}$ion bunch at an ion energy of $0.3 \mathrm{MeV}$ and current of $26 \mathrm{~mA}$ providing 1.6 $\times 10^{12}$ ions/pulse. The repetition rate of the ion pulse was flexible but was maintained at $0.05 \mathrm{~Hz}$. Target heating of the plates was negligible $(\ll 0.1 \mathrm{eV})$ because the range of the ions at this energy $(300 \mathrm{keV})$ is less than $1 \mu \mathrm{m}$. Our typical vacuum pressure, $4-5 \times 10^{-8}$ torr, was used in these experiments because we wanted to quantify beam 


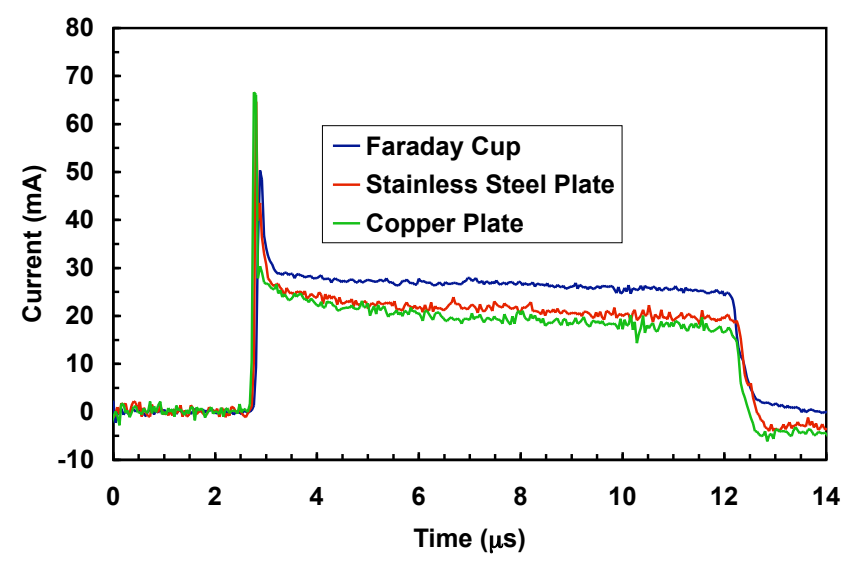

Figure 5.32. Beam current signals collected by the Faraday cup (blue), stainless steel plate (red), and copper plate (green) with both parallel plates biased negatively.

induced gas desorption, ionization, and electron emission for our normal operating conditions. No significant change in vacuum pressure was noted for these experiments.

The intercepting plates (targets) were capacitively monitored at $0 \mathrm{~V}$ and the signal provided with both parallel plates biased negatively was slightly reduced from the measured beam current due to non-beam ion and electron species in the vicinity of the diagnostic (Fig. 5.32). Once the head of the beam had passed, after $3 \mu \mathrm{s}$, the beam current and envelope stabilized, and the number of emitted gas molecules and electrons should rise linearly over the relevant time frame $(10 \mu \mathrm{s})$ along with the collected current. This assumes that the size of the beam hitting the target is large enough that the gas expansion is negligible in the transverse direction.

\subsubsection{Ionized gas measurement}

Positive ions, from the ionization of gas desorbed off the intercepting plate, were measured with both parallel plates biased negatively. Although this is a useful measurement, it would be more informative if the parallel plates were biased as a dipole with one plate biased negatively to collect all of the gas ions and the other grounded 
to collect some of the electrons. With both plates biased negatively there is no field between the plates and it is possible that gas expanding from the center of the target could escape. $\mathrm{H}_{2}$ gas was the likely candidate of gas to be desorbed from stainless steel at a rate of $\sim 3000 \mathrm{H}_{2}$ molecules $/ \mathrm{K}^{+}$ion based on measurements [58]. It is inferred from these measurements and simulations that the subsequently ionized gas is a mixture of $\mathrm{H}_{2}{ }^{+}$and $\mathrm{H}^{+}$.

The calculated time for a positive current to appear on the parallel plates due to ionized $\mathrm{H}_{2}$ was about $1 \mu \mathrm{s}$. On the NDCX there was a positive capacitive image current when the beam head passed through the diagnostic at $2.5 \mu$ s followed by a rising positive current less than $1 \mu$ s later [Fig. 5.33(a)]. The gas initially desorbed and ionized $\left(\mathrm{I}_{\mathrm{H}}\right)$ was measured immediately after the beam head at $3.2 \mu \mathrm{s} . \mathrm{I}_{\mathrm{H}}$ measured with the stainless steel target was $20 \%$ of the $\mathrm{K}^{+}$ion current $\left(\mathrm{I}_{\mathrm{b}}\right)$ with the plates biased to $-1 \mathrm{kV}$ and increased linearly to $>30 \%$ when the plates were biased to -10 $\mathrm{kV}$ [Fig. 5.33(b)]. These values were slightly higher for copper, $\mathrm{I}_{\mathrm{H}} / \mathrm{I}_{\mathrm{b}}=25 \%$ at $-1 \mathrm{kV}$ and increased linearly to $39 \%$ at $-10 \mathrm{kV}$. This bias dependence suggests a saturation of the measured gas initially desorbed and ionized may require a bias above $10 \mathrm{kV}$. Assuming a similar amount of gas was desorbed per $\mathrm{K}^{+}$ion as in Ref. [58] then a very low fraction $\left(\sim 10^{-4}\right)$ of the gas molecules are ionized and collected on the parallel plates based on a measured and calculated cross sections. The relevant cross section is the sum of the ionization cross section and the charge-exchange cross section, which is $\sim 1 \times 10^{-19} \mathrm{~m}^{2}$ according to measurements and Slater, and Thomas-Fermi models [127].

The initially steep slope of the ionized gas current at $3.2 \mu$ s decreased as function of time [Fig. 5.33(a)]. This was due to expansion of the gas transversely. The beam was only $1.5 \mathrm{~cm}$ wide, which is small compared to the distance between the plates $(7.5 \mathrm{~cm})$, so the gas did not continue to be ionized at the same rate as it was initially. 

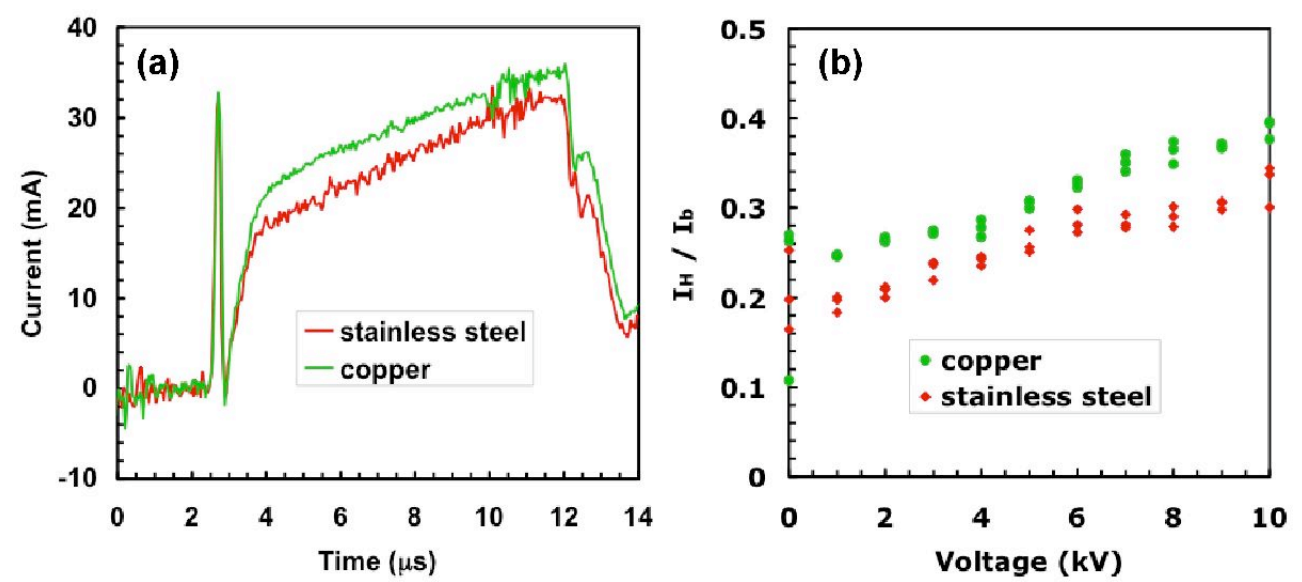

Figure 5.33. (a)Ionized gas current collected on the parallel plates biased at $-10 \mathrm{kV}$ when intercepting the beam with a stainless steel plate (red) and a copper plate (green); (b) ratio of the ionized gas current $\left(\mathrm{I}_{H}\right)$ collected on the negatively biased parallel plates to the beam current $\left(\mathrm{I}_{b}\right)$ versus bias potential over a 200 ns gate at 3.2 $\mu \mathrm{s}$.

Nonetheless there was still a positive slope and the total ion current (or charge) from the gas desorbed off of the stainless steel plate was $87 \%$ of the total $\mathrm{K}^{+}$ion current and for the copper plate it was almost equal to the total $\mathrm{K}^{+}$ion current.

The ionized gas current measured on the parallel plates was used to quantify the ionized gas line charge density $\left(\lambda_{\mathrm{H}}=\mathrm{I}_{\mathrm{H}} / \mathrm{v}_{\mathrm{H}}\right)$ immediately after the beam head and at the end of the beam pulse. Assuming the gas ions are $\mathrm{H}^{+}$and they have kinetic energy (10 keV) provided by the potential difference from the parallel plates to grounded intercepting electrode, then $\mathrm{H}^{+}$ions have a $\mathrm{v}_{\mathrm{H}}=1.39 \times 10^{6} \mathrm{~m} / \mathrm{s}$. Initially, this yields $\lambda_{\mathrm{H}}=6 \mathrm{nC} / \mathrm{m}$ when intercepting the beam with the stainless steel plate and $7.2 \mathrm{nC} / \mathrm{m}$ when intercepting the beam with the copper plate (Table 5.3). These measurements directly after the beam head have an uncertainty of $<5 \%$. Including the rest of the beam pulse, $\lambda_{\mathrm{H}}$ increases to $16.2 \mathrm{nC} / \mathrm{m}$ for the stainless steel case and $18.3 \mathrm{nC} / \mathrm{m}$ for the copper case with an uncertainty of about $1 \%$. Immediately after the beam head and at the end of the beam pulse there are about $15 \%$ more gas ions collected when 
Table 5.3. Calculated ionized gas densities immediately after the beam head (3.2 $3.4 \mu \mathrm{s})$ and at the end of the beam pulse when intercepting the beam with stainless steel and copper plates.

\begin{tabular}{|c|c|c|c|c|}
\hline Time $(\boldsymbol{\mu s})$ & \multicolumn{2}{|c|}{$\mathbf{3 . 2 - 3 . 4}$} & \multicolumn{2}{c|}{$\mathbf{2 . 5 - 1 2 . 5}$} \\
\hline Material & SS & $\mathbf{C u}$ & SS & $\mathbf{C u}$ \\
\hline $\boldsymbol{\lambda}_{\mathrm{H}}(\mathbf{n C} / \mathbf{m})$ & 6.05 & 7.19 & 16.24 & 18.29 \\
\hline $\mathbf{n}_{\mathbf{H}}\left(\mathbf{c m}^{-3}\right)$ & $2.14 \mathrm{E}+08$ & $2.54 \mathrm{E}+08$ & $5.75 \mathrm{E}+08$ & $6.47 \mathrm{E}+08$ \\
\hline
\end{tabular}

intercepting the beam with copper versus stainless steel. This demonstrates a slight material dependence for two plates with identical surface preparation. Although, a more detailed study of different surface preparations may yield different results, the objective was to study different materials with our standard vacuum surface preparation.

The ionized gas density $\left(\mathrm{n}_{\mathrm{H}}\right)$ is inferred using the beam line charge density $\left(\lambda_{\mathrm{b}}=\right.$ $21 \mathrm{nC} / \mathrm{m})$ and the values calculated above assuming $\mathrm{n}_{\mathrm{H}} / \mathrm{n}_{\mathrm{b}} \propto \lambda_{\mathrm{H}} / \lambda_{\mathrm{b}}$, where $\mathrm{n}_{\mathrm{b}}(7.4 \times$ $10^{8} \mathrm{~cm}^{-3}$ ) is the beam density at the intercepting diagnostic. The resulting densities are shown in Table 5.3. $\mathrm{n}_{\mathrm{H}}$ early in the beam pulse is only about $30 \%$ of the beam density but increases to $77 \%$ of the beam density for the stainless steel case and $87 \%$ for the copper case. This time dependence is expected from the slope in [Fig. 5.33(a)] and the final densities are close to the beam density which is in good agreement with what is predicted for beam induced gas desorption by particle in the cell simulations using the Warp code $[59,94]$.

\subsubsection{Electron emission measurement}

Biasing the parallel plates in a dipole configuration collected all of the secondary electrons due to beam induced emission and ionization of desorbed gas on the positively biased plate and a fraction of the desorbed and further ionized hydrogen on the grounded plate. This experiment provided the number of electrons released per 
ion for normal incidence $\left(\gamma_{\mathrm{se}}\right)$. Assuming the cross sections for beam or gas ion recombination and second ionization are small, the accumulated electron current from 3.4-4 $\mu \mathrm{s}$ had a slope in time that was proportional to the product of the desorbed gas density $\left(\mathrm{n}_{\mathrm{H}}\right)$ and the ionization cross section $\left(\sigma_{\mathrm{iz}}\right)$.

A positive capacitive image current appeared when the beam passed through the diagnostic at $2.5 \mu$ s followed by an almost instantaneous negative capacitive spike from secondary electrons released from the intercepting plate by the beam head [Fig. 5.34(a)]. Once the head of the beam had passed the beam current and envelope stabilized providing a consistent determination of the number of secondary electrons. The secondary emission coefficient $\left(\gamma_{\mathrm{se}}\right)$, the ratio of the secondary electron current $\left(\mathrm{I}_{\mathrm{e}}\right)$ collected on the positively biased parallel plate to the beam current $\left(\mathrm{I}_{\mathrm{b}}\right)$, was measured immediately after the beam head at $3.2 \mu$ s over a 200 ns gate [Fig. 5.34(b)]. The stainless steel target produced a $\gamma_{\mathrm{se}}=4.42 \pm 0.05$ with the parallel plate biased to $+1 \mathrm{kV}$ and increased to $10.87 \pm 0.11$ when the plate was biased to $+10 \mathrm{kV}$. The value was about the same for copper at $+1 \mathrm{kV}$, but increased at a slower rate to $9.18 \pm 0.02$ at $+10 \mathrm{kV}$. This bias dependence suggests a saturation of the measured secondary electron current may require a bias above $10 \mathrm{kV}$ as was seen with the ionized gas measurements [Fig. 5.33(b)].

A large difference in the dynamics of the electron emission was seen when intercepting the beam with stainless steel versus copper. When the stainless steel plate intercepted the beam there was an initial negative slope in the electron current at $3.2 \mu$ s that decreased (became less negative) as function of time until $7 \mu$ s when a small positive spike was observed [Fig. 5.34(a)]. The positive spike is believed to be a sheath of hydrogen ions that was forced to the positive plate due to saturation of the electron sheath. At about $7.5 \mu$ s the electron current recovered and saturated at $\sim$ $390 \mathrm{~mA}$. These trends were seen at lower bias voltages with later saturation times and 

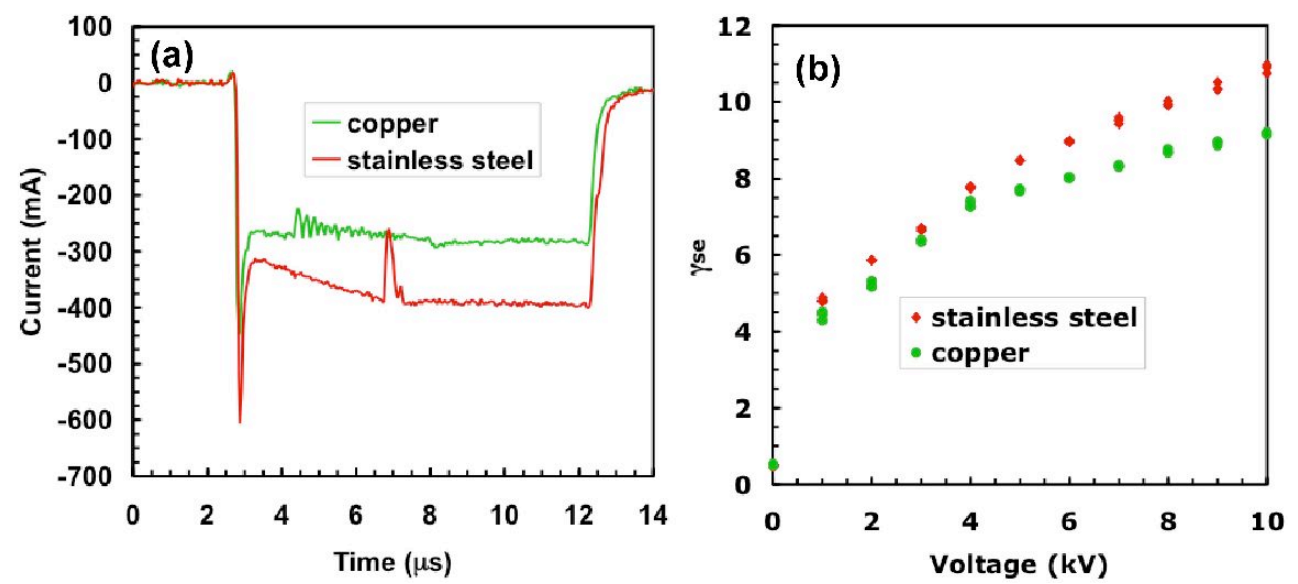

Figure 5.34. (a) Secondary electron current collected on the parallel plate biased at $+10 \mathrm{kV}$ when the beam is intercepted with a stainless steel plate (red); and a copper plate (green); (b) Secondary emission coefficient versus bias potential over a $200 \mathrm{~ns}$ gate at $3.2 \mu \mathrm{s}$.

lower saturation currents. The decreasing negative slope in the electron current was just more evidence of the transverse expansion of the gas leading to less ionization and a reduced slope in electron current. This implies $n_{H}$ and $n_{e}$ are a function of space and time.

Intercepting the beam with the copper plate displayed a different phenomena; a flat electron current at $3.2 \mu$ s was observed followed by a high frequency oscillation at $4.5 \mu \mathrm{s}$. This oscillation is believed to be alternating sheaths of hydrogen ions and electrons. These characteristics were also seen at lower bias voltages.

The secondary electron current measured on the positive parallel plate was used to quantify the electron line charge density $\left(\lambda_{\mathrm{e}}=\mathrm{I}_{\mathrm{e}} / \mathrm{v}_{\mathrm{e}}\right)$ immediately after the beam head and at the end of the beam pulse. Assuming the electrons have kinetic energy (10 keV) provided by the potential difference from the positively biased parallel plate to grounded intercepting electrode, then they have a $\mathrm{v}_{\mathrm{e}}=5.94 \times 10^{7} \mathrm{~m} / \mathrm{s}$. Initially, this yields $\lambda_{\mathrm{e}}=4.69 \mathrm{nC} / \mathrm{m}$ when intercepting the beam with the stainless steel plate and $3.96 \mathrm{nC} / \mathrm{m}$ when intercepting the beam with the copper plate (Table 5.4). These 
Table 5.4. Calculated secondary emission coefficient and electron densities immediately after the beam head $(3.2-3.4 \mu \mathrm{s})$ and at the end of the beam pulse when intercepting the beam with stainless steel and copper plates.

\begin{tabular}{|c|c|c|c|c|}
\hline Time $(\boldsymbol{\mu s})$ & \multicolumn{2}{|c|}{$\mathbf{3 . 2 - 3 . 4}$} & \multicolumn{2}{c|}{$\mathbf{2 . 5 - 1 2 . 5}$} \\
\hline Material & SS & $\mathbf{C u}$ & $\mathbf{S S}$ & $\mathbf{C u}$ \\
\hline$\gamma_{\mathbf{s e}}$ & 10.87 & 9.18 & - & - \\
\hline$\lambda_{\mathbf{e}}(\mathbf{n C} / \mathbf{m})$ & 4.69 & 3.96 & 5.83 & 4.29 \\
\hline $\mathbf{n}_{\mathbf{e}}\left(\mathbf{c m}^{-3}\right)$ & $1.66 \mathrm{E}+08$ & $1.40 \mathrm{E}+08$ & $2.06 \mathrm{E}+08$ & $1.52 \mathrm{E}+08$ \\
\hline
\end{tabular}

measurements directly after the beam head have an uncertainty of about $1 \%$. Including the rest of the beam pulse $\lambda_{\mathrm{e}}$ increases to $5.83 \mathrm{nC} / \mathrm{m}$ for the stainless steel case and $4.29 \mathrm{nC} / \mathrm{m}$ for the copper case with an uncertainty of $<1 \%$. Immediately after the beam head and at the end of the beam pulse there are $\geq 15 \%$ more electrons collected when intercepting the beam with stainless steel versus copper; again showing a slight material dependence.

Similar to $n_{H}$, the electron density $\left(n_{e}\right)$ is inferred using $\lambda_{b}$ and the values calculated above assuming $\mathrm{n}_{\mathrm{e}} / \mathrm{n}_{\mathrm{b}} \propto \lambda_{\mathrm{e}} / \lambda_{\mathrm{b}}$ (Table 5.4). Early in the beam pulse $\mathrm{n}_{e}$ is only about $22 \%$ of the beam density but increases to $28 \%$ of the beam density for the stainless steel target. Intercepting the beam with the copper plate $\mathrm{n}_{e}$ is only about $20 \%$ of the beam density throughout the whole beam pulse. Unlike the measured ionized hydrogen gas there is not much time dependence in the measured electron densities. However, they are on the same order of magnitude as the beam density and that is in good agreement with what is predicted by Warp simulations of beam induced gas desorption [59, 94].

These measurements demonstrate a difference in the dynamics of the ionized gas and electron currents measured and a slight material dependence. Measurements from a residual gas analyzer and $\mathrm{K}^{+}$ion beam normally incident upon stainless steel indicate that the dominant gas species desorbed is hydrogen [58]. The next most abundant species is nearly an order of magnitude less, had an atomic mass of 28 which 
could be $\mathrm{N}_{2}$ or CO gas. Assuming hydrogen gas is the dominant species desorbed from the copper target, it appears from the ionized gas measurements that $15 \%$ more hydrogen is desorbed from the copper target and then ionized by the beam. However, the number of electrons emitted is $\geq 15 \%$ higher when intercepting the beam with stainless steel versus copper. These results make it difficult to conclude whether one material is more suitable than the other and further investigation may be necessary.

\subsection{Centroid Motion}

In the previous sections of this chapter (Sections $5.3 \& 5.4$ ) it was shown that the beam has centroid offsets of several $\mathrm{mm}$ and mrad in addition to distortions in the current density and phase space distributions. Precise alignment of the axial magnetic field in a solenoid lattice is critical to the beam dynamics. Slight misalignment of the solenoids in a focusing lattice causes the beam centroid to carry out a corkscrew orbit and this motion can grow along a focusing lattice if each additional solenoid is misaligned $[35,36]$. This excitation can also lead to emittance growth and halo formation [37].

Experiments were conducted and an analytic model was also developed to understand these effects and possibly mitigate them. Steering dipoles have been used in past solenoid transport experiments with electron beams and were also used here to correct the centroid motion $[116,117]$.

\subsubsection{Measured centroid offsets}

A series of transverse phase space and transverse beam distribution $[\mathrm{J}(\mathrm{x}, \mathrm{y})]$ measurements were made with different lattice tunes to quantify the centroid dependence 

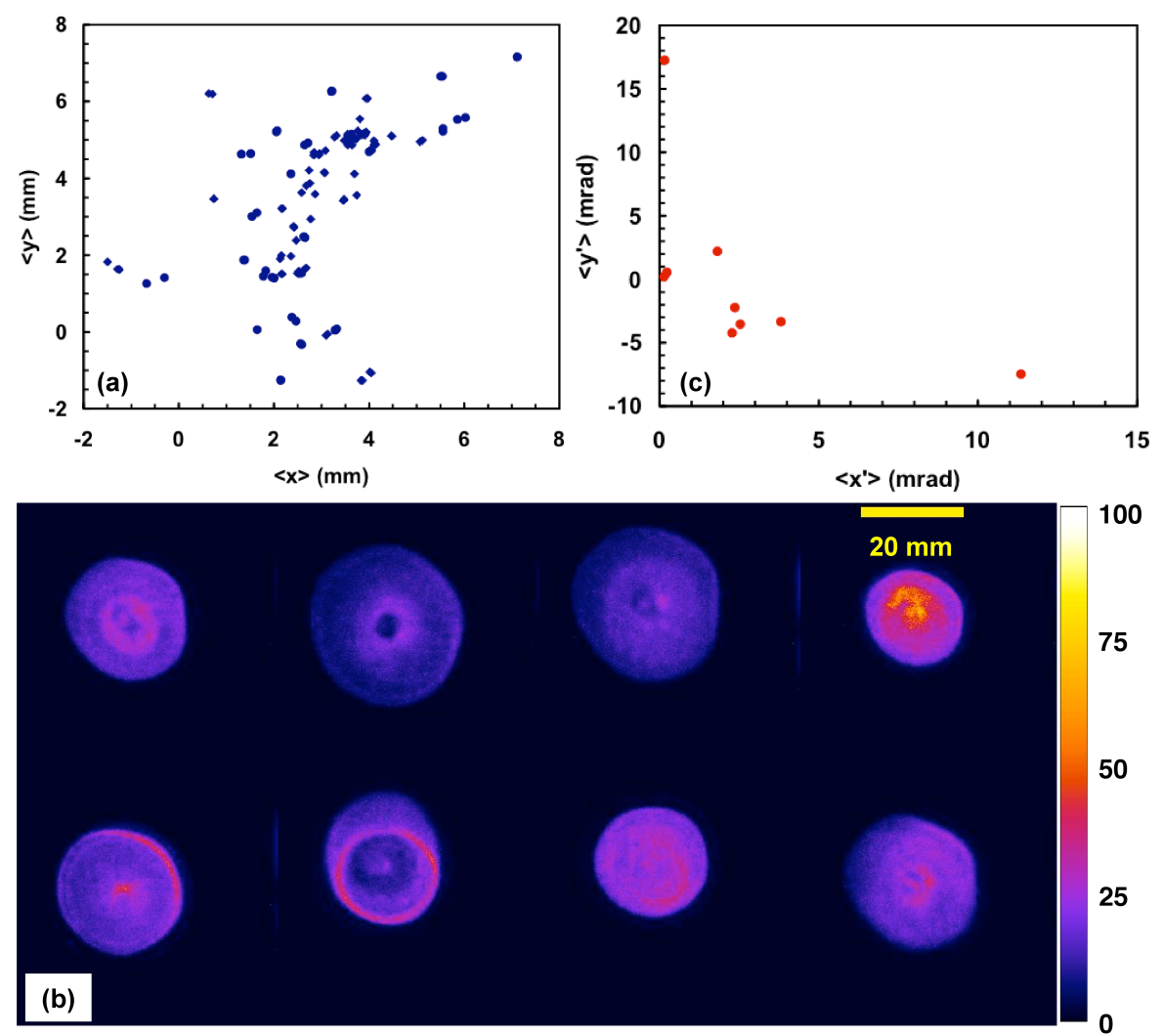

Figure 5.35. (a) Measured position offsets from $\mathrm{J}(\mathrm{x}, \mathrm{y})$ measurements for 48 different lattice tunes; (b) measured $\mathrm{J}(\mathrm{x}, \mathrm{y})$ for a subset of eight of these tunes and; (c) measured angular offsets from 4-D phase space measurements. All measured at the exit of the four solenoid lattice.

on individually unique lattice tunes. $\mathrm{J}(\mathrm{x}, \mathrm{y})$ was measured for 48 different tunes, 22 of these tune were individually unique (each value for each element in the lattice was different for each case). The position offsets $(\langle\mathrm{x}\rangle,\langle\mathrm{y}\rangle)$ were measured from all of these distributions and are displayed in Fig. 5.35(a). The observed centroid offsets are all confined to one region of configuration space demonstrating a constraint (or confinement) of the beam centroid by the misaligned focusing elements. The mean of the centroid values from the data in Fig. 5.35(a) were $\langle\mathrm{x}\rangle=2.96 \pm 1.46 \mathrm{~mm}$ and $\langle\mathrm{y}\rangle=3.42 \pm 2.13 \mathrm{~mm}$.

$\mathrm{J}(\mathrm{x}, \mathrm{y})$ from a subset of eight of the 22 unique tunes is shown in Fig. 5.35(b). 
It is worth noting the variation in intensity of the distributions and the movement of the beam centroid. In addition to the $J(x, y)$ measurements, 4-D phase space measurements were made for a subset of 8 tunes from 48 mentioned above. Five of the tunes were individually unique and each of the eight had different angular offsets $\left(\left\langle\mathrm{x}^{\prime}\right\rangle,\left\langle\mathrm{y}^{\prime}\right\rangle\right)$ [Fig. 5.35(c)]. In principle, these measurements provide more than an adequate amount of data to solve for the individual displacements of the solenoids relative to the ideal beam axis and the necessary corrections needed to suppress the betatron motion of the beam centroid. However, the uncertainty in the data may limit the reliability and convergence of an inversion to calculate the magnet offsets. Determining this information from measurements of $\mathrm{J}(\mathrm{x}, \mathrm{y})$ greatly reduces data acquisition time and analysis of the data, simplifying the problem for future applications.

\subsubsection{Dipole magnets}

Dipole magnets were fabricated for centroid corrections on the NDCX. A pair of $\mathrm{x}$ and $\mathrm{y}$ dipoles were designed for each of the three gaps between the four solenoids. The dipoles were wound from 1-mm diameter copper wire. Each dipole consisted of two half-shells with 39 turns each; a CAD model of a single half-shell coil is shown in Fig. 5.36(a). The y-field dipole (for x-plane bends) had a coil radius and axial length of $51.3 \mathrm{~mm}$ and $52.1 \mathrm{~mm}$. The x-field dipole (for y-plane bends) had a slightly smaller radius $(47.5 \mathrm{~mm})$ and longer $(71.1 \mathrm{~mm})$ coil in order to nest the dipole pair together. Photographs of an assembled dipole pair is shown in Fig. 5.36(b). The finished assembly fits in the $9 \mathrm{~cm}$ gaps between the solenoids and around the 9-cm diameter beam pipe [Fig. 5.36(c)]. A minimum of two steering dipole pairs are required to correct the beam centroid at the exit of the transport lattice, however three were fabricated to minimize centroid motion throughout the lattice. 


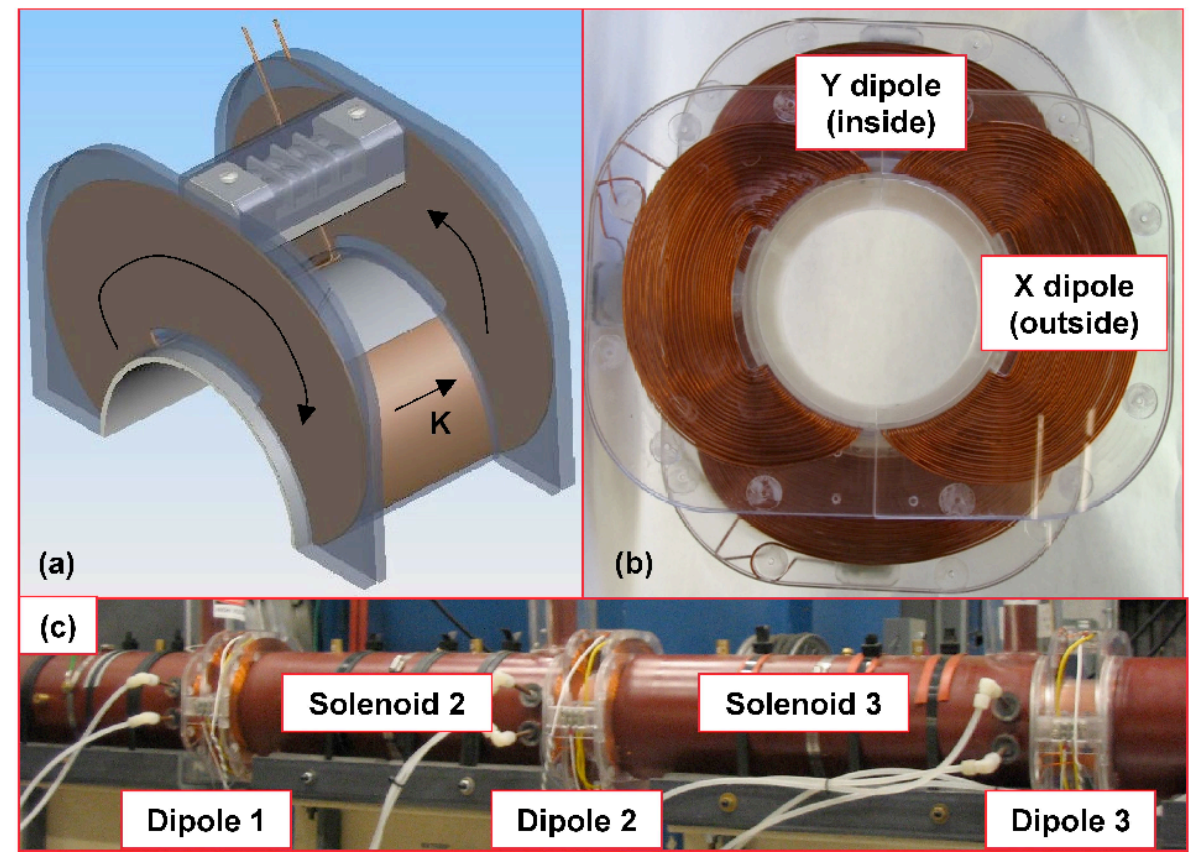

Figure 5.36. (a) CAD model of a dipole half-shell with the direction of the sheet current (K) shown; (b) a photo of assembled dipole magnet pair with $\mathrm{x}$ and $\mathrm{y}$ dipoles labeled and; (c) a photo of assembled dipole magnet pairs installed on NDCX. 
Each dipole was driven independently by a 2-ms pulse provided by a pulse forming network. The peak voltage and current amplitudes were $~ 200$ Volts and 200 Amps, producing magnetic fields $>500 \mathrm{G}$. This has been confirmed with an approximate, 2-D analytical model which yields a field function of

$$
B_{x, y}=\frac{3 \sqrt{3}}{2 \pi^{2}} \frac{\mu_{o} N I}{r},
$$

where $N$ is the number of turns, $I$ is the current applied, and $r$ is the radius of the coil. The field strength generated is approximately constant over the $10-\mu$ s beam pulse and is capable of applying $>10$ milliradian kicks to the $300 \mathrm{keV} \mathrm{K} \mathrm{K}^{+}$ion beam.

\subsubsection{Analytic method}

An analytic method was developed to examine the offsets of individual solenoids and quantify their individual and total contributions to the centroid motion [118]. In this model a fit to the measured field for each solenoid is given a position and angular displacement with respect to the ideal beam centerline. The resulting field is resolved into an ideal component plus dipole terms related to the misalignment parameters. Equations of motion are then derived for the transverse centroid evolution. These equations of motion are expressed in the rotating Larmor frame where they are most simply expressed. An analogy to dispersion functions is exploited to derive a linear, small-amplitude expansion of the centroid orbit in terms of 3 components:

1. The centroid motion due to initial condition errors at the emitter $\left(\left\langle\mathrm{x}_{\mathrm{o}}\right\rangle,\left\langle\mathrm{x}_{\mathrm{o}}^{\prime}\right\rangle\right)$ evolving through an ideally aligned lattice.

2. The centroid motion due to mechanical misalignments of each solenoid. This is expressed in terms of position and angular displacements for each solenoid. 

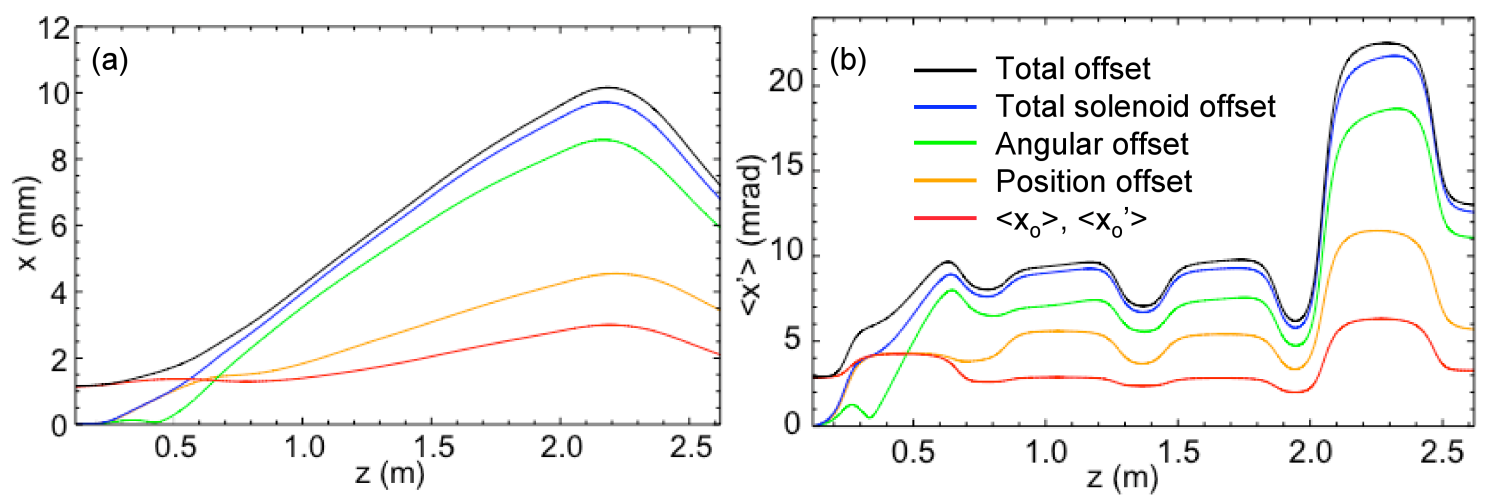

Figure 5.37. Statistical contributions of individual misalignments to the centroid offsets $(\mathrm{a})\langle\mathrm{x}\rangle ;(\mathrm{b})\left\langle\mathrm{x}^{\prime}\right\rangle$ for a lattice tune of 2.6, 1.0, 0.8 and $2.05 \mathrm{~T}$.

3. The centroid motion due to dipole steering, expressed in terms of steering functions for each dipole only.

The rms $\mathrm{x}$-centroid $(\langle\mathrm{x}\rangle)$ due to an ensemble of 10,000 random error sets uniformly distributed up to cut-off values is shown in Fig. 5.37 for a lattice tune of 2.6, 1.0, 0.8 and $2.05 \mathrm{~T}$. The errors in the initial coordinate are cut-off at $2 \mathrm{~mm}$ and $5 \mathrm{mrad}$, and solenoid alignment errors are cutoff at $3 \mathrm{~mm}$ and $10 \mathrm{mrad}$ for position and angular displacements. Curves indicate rms contributions due to all errors added (black), solenoid position and angular offsets (blue), solenoid angular offsets only (green), solenoid position offsets only (orange), and initial offsets (red). The final centroid errors $(7.5 \mathrm{~mm}$ and $13 \mathrm{mrad})$ are in the range of typical values measured on NDCX (Fig. 5.35). It is worth noting, regardless of the tune, the solenoid angular offset contributes nearly twice as much to the final centroid offset compared to the position offset. A position offset of $2.5 \mathrm{~mm}$ is equivalent to an angular displacement of 10 mrad about the center of a 50-cm long solenoid. 
Table 5.5. Measured centroid correction at the exit of the four-solenoid lattice using the last two pairs of dipoles.

\begin{tabular}{|c|c|c|c|c|c|}
\hline & $\mathbf{z}(\mathbf{c m})$ & $\langle\mathbf{x}\rangle \mathbf{( m m})$ & $\left\langle x^{\prime}\right\rangle \mathbf{~ ( m r a d )}$ & $\langle\mathbf{y}\rangle \mathbf{( m m})$ & $\left\langle\mathbf{y}^{\prime}\right\rangle$ (mrad) \\
\hline Uncorrected & $\mathbf{2 6 2}$ & -5.81 & 2.24 & -2.77 & 3.37 \\
\hline Corrected & $\mathbf{2 6 2}$ & -0.15 & 0.51 & 0.00 & -0.31 \\
\hline
\end{tabular}

\subsubsection{Empirical correction}

A successful correction of the beam centroid was demonstrated empirically for the tune described above $(2.6,1.0,0.8$ and $2.05 \mathrm{~T})$ using the last two pairs of dipoles. After determining a close operating point of the last two dipole pairs a $4 \times 4$ Jacobian matrix was generated by measuring the four centroid offsets $\left(\langle\mathrm{x}\rangle,\langle\mathrm{y}\rangle,\left\langle\mathrm{x}^{\prime}\right\rangle\right.$, and $\left.\left\langle\mathrm{y}^{\prime}\right\rangle\right)$ for each of the four individual variations of the dipole current. Inverting the $4 \times 4$ Jacobian matrix, we were able to solve for the necessary operating currents for each of the four dipoles. The solution (Table 5.5) only used the last two pairs of dipoles and therefore only corrected the betatron motion of the beam centroid at the exit of the solenoid transport section.

This Jacobian-based procedure is quite laborious; it requires a 4-D phase space measurement for each of the four independent current variations. Since, the operating point of the dipoles is dependent upon the strength of the solenoids in the lattice, this solution susceptible to large errors when operating with a different tune. This empirical correction would have to be repeated every time the operating point of the lattice is changed. That is why the analytical method has been developed to determine the individual offsets of each lattice element. 


\subsection{Conclusions}

We successfully demonstrated matching and transport of a space-charge dominated ion beam in a two-solenoid lattice with little or no emittance growth. It is evident from the results presented in Section 5.3 that large fringe magnetic fields are responsible for electron confinement and contribute to the sheath formation and electron cloud effects observed, confusing measurements with intercepting diagnostics. Moving the beam intercepting diagnostics into a nearly magnetic field-free region provided the correct measurement of the beam dynamics and the emittance.

Precise placement of the emitter and alignment of the axial magnetic field in a solenoid lattice is critical to the beam dynamics. Evidence of this importance is seen in the measured and calculated beam distributions which are not uniform or axisymmetric, have a centroid offset of several millimeters and milliradians, a hollow center, and a substantial halo. Although these undesired effects have little impact on the emittance in the two solenoid experiment, they may grow in longer focusing lattices and contribute to emittance growth or beam loss.

Electron cloud studies were successfully conducted in a four-solenoid lattice using cylindrical electrodes that intercepted the expanding magnetic flux from the solenoids. Beam dynamics measurements and measurements from the electrodes confirmed that the current reducing aperture used in the experiments provided an $n_{e} \sim 0.01-0.06$ $\mathrm{n}_{\mathrm{b}}$, enough to partially neutralize the beam and cause the emittance to grow $\geq$ 40\%. Beam dynamics measurements proved that using the electrodes to clear these electrons was effective, prevented partial neutralization, and reduced the measured emittance so that it was conserved throughout the lattice.

The dynamics of beam-induced gas desorption, ionization, and electron emission

for normal incidence were characterized for a $10 \mu \mathrm{s}$, singly charged $\mathrm{K}^{+}$ion bunch 
at an ion energy of $0.3 \mathrm{MeV}$ and current of $26 \mathrm{~mA}$. This beam provided $1.6 \times 10^{12}$ ions/pulse at a repetition rate of $0.05 \mathrm{~Hz}$. These measurements showed the gas cloud continues to expand as a function of time and the dynamics are dependent upon the incident material and the bias voltage. For a single pulse the gas desorbed and ionized reached $87 \%$ of the total $\mathrm{K}^{+}$ion current for the stainless steel target by the end of the pulse and $98 \%$ for the copper target. The measured secondary emission coefficient for the stainless steel target was 10.87 and was 9.18 for copper. For each of the targets the total ionized gas and electron densities approached the beam density.

A successful correction of the beam centroid has been demonstrated with dipole magnets using a Jacobian based method. An analytic method was developed to examine the offsets of individual solenoids and quantify their individual and total contributions to the centroid motion. This has the potential to simplify the correction process and provide a better understanding of the expected centroid motion for different lattice tunes. 


\section{Chapter 6}

\section{Beam focusing and bunching}

A portion of the material in this chapter was also published in the 2007 Proceedings of the Particle Accelerator Conference [11]. Here we present studies of combined transverse and longitudinal focusing of a $0.3-\mathrm{MeV}, 26-\mathrm{mA} \mathrm{K}^{+}$ion beam. All of the experiments discussed in this chapter were conducted on the experimental configuration shown below in Fig. 6.1.

The experiment consists of the same high-current injector and four-solenoid matching section described in Chapter 5. Just downstream of the transport lattice the beam enters an induction bunching module (IBM) where a velocity tilt is applied to axially compress the beam. Just downstream of the IBM, a suppression electrode and magnetic dipole are used to prevent plasma electrons from backstreaming into the induction gap and transport lattice. The beam space charge is neutralized $28 \mathrm{~cm}$ downstream of the IBM to optimize transverse focusing.

The first experiments described are those conducted to demonstrate neutralized transverse focusing. Next, we give a description of neutralized axial compression 


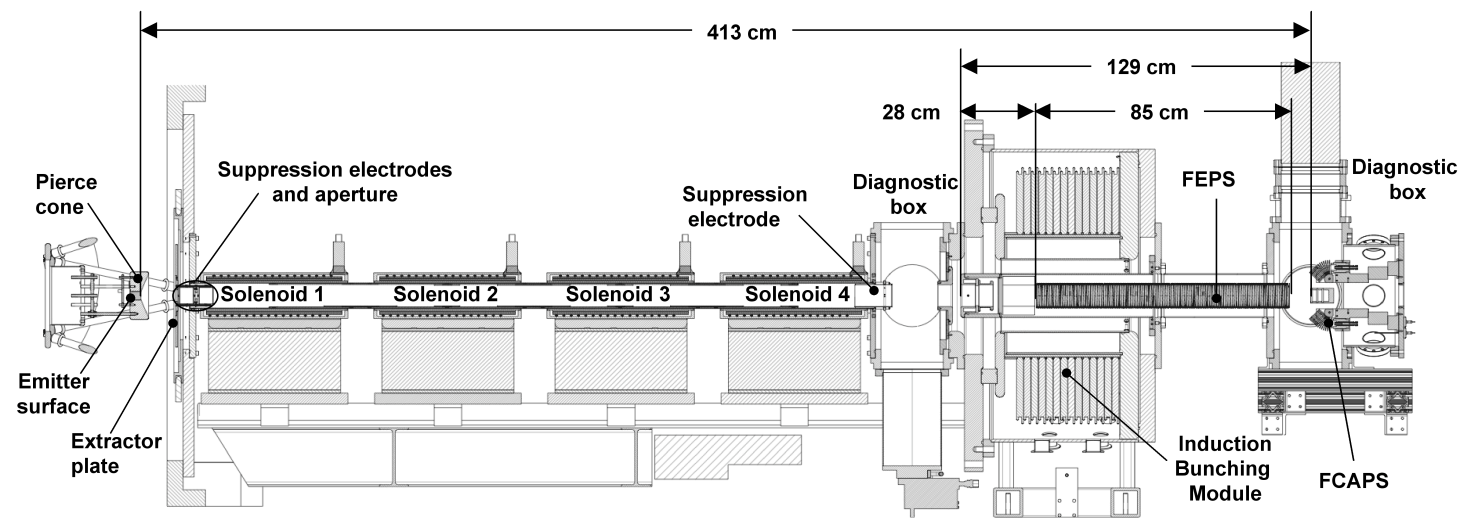

Figure 6.1. Elevation view of the Simultaneous Focusing Experiment on NDCX.

measurements and finally we show simultaneous longitudinal bunching and transverse focusing.

\subsection{Transverse focusing}

Transverse focusing experiments with a neutralizing background plasma are discussed in this section. Once a charged beam bunch is matched and transported through a focusing lattice it can be focused to a spot limited by its transverse envelope at the exit of the focusing lattice. The transverse envelope equation (Eq. 2.37) can be solved to determine the axial location of the beam waist downstream.

\subsubsection{NDCX Plasma sources}

A background plasma neutralizes the beam space-charge so that only the transverse emittance of the beam physically limits the beam waist. The plasma sources must meet certain criteria; the plasma must be cold $\left(\mathrm{T}_{\mathrm{e}} \sim 10 \mathrm{eV}\right)$ and the electron density $n_{e} \geq n_{b}$ (beam density). $n_{e}$ must be uniformly distributed or atleast $>n_{b}$ 
everywhere. If $n_{\mathrm{e}}$ falls off steeply in any direction before the beam reaches its desired focus the beam will be focused to a non-uniform distribution. In addition to the requirements listed above, during the operation of these plasma sources a vacuum pressure $>10^{-6}$ torr must be maintained. In the interest of this thesis we will only present the two types of plasma sources capable of fulfilling these requirements used on the NDCX.

\section{Ferroelectric plasma source (FEPS)}

Most of the neutralized drift length is provided by a ferroelectric plasma source (FEPS). Ferroelectric materials have proven to be high-current density electron emitters $[15,119-121]$ and can be used as large surface area, high-current density cathodes. Using a stainless steel mesh electrode on the emitting side of the ferroelectric material and a copper plate electrode on the back surface a capacitive discharge can be formed between the two electrodes to yield a plasma (Fig. 6.2). The voltage applied to the electrodes depends upon the thickness of the ferroelectric material to generate the discharge. In these experiments the material was $6.35 \mathrm{~mm}$ thick and the voltage necessary for a discharge was typically in the range of $7-10 \mathrm{kV}$. Plasma emission is observed and is simply explained by electron emission from the gaps between the dielectric surface and the edge of the metal electrode surface.

The dielectric constant is the key factor for this configuration. Ferroelectric materials have extremely large dielectric constants, in this case $\mathrm{BaTiO}_{3}$ has a dielectric constant in the range of 1000-3000. Once the threshold voltage is reached plasma is formed over the entire surface of the dielectric. Typical current density yields are 0.5 $\mathrm{A} / \mathrm{cm}^{2}$. Plasma emissions from these dielectrics have been characterized for $\mathrm{BaTiO}_{3}$; there is a sharp fall off in electron density from the dielectric surface as a function of distance. The velocity of the plasma ions moving away from the dielectric surface is $\sim 1 \mathrm{~cm} / \mu \mathrm{s}$. 
The plasma is essentially all metal so the neutrals stick to the walls of the vacuum system and do not result in too much pressure rise. The exact composition and charge state of the plasma is unknown and is most likely a mix of several ion species [123].

The features of this plasma source are exactly what is required for neutralization of the beam space charge in the NDCX. Furthermore, the ability to make the plasma emitting layer arbitrarily long is important for the drift lengths used $(>1 \mathrm{~m})$ in the NDCX. The structure used has an inner diameter of $76.2 \mathrm{~mm}$ (radius $38.1 \mathrm{~mm}$ ). This small tube diameter allows plasma densities on axis to climb above $10^{10} \mathrm{~cm}^{-3}$. Typical plasma temperatures range from $10-30 \mathrm{eV}$ for peak plasma densities.

An 85-cm long plasma source was fabricated for the NDCX in 5 individual sections that were 15-20 cm long. These sections were built with cylindrical ferroelectric pieces. The ferroelectric cylinders were $25.4 \mathrm{~mm}$ long $\times 6.35 \mathrm{~mm}$ thick [Fig. 6.2(a)]. The front surface electrode consisted of a stainless steel mesh and $36,0.25 \mathrm{~mm}$ stainless steel wires strung along the length of the cylinder. The wires were fastened to aluminum rings at each end of the source with 36 set screws [Fig. 6.2(b)]. The wires were pulled tight to hold the ferroelectric cylinders firmly together. Each aluminum ring was mounted in a Delrin insulating sleeve to isolate it electrically from the copper jacket (outer surface electrode). The high-voltage pulse is applied to the outer copper jacket and the aluminum rings and stainless steel wires are grounded [Fig. 6.2(c)].

The power supply used to pulse this source is a capacitor bank with a pulseforming network to match the impedance of the source and maintain the microsecond pulse shapes shown in Fig. 6.3. As presently configured the pulse-forming network is matched to $4 \Omega$ and has an output of $>10 \mathrm{kV}$ and $2 \mathrm{kA}$. Thyratrons control the discharge of the charging capacitors. The output of the power supply is two $3-\mu \mathrm{s}$ pulses with an adjustable time delay between the pulses. One voltage pulse drives FEPS sections 1 and 4 and the other drives sections 2, 3, and 5 (Fig. 6.3). 


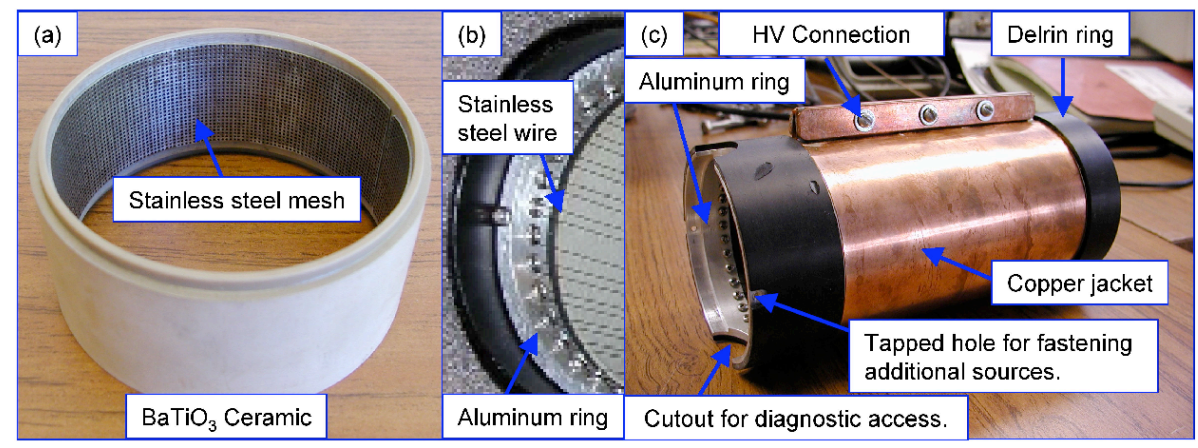

Figure 6.2. Photograph of (a) a single ferroelectric cylinder; (b) closeup of the stainless steel wires fastened to the aluminum ring and; (c) an individual section of the FEPS with parts labeled.
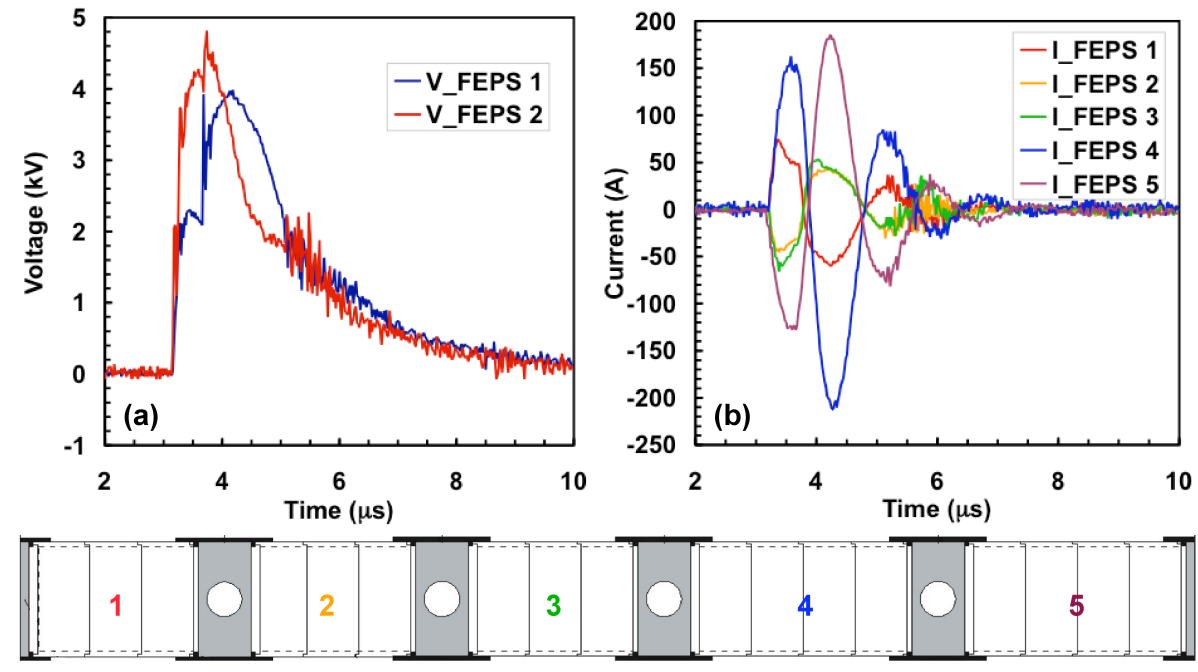

Figure 6.3. Measured (a) voltage waveforms and; (b) current waveforms when operating the 5 sections (shown below) of the FEPS at $9 \mathrm{kV}$. The 5 sections of the FEPS are shown below. 


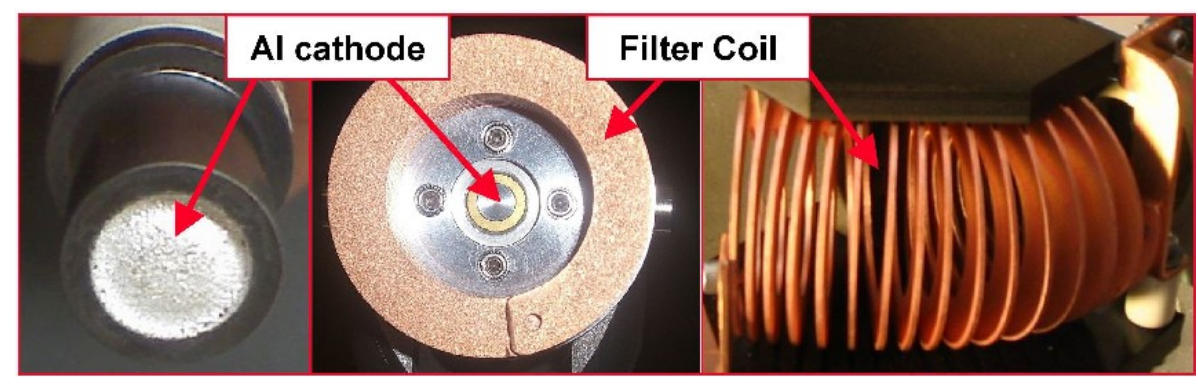

Figure 6.4. Photograph of an aluminum cathode source closeup (left); inside assembled source (center) with a copper filter coil (right and center).

\section{Filtered cathodic arc plasma source (FCAPS)}

Two filtered cathodic arc plasma sources (FCAPS) were also used in the system as an additional source of electrons to neutralize the beam space charge at the diagnostic plane [16]. The plasma is generated by a high-voltage arc discharge at an aluminum cathode surface (Fig. 6.4). The metal is vaporized into macroparticles, some of which become ionized. The filter surrounding and just downstream of the cathode (Fig. 6.4) is used to filter out the neutral macroparticles and help confine the plasma ions and electrons. The filter itself creates a magnetic guide field $\sim 1 \mathrm{kG}$ for confinement. The FCAPS used on NDCX were separated by $13 \mathrm{~cm}$ and each filter coil had an inner diameter of $2.36 \mathrm{~cm}$ and an outer diameter of $4.72 \mathrm{~cm}$. At the cathode surface plasma densities and temperaures approach $10^{16} \mathrm{~cm}^{-3}$ and $5 \mathrm{eV}$. At the exit of the filter the plasma densities and temperaures typically fall below $10^{14} \mathrm{~cm}^{-3}$ and $3 \mathrm{eV}[16]$.

The high voltage was provided by a conventional $2 \mathrm{kV}$ power supply, which charges the capacitors in a pulse-forming network (PFN). The PFN used was designed to drive the two FCAPS to a maximum current of $500 \mathrm{~A}$ at $2 \mathrm{kV}$ [124]. A schematic of the electrical configuration used to drive the FCAPS is shown in Fig. 6.5. The resulting current pulse shape was a $400 \mu$ s pulse with flattop $>200 \mu$ s (Fig. 6.6). 


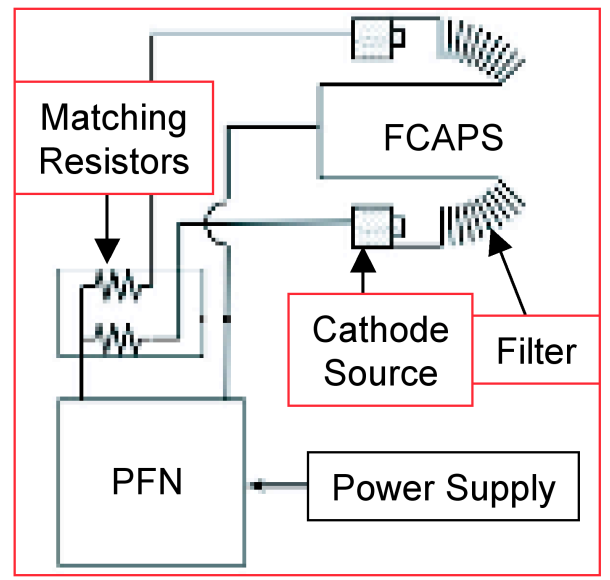

Figure 6.5. Schematic of FCAPS electrical configuration.

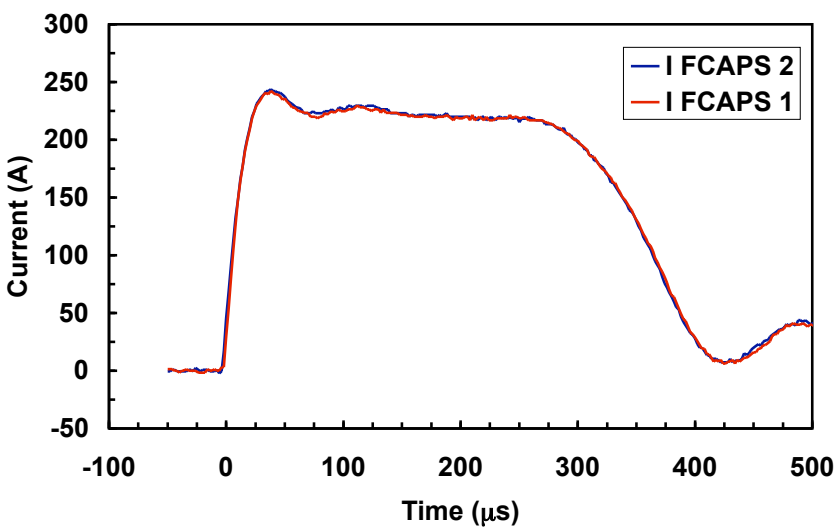

Figure 6.6. Measured current waveforms when operating the FCAPS at $1 \mathrm{kV}$. 

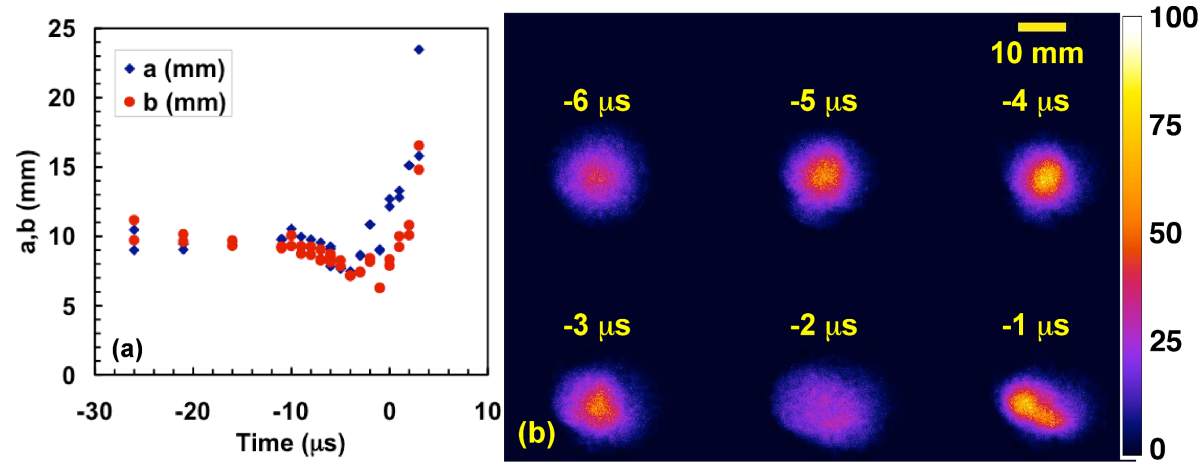

Figure 6.7. Neutralized (a) beam radii and; (b) transverse distributions J(x,y) at the scintillator plane $(\mathrm{z}=413 \mathrm{~cm})$ versus FEPS time.

\subsubsection{Measurements of neutralized ballistic focusing}

The 85-cm long FEPS was installed $28 \mathrm{~cm}$ downstream of the induction gap (Fig. 6.1). Experiments were conducted to optimize neutralization of the beam space charge with the FEPS without longitudinal focusing. Scans of the FEPS delay time relative to the beam time in the plasma channel were performed. Monitoring the transverse distribution $[\mathrm{J}(\mathrm{x}, \mathrm{y})]$ of the beam it was discovered that firing the FEPS $4 \mu$ s before the beam entered the plasma channel minimized the spot size of the beam [Figs. 6.7(a) \& (b)]. The envelope for this experiment was not optimized for transverse focusing of the beam to an emittance limited spot at the diagnostic plane. Nonetheless the results complement conclusions drawn from previous plasma temperature and density measurements and simulations $[123,125]$. The measured time for the electrons from the FEPS to maintain a cool $\left(\mathrm{T}_{\mathrm{e}}<30 \mathrm{eV}\right)$ and sufficiently high $\mathrm{n}_{\mathrm{e}}$ was close to $4 \mu \mathrm{s}$.

Neutralization does not begin until operating the source above $7 \mathrm{kV}$, and $8-9 \mathrm{kV}$ is the optimum operating point depending on the lifetime of the source. Since many of the cylinders are driven in parallel, those having experienced more discharges have higher breakdown thresholds. Once the minimum expected neutralized spot (based on 

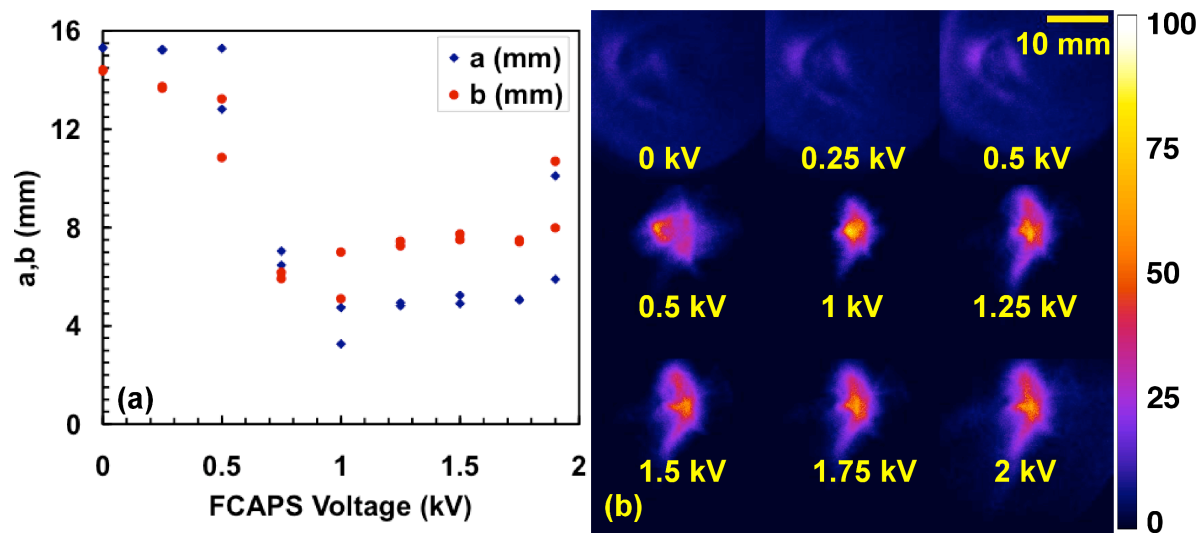

Figure 6.8. Neutralized (a) beam radii and; (b) transverse distributions $J(x, y)$ at the scintillator plane $(\mathrm{z}=413 \mathrm{~cm})$ versus FCAPS voltage.

envelope calculations) was obtained further increases in voltage were not attempted due to the possibility of failure of newer cylinders with lower breakdown thresholds.

Experiments were also conducted to optimize neutralization of the beam with the FCAPS without longitudinal focusing. Again scans in time were made relative to the beam time in the plasma channel. Monitoring the transverse distribution $[\mathrm{J}(\mathrm{x}, \mathrm{y})]$ of the beam it was discovered that firing the FCAPS about $30 \mu$ s before the beam entered the plasma channel minimized the spot size of the beam. This delay corresponded to the rise time of the FCAPS pulse and the time at which the plasma density peaks providing a better operating point for the plasma source [126].

The voltage threshold necessary for consistent discharges and neutralization with the FCAPS was about $750 \mathrm{~V}$. Plasma density increases linearly with discharge voltage [126], although there appears to be no improvement in neutralization of the beam space charge above $1 \mathrm{kV}$ (Fig. 6.8). This could be explained by an increased electron temperature and energy, if the electrons are too energetic they will not neutralize the beam space charge potential effectively. 


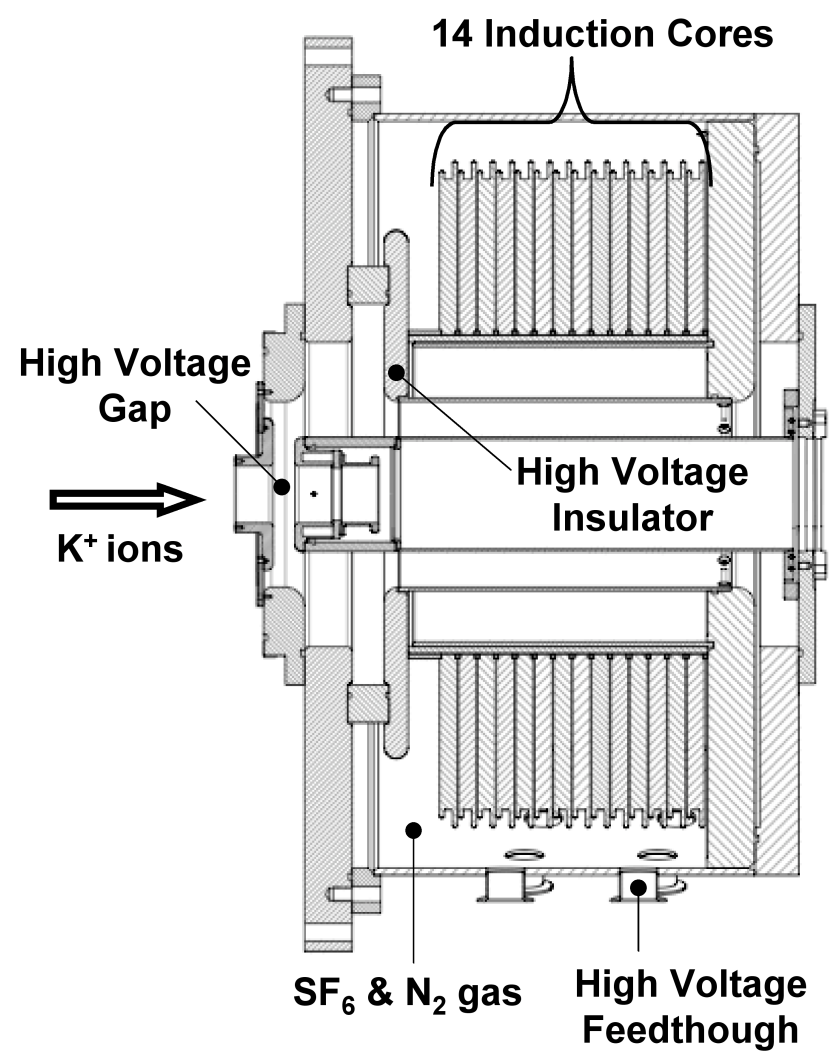

Figure 6.9. Cross section of the IBM used on NDCX.

\subsection{Axial compression}

\subsubsection{Induction Bunching Module (IBM)}

An induction bunching module (IBM) was used to apply the velocity tilt on the NDCX. The IBM is an induction accelerator cell, which is tuned primarily to only axially compress a beam bunch rather than accelerate it in parallel. The IBM used for NDCX consisted of 14 individual induction cores (ferro-magnetic rings) only 12 of which were driven due to limitations in the pulse forming network (Fig. 6.9). The cores are housed in a pressurized gas vessal composed of a mixture of $33 \% \mathrm{SF}_{6}$ and $66 \% \mathrm{~N}_{2}$, which is separated from the vacuum by a ceramic high voltage insulator. 


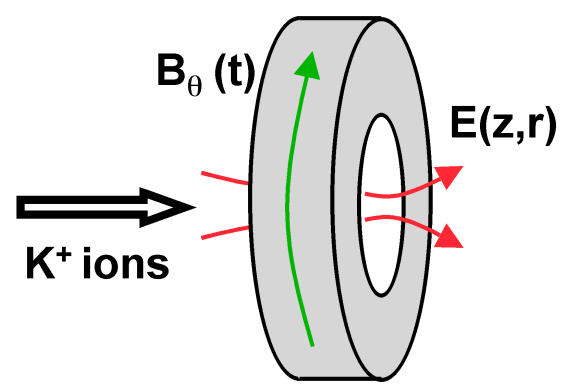

Induction Core

Figure 6.10. Cartoon of the operation of an induction cell.

Each core receives a high voltage pulse from a thyratron-switched modulator, which generates a time dependent azimuthal magnetic flux. From Faraday's Law or Maxwell's first equation (Eq. 2.2a) an electric field is generated across a high voltage gap (Fig. 6.10). Six of the cores are pulsed with a positive potential and the remaining six are pulsed with a negative potential and add inductively at the acceleration gap. After arranging the time delays of the individual pulses a linear velocity ramp can be obtained. The time delay for each pulse is unique to a velocity tilt for a particular beam energy and focal length as explained in Section 2.4.3.

The IBM provided a linear velocity $\operatorname{ramp}(\Delta \mathrm{v} / \mathrm{v} \approx \pm 15 \%)$ on a $200 \mathrm{~ns}$ portion of the injected beam and was tuned specifically for a beam energy of $300 \mathrm{keV}$ and a drift distance of $1.29 \mathrm{~m}$ (Fig. 6.1). The IBM was tuned to best match the ideal velocity tilt (Fig. 6.11). Slight improvements have been made in the resulting waveform although identical hardware was used for the IBM in recent experiments compared to that reported by Roy et al., in 2005 [9]. Examining the voltage difference from the ideal case over the relevant portion of the injected waveform $(0.1<\mathrm{t}<0.25 \mu \mathrm{s})$ shows that the new waveform has an average voltage difference of $<0.5 \mathrm{kV}$ compared to $>1.5 \mathrm{kV}$ in 2005 [Fig. 6.11(b)]. This effective shift can be tuned out, however both pulses show an oscillation even after filtering. This oscillation is an imperfection in 

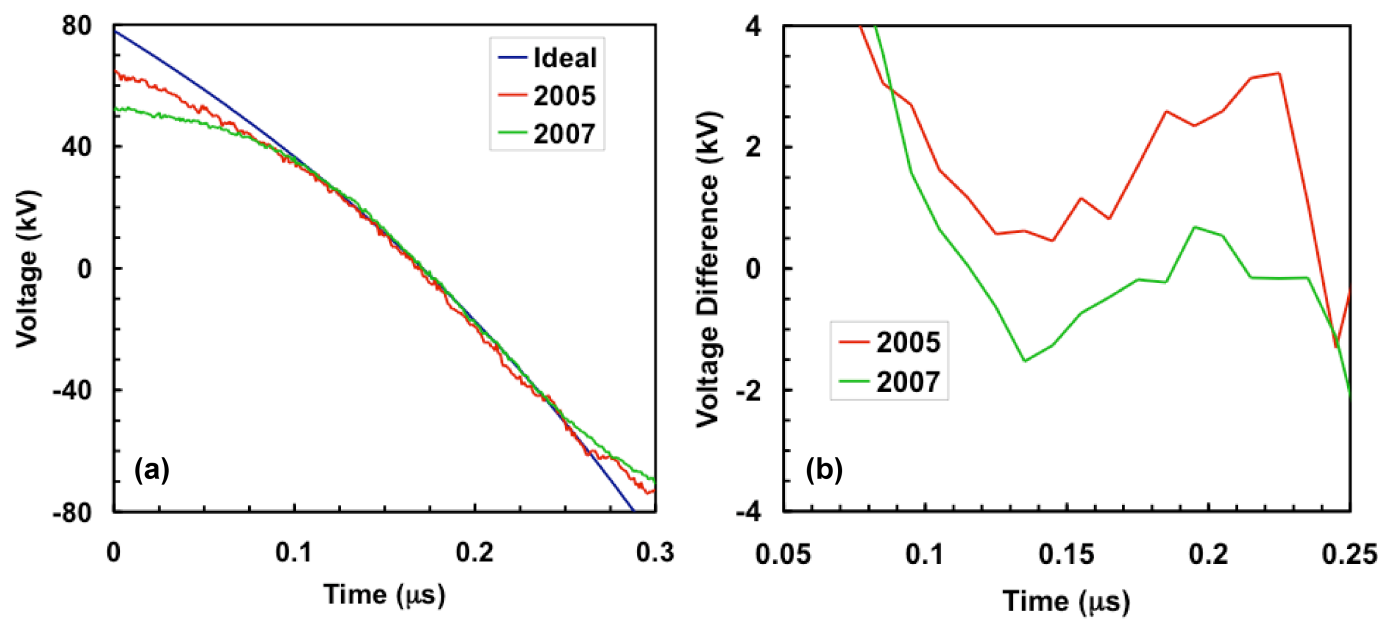

Figure 6.11. (a) IBM waveform comparison to ideal case for 2005 and 2007; (b) improvement in voltage difference (filtered for clarity).

the waveform which reduces the uniformity of the beam adding larger pulse widths and possibly a pedestal at the base of the peak compressed pulse.

\subsubsection{Compression ratio}

A description of axial compression measurements with the fast pinhole Faraday cup (FFC) and phototube (PT) (explained in Sections 3.1.2 \& 3.1.3) is shown in the next few sections. First, the compression ratio (R) quantifies the current amplification. It is the ratio of the voltage of the compressed signal $\left(\mathrm{V}_{\mathrm{f}}\right)$ to the voltage of the uncompressed signal $\left(\mathrm{V}_{\mathrm{o}}\right)$ recorded by the beam diagnostic. Both of these values are extracted after performing a background subtraction, described in Section 3.7.1. $\mathrm{V}_{\mathrm{f}}$ is the peak voltage value and $V_{\mathrm{o}}$ is the average of the uncompressed voltage signal at the relevant time frame on the diagnostic. The relevant time frame is dependent upon how much of the beam is compressed or perturbed by the IBM waveform. For the data presented below this time frame was $t \pm 100$ ns about the time of the peak beam current. If the signal to noise ratio is low, $\mathrm{V}_{\mathrm{o}}$ should be compared and averaged 
over several shots for this $200 \mathrm{~ns}$ interval. The more shots the better because the random noise will decrease like $\mathrm{N}^{1 / 2}$, where $\mathrm{N}$ is the number of shots, assuming negligible systematic drifts. However, 3-5 shots are sufficient since they have a standard deviation $<0.1 \mathrm{mV}$ or $<1 \%$, which is less than the variation in the Marx voltage in the diode.

Once $V_{f}$ and $V_{o}$ have been obtained the compression ratio can be found. These measurements must be taken for each beam energy setting since the voltage signal in the diagnostic (current) varies as $\mathrm{I} \sim \mathrm{V}^{3 / 2}$, where $\mathrm{V}$ is the Marx voltage used in the diode to extract the beam.

Quantifying the compression ratio measured by the phototube requires uniform light collection efficiency. To date, the light collection efficiency is poor and is spatially dependent as explained in Section 3.1.3. Thus, $\mathrm{R}$ has not been extracted from the phototube data.

\subsubsection{Compressed pulse width}

The next quantity, the pulse width, is a complimentary measurement of current amplification or bunching. The minimum measured compressed pulse width is limited by the time response of the diagnostic as explained in Sections 3.1.2 \& 3.1.3.

Calculating the compressed pulse width can be done two separate ways:

1. The Tektronix scope model TDS $654 \mathrm{C}$ used for these experiments measures the burst width, and positive and negative widths of the compressed pulses. The measurements are interpolations from the time at the peak value to the two values nearest the half amplitude. This interpolation yields a full width at half maximum (FWHM) of the compressed pulse width. For a 1 and $2.5 \mathrm{GHz}$ sam- 
pling the minimum resolvable pulse width is $>1 \mathrm{~ns}$ and $>400$ ps respectively. This can also be calculated from the data points of saved waveforms and yield identical results.

2. A Gaussian can also be fit to the data points. A comparison of the fitted FWHM $=2 \sqrt{2 \ln (2)} \sigma$ to the burst width, gives similar results.

\subsubsection{Normalized beam current}

The final quantity measured is the actual current. Extracting the beam current from the pinhole Faraday cup is based on calibration versus a standard Faraday cup. The most unambiguous way to do this is to place both diagnostics at the same diagnostic plane and measure the voltage signal from the beam with both diagnostics. The beam must be smaller than the accepting area of both diagnostics. The background subtraction must be performed first. Next, the voltage signal for both diagnostics is integrated to find the total number of volt seconds over the beam pulse. The normalization factor (scale factor) is the ratio of integrated signal in the standard Faraday cup to the integrated signal in the pinhole Faraday cup. This normalization approach was carried out and yielded a factor of $41.78 \pm 1.90$ (Fig. 6.12).

\subsubsection{Suppressor (middle plate) signal without axial com- pression}

When examining the FFC it is also worth noting the signal on the middle plate or electron suppressor of the diagnostic (Fig. 6.13). First we examine the suppressor signal without axial compression. Under normal operating conditions the middle plate does accumulate charge. The typical bias voltage on the middle plate is $-200 \mathrm{~V}$, 

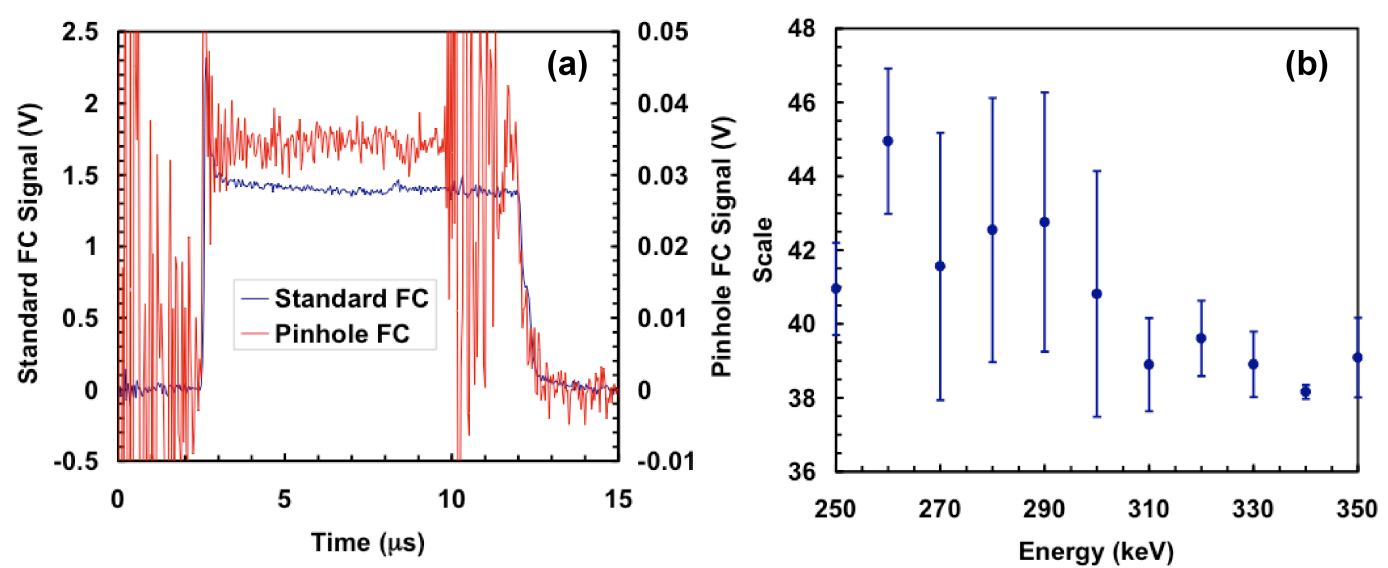

Figure 6.12. (a) Measured reduction in ion current signal due to pinholes (red) compared to the signal in a standard Faraday cup; (b) Average scale factor as a function of energy for three consecutive shots.

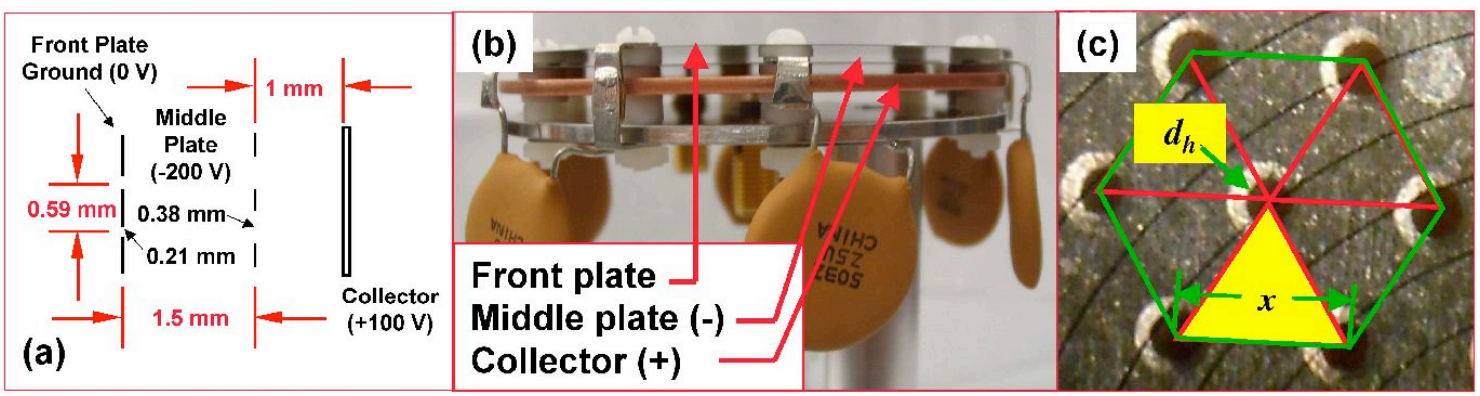

Figure 6.13. Schematic of the (a) pinhole Faraday cup geometry; (b) photograph of the constructed pinhole Faraday cup and; (c) close-up of the pinhole pattern.

and $+100 \mathrm{~V}$ on the collector. Based on electron cloud effects with intense ion beams it is known that gas will be desorbed when the beam is normally incident upon a surface and will be ionized by incoming beam ions (Chapter 6) $[12,13]$.

We have observed a linearly increasing positive current collected on the middle hole plate [Fig. 6.14(a)], with a peak current of nearly $3 \mathrm{~mA}$ at the end of a 10- $\mu \mathrm{s}$ beam pulse. This signal amplitude is similar to the $\mathrm{K}^{+}$flux incident on the collector of the Faraday cup. The linear time dependence is suggestive of a gas-buildup and ionization mechanism, and should not be confused with beam ions striking the middle 

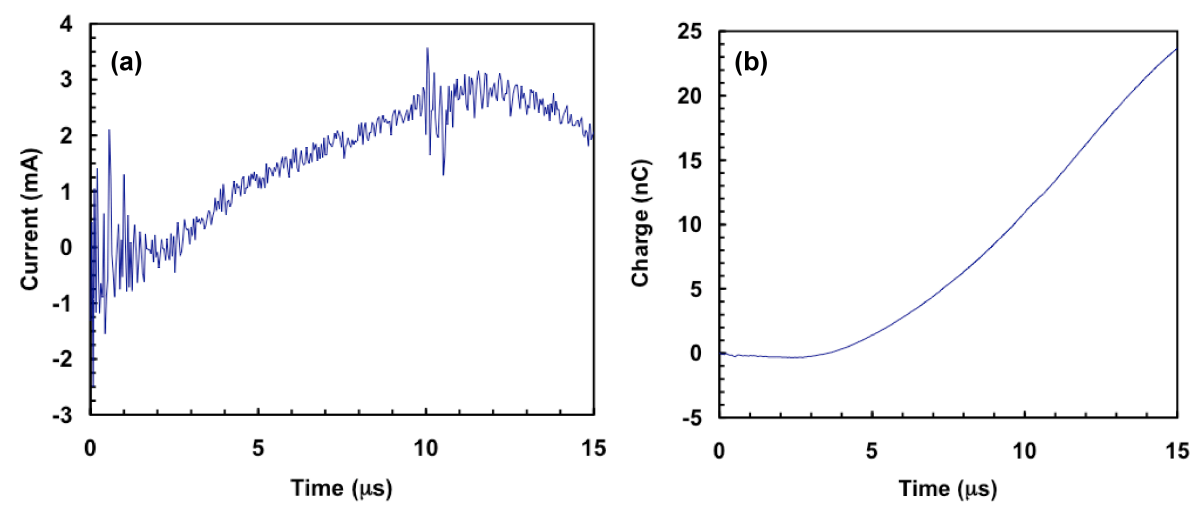

Figure 6.14. (a) Measured current and (b) charge collected on suppressor (middle plate) without current density compression for a $10-\mu$ s beam pulse.

plate (which is unlikely due to the achieved alignment tolerances). $\mathrm{K}^{+}$ions in this energy range desorb $3 \times 10^{3} \mathrm{H}_{2}$ molecules $/ \mathrm{K}^{+}$for normal incidence on stainless steel [58]. The gas (predominantly $\mathrm{H}_{2}$ ) expands into the beam path and becomes ionized by the incoming beam at a rate that is proportional to the square of the transmitted beam current $\left(\mathrm{I}_{\mathrm{b}}^{2}\right)$. The relevant cross section is the sum of the ionization cross section and the charge-exchange cross section, which is $\sim 1 \times 10^{-15} \mathrm{~cm}^{2}$ according to measurements and Slater, and Thomas-Fermi models [127]. Since the middle hole plate is negatively biased, most of the gas ions will be collected on this plate. Based on these assumptions, a simple one-dimensional analytic model predicts that a $3 \mathrm{~mA}$ current should be collected on the middle plate at the end of the beam pulse due to ionized gas, in good agreement with the observations in Fig. 6.14. Thus, this observation is understood from gas ionization.

This charge collected on the middle plate confirms there are gas effects and may explain the reduced signal on the collector. Ionized gas introduces electrons. If these electrons are made beyond the middle plate they could be collected on the collector reducing the overall beam signal giving a normalization factor (Section 6.2.4) > the geometric factor, f (Section 3.1.2). 


\subsubsection{Suppressor (middle plate) signal with axial compres- sion}

Now we analyze the suppressor signal with axial compression. A bipolar spike is measured on top of the linearly increasing positive current collected on the middle hole plate during all measurements with current density compression (Fig. 6.15). This spike is simply a capacitive image current due to the compressing beam passing through the holes of the plate. This is similar to the capacitive image currents seen on other cylindrical electrodes on the NDCX (Section 5.4.2) [12, 13].

A simple calculation is performed below to quantify the expected amplitude of this bipolar signal. Assume a $\mathrm{K}^{+}$ion beam bunch with an ion energy of $300 \mathrm{keV}$ and initial beam current, $\mathrm{I}_{\mathrm{o}}$, of $30 \mathrm{~mA}$ is axially compressed to a final current, $\mathrm{I}_{\mathrm{f}}$, of $3 \mathrm{~A}$ and final pulse width, $t_{f}$, of 2 ns. The final length of the bunch, $z_{f}=v_{z} t_{f}$, where $v_{z}$ is the axial beam velocity $(1.22 \mathrm{~mm} / \mathrm{ns})$ and $z_{f}=2.4 \mathrm{~mm}$. The thickness of the middle plate $z_{\text {plate }}=0.1 \mathrm{~mm}$. The image charge collected on the plate, $Q_{\text {plate }}$, due to the compressing beam bunch can be approximated as

$$
Q_{\text {plate }}=\frac{z_{\text {plate }}}{z_{f}} \frac{Q_{f}}{f},
$$

where $Q_{f}=6 \mathrm{nC}$ is the final charge of the bunch and $f$ is the geometric factor (8.7) defined in Section 3.1.2 (Eq. 3.1). This yields a $Q_{\text {plate }}=29 \mathrm{pC}$ and assuming this is evenly split as the compressing beam bunch enters and exits the plate the observed image current collected should be no greater $\pm 29 \mathrm{~mA}$. If we assume a positive image charge is seen as the compressing beam is traversing the gap between the front and middle plates (Fig. 6.13) and a negative image charge is seen as the compressing beam is traversing the gap between the middle plate and the collector then an image current no greater $\pm 20 \mathrm{~mA}$ should be collected should be on the middle plate. This is a rough estimate for the peak values of axial compression observed and would be 

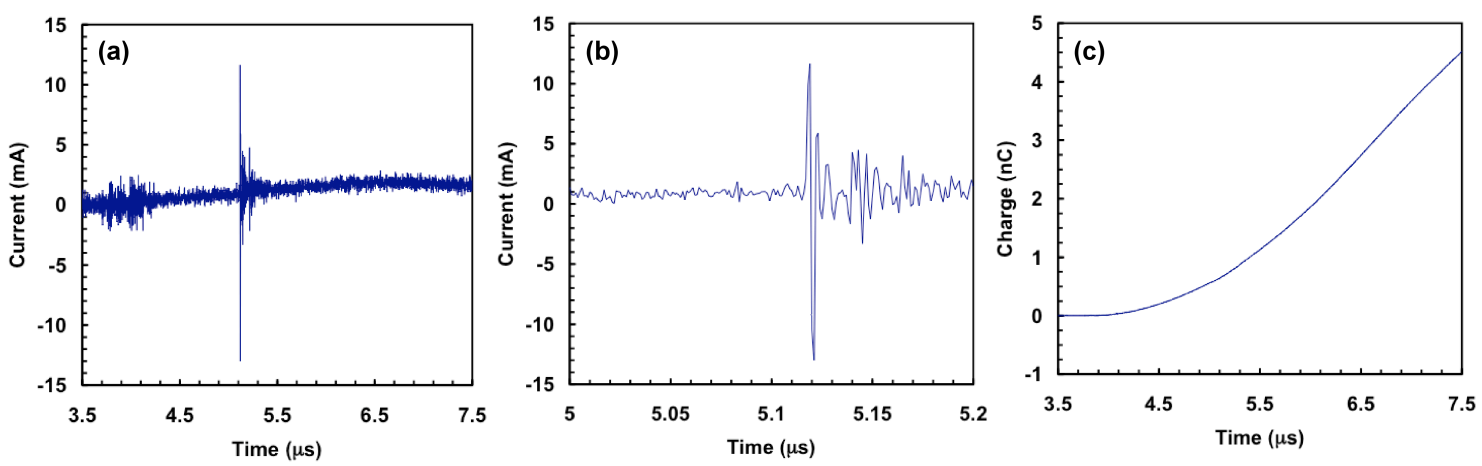

Figure 6.15. (a) Measured current; (b) zoomed in to view capacitive image current and; (c) charge collected on suppressor (middle plate) with current density compression for $3-\mu$ s beam pulse.

reduced for lower values of peak current. This estimate does agree fairly well with the measured image currents observed on the plate in Fig. 6.15.

\subsubsection{Measurements of neutralized axial compression}

If the beam space-charge is perfectly neutralized then only two factors limit the axial compression, the velocity tilt $\Delta \mathrm{v}_{\mathrm{o}}$ and the velocity spread $\Delta \mathrm{v}_{\mathrm{z}}$ of the uncompressed beam bunch (Section 2.4.3). Plasma neutralization begins $28 \mathrm{~cm}$ downstream of the IBM with an 85-cm long FEPS. The fully neutralized beam then drifts $16 \mathrm{~cm}$ to the focal plane. Two FCAPS are also used for neutralizing the beam at the diagnostic plane. The limitation of $\Delta \mathrm{v}_{\mathrm{z}}$ will be discussed in Chapter 7 .

Ignoring transverse focusing and assuming the transverse beam envelope is sufficiently small enough to both transport and diagnose the beam without scraping then axial compression measurements are feasible. For neutralized axial compression measurements to take place the beam must enter the IBM within a certain envelope range. The radius of the incoming beam and the convergence angle must be large enough to establish a converging beam at the entrance of the neutralization section 

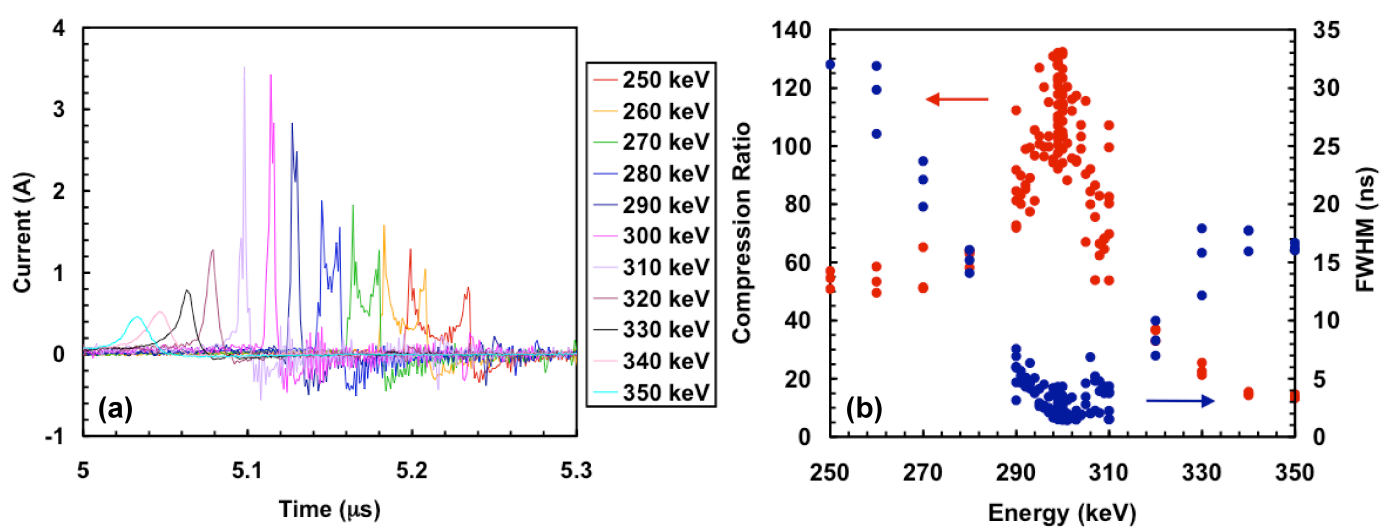

Figure 6.16. Energy dependence at the axial focal plane shown for (a) single shots and; (b) multiple shots at different beam energies.

with envelope parameters that focus the beam at the desired focal plane (diagnostic location). Typical beam radii are in the range of $10<\mathrm{a}(\mathrm{mm})<25$ with a convergence angles of $15<\mathrm{a}^{\prime}(\mathrm{mrad})<30$.

Measurements were made to determine the axial focus. Several data sets demonstrate the dependence of the axial focal plane on the beam energy, E (Fig. 6.16). The results of energy scans (variations of the Marx voltage) from 250-350 keV show the axial focus can be achieved with an $\mathrm{E}=300 \mathrm{keV}$ however there is a variance of about $10 \%$ in the compression ratio and a fluctuation of less than 1 ns in the FWHM at the focus. The variations are caused in part by voltage fluctuations of $5 \%$ and a timing jitter of $10 \mathrm{~ns}$ induced by the IBM.

These experiments demonstrate good agreement between the two different diagnostic techniques used to measure the axial focus of the ion beam, the FFC and the PT Both methods have demonstrated compressed pulse widths of $2.4 \pm 0.8$ ns at peak axial focus (Fig. 6.17). This pulse width corresponds to a compression ratio $>100$ (based on the fast Faraday cup), or a peak current > $2.6 \mathrm{~A}$; an improvement from previous experiments [9]. Slight improvements in the velocity tilt (Fig. 6.11) 

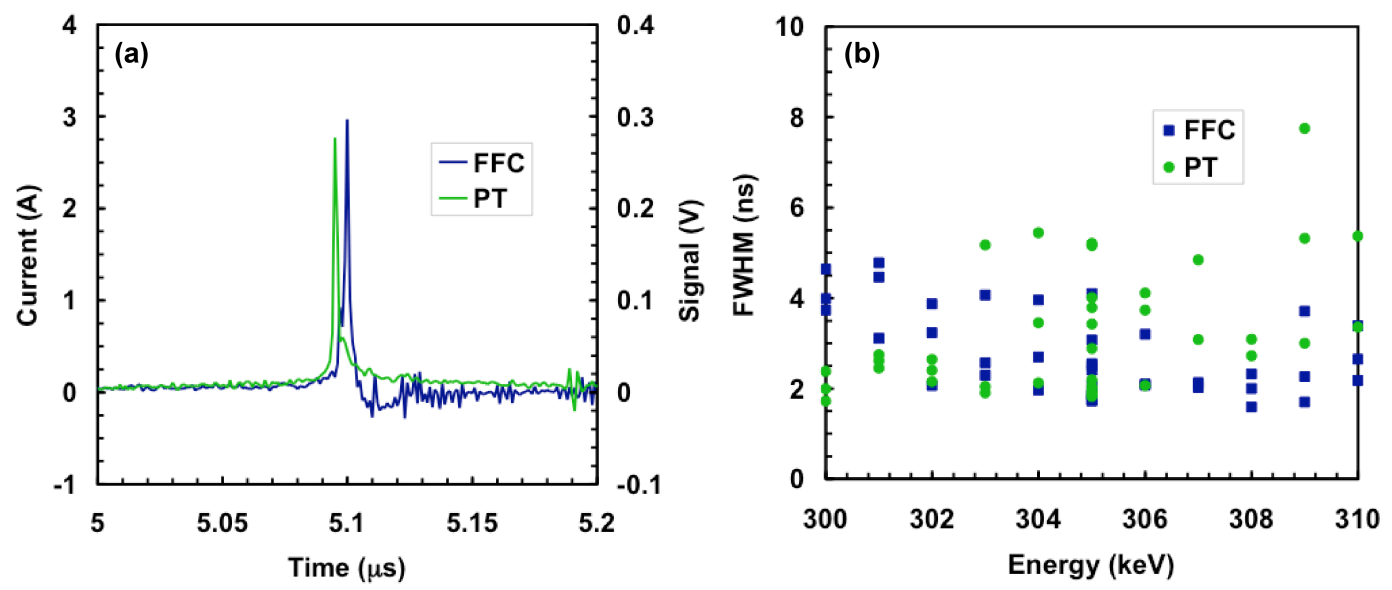

Figure 6.17. (a) Current waveform at the focal plane for a neutralized $\mathrm{K}^{+}$ion beam measured with the fast Faraday cup (FFC) (blue) and phototube (PT) (green) and; (b) pulse widths measured for multiple shots near the axial focal plane.

measurement techniques, and the use of the FEPS help explain this. The FCAPS, which was used solely in past measurements, makes a high velocity plasma that may be too energetic to effectively neutralize the beam space charge potential when used beyond the focal plane reducing the peak axial focus.

\subsection{Simultaneous longitudinal bunching and transverse focusing}

After demonstrating transverse and axial focusing individually, studies of combined transverse and longitudinal focusing of $0.3-\mathrm{MeV}, 26-\mathrm{mA}$ singly charged $\mathrm{K}^{+}$ion beam were conducted on the NDCX as shown in Fig. 6.1. The IBM was located downstream of a beam diagnostic box located at the exit of the four-solenoid lattice [13]. The four-solenoid transport lattice was used to match the beam to the desired envelope parameters $\left(\mathrm{a}=11 \mathrm{~mm}, \mathrm{a}^{\prime}=-36 \mathrm{mrad}\right)$ at the entrance to the IBM. The IBM provided a linear velocity $\operatorname{ramp}(\Delta \mathrm{v} / \mathrm{v} \approx \pm 15 \%)$ on a $200 \mathrm{~ns}$ portion of the 

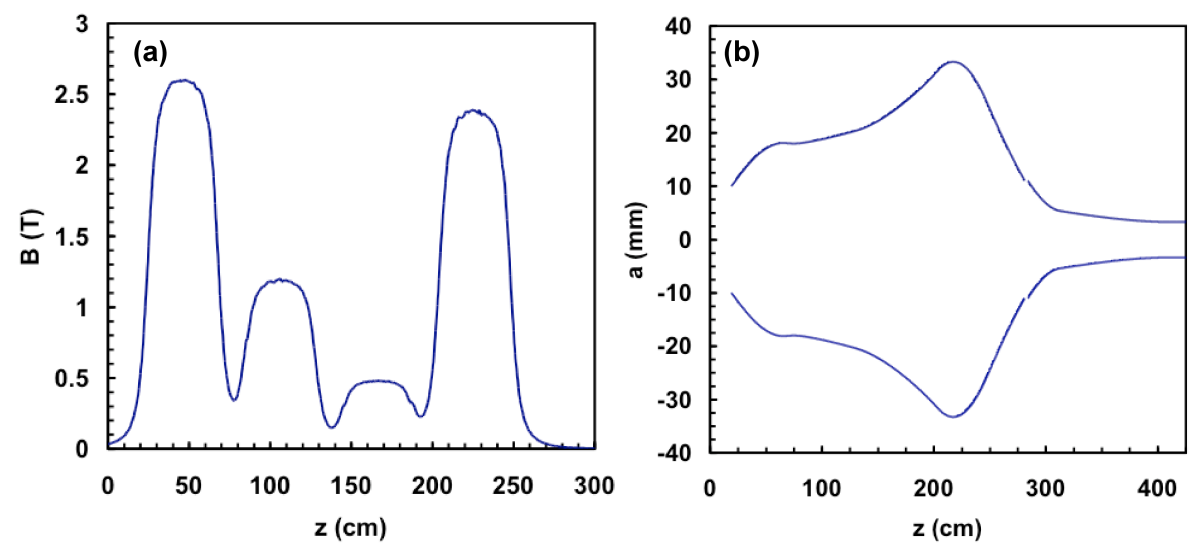

Figure 6.18. (a) Focusing lattice used to control: (b) the ideal beam envelope.

injected beam and was tuned specifically for the beam energy and a drift distance of $1.29 \mathrm{~m}$. Plasma neutralization began $28 \mathrm{~cm}$ downstream with an 85-cm long FEPS. The fully neutralized beam then drifted $16 \mathrm{~cm}$ to the focal plane. Two FCAPS were also used for neutralizing the beam at the diagnostic plane.

The defocusing effect, described in Section 2.5, was quantified and compensated for with a tune [Fig. 6.18(a)] where the beam envelope expands to $>30 \mathrm{~mm}$ in the fourth transport solenoid [Fig. 6.18(b)]. The maximum excursion of the beam was limited by beam halo, centroid offsets, the beam pipe radius (43 $\mathrm{mm})$, and the radius of the suppression electrode at the exit of the fourth solenoid (37 mm). Beam space charge and the radial defocusing effects provided by the velocity tilt required this steep convergence angle. Due to the suppression electrode and magnetic dipole (Fig. 6.1), plasma neutralization did not start until $28 \mathrm{~cm}$ downstream of the induction gap causing the beam to lose most of its convergence due to space charge. Given all of these constraints the minimum 2rms radius expected (based on envelope calcuations) with sufficient plasma neutralization was $3 \mathrm{~mm}$.

Although the transport solenoids and velocity tilt waveform had been tuned for 
a $300 \mathrm{kV} \mathrm{K}{ }^{+}$ion bunch with a focal length of $1.29 \mathrm{~m}$ it was expected that the beam envelope in the experiment might deviate by a few percent from the ideal calculation. There are two critical parameters for optimizing the envelope in the experiment. The first is the extraction voltage in the diode. Since, we have a constant perveance beam we can change the longitudinal envelope angle $\left[\mathrm{z}^{\prime}=\mathrm{z}\right.$ (bunch length before axial compression) / $\mathrm{f}$ (focal length)] by $<1$ mrad by varying the beam energy by 1 $\mathrm{keV}$. In order to maintain a constant beam envelope the field strength of the transport solenoids must be scaled by $\sqrt{\mathrm{E}}$ ( $\mathrm{E}$ is the beam energy). Once the operating points are determined for the axial focus, the transverse focus can be decoupled and optimized. Changes of just $0.02 \mathrm{~T}$ on a $2 \mathrm{~T}$ field make less than $1 \mathrm{mrad}$ changes in the transverse envelope angle; although this is small, the drift length of $1.65 \mathrm{~m}$ after the fourth transport solenoid acts as a long lever arm.

\subsubsection{Measurements of neutralized ballistic simultaneous fo- cusing}

After verifying the energy necessary to operate at axial focus (Section 6.2.7), measurements were then made to establish that the time dependent transverse focal plane coincides with the axial focus. Measurements of the transverse beam distribution $[\mathrm{J}(\mathrm{x}, \mathrm{y})]$ were made with a $100-\mu \mathrm{m}$ thick alumina scintillator and an image-intensified gated-CCD camera that imaged beam-induced light emission. The CCD was $512 \mathrm{x}$ 512 pixels with a resolution of 18 pixels/mm. A 10 ns gate width was used to capture the beam distribution at axial compression $(\mathrm{t}=5.095 \mu \mathrm{s})$ (Fig. 6.19). Larger gate widths (100 ns) were used 100 and $200 \mathrm{~ns}$ before and after the simultaneously focused spot to compensate for the reduced beam intensity and keep a satisfactory signal-tonoise ratio. A nearly three-fold reduction in the spot size for the compressed pulse is 


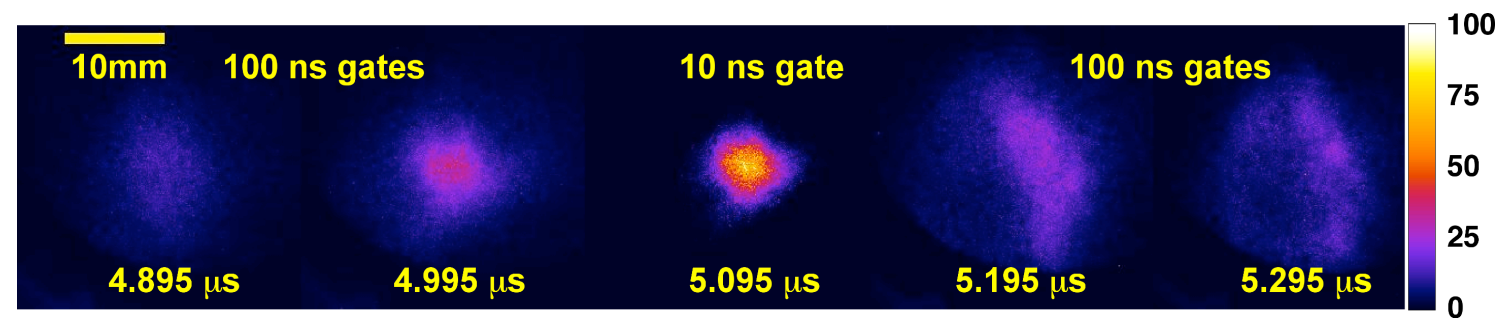

Figure 6.19. Time dependent transverse beam distributions demonstrating the simultaneous focal plane.

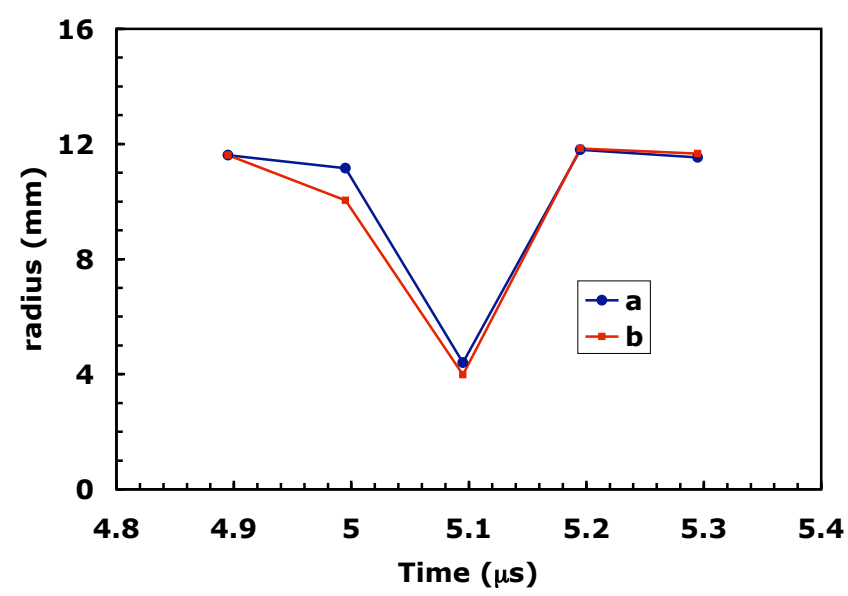

Figure 6.20. Projected spot radii (2rms) for distributions in Fig. 6.19.

shown in Fig. 6.20, where the horizontal and vertical 2rms radii (a and b) have been extracted from the images of Fig. 6.19.

A fully integrated source to target simulation was made using the LSP code [104]. A qualitative comparison of the measurements and the calculations in LSP of current density compression are shown in Fig. 6.21. The FWHM of the compressed current pulse calculated in LSP was $1.32 \mathrm{~ns}$ versus the $1.74 \mathrm{~ns}$ pulse measured for this particular shot in the experiment [Fig. 6.21(a)]. The structures of the two current profiles are qualitatively similar with the lower current shelves to the right and left of the peak current. This structure is most likely due to fringe field effects in the IBM and voltage imperfections in the Marx and IBM waveforms as described in Section 

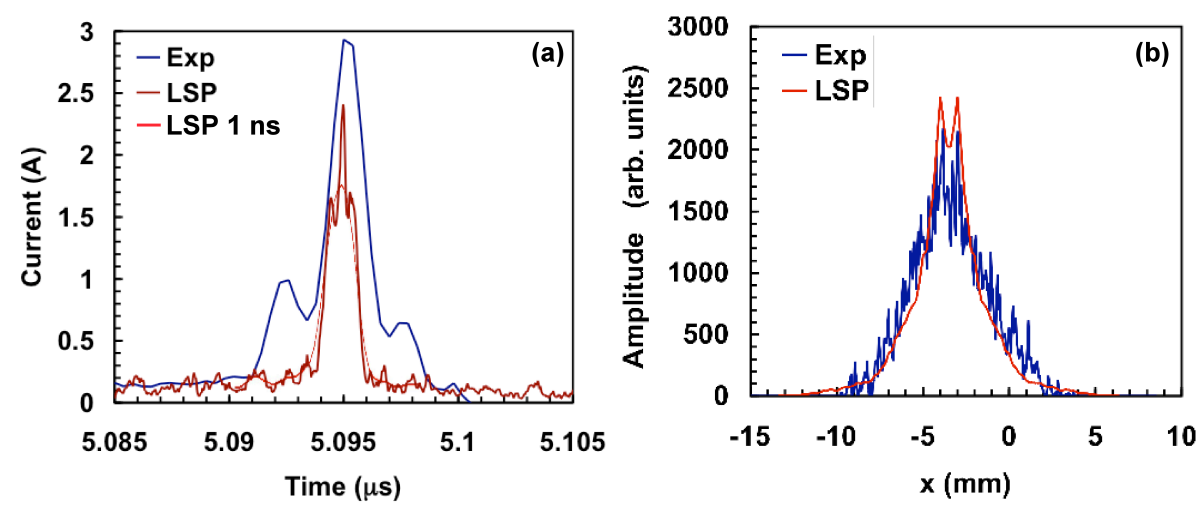

Figure 6.21. Comparison of experimental measurements on NDCX (blue) versus calculations made with LSP (maroon and red) at the focal plane: (a) compressed current profile with a 1-ns Gaussian response resolved from the raw LSP solution (red) and; (b) line-out of the transverse distribution shown in Fig. 6.19.

2.4.4. The structure is broadened in the measurement possibly because of the time resolution of the diagnostic. This measurement was made with the FFC, which has a minimum time resolution of $1.4 \mathrm{~ns}$. The structure of all of the peaks in the LSP calculation are all $<1 \mathrm{~ns}$ and are $>1 \mathrm{~ns}$ in the measurement. This indicates that our diagnostics cannot accurately resolve the structure of this measurement below $1 \mathrm{~ns}$ and there is a need for a faster diagnostic. The raw LSP solution was also convolved with a 1-ns Gaussian response to demostrate a signal we expect to measure based on simulations. The peak current is slightly reduced but the FWHM of the compressed pulse is $1.65 \mathrm{~ns}$ which is still slightly narrower than the experiment but it is well within the error bars of the measurement (0.8 ns).

Line-outs of the current density distributions measured in Fig. 6.19 and calculated in LSP were also made [Fig. 6.21(b)]. Although the LSP calculation has a smoother profile, the distributions agree qualitatively and have a double peaked structure at the center. The LSP calculation also has a narrower distribution and a FWHM of 2.5 $\mathrm{mm}$, which is nearly half the width of the measured value of $4.8 \mathrm{~mm}$.

Based on the measurements the beam density at the focal plane was $4.03 \times 10^{11}$ 
$\mathrm{cm}^{-3}$. With these results the peak beam intensity measured was about $4.8 \mathrm{~mJ} / \mathrm{cm}^{2}$ and calculated in LSP was $18.7 \mathrm{~mJ} / \mathrm{cm}^{2}$. The discrepancy between the two is nearly a factor four which is explained by the reduced FWHM calculated by LSP in Fig. 6.21(b). In the next section we will discuss plasma density measurements briefly which indicate the beam density may exceed the plasma density just a few $\mathrm{cm}$ upstream of the focal plane, reducing the maximum achievable intensity [128].

\subsection{Chromatic aberrations}

We know from Section 2.5 that the velocity tilt, which axially compresses the beam, also defocuses the beam transversely and causes a chromatic aberration. This is a time dependent effect that was examined using the numerical methods described in Sections 4.1.2 \& 4.1.3.

\subsubsection{Ballistic focusing}

The case for the experimental measurements above (Section 6.3.1) was examined to gain a better understanding of the beam envelope for the different energy slices and the expected composite energy distribution. As explained in Section 4.1.3 the individual energy slices are modeled as equal slices of charge from the initial (200 ns) bunch that is compressed.

The initial beam envelope parameters at the entrance of the IBM were $a=11$ $\mathrm{mm}$ and $\mathrm{a}^{\prime}=-36 \mathrm{mrad}$. Due to the defocusing effect the energy slice with no $\Delta \mathrm{v}_{\mathrm{z}}(\mathrm{E}$ $=300 \mathrm{keV}$ ) had a reduction in the convergence angle to $-32 \mathrm{mrad}$. The effect on the remaining portion of the bunch is shown in Fig. 6.22, the head of the beam bunch ( $\mathrm{t}$ $=0 \mathrm{~ns}$ ) gets decelerated and only has a slight change in envelope angle and the tail of 

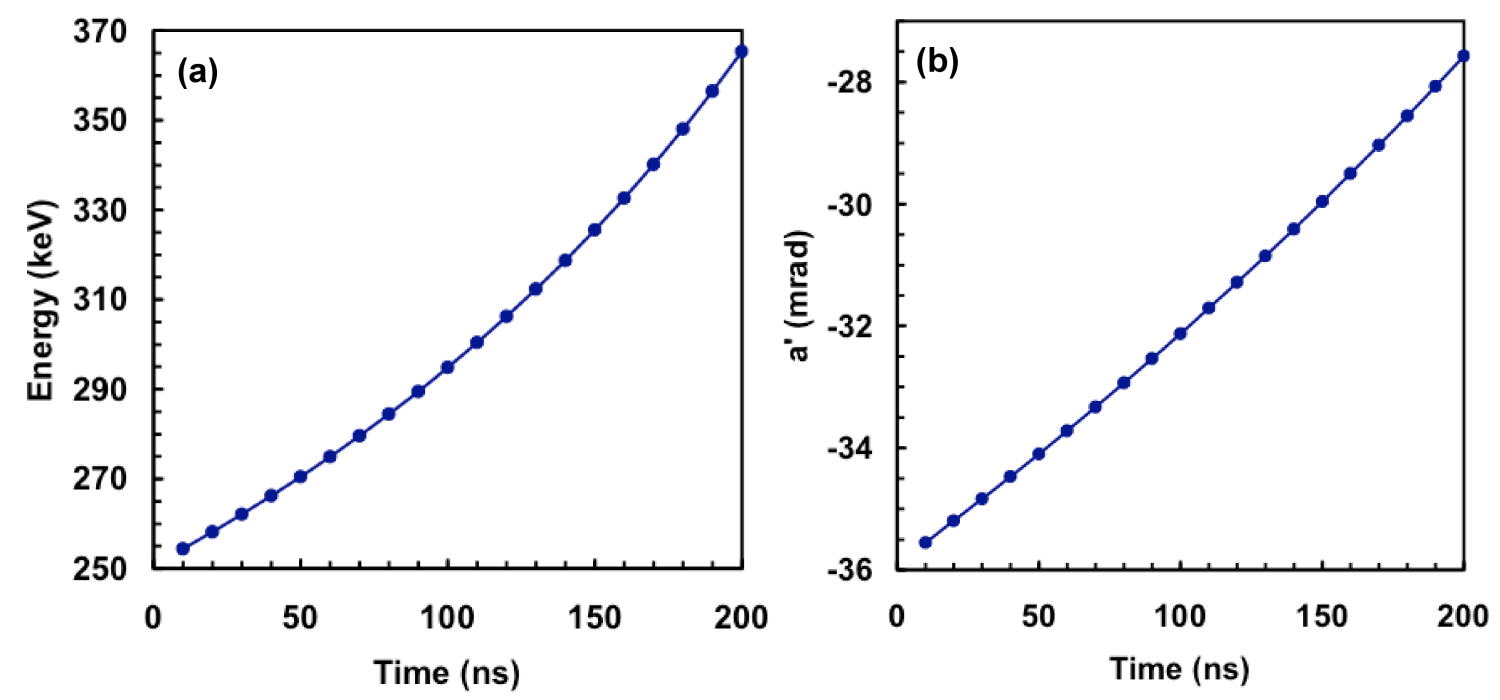

Figure 6.22. Calculated (a) change in beam energy and; (b) transverse envelope angle due to the velocity tilt.

the beam bunch $(\mathrm{t}=200 \mathrm{~ns})$ gets accelerated and has a decrease of almost 9 mrad in the convergence angle. There is a transit time effect through the induction gap that is ignored in these calculations which has an additional impact on the envelope angle of the particles closest to the head and tail. However, this effect has a small impact on the total energy deposition $\varepsilon$.

The energy slices near the head of the bunch $(\mathrm{E}<280 \mathrm{keV})$ have such a steep angle at the exit of the IBM $(\mathrm{z}=284 \mathrm{~cm})$ they actually come to a space charge dominated waist before entering the neutralization section $(\mathrm{z}=312 \mathrm{~cm})$ [Fig. 6.23(a)]. Once entering the neutralization section these lower energy slices $(\mathrm{E}<280 \mathrm{keV})$ have a diverging envelope. Energy slices with $\mathrm{E}>280 \mathrm{keV}$ become less converging due to the space-charge dominated drift region from $284 \geq \mathrm{z}(\mathrm{cm}) \geq 312$. So the highest energy slices near the tail of the bunch have the steepest convergence angle at the start of neutralization. This causes the highest energy slices to come to a focus upstream of the target $(\mathrm{z}=413 \mathrm{~cm})$ and the energy slice with no $\Delta \mathrm{v}_{\mathrm{z}}$ comes to a focus on target as designed. 

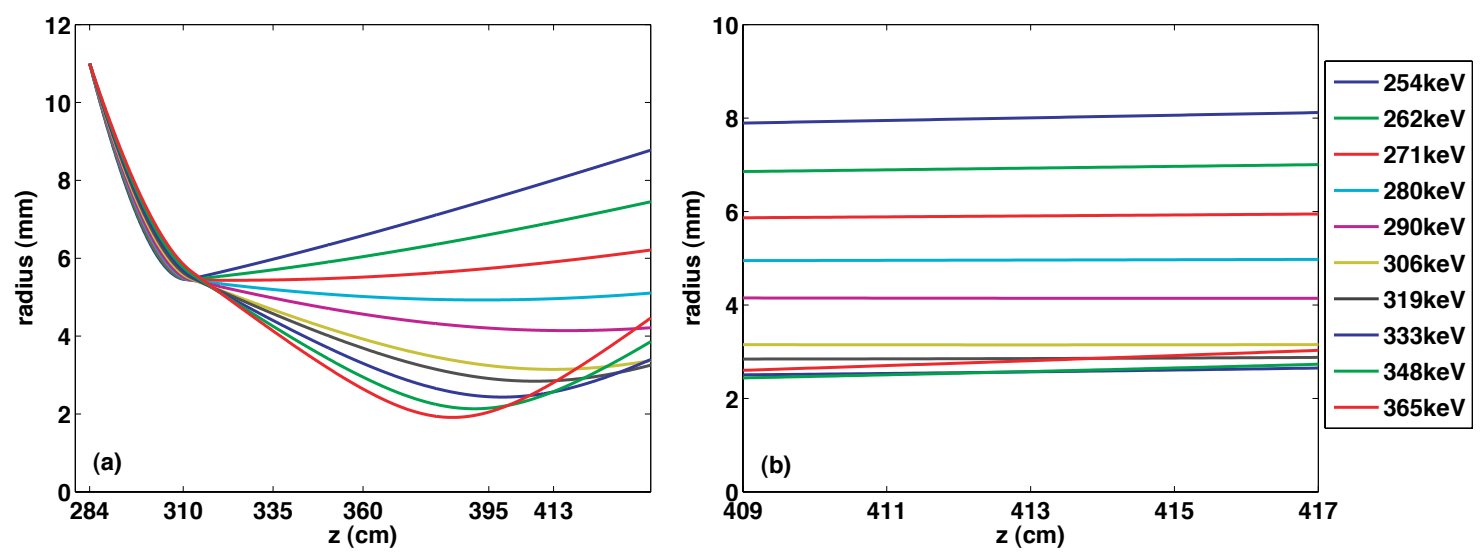

Figure 6.23. Calculated transverse envelopes of different energy slices (a) from the induction gap to the focal plane and; (b) near the focal plane. Energy slices are color coded in legend.

These energy slices are summed up as individual Gaussian slices at the focus to show the individual and total $\varepsilon$. The composite bunch is a peaked distribution composed of broad, lower intensity Gaussian distributions from energy slices with $\mathrm{E}<300 \mathrm{keV}$ and narrow, higher intensity Gaussian distributions from energy slices with E > $300 \mathrm{keV}$ (Fig. 6.24). As stated above, (in Section 6.3) physical aperture limitations on the beam envelope made it difficult to achieve a steeper convergence and higher intensities. The 2rms radius, FWHM, and energy deposition of this composite bunch are: $3.01 \mathrm{~mm}, 3.55 \mathrm{~mm}$, and $9 \mathrm{~mJ} / \mathrm{cm}^{2}$. These calculated results are close to what was measured in Section 6.3.1, however there are slight differences between this calculation, LSP, and the measured energy deposition due to slightly different final spot sizes for each case.

A reduction in the actual plasma density on target versus the ideal case with sufficient plasma in the calculation may help explain the slight differences in intensity between the envelope model and the measurements in Section 6.3.1. The plasma density was measured near the target for this geometry after beam experiments and a decreased plasma density on target is shown in Fig. 6.25 [128]. Comparing this 


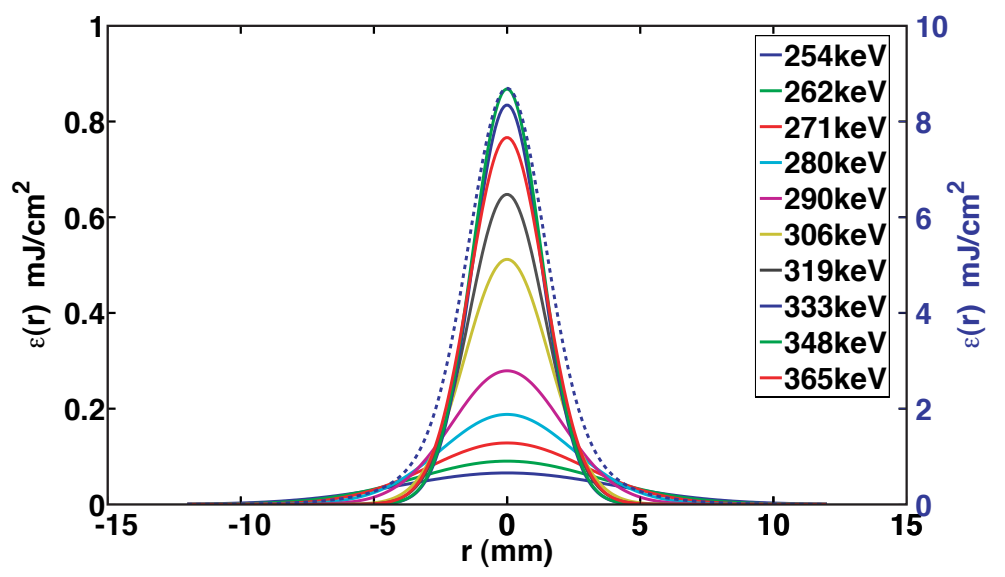

Figure 6.24. Individual Gaussian slices at the focal plane and the composite bunch (dashed blue line). Energy slices are color coded in legend. The left vertical axis is for individual beam bunches and the right vertical axis is for the composite beam bunch.

to the expected beam density for a perfectly neutralized case there is an obvious crossover near the target. This subjects a converging partially neutralized beam to space charge forces resulting in decreased intensity at focus, helping explain the reduction in intensity measured in Section 6.3.1.

\subsubsection{Focusing with a solenoid}

More recent experiments on the NDCX used a final focus solenoid to help increase the intensity on target by providing a steep convergence angle to the beam just upstream of the target. This research is ongoing. The focusing geometry downstream of the IBM is changed slightly from Fig. 6.1. In addition to the dipole magnets added for steering (described in Section 5.6.2), a smaller beam pipe (radius of $19 \mathrm{~mm}$ ) was added downstream of the ferroelectric plasma column. This smaller beam pipe was used to couple the $10-\mathrm{cm}$ long high field solenoid to the target chamber downstream and transport section upstream. 


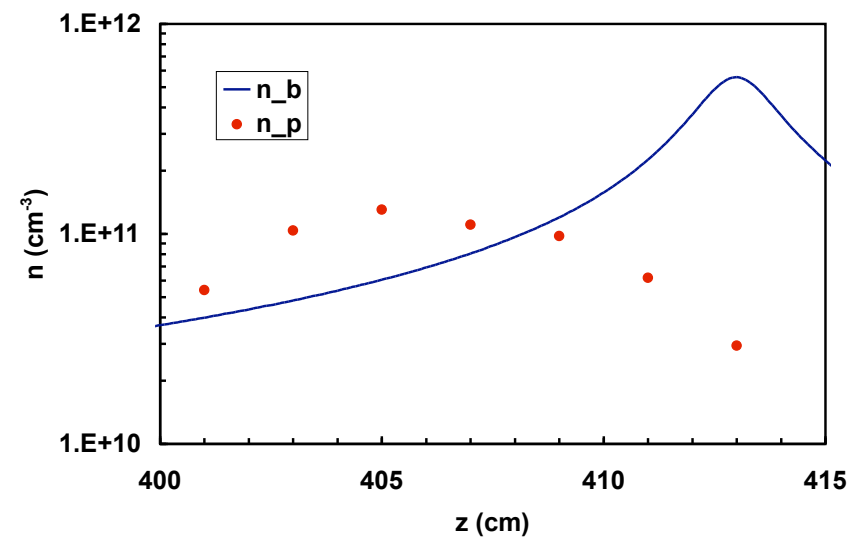

Figure 6.25. Measured plasma density and expected beam density for a perfectly neutralized case both near the target.

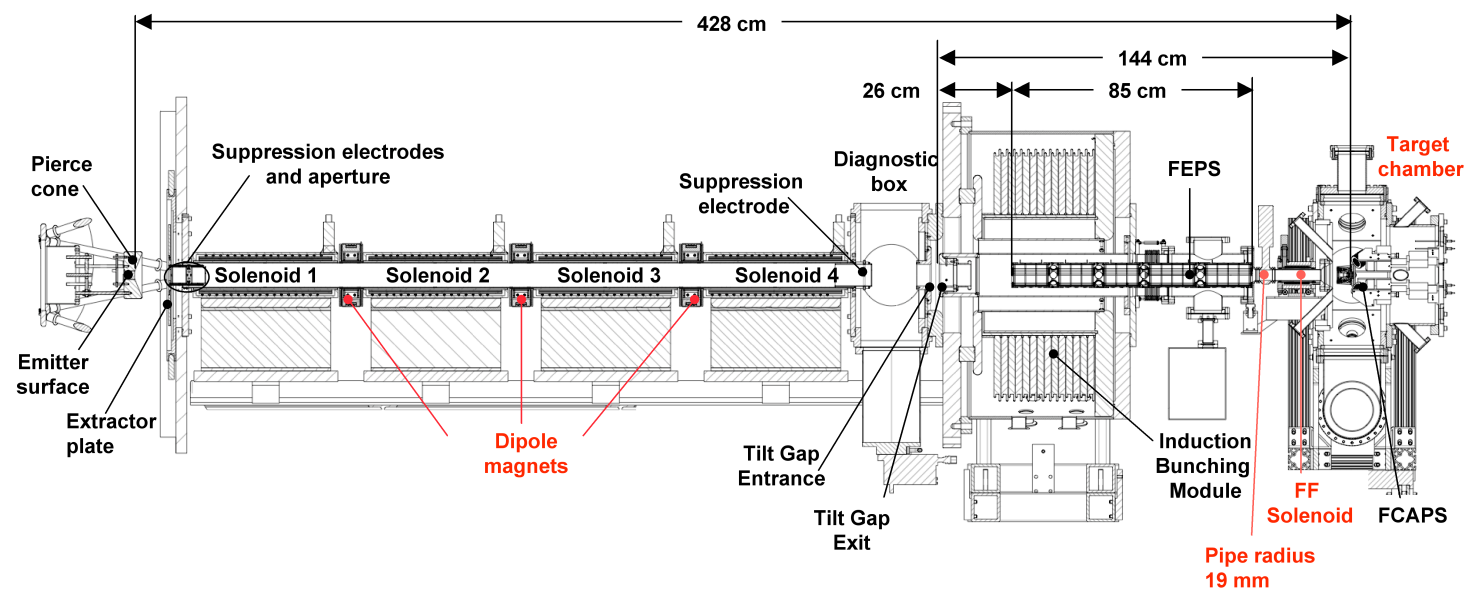

Figure 6.26. Elevation view of the Final Focusing Experiment on NDCX with changes from Fig. 6.1 labeled in red. 

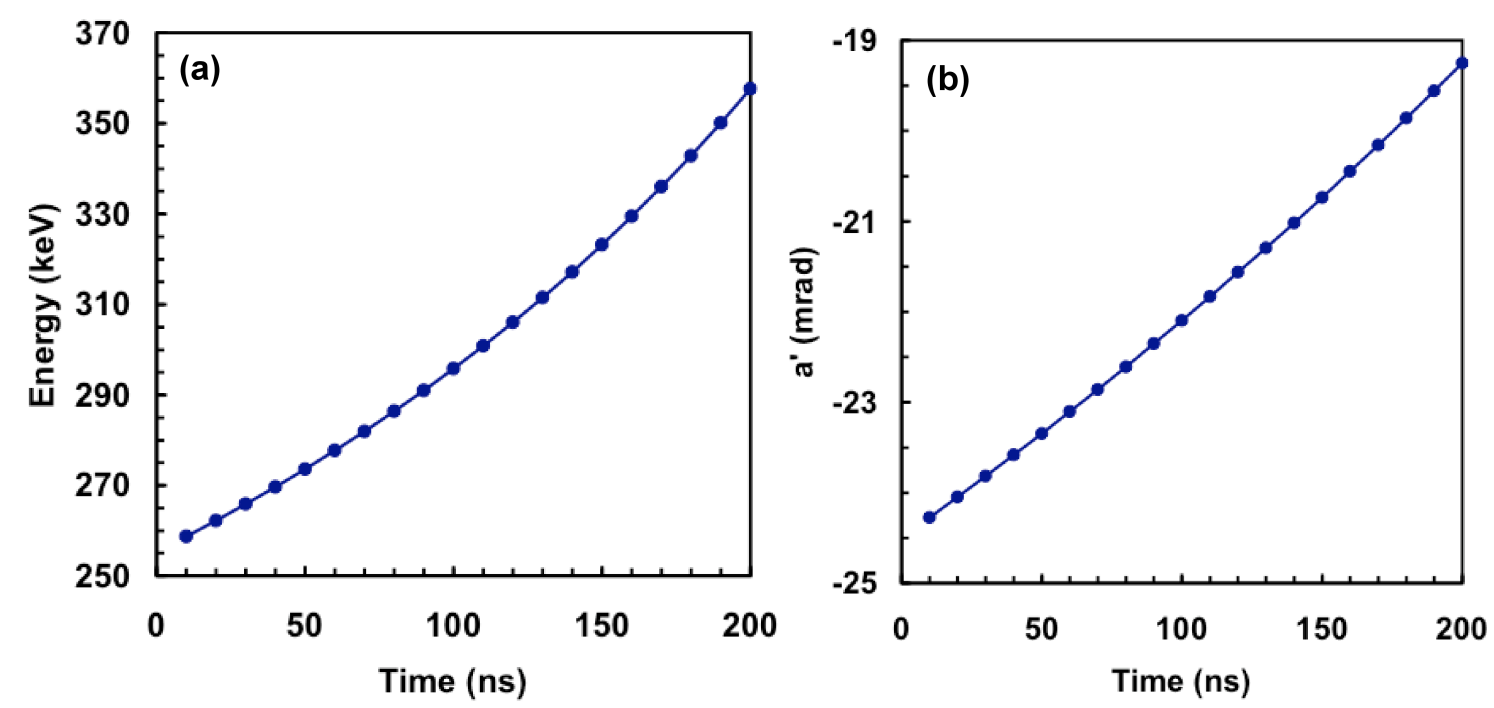

Figure 6.27. Calculated (a) change in axial velocity and; (b) transverse envelope angle of different energy slices.

The beam envelope designed for optimum focus has envelope parameters of $\mathrm{a}=9$ $\mathrm{mm}$ and $\mathrm{a}^{\prime}=-25 \mathrm{mrad}$ at the entrance of the IBM. Due to the defocusing effect the energy slice with no $\Delta \mathrm{v}_{\mathrm{z}}(\mathrm{E}=300 \mathrm{keV})$ had a reduction in the convergence angle to $-22 \mathrm{mrad}$. The effect on the remaining portion of the bunch is shown in Fig. 6.27, the head of the beam bunch $(\mathrm{t}=0 \mathrm{~ns})$ gets decelerated and only has a slight change in envelope angle and the tail of the beam bunch $(\mathrm{t}=200 \mathrm{~ns})$ gets accelerated and has a decrease of almost 6 mrad in the convergence angle.

For this case the energy slice with no $\Delta \mathrm{v}_{\mathrm{z}}$ was designed to reach a space-charge dominated waist before entering the neutralization section at $\mathrm{z}=310 \mathrm{~cm}$ with a diverging angle [Fig. 6.28(a)]. This energy slice diverged to a radius of $11 \mathrm{~mm}$ at the entrance of the final focusing solenoid $(\mathrm{z}=405 \mathrm{~cm})$ before focusing onto the target at $\mathrm{z}=428 \mathrm{~cm}$. The purpose of designing the beam with a diverging envelope was a function of the focal length. The focal length (Eq. 2.57) for $300 \mathrm{keV} \mathrm{K} \mathrm{K}^{+}$ ions with a zero envelope angle through a $10 \mathrm{~cm}, 8 \mathrm{~T}$ solenoid is $15.2 \mathrm{~cm}$ and the minimum distance to focus provided by the hardware was $18 \mathrm{~cm}$. So the beam could 

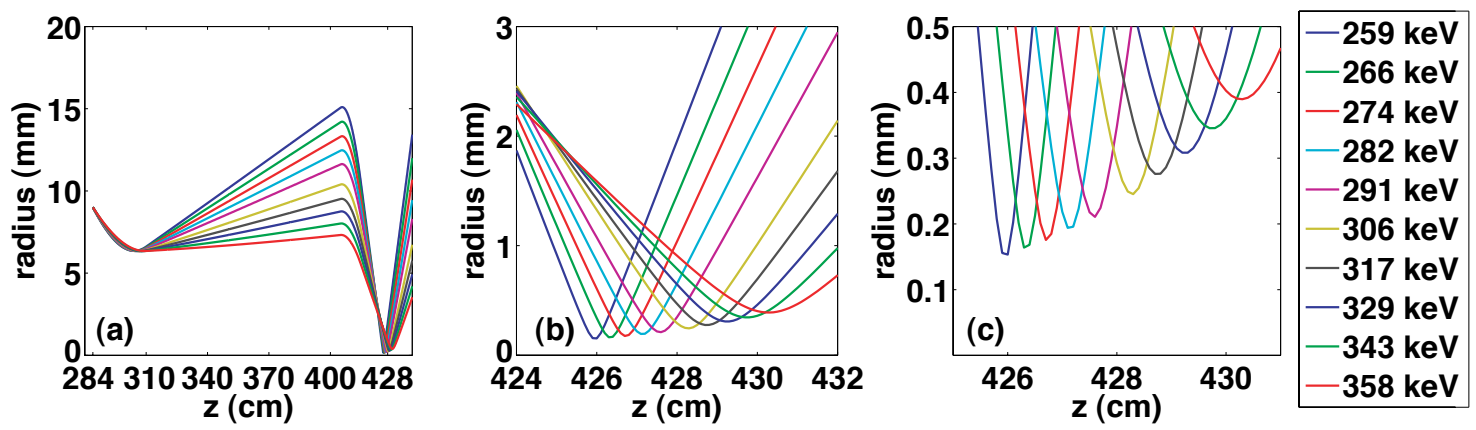

Figure 6.28. Calculated transverse envelopes of different energy slices (a) from the induction gap to the focal plane; (b) near the focal plane and; (c) zoomed in to the see all of the beam waists. Energy slices are color coded in legend.

not enter the solenoid with zero or converging envelope angle or it would come to a focus upstream of the target.

All of the remaining energy slices have such a steep angle at the exit of the IBM $(\mathrm{z}=284 \mathrm{~cm})$ they also come to a space charge dominated waist before entering the neutralization section $(\mathrm{z}=310 \mathrm{~cm})$ [Fig. 6.28(a)]. As expected the lower energy slices come to a waist upstream of the neutralization and the higher energy slices come to a waist at the commencement of neutralization. Like the energy slice with no $\Delta \mathrm{v}_{\mathrm{z}}$ all of the remaining energy slices diverge until entering the final focusing solenoid. The spread in the envelopes in quite large with a $\sigma=2.39 \mathrm{~mm}$. The lower energy slices diverge the most yet they focus upstream (are diverging at the target) and the higher energy slices come to a focus downstream (are converging at the target) [Figs. $6.28(\mathrm{~b}) \&(\mathrm{c})]$.

Again the energy slices are summed up as individual Gaussian slices at the focus. The composite bunch is a peaked distribution composed of broad, lower intensity Gaussian distributions from beam energies with large $\Delta \mathrm{v}_{\mathrm{z}}$ and narrow, higher intensity Gaussian distributions from beam energies with small $\Delta \mathrm{v}_{\mathrm{z}}$ (Fig. 6.29). The 2rms radius, FWHM, and energy deposition of this composite bunch are: $339 \mu \mathrm{m}, 399 \mu \mathrm{m}$, 


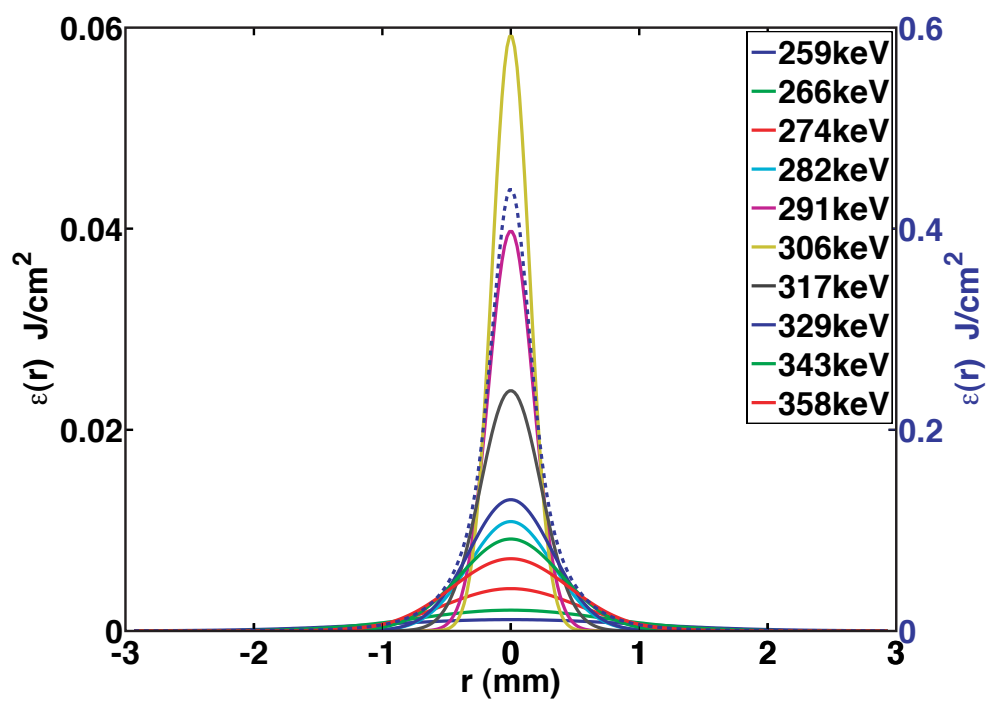

Figure 6.29. Individual Gaussian slices at the focal plane and the composite bunch (dashed blue line). Energy slices are color coded in legend. The left vertical axis is for individual beam bunches and the right vertical axis is for the composite beam bunch.

and $0.439 \mathrm{~J} / \mathrm{cm}^{2}$. The aberration causes a reduction in intensity of 4.16 , however the intensity is still sufficient to provide target heating of thin $(\leq 1 \mu \mathrm{m})$ metallic foils $(\mathrm{Al}$ or $\mathrm{Au})$ to electron temperatures $\sim 0.1 \mathrm{eV}$. It should also be noted that for a slightly different focusing geometry with similar initial conditions we have calculated $0.39 \mathrm{~J} / \mathrm{cm}^{2}$ with this envelope model, which is in qualitative agreement with LSP simulations that have calculated $0.3 \mathrm{~J} / \mathrm{cm}^{2}$.

Again perfect neutralization is assumed in these calculations and plasma density measurements show this is not likely to be the case. The plasma density was measured near the target for this geometry and a decreased plasma density on target is shown in Fig. 6.30 [129]. Comparing this to the expected beam density for a perfectly neutralized case there is an obvious crossover near the target. This will induce a converging neutralized beam to space charge forces and result in decreased intensity at focus. 


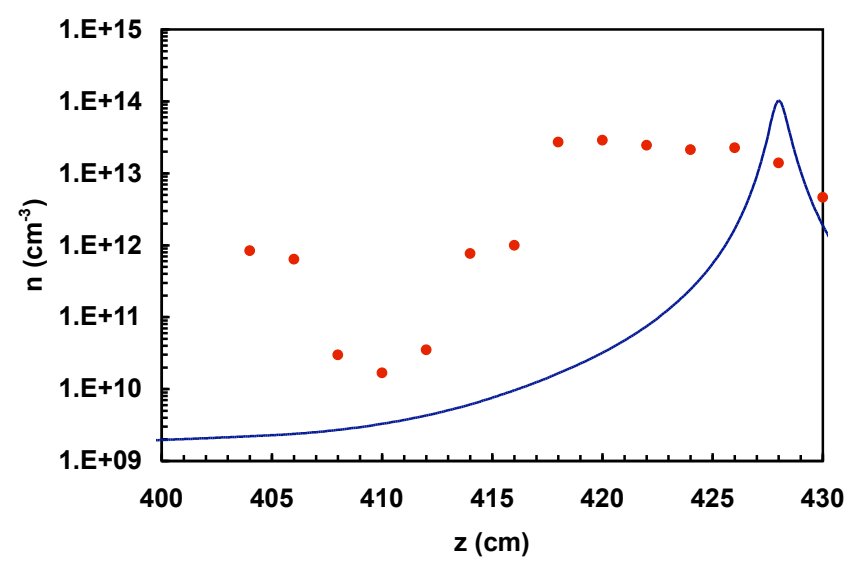

Figure 6.30. Measured plasma density and expected beam density for a perfectly neutralized case both near the target.

\subsection{Plasma neutralization challenges with a high field solenoid}

Experiments and simulations being conducted with the final focus geometry have indicated the difficulty in filling the beam transport section with the necessary amount of plasma to yield perfect neutralization (Fig. 6.30). The final focusing solenoid makes it difficult to fill the region occupied by the beam with plasma. The plasma is strongly coupled to the field lines provided by the final focus solenoid (Fig. 6.31). However, this is disadvantageous because it prevents most of the plasma from crossing field lines and filling in the last few $\mathrm{cm}$ upstream of the target and at the target plane with enough plasma. This last $5 \mathrm{~cm}$ upstream of the target plane is the most critical location for sufficient plasma because it is where the beam density, for a perfectly neutralized case, jumps up from $10^{11} \mathrm{~cm}^{-3}$ to nearly $10^{14} \mathrm{~cm}^{-3}$ (Fig. 6.30).

Another feature of the final focus solenoid is the FCAPS only sample a finite amount of the flux lines provided by the solenoid because they are a finite size and reside in the fringe field of the solenoid (Fig. 6.31). This reduces the effective volume 


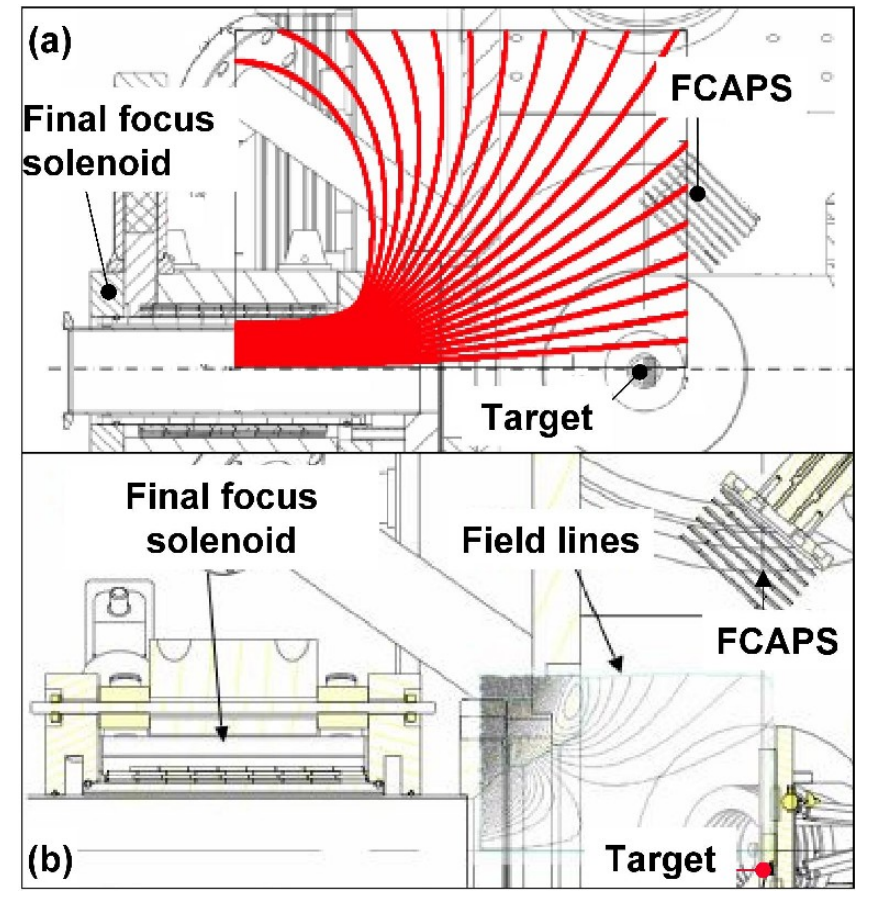

Figure 6.31. Magnetic flux calculations in the final focus geometry (a) with the solenoid only; (b) including eddy current effects from the stainless steel beam pipe and flanges. Note the geometries are slighly different in both cases. 
of plasma that fills the solenoid upstream. This leads to a narrow plasma distribution inside the solenoid, limiting beam neutralization. A possible solution is to either modify the flux lines provided by the solenoid or have plasma sources organized at different locations so that the maximum amount of area can be accessed.

Finally, an additional feature called magnetic mirroring can also limit the flow of plasma into the final focus solenoid. Once the plasma is injected from each of the FCAPS it converges due to magnetization. However, the field continues to increase as you approach the final focus solenoid and the magnetic mirror effect begins to play a role in these larger fields. Particles that approach the increasing field strength with a parallel velocity $\gg$ their perpendicular velocity will be reflected. As a result the final amount of plasma accepted into the solenoid is reduced.

\subsection{Conclusions}

The addition of the FEPS along with slight improvements in the measurement techniques and induction velocity tilt help explain the improved axial focus $(>100$ axial compression, $<2$ ns pulses). We have also successfully demonstrated a nearly three-fold reduction in spot size to demonstrate a simultaneously focused spot with $\mathrm{a}$ and $\mathrm{b}<5 \mathrm{~mm}$. This is consistent with calculations from LSP simulations, though

the beam intensity is not yet what is desired for WDM experiments $\left(\sim 0.1 \mathrm{~J} / \mathrm{cm}^{2}\right)$ $[130]$.

A further reduction in spot size is possible with a high field solenoid upstream of the target. However, low plasma density near the target and plasma injection into a high field solenoid present difficulty for effective neutralization of the beam space charge, resulting in a reduced energy deposition on target. 


\section{Chapter 7}

\section{Longitudinal beam dynamics}

\section{measurements}

\subsection{Experimental layout}

An additional experimental configuration was used to study the limits of axial compression and the longitudinal dynamics of the $0.3-\mathrm{MeV}, 30-\mathrm{mA}$, singly charged $\mathrm{K}^{+}$ion beam used on the NDCX. An electrostatic energy analyzer (EEA) was added at the exit of the FEPS (Fig. 7.1) to measure the longitudinal phase space and temperature of the beam with and without plasma neutralization. The measured longitudinal temperature before axial compression determines an upper limit on axial compression and constrains assumptions in theoretical models of the experiment.

\subsubsection{Lattice and beam envelopes}

The configuration of the focusing lattice used [Fig. 7.2(a)] was designed to project the beam waist as far downstream from the focusing lattice as possible, and as close 


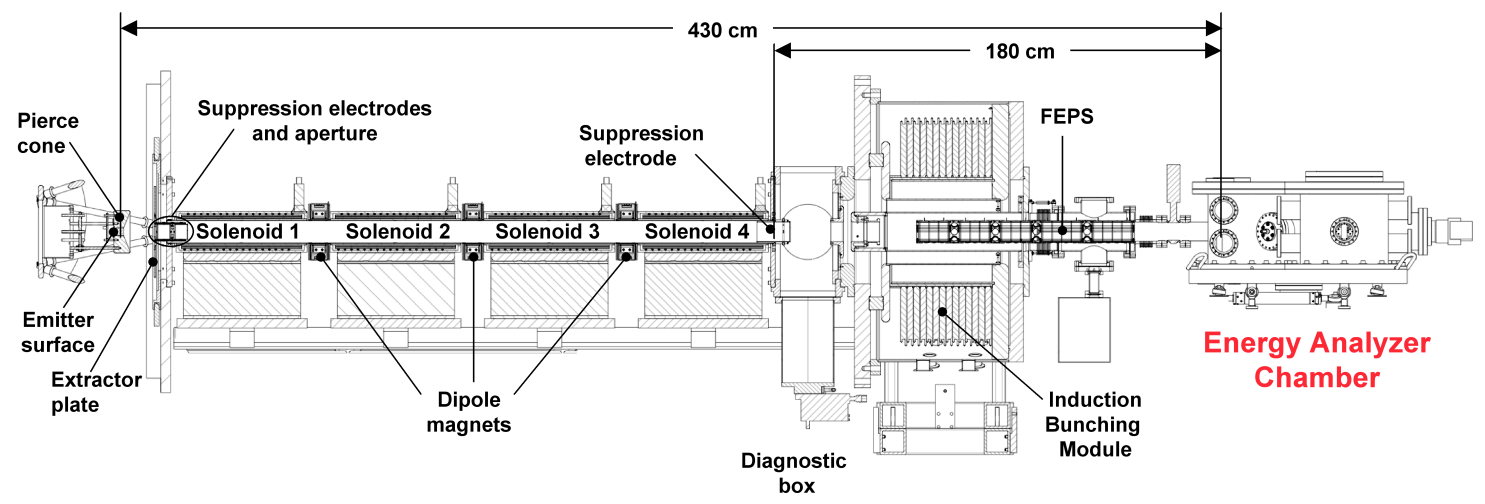

Figure 7.1. Elevation view of the NDCX with the EEA added on at the exit of the FEPS.
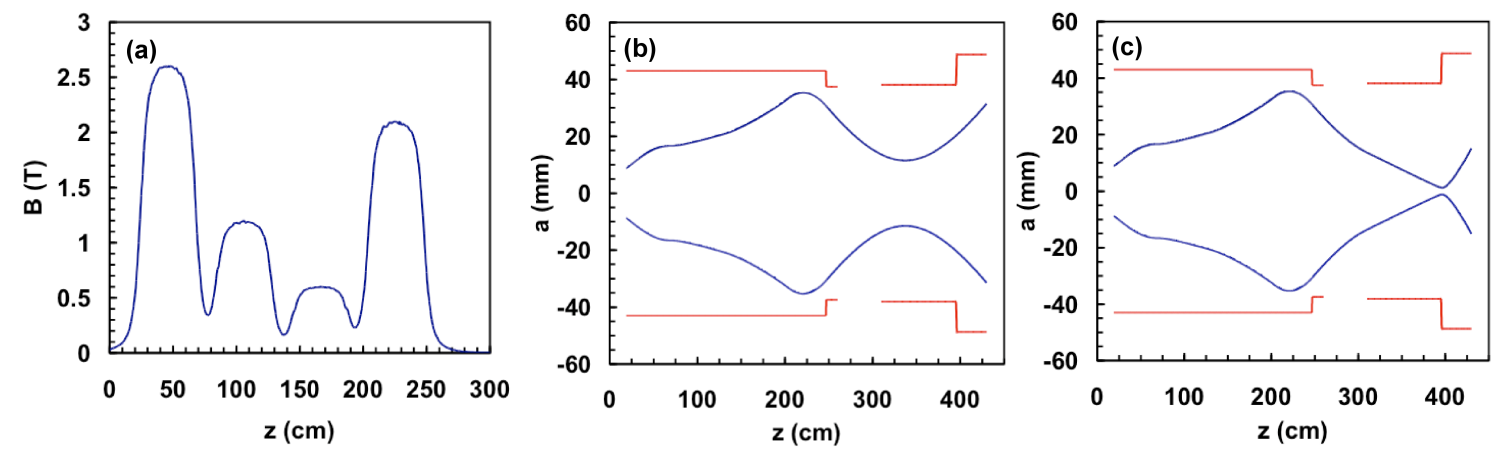

Figure 7.2. (a) Axial magnetic field profile based on measurements; (b) calculated beam envelope for an unneutralized beam and; (c) calculated beam envelope for a beam neutralized by the FEPS.

to the entrance of the EEA as possible. The purpose was to maximize the current density of the beam entering the spectrometer without scraping upstream. However, the current density must not be too high $\left(\mathrm{J}>40 \mathrm{~A} / \mathrm{m}^{2}\right)$ because space charge effects begin to play a role with $\mathrm{I}>25 \mu \mathrm{A}$ in the spectrometer. The resulting beam envelope, shown in Fig. 7.2(b), safely transported the unneutralized beam to the entrance of the spectrometer without scraping and the neutralized case as well [Fig. 7.2(c)].

The beam distribution was measured at the exit of the four-solenoid lattice to quantify if the transverse envelope parameters and centroid offsets were within the 
margin of error to avoid scraping. Slight adjustments were made with the dipoles to correct the centroid offsets to avoid beam scraping.

\subsubsection{Ion trajectories}

Upon entering the spectrometer the beam passed through a $0.1 \mathrm{~mm} \times 5 \mathrm{~mm}$ slit plate (Fig. 7.3). This slit reduced the transmitted beam current from $30 \mathrm{~mA}$, which was incident on the slit plate, to $\sim 10 \mu \mathrm{A}$ for current densities $\sim 20 \mathrm{~A} / \mathrm{m}^{2}$. The beam ribbon traversed the $90^{\circ}$ bend of the electrostatic dipole and was detected at the focal plane. The trajectory of ions of various energies through the spectrometer is illustrated in the schematic in Fig. 7.3. Ions with an energy greater than the corresponding energy on the dipole will follow a larger radius of curvature through the spectrometer $\left(\mathrm{E}_{3}\right)$ and will have a positive first moment on the $\mathrm{x}$-axis at the diagnostc plane $\left(+\left\langle\mathrm{x}_{\mathrm{o}}\right\rangle\right)$, where $\left\langle\mathrm{x}_{\mathrm{o}}\right\rangle=0$ is the center of the diagnostic plane. Ions with an energy less than the dipole will follow a smaller radius of curvature through the spectrometer $\left(\mathrm{E}_{1}\right)$ and will have a negative first moment on the $\mathrm{x}$-axis $\left(-\left\langle\mathrm{x}_{\mathrm{o}}\right\rangle\right)$.

Measurements were made optically at the focal plane with a $100-\mu \mathrm{m}$ thick alumina scintillator, similar to that described in Sections 3.1.3. The beam-induced light emission was captured by an image intensified gated-CCD camera. The transverse resolution was $0.07 \mathrm{~mm} /$ pixel, which is better than the resolution of the spectrometer relative to the slit configuration used $(0.1 \mathrm{~mm})$ for these measurements. Consecutive measurements were made at different times throughout the $3 \mu$ s beam pulse to determine the narrowest transverse distribution or energy profile. It was determined that a $500 \mathrm{~ns}$ gate time delayed to $\mathrm{t}=5.75 \mu$ s captured the narrowest energy profile, where $\mathrm{t}=0$ is the extraction time of beam from the diode. This corresponded to the same 


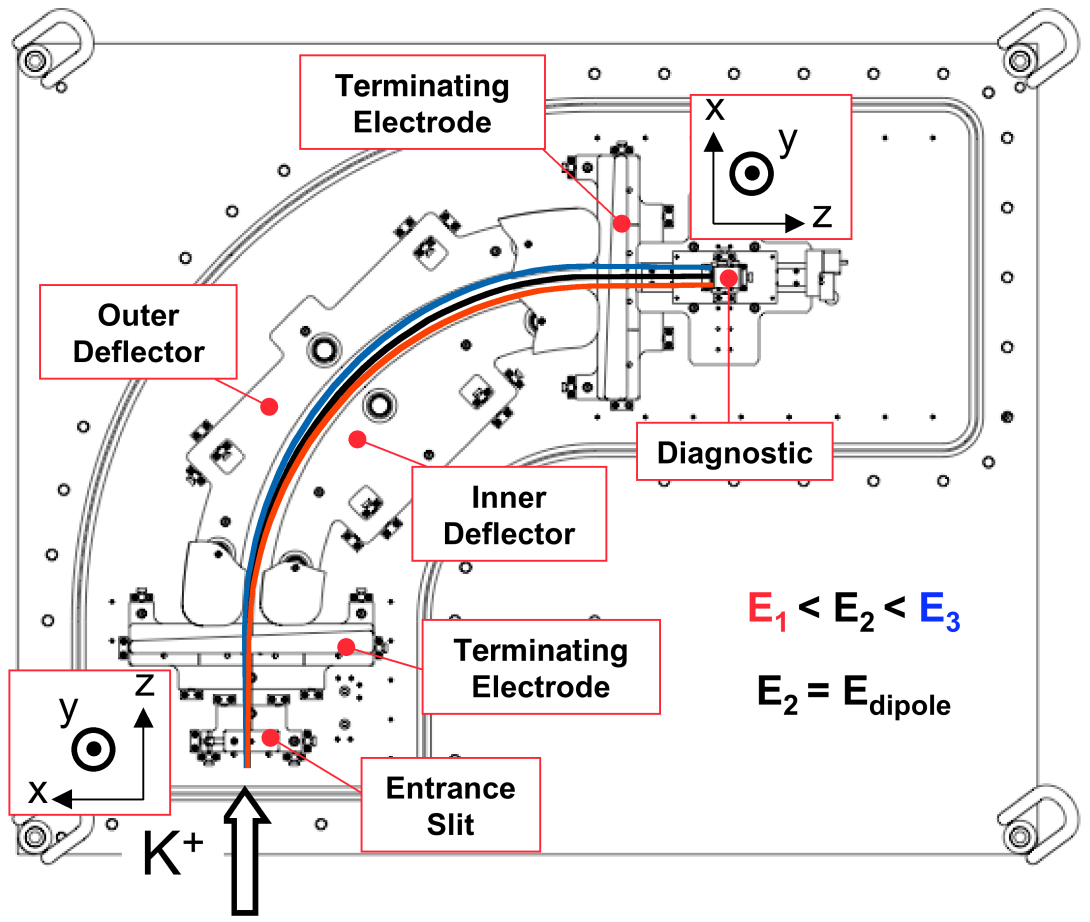

Figure 7.3. Schematic of the ion trajectories though the spectrometer. Ions with an energy greater than the dipole $\left(\mathrm{E}_{3}\right.$, blue) are shown with a larger radius of curvature and ions with an energy less than the dipole $\left(E_{1}\right.$, red) are shown with a smaller radius of curvature. The coordinate system is shown at the entrance and exit of the spectrometer. 


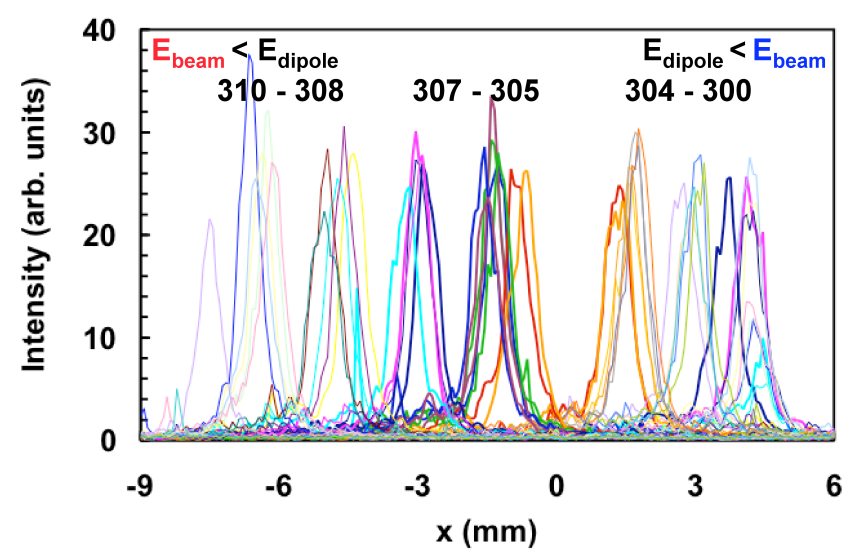

Figure 7.4. Displacement of energy profiles transported through the spectrometer about the focal plane. All profiles are transverse slices of the beam integrated over a $500 \mathrm{~ns}$ gate in the middle of the $3 \mu$ s pulse projected onto the $\mathrm{x}$-axis at the focal plane. The equivalent energy on the dipole is labeled.

time in the middle of the $3 \mu$ s beam pulse where simultaneous focusing measurements were made (Section 6.3.1).

A demonstration of the displacement of the ions at the focal plane is shown in Fig. 7.4. The measurements were taken at the time frame selected above and the dipole field was varied over a range that corresponded to $300 \leq \mathrm{E}_{\text {beam }} \leq 310 \mathrm{keV}$, where $\mathrm{E}_{\mathrm{beam}}$ is the ion kinetic energy along the central trajectory of the spectrometer. The resulting energy profiles were measured with displacements about the x-axis. When $\mathrm{E}_{\text {dipole }}>305 \mathrm{keV}$, where $\mathrm{E}_{\text {dipole }}=20 \mathrm{eV} / \mathrm{V} \cdot \mathrm{V}_{\text {dipole }}$ (Eq. 3.4), the energy profiles typically had a $-\left\langle\mathrm{x}_{\mathrm{o}}\right\rangle$ because the beam energy was lower than the dipole energy. The opposite was the case when $\mathrm{E}_{\text {dipole }}<305 \mathrm{keV}$. The relation of the position of the energy profile to the energy of the beam is given in Eq. (3.10), where a $1 \mathrm{keV}$ change in energy for a $300 \mathrm{keV}$ beam is equivalent to a movement of $1.67 \mathrm{~mm}$ at the focal plane.

The resulting ensemble of distributions were used to determine if there was an effective increase or decrease in the dipole radius from the designed $90^{\circ}$ bend. A 
fiducial on the scintillator was used for reference to the ideal centerline. After relating each of the measured distributions to the fiducial on the scintillator and taking a first moment of the ensemble of distributions it was determined $\left\langle\mathrm{x}_{\mathrm{o}}\right\rangle=-1.65 \mathrm{~mm}$ as shown in Fig. 7.4. This offset translated to an additional $9.4 \mathrm{mrad}\left(0.54^{\circ}\right)$ angle added to the designed $90^{\circ}$ bend. This correction was applied when determining the energy of the bunch for optical measurements.

From this data one can also see there is a variation in position of the energy profile for an individual dipole setting. For all of the dipole settings in this ensemble of distributions, the energy of the beam at this particular time slice (500 ns gate in the middle of the $3 \mu$ s beam pulse) varied $\pm 0.24 \mathrm{keV}$.

\subsection{Erratum}

Several months after data acquisition and analysis of the data presented in Fig. 7.4 and below in Figs. 7.6-7.10, it was determined there was a defect in the charging circuit for one of the plates in the dipole. This defect caused a higher voltage drop than expected and required a higher charge voltage $(\sim 800 \mathrm{~V})$ to effectively transport ions through the spectrometer. A complimenting set of data to Fig. 7.4 was acquired (Fig. 7.5) in order to quantify the difference in absolute energy and make a systematic shift to the data presented in Figs. 7.6-7.10.

This does not have a substantial effect on the measured longitudinal temperature presented below, but analysis of Figs. $7.4 \& 7.5$ indicate a reduction in the initially measured energy of $\sim 16 \mathrm{keV}(\sim 5 \%)$. 


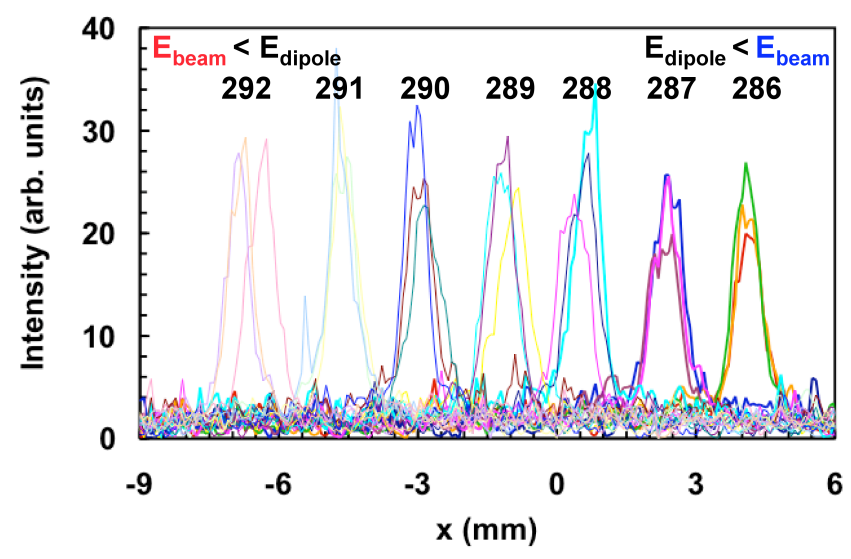

Figure 7.5. Displacement of energy profiles transported through the spectrometer about the focal plane. All profiles are transverse slices of the beam integrated over a 500 ns gate in the middle of the $3 \mu$ s pulse projected onto the $\mathrm{x}$-axis at the focal plane. The corrected equivalent energy on the dipole is labeled.

\subsection{Longitudinal temperature measurements}

The distributions from the data acquired above were used to measure the longitudinal temperature. As explained in Section 3.3.1 (Eq. 3.10) the width $\Delta \mathrm{x}$ of the transverse distribution measured at the diagnostic plane is a function of the geometry of the spectrometer and the energy spread $\Delta \mathrm{E}$ of the beam. Assuming the longitudinal distribution is a 1-D Maxwellian, $\Delta \mathrm{E}$ can be used to calculate the longitudinal temperature, $\mathrm{T}_{\mathrm{z}}$ as explained in Section 2.4.1 (Eq. 2.66)

Measurements were made of the $\mathrm{K}^{+}$ion beam itself and with the space-charge neutralized upstream of the spectrometer by the FEPS. Measurements of the $\mathrm{K}^{+}$ion beam itself displayed a $\mathrm{T}_{\mathrm{z}}=2.4 \pm 1.6 \times 10^{-2} \mathrm{eV}$. Measurements of the neutralized beam showed a slightly warmer temperature, $\mathrm{T}_{\mathrm{z}}=4.7 \pm 2.8 \times 10^{-2} \mathrm{eV}$. The beam distribution of the neutralized case has a current density four times greater than the unneutralized case upon entrance to the spectrometer. This may induce a slight space charge effect, however each of the measurements were within the error bars of 

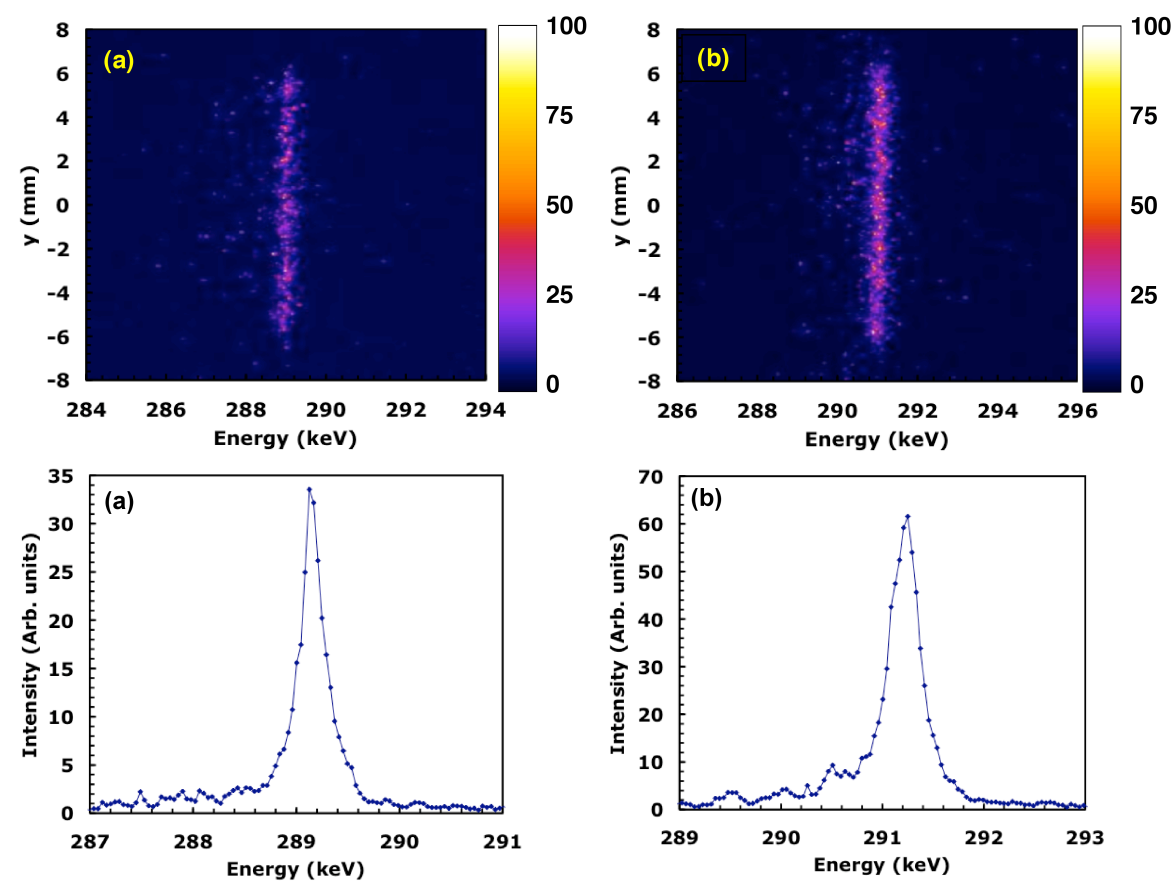

Figure 7.6. Top row: Transverse slices of the beam integrated over a 500 ns gate in the middle of a $3 \mu$ s pulse. Bottom row: Intensity of the transverse slices projected onto the energy axis for: (a) an unneutralized beam and; (b) a beam neutralized by the FEPS (note scale differences).

one another. Two examples of the transverse distributions measured and integrated profiles are shown in Fig. 7.6. It is worth noting that an integrated source to target simulation in LSP [104] calculated $\mathrm{T}_{\mathrm{z}}=2.0 \times 10^{-2} \mathrm{eV}$ for the axial compression yielded in Fig. 6.21.

Measurements were made with narrower time gates (time slices) to verify that the longitudinal temperatures was not influenced over longer gate times. Longitudinal phase space measurements in the next section also verify the time slice measured (middle of the $3 \mu$ s beam pulse) is monochromatic and has a consistent $T_{z}$. 


\subsection{Longitudinal phase space measurements}

\subsubsection{Optical measurements}

The transverse beam distributions from optical measurements with the scintillator were measured as a function of time with an image intensified streak camera (Section 3.3.2). The transverse resolution of the measurements on the CCD was nearly identical to the optical measurements discussed above $(0.072 \mathrm{~mm} / \mathrm{pix})$.

We have taken measurements with the slit of the streak camera set to integrate the beam over 250 ns slices throughout the $3 \mu$ s beam pulse. This cuts down the amount of light collected from previous measurements with the image intensified gated-CCD camera by a factor of four. This is because we are integrating over half as long of a time window and the slit is only capturing $4 \mathrm{~mm}$ of the $10-\mathrm{mm}$ high beam distribution (Fig. 7.6). An average longitudinal phase space distribution from 10 streaked images with a dipole setting of $289 \mathrm{keV}$ is shown in Fig. 7.7. The beam energy fluctuations $(\langle\Delta \mathrm{E}\rangle=240 \mathrm{eV})$ from shot to shot can induce a false widening to the width of the intrinsic distribution.

A sloped region near the head and at the tail of the beam is observed (Fig. 7.7). The slope at the head of the beam is $\sim 4 \mathrm{kV} / \mu$ s from $5.25-5.75 \mu \mathrm{s}$. There is a flat region where the beam energy was relatively constant from 5.75 to $>6.25 \mu \mathrm{s}$. This is also the relevant time frame at which the longitudinal temperature (presented in Section 7.3) and simultaneous focusing measurements (presented in Section 6.3.1) were made, confirming this is the best operating point for axial compression for this

pulse length. After the monochromatic region in phase space the beam energy has a negative slope $\sim 2 \mathrm{kV} / \mu$ s from $\mathrm{t} \geq 6.25 \mu \mathrm{s}$. 


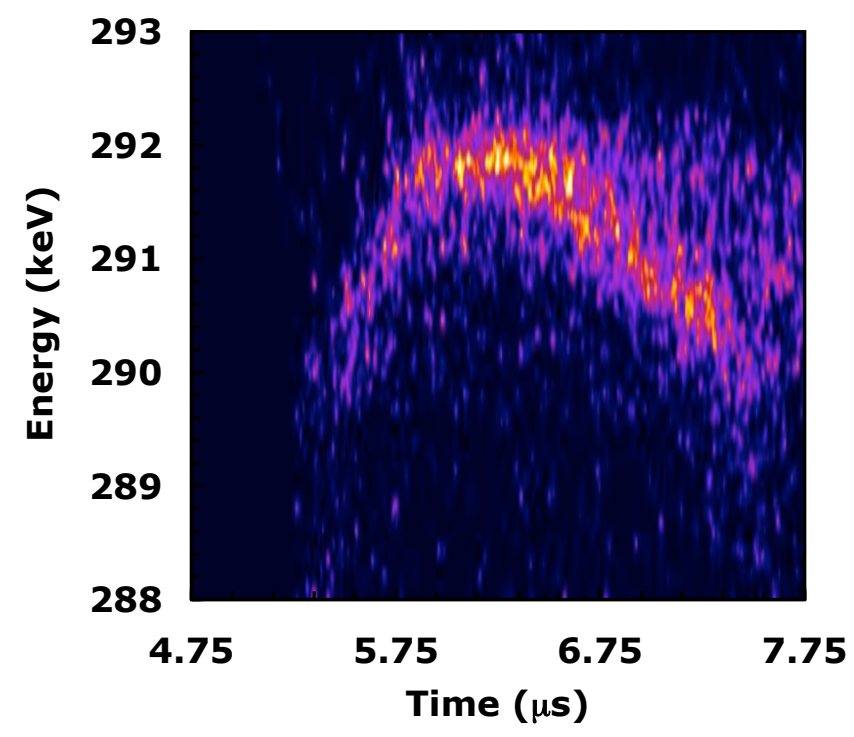

Figure 7.7. Longitudinal phase space distribution of the NDCX beam with a $3 \mu \mathrm{s}$ pulse streaked in time.

\subsubsection{Slit and Faraday collector measurements}

Another method of measuring the longitudinal phase space was made using a slit and a Faraday collector (slit-cup) at the focal plane of the spectrometer. This method is similar to that used for transverse phase space measurements. The slit at the entrance of the spectrometer is the same as described above $(0.1 \mathrm{~mm} \times 5 \mathrm{~mm})$ and the downstream slit was increased to $0.1 \mathrm{~mm} \times 10 \mathrm{~mm}$ to compensate for beam expansion in the vertical (non-bend) plane.

Step sizes for the dipole in these measurements could be as small as the resolution of the spectrometer $(60 \mathrm{eV})$ but larger steps $(200 \mathrm{eV})$ were taken due minimum energy variation of the beam from shot to shot $(\langle\Delta \mathrm{E}\rangle=240 \mathrm{eV})$. The measured distribution for a $2 \mu \mathrm{s}$ beam pulse using this technique is shown in Fig. 7.8. The pulse length for these measurements was shorted to eliminate electrical noise in the circuit due to the Marx crowbar. The $\mathrm{S} / \mathrm{N}$ was already close to 10 when configured to measure 


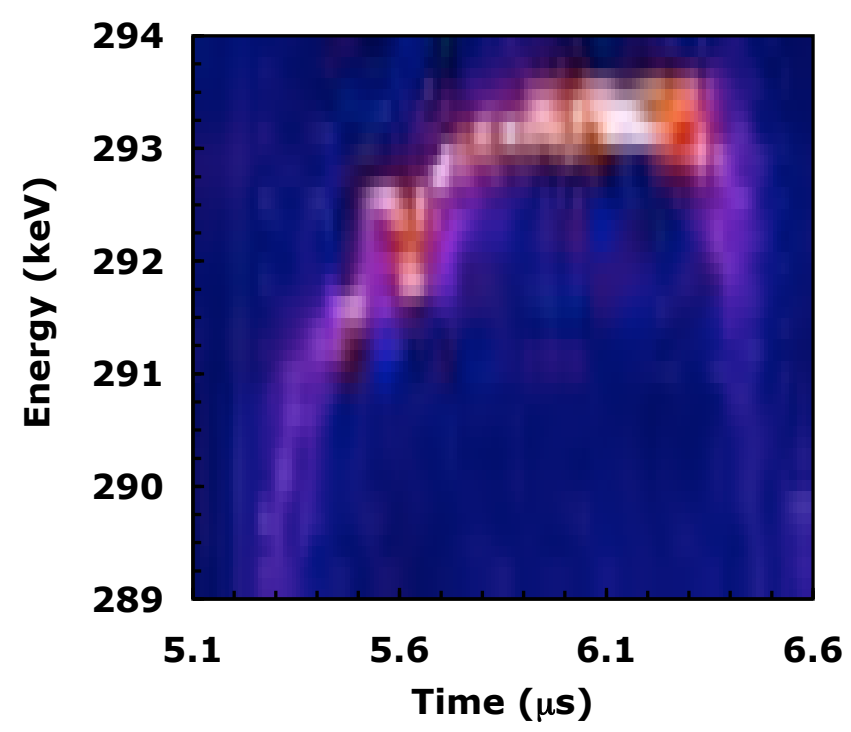

Figure 7.8. Longitudinal phase space distribution of the NDCX beam with a $2 \mu \mathrm{s}$ pulse measured with two slits.

secondary electrons leaving the collector and using an amplifier so any additional noise (i.e. from the crowbar) made the signals difficult to resolve.

An identical slope of $4 \mathrm{kV} / \mu$ s is measured at the head of the beam for this setup when compared to the optically measured longitudinal phase space distribution (Figs. $7.7 \& 7.8)$. There is also a sharp structure at the head of the beam near $5.6 \mu$ s. This could be real or may be an intrinsic effect due to the variation of the beam energy from shot to shot $(\langle\Delta \mathrm{E}\rangle=240 \mathrm{eV})$. Afterwards there is also a monochromatic region where the beam energy was relatively constant for about $500 \mathrm{~ns}$, which was also seen in the optical measurements. 


\subsection{Correlation of the longitudinal measurements with Marx voltage}

Each of the methods of longitudinal measurement discussed in this chapter were compared to the frequently measured Marx voltage waveform, which is used to extract the beam from the diode.

First a detailed comparison was made with the optical measurements of the energy profiles measured in Section 7.1.2 (Fig. 7.9). The time used for the optical measurements of the energy profiles corresponded to about $1.25 \mu$ s after the Marx was pulsed. A strong correlation is shown in the shot to shot variation of the Marx and the variation in the measured beam energy at this time. Although the actual values do not match up exactly the general trend is the same. Each time the voltage varies in the Marx a corresponding change in the beam energy is measured. The average Marx voltage for this particular data set was $304.10 \pm 1.78 \mathrm{kV}$ and the beam energy was $290.26 \pm 1.03 \mathrm{keV}$, each varied $<1 \%$. This energy variation is a factor of five greater than what was determined for a larger data set in Section 7.1.2 for unknown reasons.

It is worth noting from this comparison that the beam energy measured by the EEA is $4.55 \pm 0.57 \%$ lower than the voltage monitored by the Marx capacitive divider. This complements separate measurements that were made in comparison to the Marx capacitive divider in 2004 on the NTX experiment [131]. These measurements were made with a resistive divider and time of flight (TOF) technique. Each displayed a slightly lower trend in energy than monitored by the capacitive divider voltage. The resistive divider voltage was $1.73 \pm 0.56 \%$ less than the capacitive divider voltage and the energy calculated by TOF was $4.28 \pm 0.94 \%$ less than the capacitive divider voltage. 


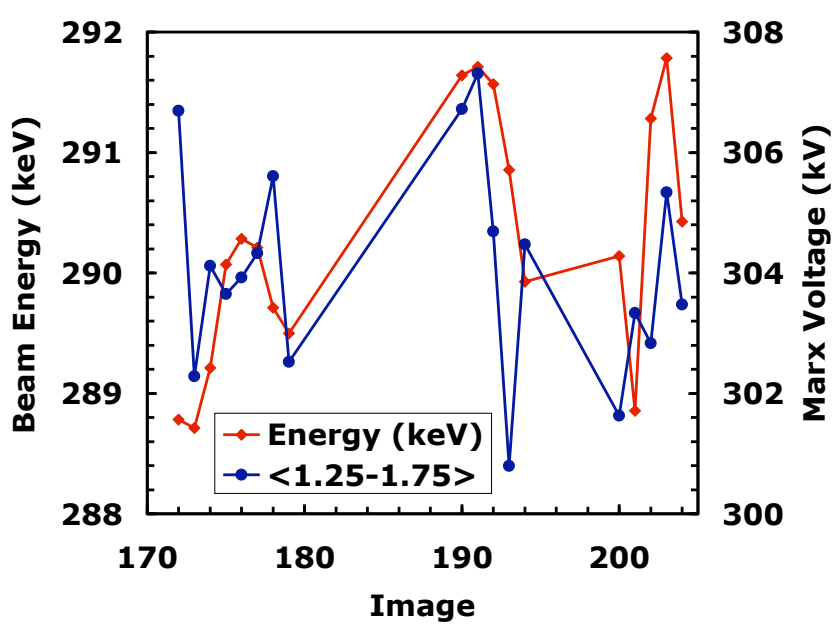

Figure 7.9. Correlation of the Marx voltage with individual measurements of the energy profile made with the CCD camera (note scale differences).

Next the first moments of the longitudinal phase space distributions $(\langle\mathrm{E}\rangle)$ from Figs. $7.7 \& 7.8$ were compared to the average Marx waveforms for the series of shots taken to obtain the distributions. After examining Fig. 7.10 you can see the differences between the Marx waveform and $\langle\mathrm{E}\rangle$; in both cases where there is a steep rise at the beginning of the Marx pulse $(\sim 20 \mathrm{kV} / \mu \mathrm{s})$ and a steady slope down of $4 \mathrm{kV} / \mu$ s after the peak; for the $3 \mu$ s pulse this continues even longer. This is in disagreement with the $\langle E\rangle$ measured in both cases. As described in Section 7.4 the head of the beam in the longitudinal phase space distributions has a slope of $4 \mathrm{kV} / \mu \mathrm{s}$ for $\sim 500 \mathrm{~ns}$, then there is a monochromatic region for $500 \mathrm{~ns}$ or greater. For the $3 \mu \mathrm{s}$ pulse there is an average negative slope of $2 \mathrm{kV} / \mu$ s after the monochromatic region. These results indicate there is a slight sag in the capacitive divider response for Marx waveform. It is also worth noting the average Marx voltage is nearly $2 \mathrm{kV}$ lower for the optical measurements made and the measured beam energy is slightly lower for the optical measurements confirming the errors in the measurement relative to Marx voltage noted above. 

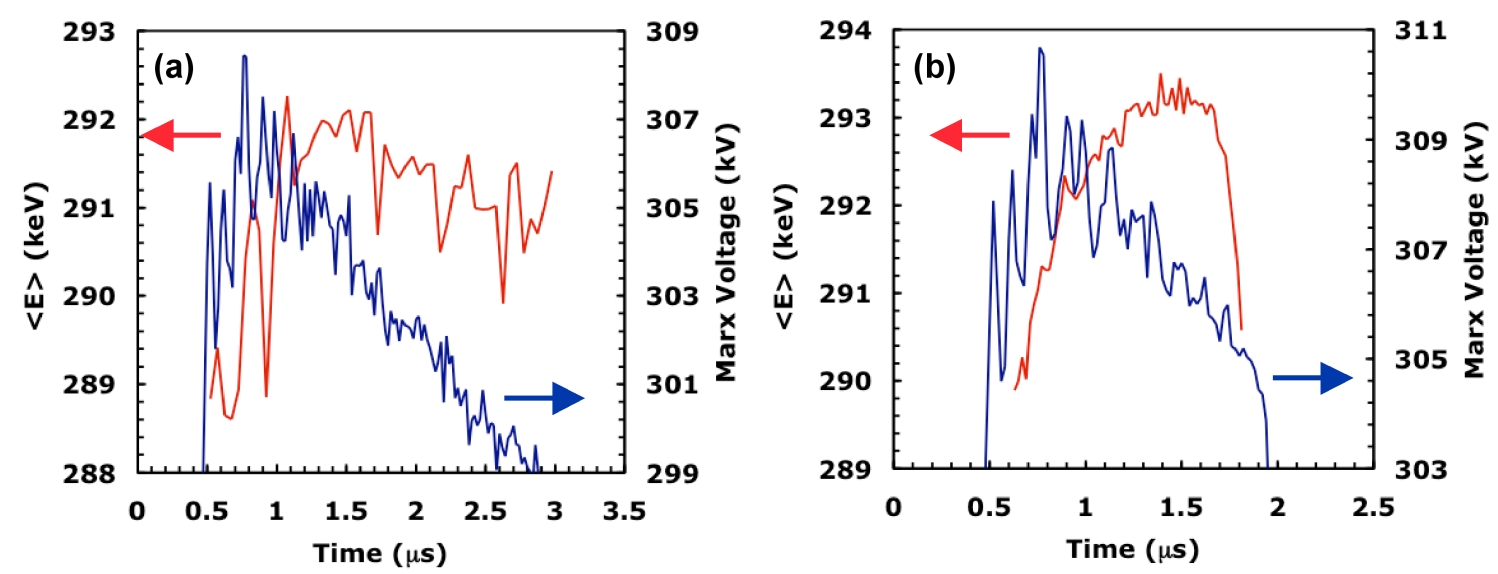

Figure 7.10. Correlation of the Marx voltage (blue) with the $\langle\mathrm{E}\rangle$ (red) resolved from longitudinal phase space measurements with: (a) the streak camera for a $3 \mu$ s pulse and; (b) the slit-cup for a $2 \mu$ s pulse (note scale differences).

Numerical simulations were also performed in Warp and LSP to model the longitudinal dynamics. Both PIC methods used the experimental Marx waveform as an input to calculate the beam dynamics. The calculated distributions show a longitudinal space charge effect near the head of the beam and a distribution that agrees best with the Marx waveform after the head (Fig. 7.11). Both calculations show a $16 \mathrm{kV}$ drop over $100 \mathrm{~ns}$ for the first particles at the head of the distribution. Then a $22 \mathrm{kV}$ increase in energy over $300 \mathrm{~ns}(\sim 73 \mathrm{kV} / \mu \mathrm{s})$ follows. This energy variation due to space charge was not resolved in the longitudinal phase space measurements, which only show a $2 \mathrm{kV}$ rise over $500 \mathrm{~ns}$ at the head. This could possibly be explained by the difference indicated above (Fig. 7.10), that the Marx waveform does not exactly represent the dynamics of the longitudinal beam bunch from head to tail. In addition the current level, or number of particles, at the most upstream portion of the head of the beam is slightly lower than throughout the rest of the beam bunch, which would make these particles difficult to detect. 

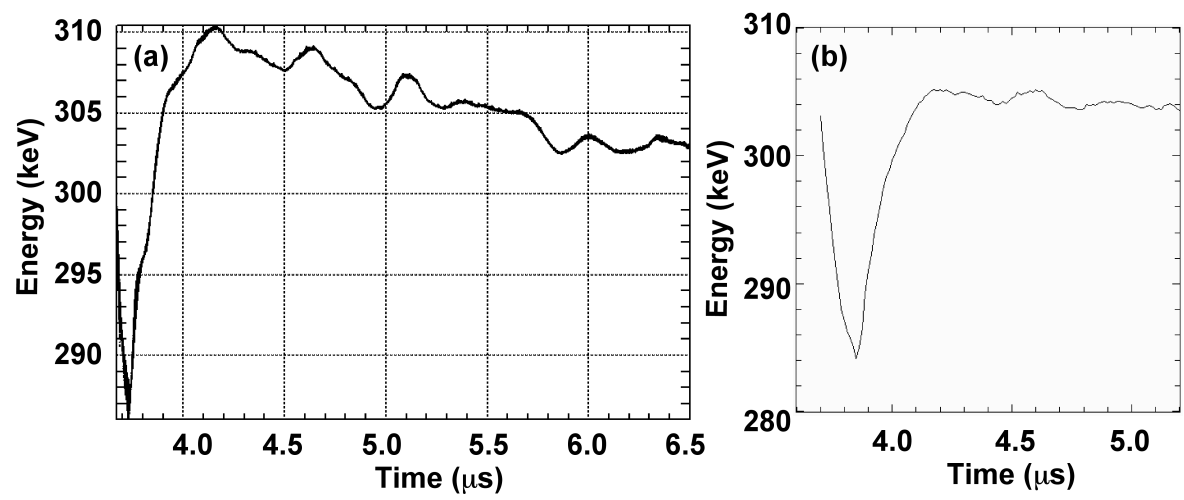

Figure 7.11. Longitudinal phase space distributions calculated in: (a) LSP and; (b) Warp.

\subsection{Conclusions}

We effectively measured the longitudinal temperature and phase space of an intense beam. Measurements of the longitudinal temperature during the relevant time of axial compression indicate $\mathrm{T}_{\mathrm{z}}=2.4 \pm 1.6 \times 10^{-2} \mathrm{eV}$ for the $\mathrm{K}^{+}$ion beam itself and $\mathrm{T}_{\mathrm{z}}=4.7 \pm 2.8 \times 10^{-2} \mathrm{eV}$ for the $\mathrm{K}^{+}$ion beam neutralized by the FEPS. Recent source to target simulations in LSP calculate $\mathrm{T}_{\mathrm{z}}=2 \times 10^{-2} \mathrm{eV}$ in agreement with the experiment, contrary to the previous assumptions for simulations. These measurements set an upper bound on the minimum longitudinal temperature achievable on NDCX in addition to an upper bound on the minimum pulse widths achievable for axial compression. This provides additional support to improve the temporal resolution of the fast diagnostics currently used to measure axial compression (i.e. the fast pinhole Faraday cup and scintillator material).

The Marx voltage waveform appears to provide a qualitative measurement of the beam energy, although comparisons indicate the beam energy measured by the EEA is $4.55 \pm 0.57 \%$ lower than the voltage monitored by the Marx capacitive divider. This is in agreement with past measurements. Longitudinal phase space measurements 
show a slight difference between the longitudinal dynamics and the Marx waveform. Calculations in Warp and LSP show a space charge effect at the beam head that was not measured. The calculations also reproduce the slopes measured in the Marx waveform giving slight disagreement between the calculations and measurements of the longitudinal phase space. This indicates the Marx waveform does not accurately represent the dynamics of the longitudinal beam bunch from head to tail. 


\section{Chapter 8}

\section{Conclusions}

\subsection{Experimental achievements}

The NDCX has demonstrated capabilities for initial target heating experiments. Listed below are a series of experimental achievements separated by chapter.

\subsubsection{Chapter 5}

In Chapter 5 we successfully demonstrated matching and transport of a spacecharge dominated ion beam in a two-solenoid lattice with little or no emittance growth. It is evident from the results presented in Section 5.3 that large fringe magnetic fields are responsible for electron confinement and contribute to the sheath formation and electron cloud effects observed, confusing measurements with intercepting diagnostics. Moving the beam intercepting diagnostics into a nearly magnetic field-free region provided the correct measurement of the beam dynamics and the emittance.

Precise placement of the emitter and alignment of the axial magnetic field in a 
solenoid lattice is critical to the beam dynamics. Evidence of this importance is seen in the measured and calculated beam distributions which are not uniform or axisymmetric, have a centroid offset of several millimeters and milliradians, a hollow center, and a substantial halo. Although these undesired effects have little impact on the emittance in the two solenoid experiment, they may grow in longer focusing lattices and contribute to emittance growth or beam loss.

Electron cloud studies were successfully conducted in a four-solenoid lattice using cylindrical electrodes that intercepted the expanding magnetic flux from the solenoids. Beam dynamics measurements and measurements from the electrodes confirmed that the current reducing aperture used in the experiments provided an $n_{e} \sim 0.01-0.06$ $\mathrm{n}_{\mathrm{b}}$, enough to partially neutralize the beam and cause the emittance to grow $\geq$ 40\%. Beam dynamics measurements proved that using the electrodes to clear these electrons was effective, prevented partial neutralization, and reduced the measured emittance so that it was conserved throughout the lattice.

The dynamics of beam-induced gas desorption, ionization, and electron emission for normal incidence were characterized for a $10 \mu \mathrm{s}$, singly charged $\mathrm{K}^{+}$ion bunch at an ion energy of $0.3 \mathrm{MeV}$ and current of $26 \mathrm{~mA}$. This beam provided $1.6 \times 10^{12}$ ions/pulse at a repetition rate of $0.05 \mathrm{~Hz}$. These measurements showed the gas cloud continues to expand as a function of time and the dynamics are dependent upon the incident material and the bias voltage. For a single pulse the gas desorbed and ionized reached $87 \%$ of the total $\mathrm{K}^{+}$ion current for the stainless steel target by the end of the pulse and $98 \%$ for the copper target. The measured secondary emission coefficient for the stainless steel target was 10.87 and was 9.18 for copper. For each of the targets the total ionized gas and electron densities approached the beam density.

A successful correction of the beam centroid has been demonstrated with dipole magnets using a Jacobian based method. An analytic method was developed to 
examine the offsets of individual solenoids and quantify their individual and total contributions to the centroid motion. This has the potential to simplify the correction process and provide a better understanding of the expected centroid motion for different lattice tunes.

\subsubsection{Chapter 6}

In Chapter 6 the addition of the FEPS along with slight improvements in the measurement techniques and induction velocity tilt help explain the improved axial focus ( $>100$ axial compression, $<2$ ns pulses). We have also successfully demonstrated a nearly three-fold reduction in spot size to demonstrate a simultaneously focused spot with a and $\mathrm{b}<5 \mathrm{~mm}$. This is consistent with calculations from LSP simulations, though the beam intensity is not yet what is desired for WDM experiments $(\sim 0.1$ $\left.\mathrm{J} / \mathrm{cm}^{2}\right)[130]$.

A further reduction in spot size is possible with a high field solenoid upstream of the target. However, low plasma density near the target and plasma injection into a high field solenoid present difficulty for effective neutralization of the beam space charge, resulting in a reduced energy deposition on target.

\subsubsection{Chapter 7}

In Chapter 7 we effectively measured the longitudinal temperature and phase space of an intense beam. Measurements of the longitudinal temperature during the relevant time of axial compression indicate $\mathrm{T}_{\mathrm{z}}=2.4 \pm 1.6 \times 10^{-2} \mathrm{eV}$ for the $\mathrm{K}^{+}$ ion beam itself and $\mathrm{T}_{\mathrm{z}}=4.7 \pm 2.8 \times 10^{-2} \mathrm{eV}$ for the $\mathrm{K}^{+}$ion beam neutralized by the FEPS. Recent source to target simulations in LSP calculate $\mathrm{T}_{\mathrm{z}}=2 \times 10^{-2} \mathrm{eV}$ in agreement with the experiment, contrary to the previous assumptions for simulations. 
These measurements set an upper bound on the minimum longitudinal temperature achievable on NDCX in addition to an upper bound on the minimum pulse widths achievable for axial compression. This provides additional support to improve the temporal resolution of the fast diagnostics currently used to measure axial compression (i.e. the fast pinhole Faraday cup and scintillator material).

The Marx voltage waveform appears to provide a qualitative measurement of the beam energy, although comparisons indicate the beam energy measured by the EEA is $4.55 \pm 0.57 \%$ lower than the voltage monitored by the Marx capacitive divider. This is in agreement with past measurements. Longitudinal phase space measurements show a slight difference between the longitudinal dynamics and the Marx waveform. Calculations in Warp and LSP show a space charge effect at the beam head that was not measured. The calculations also reproduce the slopes measured in the Marx waveform giving slight disagreement between the calculations and measurements of the longitudinal phase space. This indicates the Marx waveform does not accurately represent the dynamics of the longitudinal beam bunch from head to tail.

\subsection{Future recommendations}

The NDCX campaign has demonstrated capabilities to begin ion based target heating experiments. However, like any experimental campaign there are still physics questions to be answered and additional experiments and calculations that can be performed. Listed below are several suggestions that I have, which the Heavy Ion Fusion Science Virtual National Laboratory (HIFS-VNL) may want to examine. Some of the suggestions are specific questions raised in my thesis and others are general recommendations.

In order to accurately measure axial compression the response time of the diag- 
nostic used must be less than the expected (based on PIC simulations) and measured pulse widths. The measurements of axial compression (Chapter 6) and longitudinal temperature (Chapter 7) in this thesis indicate we are at this threshold. The time response and after glow time of the alumina scintillator materials used for the optical Faraday cup (Section 3.1.3) have not been quantified. It is highly recommended to develop diagnostics with sub-ns resolution to measure axial compression (i.e. the fast pinhole Faraday cup and scintillator material). In Section 3.1.2 there are suggestions for improving the temporal resolution of the fast pinhole Faraday cup.

Demonstrating initial target heating to WDM is one of the near term goals for the HIFS-VNL program. In order to do this the main objective is to maximize the ion energy deposition on target $(\varepsilon)$. Listed below are additional methods or experiments that may be performed to help increase $\varepsilon$ and provide more flexibility to the NDCX. These are not listed in any order of importance.

First, there is no experimental proof that the magnetic dipole and electron trap used downstream of the induction gap are necessary to prevent backstreaming plasma electrons. These hardware add $28 \mathrm{~cm}$ of space charge dominated drift between the induction gap and the neutralization section (Chapter 6). If this hardware can be removed it will reduce envelope restrictions upstream in the beam transport lattice and space charge effects downstream of the induction gap.

Second, chromatic aberrations caused by the velocity tilt reduce $\varepsilon$. It may be beneficial to develop a time dependent focusing element to correct the chromatic aberration. There are several applications that exist an einzel lens or electrostatic quadrupole lattice can be used for low-energy applications or possibly a wiggler magnet for higher energy applications.

Third, induction acceleration and bunching are two methods in which $\varepsilon$ can be 
increased. Currently, only induction bunching is used on the NDCX. Induction acceleration is an attractive tool for increasing the beam intensity on target for the NDCX. With the induction cells available it may be useful to test the capabilities.

Finally, experiments and simulations with the final focus geometry have indicated the difficulty in filling the beam transport section with the necessary amount of plasma to yield sufficient neutralization (Section 6.5). There are several options that may improve this situation. Calculations and experiments which manipulate the magnetic field topology in the final focus region may indicate possible solutions. These can include the use of additional magnets or diamagnetic materials. In addition a lower field, larger bore solenoid may reduce plasma injection issues. Listed below are some of the advantages of using a lower field, larger bore solenoid:

1. A larger bore increases the radius of field lines and may reduce the mirroring of the plasma as it enters the solenoid.

2. Plasma sources could be installed in a larger bore solenoid reducing plasma filling issues and providing flexibility to the system.

3. A larger bore solenoid also reduces envelope and centroid restrictions which are currently pretty tight $(<1 \mathrm{~mm}$ and $<1 \mathrm{mrad}$ ), increasing the flexibility of the system for longer focal lengths.

One disadvantage to using a lower field solenoid is it may reduce the convergence angle of the beam to the target, increasing the final spot size achievable. Each of these suggestions made above will require additional research to determine their likelihood of success, but they should be considered. 


\section{Bibliography}

[1] J.D. Lawson's original report on fusion reactors (initially classified).

[2] J. D. Lawson, Proceedings of the Physical Society B, Vol. 70, p. 6 (1957).

[3] https://lasers.llnl.gov/.

[4] http://en.wikipedia.org/wiki/fusion_energy/.

[5] http://www-ssrl.slac.stanford.edu/lcls/talks/rlee_wdm_100400.pdf.

[6] http://hifweb.lbl.gov/public/AcceleratorWDM/proceedings/1.Wednesday.Feb.22/ am11.40.D.Lee.pdf.

[7] Workshop on Accelerator Driven Warm Dense Matter Physics, Four Point Sheraton Hotel, Pleasanton, CA, February 22-24, 2006.

[8] http://hifweb.lbl.gov/public/AcceleratorWDM/proceedings/1.Wednesday.Feb.22/ am09.00.R.More.pdf.

[9] P.K Roy et al., Phys. Rev. Lett. 95234801 (2005).

[10] P.A.Seidl et. al., Nucl. Instrum. Methods Phys. Res. A 577, 215 (2007).

[11] J.E. Coleman et al., in Proceedings of the Particle Accelerator Conference, Albuquerque, NM, 2007, p. 3516, http://www.JACoW.org/.

[12] J.E. Coleman et al., Phys. Rev. ST Accel. Beams 11, 050101 (2008).

[13] J.E. Coleman et al., Phys. Rev. ST Accel. Beams 11, 050103 (2008).

[14] D. Shuman et al., in Proceedings of the Particle Accelerator Conference, Knoxville, TN, 2005, p. 3798, http://www.JACoW.org/.

[15] P.C. Efthimion et al., Nucl. Instrum. Meth. Phys. Res. A 544, 387 (2005).

[16] A. Anders and G.Y. Yushkov, J. Appl. Phys. 91, 4824 (2002).

[17] Francis F. Chen, Introduction to Plasma Physics, Plenum Press, New York (1974). 
[18] Dwight R. Nicholson, Introduction to Plasma Theory, John Wiley and Sons Inc., New York, (1983).

[19] Michael A. Lieberman and Allen J. Lichtenberg, Principles of Discharges and Materials Processing, John Wiley and Sons Inc., New York, (1994).

[20] Martin Reiser, Theory and Design of Charged Particle Beams, John Wiley and Sons Inc., New York (1994).

[21] Stanley Humphries Jr., Charged Particle Beams, John Wiley and Sons Inc., New York, (2002).

[22] Helmut Wiedemann, Particle Accelerator Physics I, Second Edition, SpringerVerlag Berlin Heidelberg (2003).

[23] Francis F. Chen, Introduction to Plasma Physics, Plenum Press, New York, p. 17-19 (1974).

[24] Martin Reiser, Theory and Design of Charged Particle Beams, John Wiley and Sons Inc., New York, p. 15 (1994).

[25] Michael A. Lieberman and Allen J. Lichtenberg, Principles of Discharges and Materials Processing, John Wiley and Sons Inc., New York, p. 40-41 (1994).

[26] Michael A. Lieberman and Allen J. Lichtenberg, Principles of Discharges and Materials Processing, John Wiley and Sons Inc., New York, p. 91 (1994).

[27] Michael A. Lieberman and Allen J. Lichtenberg, Principles of Discharges and Materials Processing, John Wiley and Sons Inc., New York, p. 164-165 (1994).

[28] Dwight R. Nicholson, Introduction to Plasma Theory, John Wiley and Sons Inc., New York, p. 37 (1983).

[29] Martin Reiser, Theory and Design of Charged Particle Beams, John Wiley and Sons Inc., New York p. 62 (1994).

[30] Stanley Humphries Jr., Charged Particle Beams, John Wiley and Sons Inc., New York, p. 36 (2002).

[31] Helmut Wiedemann, Particle Accelerator Physics I, Second Edition, SpringerVerlag Berlin Heidelberg, p. 149 (2003).

[32] Martin Reiser, Theory and Design of Charged Particle Beams, John Wiley and Sons Inc., New York p. 66 (1994).

[33] Stanley Humphries Jr., Charged Particle Beams, John Wiley and Sons Inc., New York, p. 401 (2002). 
[34] Martin Reiser, Theory and Design of Charged Particle Beams, John Wiley and Sons Inc., New York, p. 210, 318 (1994).

[35] Y.-J. Chen et. al., Nucl. Instrum. Methods Phys. Res. A 292, 455 (1990).

[36] Y.-J. Chen, Nucl. Instrum. Methods Phys. Res. A 398, 139 (1997).

[37] Y.-J. Chen et. al., in Proceedings of the Particle Accelerator Conference, San Francisco, CA, 1991, p. 3100.

[38] G. Budker, G. Dimov, and V. Dudnikov, Sov. At. Energy 22, 5 (1967).

[39] O. Gröbner, in Proceedings of the 10th International Conference on High-Energy Accelerators, Protvino, Russia (Institute of High Energy Physics, Protvino, 1977), p. 277.

[40] M. Izawa, Y. Sato, and T. Toyomasu, Phys. Rev. Lett. 74, 5044 (1995).

[41] K. Ohmi, Phys. Rev. Lett. 75, 1526 (1995).

[42] W. T. Weng et al., in Proceedings of the Particle Accelerator Conference, Vancouver, Canada, 1997, p. 970, http://www.JACoW.org/.

[43] R. J. Macek et al., in Proceedings of the Particle Accelerator Conference, Chicago, IL, 2001, p. 688, http://www.JACoW.org/.

[44] M.A. Furman et al., Phys. Rev. ST Accel. Beams 5, 124404 (2002).

[45] S.Y. Zhang et al., in Proceedings of the European Particle Accelerator Conference, Lucerne, Switzerland, 2004, p. 947, http://www.JACoW.org/.

[46] J.W. Flanagan et al., Phys. Rev. Lett. 94, 054801 (2005).

[47] R.J. Macek and A. A. Browman, in Proceedings of the Particle Accelerator Conference, Knoxville, TN, 2005, p. 2547, http://www.JACoW.org/.

[48] D. Edwards, in Proceedings of the Particle Accelerator Conference, San Francisco, CA, 1979, p. 4089, http://www.JACoW.org/.

[49] P. Thieberger et al., Phys. Rev. St Accel. Beams 7093201 (2004).

[50] A.W. Molvik et al., Phys. Rev. ST Accel. Beams 7093202 (2004).

[51] E. Mahner et al., Phys. Rev. St Accel. Beams 7103202 (2004).

[52] E. Mahner et al., Phys. Rev. St Accel. Beams 8053201 (2005).

[53] H. Huang et al., in Proceedings of the European Particle Accelerator Conference, Edinburgh, Scotland, 2006, p. 583, http://www.JACoW.org/. 
[54] M. Bender et al., in Proceedings of the European Particle Accelerator Conference, Edinburgh, Scotland, 2006, p. 1423, http://www.JACoW.org/.

[55] M. Kireeff Covo et al., Phys. Rev. ST Accel. Beams 9063201 (2006).

[56] M. Kireeff Covo et al., Phys. Rev. Lett. 97054801 (2006).

[57] A. W. Molvik et al., Phys. Rev. Lett. 98064801 (2007).

[58] F.M. Bieniosek et al., Phys. Rev. St Accel. Beams 10093201 (2007).

[59] W.M. Sharp et al., Nucl. Instrum. Methods Phys. Res. A 577, 146 (2007).

[60] Martin Reiser, Theory and Design of Charged Particle Beams, John Wiley and Sons Inc., New York p. 395 (1994).

[61] Martin Reiser, Theory and Design of Charged Particle Beams, John Wiley and Sons Inc., New York p. 397 (1994).

[62] J.J. Barnard and S.M. Lund Beam Physics with Intense Space Charge, Course notes of the United States Particle Accelerator School held June 12-23, 2006 in Boston, Massachusetts.

[63] Martin Reiser, Theory and Design of Charged Particle Beams, John Wiley and Sons Inc., New York p. 504-505 (1994).

[64] R.J. Briggs et al., Phys. Rev. ST Accel. Beams 9060401 (2006).

[65] P.K. Roy et al., Phys. Rev. ST Accel. Beams 9070402 (2006).

[66] J. E. Coleman et al., Nucl. Instrum. Methods Phys. Res. A 577, 197 (2007).

[67] D. D.-M. Ho et al., Particle Accelerators 35, 15 (1991).

[68] H. Qin et al., Phys. Rev. ST Accel. Beams 7, 104201 (2004).

[69] W. M. Sharp et al., Nucl. Instrum. Meth. Phys. Res. A 544, 398 (2005).

[70] W. M. Fawley et al., Phys. Plasmas 4, 880 (1997).

[71] D.R. Welch et al., Nucl. Instrum. Methods Phys. Res. A 577, 231 (2007).

[72] A.B. Sefkow et al., Phys. Rev. ST Accel. Beams 9, 052801 (2006).

[73] http://www.valleydesign.com/.

[74] http://www.industrialnetting.com/metal_mesh.htm.

[75] http://jp.hamamatsu.com/products/sensor-etd/pd004/index_en.html.

[76] http://www.displaytech.com/photonics_shutters.html. 
[77] F.M. Bieniosek et. al., Nucl. Instrum. Methods Phys. Res. A 544, 268 (2005).

[78] http://www.piacton.com/products/imcam/pimax/default.aspx

[79] R.E. Warren et. al., Rev. Sci Instrum. 18, 559 (1947).

[80] http://sales.hamamatsu.com/en/products/system-division/ultra-fast/streaksystems/part-c7700.php.

[81] http://www.pearsonelectronics.com/

[82] http://www.tek.com/products/oscilloscopes.

[83] http://www.ni.com/labview/.

[84] L.R. Prost, "Parametric study of the current limit within a single driver-scale transport beam line of an induction Linac for Heavy Ion Fusion," Ph.D. thesis, University of California, Berkeley, 2004.

[85] http://www.piacton.com/products/software.aspx.

[86] http://rsb.info.nih.gov/ij/.

[87] http://www.mathworks.com/.

[88] M. J. L. De Hoon, W. Fawley, and J. Novotny, Java Envelope: A code for beam envelope modeling, computer program written in the Java programming language, based on the Basic code "Rematch" written by K. Hahn.

[89] Stephen C. Chapra and Raymond P. Camale, Numerical Methods for Engineers, Third Edition, McGraw-Hill Companies, Inc., Boston p.701-702 (1998).

[90] http://www.ptc.com/appserver/mkt/products/home.jsp?k=3901.

[91] A. Friedman et al., Phys. Fluids B 4, 2203 (1992).

[92] D.P. Grote et al., AIP Conf. Proc. 391, 51 (1996).

[93] D.P. Grote et al., Nucl. Instrum. Methods Phys. Res. A 415, 428 (1998).

[94] D. P. Grote et al., AIP Conf. Proc. 749, 55 (2005).

[95] http://www.python.org/.

[96] J.-L. Vay et al., Nucl. Instrum. Meth. Phys. Res. A 577, 65 (2007).

[97] See /http://www.txcorp.com/technologies/TxPhysics/S.

[98] J.-L. Vay, et al., in Proceedings of the Particle Accelerator Conference, Knoxville, TN, 2005, p. 1479 http://www.jacow.org/. 
[99] R.H. Cohen, et al., Phys. Plasmas 12, 056708 (2005).

[100] J.-L. Vay, et al., Phys. Plasmas 11 5, 2928 (2004).

[101] LSP is a software product of ATK Mission Research, http://www.mrcwdc.com/LSP/index.html.

[102] D. R. Welch et al., Nucl. Instrum. Methods Phys. Res. A 464, 134 (2001).

[103] D.R. Welch et al., Comp. Phys. Comm. 164, 183 (2004).

[104] D.R. Welch et al., "Source-to-target simulation of simultaneous longitudinal and transverse focusing of heavy ion beams," to be published in Phys. Rev. ST Accel. Beams (2008).

[105] C.F. Chan et. al., Nucl. Instrum. Methods Phys. Res. A 415, 274 (1998).

[106] E. Lee, Nucl. Instrum. Methods Phys. Res. A 544, 187 (2005).

[107] H.V. Smith et. al., in Proceedings of the Particle Accelerator Conference, New York, NY, 1999, p. 1929.

[108] A. Ueno et. al., in Proceedings of the XX International Linac Conference, Monterey, CA, 1999, p. 256.

[109] A. Jakob et al., in Proceedings of the European Particle Accelerator Conference, Paris, France, 2002, p. 1906, http://www.JACoW.org/.

[110] E. Fagotti et. al., in Proceedings of the 2004 International Linac Conference, Lübeck, Germany, 2004, p. 72.

[111] J.P. Blewett and E.J. Jones, Phys. Rev. 50, 464 (1936).

[112] A.I. Warwick in Proceedings of the Particle Accelerator Conference, Vancouver, Canada, 1985, p. 1809, http://www.JACoW.org/.

[113] J. Armijo, LBNL Report No. 61975, 2006.

[114] W. Jiang and Magne Kristiansen, Phys. Plasmas 8, 3781 (2001).

[115] S. Eylon et al., in Proceedings of the Particle Accelerator Conference, Portland, OR, 2003, p. 2616, http://www.JACoW.org/.

[116] Y.-J. Chen et. al., in Proceedings of the XIX International Linac Conference, Chicago, IL, 1998, p. 469.

[117] T.L. Houck et. al., in Proceedings of the XIX International Linac Conference, Chicago, IL, 1998, p. 857. 
[118] S.M. Lund et al., "Correction of misalignment induced transverse centroid oscillations in solenoidially focused beam transport lattices," to be submitted to Phys. Rev. ST Accel. Beams (2008).

[119] G. Rosenman, et al., Appl. Phys. Rev. 88, 6109 (2000).

[120] A. Dunaevsky, et al., JAP 90, 3689 (2001).

[121] A. Dunaevsky, et al., JAP 95, 4621 (2004).

[122] J.J. Barnard et al., in Proceedings of the Particle Accelerator Conference, Knoxville, Tennessee, 2005, p. 2568, http://www.JACoW.org/.

[123] E.P. Gilson, private communication.

[124] A. Anders et al., Rev. Sci Instrum. 70, 4532 (1999).

[125] A.B. Sefkow, "Current density compression of intense ion beams," Ph.D. thesis, Princeton University, 2007.

[126] Acree Technologies Incorporated, private communication.

[127] M. Kireeff-Covo and I. Kaganovich, private communication.

[128] P.K. Roy et al., in Proceedings of the Particle Accelerator Conference, Albuquerque, NM, 2007, p. 3519, http://www.JACoW.org/.

[129] P.K. Roy, private communication.

[130] F.M. Bieniosek et al., Nucl. Instrum. Meth. Phys. Res. A 577, 284 (2007).

[131] E. Henestroza et al., Phys. Rev. ST Accel. Beams 8, 052801 (2005). 